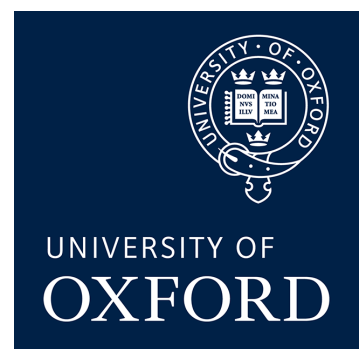

DePARTMENT OF PHYSICS

$2016-2020$

\title{
SEARCH FOR ELECTRON NEUTRINO ANOMALIES WITH THE MICROBOONE DETECTOR
}

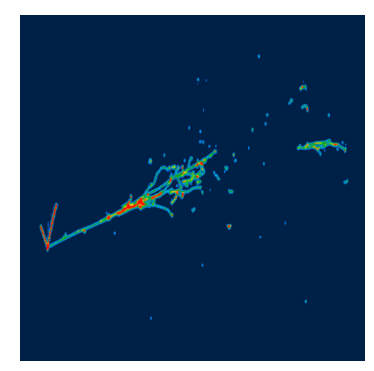

\author{
Wouter Van De Pontseele \\ Jesus College, University of Oxford
}

Supervisors: Prof Roxanne Guenette Prof Alfons Weber

A thesis submitted for the degree of Doctor of Philosophy 



\section{Statement of Originality}

This thesis and the work presented in it are my own and were produced by me as a result of my own original research. Results and figures from published works by others have been clearly attributed and the assistance received in preparing this thesis has been acknowledged. Furthermore, this work has not been submitted for another qualification at this or any other university.

Chapter 1 contains a brief introduction, Chapter 2 gives some background information about the theory motivating the work presented in this thesis, and Chapter 3 describes the MicroBooNE experiment whose data was analysed in this work. These chapters represent my summary of the work of others, and all relevant papers and documents have been cited. Chapter 4 describes the reconstruction techniques used to allow a high-level analysis of the data. The different components of this thesis are described in Chapters 5, 6, 7, 8 and 9, and, where not specified otherwise, is entirely my own work. 


\section{Acknowledgements}

It is not an understatement to say that without my supervisor, Roxanne, this journey - let alone this thesis - would never have been completed. I owe you an enormous thank you for enabling and supporting me to pursue the research I could not have imagined to be part of before commencing my DPhil. Your guidance and ideas are present everywhere in this document, not at least through the thousands of comments you supplied me with. Thank you even more for the moose burger BBQ and Christmas parties you hosted, back in the days that corona was not a thing yet.

I want to thank all the scientists involved in the design, construction and commissioning of MicroBooNE; enabling me to act as if neutrino data grows on the trees. A big thank you to all organisations that financially and logistically supported my DPhil: the University of Oxford, Harvard University, Jesus College, Oxford University Press and Fermilab. Thank you, Sue and Kim, for the complicated arrangements I often needed your help with.

Santiago, thank for your introduction in authentic Latin-American fiesta music and your imitation of the pelican. Peter, thank you for visiting me in Boston on my birthday without realising by surprise. And Priyav, thank you for always going on trips and adventures, even when it involves landing a glider without reading the safety instructions. Luca, thank you for attending the most obscure Clarendon events. Miha, thank you for smuggling me into Vincent's Club, and even lending me your tie. Ben, thank you for joining the expeditions to Zuzu's kitchen. All other Belgians in Boston, thank you for convincing me that there is always the option for a second beer. Supraja, Avinay, Krishan and Raquel, thanks for making my time at Fermilab wonderful and joining me on haunted wine walks at the local hairdresser. Andy S. \& Lorena, you were amazingly helpful whenever pandora problems occurred, thank you for that. Lars, thank you for making sure there was never shortage of free food supplies at Harvard, even non-pizza lunches. Roberto, thank you for sledging down a mountain with me. I hope the Korean girl is okay by now. Nicolò, thanks for all our conversations, but especially the ones while eating frozen thanksgiving lunch in the snow in the middle of nowhere. And, of course, Marco, thank you for being my inflatable boat companion during fireworks on the river.

A huge thank you to my family: mam, dad and Lisa, for always coping with me and making Belgium still feel like my home. Finally, Aurélie, I dedicate this thesis to you, in the hope you will feel a little obliged to read something slightly scientific when you grow older. 


\section{Abstract}

The Micro Booster Neutrino Experiment (MicroBooNE) is a Liquid Argon Time Projection Chamber (LArTPC) located in the Booster Neutrino Beam at Fermi National Accelerator Laboratory. The experiment was devised to investigate a series of observed anomalies concerning short-baseline neutrino oscillation physics. The LArTPC technology enables the experiment to study neutrino-argon scattering with an unprecedented detail.

This thesis presents a cosmic-ray characterisation and rate measurement. The understanding of cosmic activity in the detector - MicroBooNE's dominant background - is then used to develop cosmic rejection tools. A flavour-agnostic neutrino selection is constructed, which forms the cornerstone of this and further analyses. Inclusive muon and electron charged-current neutrino interaction selections with unprecedented purity and efficiency are presented.

The first fully-automated characterisation of electron neutrinos in a muon neutrino beam with the LArTPC detector technology is performed. The Booster Neutrino Beam has an energy peaking around $1 \mathrm{GeV}$ and an intrinsic electron content of approximately $0.5 \%$. The analysis investigates electrons produced in charged-current electron neutrino interactions. The kinematics of the electrons are measured along with comparisons to simulation. Most of the systematic uncertainties are constrained using a data-driven sample of chargedcurrent muon neutrino events. The measurement of electron neutrinos originating from the Booster Neutrino Beam is a crucial component towards understanding the nature of the observed excess of low-energy electromagnetic-like events at its predecessor, MiniBooNE. 


\section{Contents}

Abstract vii

1 Introduction 1

2 Neutrino Physics 5

2.1 Neutrinos beyond the Standard Model . . . . . . . . . . . . . . . . . . . 5

2.2 Neutrino oscillations . . . . . . . . . . . . . . . . . . . . . . . . 11

2.3 The sterile neutrino hypothesis . . . . . . . . . . . . . . . 15

2.3.1 The simplest $3+1$ extension . . . . . . . . . . . . . . . . . . . 15

2.4 Short-baseline anomalies . . . . . . . . . . . . . . . . . . . 18

2.4.1 Accelerator neutrino experiments . . . . . . . . . . . . . . 18

2.4.2 Radio-chemical neutrino detection experiments . . . . . . . . . . . . 24

2.4.3 Reactor Antineutrino Anomaly (RAA) . . . . . . . . . . . . . . . . . 24

2.4 .4 Current status \& global fits . . . . . . . . . . . . . . . . . . . . 25

2.5 Neutrino interactions . . . . . . . . . . . . . . . . . . . . 28

2.5 .1 Nuclear effects . . . . . . . . . . . . . . . . . . . . . 28

2.5 .2 Neutrino interaction modes . . . . . . . . . . . . . . . 30

2.5.3 Neutrino interaction modelling in MicroBooNE . . . . . . . . . . . 32

3 The MicroBooNE Experiment 35

3.1 Neutrino beams at Fermilab . . . . . . . . . . . . . . . . . . . 36

3.1.1 Booster Neutrino Beam (BNB) f . . . . . . . . . . . . . . 37

3.1 .2 Neutrinos from the Main Injector (NuMI) . . . . . . . . . . . . . . . 39

3.2 The MicroBooNE detector . . . . . . . . . . . . . . . . . . . . . . . . 41

3.2.1 The LArTPC technology . . . . . . . . . . . . . . . . . . . . . . 43

3.2.2 Light production \& collection in MicroBooNE . . . . . . . . . . . . . 46

3.2.3 The cosmic ray tagger system . . . . . . . . . . . . . . . . . . 51

3.3 Physics motivations . . . . . . . . . . . . . . . . . 53

3.3.1 Validation of short-baseline anomalies . . . . . . . . . . . . . . 53

3.3.2 Cross section measurements \& study of nuclear effects . . . . . . . . 53

3.3.3 Astro-particle physics \& exotic physics . . . . . . . . . . . . . 54

3.4 Beyond MicroBooNE: the Short-Baseline Neutrino Programme . . . . . . . . . 56

4 Triggering \& Event Reconstruction 59

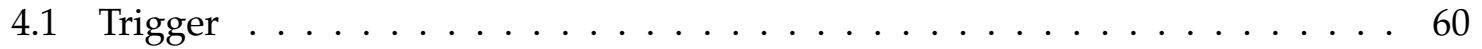

4.1 .1 Hardware triggering . . . . . . . . . . . . . . . . . . 60

4.1 .2 Software triggering . . . . . . . . . . . . . . 61

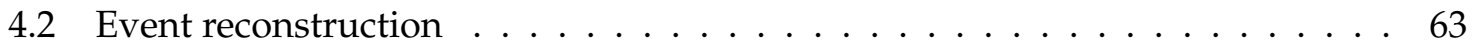


4.2.1 Optical reconstruction . . . . . . . . . . . . . . . . . 63

4.2 .2 TPC signal processing . . . . . . . . . . . . . . . . . . . . 64

4.2.3 Pandora reconstruction framework . . . . . . . . . . . . 66

4.2 .4 Event simulation . . . . . . . . . . . . . . . . . 70

5 Cosmic-Ray Measurements $\quad 73$

5.1 The cosmogenic flux simulation . . . . . . . . . . . . . . . . . . . . . . 74

5.1 .1 Geometry . . . . . . . . . . . . . . . . . 74

5.1 .2 Cosmic-ray generators . . . . . . . . . . . . . . . . 75

5.1 .3 Simulated muon rate . . . . . . . . . . . . . . . . . . 77

5.2 TPC-based muon rate \& reconstruction resolution . . . . . . . . . . . . . 81

5.2.1 Reconstruction purity and efficiency . . . . . . . . . . . . . . . 81

5.2.2 Data and Monte Carlo comparisons for the muon rate . . . . . . . . . 82

5.2.3 Reconstruction muon resolution and data/MC distributions . . . . . . 83

5.2 .4 Systematic error estimation . . . . . . . . . . . . . . . . . . 90

5.3 PMT-based muon rate measurement . . . . . . . . . . . . . . . . . . 92

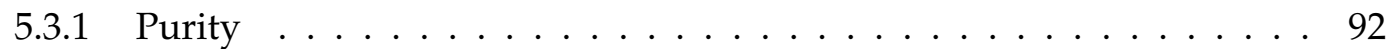

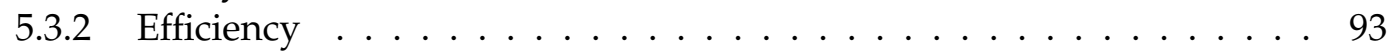

5.3.3 Rate comparison in data and Monte Carlo . . . . . . . . . . . . . . 95

5.3 .4 Systematic error estimation . . . . . . . . . . . . . . . . 95

5.4 Towards a CRT-based muon rate measurement . . . . . . . . . . . . . . . 98

5.5 Results \& discussion . . . . . . . . . . . . . . . . . . . . . . . . . 101

6 A Flavour Agnostic Neutrino Selection $\quad 105$

6.1 Signal definition \& normalisation . . . . . . . . . . . . . . . . . 106

6.2 Optical selection . . . . . . . . . . . . . . . . . . . . . . . . . . . . 109

6.2 .1 Flash selection . . . . . . . . . . . . . . . . . . . . . 110

6.2.2 Flash compatibility with neutrino candidates . . . . . . . . . . . 111

6.3 Neutrino candidate selection . . . . . . . . . . . . . . . . . . 122

6.3.1 Topological Support Vector Machine . . . . . . . . . . . . . . . . . . . 122

6.3 .2 Flash-matching . . . . . . . . . . . . . . . . . . . . . . 124

6.3 .3 Obvious cosmic flash-matching . . . . . . . . . . . . . . . 127

6.4 NeutrinoID performance . . . . . . . . . . . . . . . . . . . . . . . . . . 129

6.5 Additional cosmic rejection using the CRT . . . . . . . . . . . . . . . . . . 137

7 Charged-Current Muon Neutrino Selection 141

7.1 Motivation . . . . . . . . . . . . . . . . . . . . . . . . . . . 142

7.1.1 Inclusive charged-current muon neutrino selection . . . . . . . . . . 142

7.1.2 Contained high-purity $v_{\mu}$ CC selection . . . . . . . . . . . 143

7.1 .3 Selection strategy . . . . . . . . . . . . . . . . . . . . 144

7.2 Event preselection . . . . . . . . . . . . . . . . . . . . . . 145

7.3 Muon Identification . . . . . . . . . . . . . . . . . . . . . . . 148

7.3.1 Proton-muon separation using the likelihood ratio . . . . . . . . . . 148

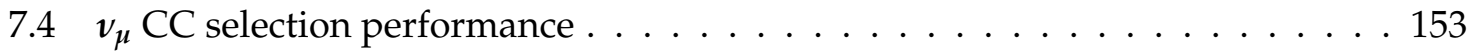

7.4 .1 Inclusive selection . . . . . . . . . . . . . . . . . . . 153

7.4 .2 Contained selection . . . . . . . . . . . . . . . . . 155

7.4 .3 Exclusive $v_{\mu}$ CC selections . . . . . . . . . . . . . . . 161 
8 Charged-Current Electron Neutrino Selection 163

8.1 Motivation . . . . . . . . . . . . . . . . . . . . . . . . 164

8.1.1 Electron neutrino selection philosophy . . . . . . . . . . . . . . 164

8.1 .2 Selection strategy . . . . . . . . . . . . . . . . . . . 165

8.1.3 MiniBooNE LEE signal model . . . . . . . . . . . . . . . . 166

$8.2 v_{e}$ CC preselection . . . . . . . . . . . . . . . . . . . 169

8.3 Electron Identification $\ldots \ldots \ldots \ldots$

8.3 .1 Electromagnetic shower formation . . . . . . . . . . . . . . . 173

8.3 .2 Shower variables . . . . . . . . . . . . . . . . . 176

8.3.3 Classification using a boosted decision tree . . . . . . . . . . . . . . 179

8.3.4 Boosted decision tree performance . . . . . . . . . . . . . . . . . . 182

8.4 Muon neutrino induced backgrounds . . . . . . . . . . . . . . . . . . 188

8.5 Inclusive $v_{e}$ CC selection . . . . . . . . . . . . . . . . . . . . . 192

8.5.1 Purity, efficiency \& remaining backgrounds . . . . . . . . . . . . . . . . 193

8.5.2 Limitations of the reconstruction framework . . . . . . . . . . . . . . 194

9 Data-Driven $v_{\mu}$ Constraint \& Charged-Current $v_{e}$ Measurement with the BNB 201

9.1 Sources \& treatment of systematic uncertainties . . . . . . . . . . . . . . . 202

9.1.1 BNB flux prediction . . . . . . . . . . . . . . . . . . 205

9.1 .2 Cross-section modelling . . . . . . . . . . . . . . . . 205

9.1 .3 Detector systematic uncertainties . . . . . . . . . . . . . . . . 206

9.2 Constraining the systematic uncertainties . . . . . . . . . . . . . . . . . . 212

9.3 Electron neutrino measurement \& sensitivity to new physics . . . . . . . . . 218

9.3.1 Statistical-only likelihood construction and sensitivity estimation . . . 218

9.3.2 The intrinsic $v_{e}$ BNB component . . . . . . . . . . . . . . . . 222

9.3.3 Sensitivity to a $3+1 v_{S}$ oscillation signal . . . . . . . . . . . . 223

9.3.4 Sensitivity to the MiniBooNE low-energy excess . . . . . . . . . . . 225

10 Conclusion \& Outlook $\quad 227$

A Unresponsive Wires \& Charge Calibration $\quad 231$

B The Space-Charge Effect $\quad 235$

C Data-sets \& Energy Threshold Determination in the Cosmic-ray Measurement 239

C.1 Samples . . . . . . . . . . . . . . . . . . . . . . . 239

C.2 Normalisation . . . . . . . . . . . . . . . . . . . . . . . . . . 240

C.3 Energy threshold and dependence . . . . . . . . . . . . . . . . . . . 242

D Flux \& Cross-Section Variations $\quad 245$

$\begin{array}{lr}\text { Acronyms } & 249\end{array}$

$\begin{array}{ll}\text { List of Figures } & 253\end{array}$

$\begin{array}{lr}\text { List of Tables } & 259\end{array}$ 
The White Rabbit put on his spectacles. "Where shall I begin, please your Majesty?" he asked.

"Begin at the beginning," the King said gravely, "and go on till you come to the end; then stop."

\section{Introduction}

The road to new physics is often paved with interesting anomalies. This is especially true if one looks at the history of neutrino physics. One example is the solar neutrino puzzle. The apparent disappearance of neutrinos from the sun was observed in the mid-1960s and finally resolved around 2002. It took 40 years to conclusively prove that neutrinos oscillate. Now - almost two decades later - the neutrino standard model is faced with a new set of anomalies. These anomalies are referred to as the short-baseline anomalies.

In Chapter 2, a brief recap of neutrino physics is given. Furthermore, neutrino oscillations are explained and a simple extension with a hypothetical sterile neutrino is introduced. A series of experiments that took place over the last 25 years are used to illustrate the current status of the field, and especially the short-baseline anomalies with particular attention given to the MiniBooNE experiment. In 2018, MiniBooNE confirmed its earlier observation 
of an excess of low-energy electromagnetic events with additional data and constrained uncertainties.

MicroBooNE, equipped with a modern detector technology - the Liquid Argon Time Projection Chamber - was designed to address the MiniBooNE anomalous result. In Chapter 3, the characteristics of the MicroBooNE experiment, and of the neutrino beams, are introduced.

The novel combination of the technology with a high-intensity neutrino beam, a large fiducial volume and surface operation give rise to challenges in the event reconstruction. The different steps in the scintillation light-based event trigger and automatic pattern reconstruction chain are the topic of Chapter 4.

The largest fraction of recorded MicroBooNE data consists of cosmic activity. Chapter 5 studies the cosmic-muon rate with the three different sub-detector systems that were introduced in Chapter 3.

Chapter 6 exploits the knowledge of the cosmic background to develop a set of tools to reject cosmic-related activity and isolate neutrino events in the time projection chamber. This neutrino selection is agnostic with respect to the neutrino flavour and forms the foundation of several neutrino selections targeting a wide variety of physics in MicroBooNE.

One example of such a selection is introduced in Chapter 7. There, the flavour-agnostic neutrino selection is combined with particle identification tools to obtain a sample of pure charged-current interactions. It will be demonstrated that this selection gives access to a set of exclusive channels that relates to different neutrino interaction modes.

The final portion of this thesis fully focuses on electron neutrinos. In Chapter 8, electromagnetic shower formation in liquid argon is studied. Variables are constructed to distinguish electron and photon showers. Machine-learning algorithms are used to perform particle identification and event classification.

Chapter 9 builds on the event sample of $v_{e}$ CC interactions obtained in Chapter 8. The 
sources of systematic uncertainties are discussed and their impact on the electron kinematics is evaluated. A data-driven method to constrain the uncertainties by using $v_{\mu} \mathrm{CC}$ events is introduced. Finally, the sensitivity of the selection to anomalies in the expected electron neutrino rate is studied. 


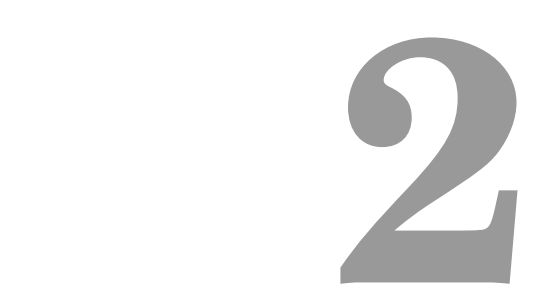

Neutrino Physics

\subsection{Neutrinos beyond the Standard Model}

Through decades of work, physicists have refined a model to describe the particles that are considered to be fundamental, and their interactions. This masterpiece of science is called the Standard Model of particle physics. While this theory is incredibly accurate, we know of at least one particle that exhibits behaviours that are pushing the boundaries of its scope. These particles are called neutrinos, and because they interact only via the weak nuclear force, they can travel enormous distances through matter without being stopped. Notwithstanding that they are very difficult to observe, great progress in the field has been achieved during the last twenty years. The study of neutrinos is opening a path for the generalisation of the Standard Model of particle physics. This framework, developed in the 
mid 1970s, consists of fundamental particles that are either the building blocks of matter, called fermions, or the mediators of interactions, called bosons (Figure 2.1).

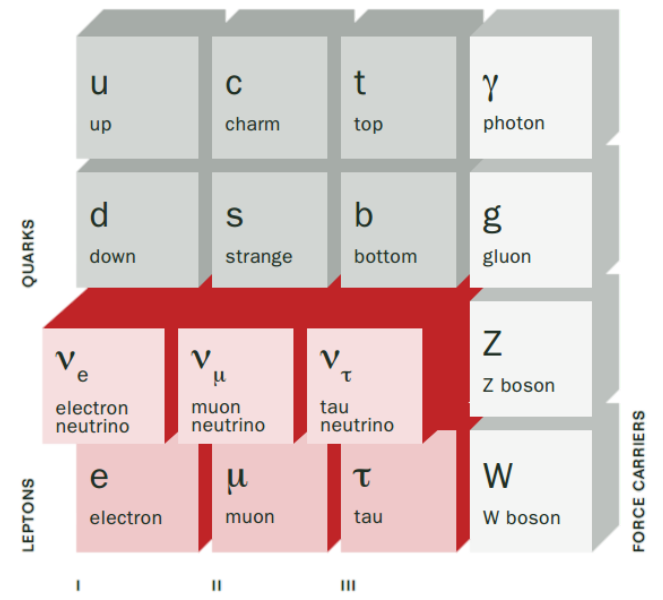

Figure 2.1: The Standard Model of particle physics, with the three generations of fermions on the left and the gauge bosons in the fourth column. Figure from [1].

The electroweak theory, the precursor of the Standard Model, developed in late 1960s, incorporates neutrinos $\left(v_{e}, v_{\mu}, v_{\tau}\right)$ as left-handed partners of the three families of charged leptons $\left(e^{-}, \mu^{-}, \tau^{-}\right)$, which is illustrated in Figure 2.2. Neutrinos were assumed to be massless and their individual lepton number was thought to be strictly conserved. Right-handed components of the neutrino field are absent in the Standard Model because, if neutrinos are massless, only their left-handed component interacts via the weak force. These assumptions are supported by the absence of observation of decays that would violate the conservation of individual lepton number. An example of such a process is the muon- and electron-number violating decay $\mu^{ \pm} \rightarrow e^{ \pm}+\gamma$. Despite the searches for it, only a stringent upper limit on the branching ratio of this decay was obtained [2].

The discovery of neutrino oscillations (Section 2.2) over the past decades provided strong evidence that the conservation of individual lepton number is incorrect. The phenomenon of neutrino oscillation is explained most easily if neutrinos are relativistic and have a small but non-zero mass. Now that neutrinos do appear to have mass, theorists have to solve two problems. The first is to overcome the contradiction between left-handedness and mass. 
Left-handed neutrino

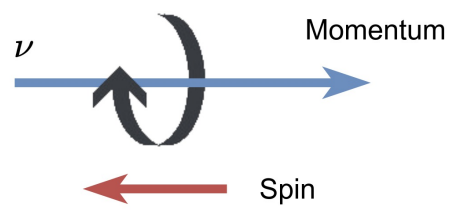

Right-handed antineutrino

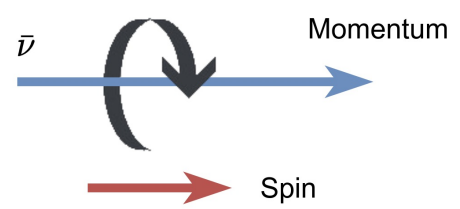

Figure 2.2: In the Standard Model of particle physics, the relative orientations of spin and linear momentum for neutrinos and antineutrinos are apparently fixed and intrinsic to the particles. For neutrinos the spin is always opposite the linear momentum and this is referred to as "left-handed", whereas the antineutrinos are always "right-handed".

The left-handed versus right-handed helicity is not exactly conserved if neutrinos have mass. A neutrino with a finite mass could spin to the left while travelling to the right and therefore be classified as left-handed. But from the reference frame of someone travelling faster than the neutrino, its velocity would be to the left, while its spin would be unchanged. This would mean that the neutrino is a right-handed particle with respect to that reference frame. Massless neutrinos, however, are travelling at the speed of light. Therefore, a reference frame to change their direction - and thereby change their helicity - does not exist.

The second is to understand why the neutrino mass is so small compared with other particle masses - the direct mass measurement recently obtained by KATRIN indicates that electrons are at least 500000 times more massive than neutrinos [3]. When neutrinos were thought to be massless, no additional terms in the Standard Model Lagrangian were needed. The tiny mass is puzzle and often requires fine-tuning of the additional terms. Instead, most theorists think that there must be a fundamental reason why the neutrino mass has a different scale.

Essentially, there are two ways to adapt the Standard Model in order to accommodate a finite neutrino mass. One approach involves new particles, called Dirac neutrinos, while the other approach involves a different type of particle, called Majorana neutrinos [4].

The idea of the Dirac neutrino works in the sense that we can generate neutrino masses via the Higgs mechanism. According to the Higgs mechanism in the Standard Model, particles 
in vacuum acquire mass as they couple to the Higgs field. Photons are massless because they do not interact with the Higgs boson. All particles that do interact with the Higgs boson change helicity when they collide with the Higgs field; left-handed particles become right-handed and vice versa. Translated to the mathematical formalism of the Standard Model; after spontaneously symmetry breaking, the Higgs doublet in the unitary gauge is:

$$
\phi(x)=\left(\begin{array}{c}
0 \\
v+h(x)
\end{array}\right),
$$

where $v=246 \mathrm{GeV}$ is the non-zero vacuum expectation value and the Higgs field $h(x)$ a perturbation. The gauge invariant interaction term of the electron field with the Higgs doublet in the Lagrangian becomes:

$$
\mathcal{L}_{e}=-m_{e}\left(\bar{e}_{L} e_{R}+\bar{e}_{R} e_{L}\right)-\frac{m_{e}}{v} h\left(\bar{e}_{L} e_{R}+\bar{e}_{R} e_{L}\right)
$$

Here, the first term is the electron mass term, coupling the left-handed positron spinor $\bar{e}_{L}$ with the right-handed electron spinor $e_{R}$ and vice-versa. The second term represents the coupling to the Higgs field $h$, with the vacuum expectation value $v$. Both terms are shown in Figure 2.3. Note that while the first term on itself, the electron mass term, is not gauge invariant under the $S U(2)_{L} \times U(1)$ electroweak unification, the combination of both terms is.
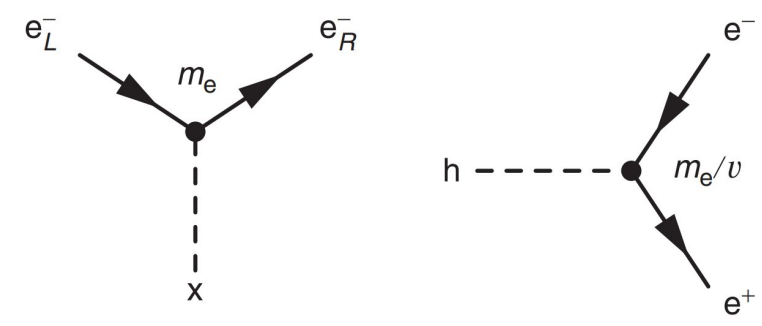

Figure 2.3: Feynman diagrams corresponding to the two terms in Equation (2.2). (Left) The interaction between an electron with left-handed chirality and the non-zero vacuum expectation value. (Right) The interaction vertex for the coupling of the Higgs perturbation to an electron. Figure from [1]. 
Experiments have shown that interacting neutrinos are left-handed [5]. If right-handed neutrinos do not exist in the Standard Model, to gauge invariant mass term can be generated by interaction with the Higgs doublet. One possible extension of the Standard Model, therefore, adds right-handed neutrinos to enable a Dirac-like mass term for neutrinos. These Dirac neutrinos acquire mass via the Higgs mechanism, but a right-handed neutrino must carry no electroweak charge, interacting only through the mass mixing with the left handed neutrino.

If neutrinos are Dirac fermions and acquire a mass proportional to their coupling to the Higgs field, they are expected to have similar masses to the other fermions in the Standard Model. Nevertheless, the coupling strength of neutrino interactions with the Higgs boson can be fine-tuned to generate masses that are compatible with experiments. This gives rise to a value that is at least twelve orders of magnitude weaker than that of the top quark.

An alternative extension to the standard Model would that the neutrino is a Majorana particle. This approach avoids the prediction of right-handed neutrinos with extremely weak interactions. However, it gives up the fundamental distinction between matter and antimatter for neutrinos. This distinction might not be required since neutrinos and antineutrinos carry no charge and behave similarly the Standard Model interactions.

Massive neutrinos sit naturally within this framework. Recall the observer travelling almost at the speed of light who overtakes a left-handed neutrino and sees a right-handed neutrino. Earlier we argued that the absence of right-handed neutrinos could mean that neutrinos are massless. But if neutrinos and antineutrinos are the same particle, then one can argue that the observer really sees a right-handed antineutrino and that the massive-neutrino hypothesis is therefore sound. In this scheme, it is possible for right-handed neutrinos to have a mass of their own without relying on additional undetected degrees of freedom in the neutrino sector.

According to the simplest Majorana-like extension of the Standard Model, type 1 seesaw, when a left-handed neutrino interacts with the Higgs boson, an extremely heavy right- 
handed neutrino is created for a brief moment before it interacts with the Higgs field to produce a light left-handed Majorana neutrino [6].

Both extensions could give rise to extra neutrino-like particles with different mass scales, some possible more advanced mechanisms to generate neutrino masses related to the two extensions discussed here, are described in [7], but are beyond the scope of this work. 


\subsection{Neutrino oscillations}

Neutrinos are produced with a specific flavour: $v_{e}, v_{\mu}$ or $v_{\tau}$. In nuclear $\beta$-decay, for example, electron antineutrinos are always produced together with an electron $\left(n \rightarrow p+e^{-}+\bar{v}_{e}\right)$. Similarly, if an anti-muon is produced in the decay of a positive pion, a muon neutrino is always produced as well $\left(\pi^{+} \rightarrow \mu^{+}+v_{\mu}\right)$. If neutrinos have a non-zero mass, and their mass eigenstates are a non-trivial mixture of their flavour eigenstates, the flavour composition will vary as a function of the distance and energy. This behaviour is called neutrino oscillation and is a consequence of the discovery that a neutrino of a given flavour is not a state of definitive mass but a superposition of several mass eigenstates. Here, this phenomenon will be explained in the case of only two mass states $v_{i}$ with different masses $m_{i}$ for $i \in\{1,2\}$. To simplify the derivation for this context, neutrinos will be treated as plane waves. More correctly is a treatment of the particles as localised wave-packets. It can be shown that even the more rudimentary plane-wave approach -which is given here - leads to the same correct answer [8].

The propagation of a quantum mechanical plane wave state $\psi$ with momentum $p$ is governed by:

$$
\psi(L)=\psi(0) e^{i p L}
$$

In the highly relativistic regime the momentum can be approximated as: $p=\sqrt{E^{2}-m^{2}} \sim$ $E-m^{2} / 2 E$, where $E, m$ are the energy and the rest mass of the particle respectively. If a neutrino propagates in vacuum over a distance $L$, it acquires a phase shift:

$$
v_{i}(L)=v_{i}(0) e^{-m_{i}^{2} L / 2 E},
$$

where the global phase is omitted. It should be noted that these expressions are only valid for plane waves in vacuum. Additional phases are needed when neutrinos propagate in a highly dense material. This coherent interaction with the medium is the MikheyevSmirnov-Wolfenstein (MSW) [9] and is discussed briefly at the end of this chapter. Now, define the two flavour states $\left(v_{e}\right.$ and $\left.v_{\mu}\right)$ as orthogonal superpositions of the definite mass 
states $\left(v_{1}\right.$ and $\left.v_{2}\right)$ :

$$
\begin{aligned}
& v_{e}=\cos \theta v_{1}+\sin \theta v_{2} \\
& v_{\mu}=-\sin \theta v_{1}+\cos \theta v_{2},
\end{aligned}
$$

in which $\theta$ is the mixing angle. Consider now a beam of electron neutrinos at $L=0$. Then

$$
v_{e}(L)=\cos \theta e^{-i m_{1}^{2} L / 2 E} v_{1}(0)+\sin \theta e^{-i m_{2}^{2} L / 2 E} v_{2}(0) .
$$

For observation purposes via the weak interaction, the mass states need to be projected back to the flavour basis:

$$
\begin{aligned}
\nu_{e}(L)=[ & \left.\cos ^{2} \theta e^{-i m_{1}^{2} L / 2 E}+\sin ^{2} \theta e^{-i m_{2}^{2} L / 2 E}\right] v_{e}(0) \\
& -\sin \theta \cos \theta\left[e^{i m_{1}^{2} L / 2 E}-e^{-i m_{2}^{2} L / 2 E}\right] v_{\mu}(0) .
\end{aligned}
$$

The probability of detecting a certain flavour is equal to the modulus square of the corresponding amplitude:

$$
\begin{aligned}
& P_{v_{e} \rightarrow v_{e}}=1-\sin ^{2} 2 \theta \sin ^{2}\left(\frac{\Delta m^{2} L}{4 E}\right) \\
& P_{v_{e} \rightarrow v_{\mu}}=\sin ^{2} 2 \theta \sin ^{2}\left(\frac{\Delta m^{2} L}{4 E}\right)
\end{aligned}
$$

where $\Delta m^{2}=m_{2}^{2}-m_{1}^{2}$ is the difference of the squares of the neutrino masses. We see that if both $\theta \neq 0$ or $\frac{\pi}{2}$ and $\Delta m^{2} \neq 0$, the composition of our neutrino beam changes in function of $L / E$. The amplitude of this oscillation is $\sin ^{2} 2 \theta$ and the wavelength in frequently used units is:

$$
L_{o s c}[\mathrm{~m}]=4 \pi \frac{E}{\Delta m^{2}}=2.48 \frac{E[\mathrm{MeV}]}{\Delta m^{2}\left[\mathrm{eV}^{2}\right]}
$$

The observation of neutrino oscillations proves that the components of the initial state indeed undergo a phase shift. As shown above, the explanation for this shift is that at least some neutrinos have mass and that the transformation between the mass and flavour eigenstates differs from the identity transformation. 
One can further generalise the neutrino mixing up to $N$ neutrino eigenstates. The probability of finding a neutrino in a flavour state $\beta$ after propagating a distance $L$ and being produced as a flavour state $\alpha$ is given by:

$$
\begin{aligned}
P_{\alpha \rightarrow \beta}=\delta_{\alpha \beta} & -4 \sum_{j>i} \operatorname{Re}\left[U_{\alpha i}^{*} U_{\beta i} U_{\alpha j} U_{\beta j}^{*}\right] \sin ^{2}\left(\left[\frac{1.27 \mathrm{GeV}}{\mathrm{eV}^{2} \mathrm{~km}}\right] \frac{\Delta m_{j i}^{2} L}{E}\right) \\
& +2 \sum_{j>i} \operatorname{Im}\left[U_{\alpha i}^{*} U_{\beta i} U_{\alpha j} U_{\beta j}^{*}\right] \sin \left(\left[\frac{2.54 \mathrm{GeV}}{\mathrm{eV}^{2} \mathrm{~km}}\right] \frac{\Delta m_{j i}^{2} L}{E}\right),
\end{aligned}
$$

where $U$ is the neutrino mixing matrix, $\mathrm{E}$ is the neutrino energy and $\Delta m_{j i}^{2}=m_{j}^{2}-m_{i}^{2}$ [10]. The corresponding antineutrino oscillation probability can be obtained by replacing the unitary neutrino mixing matrix $U \rightarrow U^{+}$. A more rigorous treatment can be found in [11].

In the currently accepted neutrino model, there are three massive neutrinos leading to two distinct differences between the squared masses, $\Delta m_{21}^{2}$ and $\Delta m_{32}^{2}$. The $3 \times 3$ lepton mixing matrix, called the Pontecorvo-Maki-Nakagawa-Sakata (PNMS) matrix, connects the mass eigenstates to the weak interaction eigenstates:

$$
\left(\begin{array}{c}
v_{e} \\
v_{\mu} \\
v_{\tau}
\end{array}\right)=\left(\begin{array}{lll}
U_{e 1} & U_{e 2} & U_{e 3} \\
U_{\mu 1} & U_{\mu 2} & U_{\mu 3} \\
U_{\tau 1} & U_{\tau 3} & U_{\tau 3}
\end{array}\right)\left(\begin{array}{c}
v_{1} \\
v_{2} \\
v_{3}
\end{array}\right) .
$$

This mixing can be parametrised with three mixing angles and one CP violating phase $\delta_{C P}$. The best measured values of the parameters in the neutrino sector are obtained from global fits and found in the latest edition of the Review of Particle Physics [12] and listed in Table 2.1.

The neutrino mass hierarchy - the ordering between $m_{2}$ and $m_{3}$ - and $\delta_{C P}$ are currently still not well determined. One way to obtain sensitivity to both of these is through the Mikheyev-Smirnov-Wolfenstein effect. The presence of matter affects neutrino propagation. When a neutrino propagates through a dense medium, its energy-momentum relation is modified by coherent interactions with background particles. Because earthly matter does not contain muons and taus, the Charged Current (CC) interactions (see Section 2.5) 
Table 2.1: $3 v$ oscillation parameters obtained from [13]. All five parameters are separately given with $1 \sigma$ intervals for both the case normal ordering or inverse ordering is assumed.

\begin{tabular}{lcc}
\hline & Normal Ordering & Inverse Ordering \\
\hline $\sin ^{2} \theta_{12}$ & $0.320_{-0.016}^{+0.020}$ & $0.32_{-0.016}^{+0.020}$ \\
$\sin ^{2} \theta_{23}$ & $0.547_{-0.030}^{+0.020}$ & $0.551_{-0.030}^{+0.018}$ \\
$\sin ^{2} \theta_{13}$ & $\left(2.160_{-0.069}^{+0.083}\right) 10^{-2}$ & $\left(2.220_{-0.076}^{+0.074}\right) 10^{-2}$ \\
$\Delta m_{21}^{2}$ & $\left(7.55_{-0.16}^{+0.20}\right) 10^{-5} \mathrm{eV}^{2}$ & $\left(7.55_{-0.16}^{+0.20}\right) 10^{-5} \mathrm{eV}^{2}$ \\
$\Delta m_{32}^{2}$ & $\left(2.42_{-0.03}^{+0.03}\right) 10^{-3} \mathrm{eV}^{2}$ & $\left(-2.50_{-0.03}^{+0.04}\right) 10^{-3} \mathrm{eV}^{2}$ \\
\hline
\end{tabular}

with the medium only affect electron neutrino propagation. This flavour dependence leads to a measurable change in the ratio between neutrino interactions of the different flavours. The evident way to exploit the MSW effect are long-baseline neutrino experiments. Deep Underground Neutrino Experiment (DUNE) is an international experiment dedicated to the search for leptonic charge-parity symmetry violation and the neutrino mass hierarchy problem. To attain this goal, DUNE will look at a high intensity muon beam with a near detector at Fermilab and a far detector, the latter being positioned $1300 \mathrm{~km}$ from the beam origin. Before DUNE turns on, several other neutrino experiments such as $\mathrm{T} 2 \mathrm{~K}, \mathrm{NO} v \mathrm{~A}$, ICECUBE and JUNO will already constrain the available parameter space for $\delta_{C P}$ and the mass hierarchy [14]. 


\subsection{The sterile neutrino hypothesis}

Most of the oscillation results are well described by the three $v$ generations hypothesis. However, there are a few anomalous indications, the so-called short-baseline anomalies, that cannot be explained with this model. The anomalies could indicate the existence of at least one additional neutrino with a mass of around $1 \mathrm{eV}$, called a sterile neutrino [15]. Sterile, because several indications show that there are three light active neutrinos, where active refers to the particle taking part in weak interactions. One of these indications is the measurement of the invisible width of the $Z$ boson at LEP. The result, giving the number of neutrinos to be $2.92 \pm 0.05$, means that additional neutrinos must lack weak interactions [1]. They would be observable only when mixing with the familiar active neutrinos.

\subsubsection{The simplest $3+1$ extension}

A fourth - sterile - neutrino flavour, with a fourth corresponding mass eigenstate, can be added to the PNMS matrix:

$$
\left(\begin{array}{l}
v_{e} \\
v_{\mu} \\
v_{\tau} \\
v_{S}
\end{array}\right)=\left(\begin{array}{llll}
U_{e 1} & U_{e 2} & U_{e 3} & U_{e 4} \\
U_{\mu 1} & U_{\mu 2} & U_{\mu 3} & U_{\mu 4} \\
U_{\tau 1} & U_{\tau 2} & U_{\tau 3} & U_{\tau 4} \\
U_{S 1} & U_{S 2} & U_{S 3} & U_{S 4}
\end{array}\right)\left(\begin{array}{c}
v_{1} \\
v_{2} \\
v_{3} \\
v_{4}
\end{array}\right)
$$

Since this neutrino does not interact through the strong or electroweak interaction, there are only two methods to probe its existence. First, through gravitational interaction, this sterile neutrino could be a dark matter candidate. Nonetheless, the cosmological constraints on its abundance and mass are strongly model dependent [12]. Second, such a sterile neutrino would still affect the oscillation pattern of the three active flavours by introducing an additional mass splitting into the system. Experimental data that will be discussed in the next paragraphs indicate that the two known mass splittings are small compared to the third. Therefore, the $3+1$ sterile neutrino extension is hierarchical, consisting of three active states that are well separated from an additional sterile state. Note that this $3+1$ extension is only 
the most simple form and that the existence of additional sterile neutrinos is not ruled out, as long as their mass spitting is large compared to the active states.

The anomalies described in this section hint towards additional oscillations corresponding with a squared mass splitting of the order of $1 \mathrm{eV}^{2}$. The two splittings associated with the three neutrino sector are on the order of $10^{-5} \mathrm{eV}^{2}$ and $10^{-3} \mathrm{eV}^{2}$ [13]. The effect of the two small splittings on an experiment designed to look for $\mathcal{O}\left(1 \mathrm{eV}^{2}\right)$ scale oscillations will be negligible. Therefore, we use the short-baseline approximation, where we assume that the mass eigenstates that participate in the standard oscillations are degenerate (i.e. $\Delta m_{21}^{2}=0$ and $\Delta m_{32}^{2}=0$ ). The heavier sterile neutrino would be responsible for additional oscillations at a value of $L / E$ much lower than the atmospheric and solar oscillations. The oscillation probability for $v_{\alpha} \rightarrow v_{\beta}$, Equation (2.11), in the $3+1$ model reduces to [16]:

$$
P_{\alpha \rightarrow \beta}=\delta_{\alpha \beta}-4\left(\delta_{\alpha \beta}-U_{\alpha 4} U_{\beta 4}^{*}\right) U_{\alpha 4}^{*} U_{\beta 4} \sin ^{2}\left(\left[\frac{1.27 \mathrm{GeV}}{\mathrm{eV}^{2} \mathrm{~km}}\right] \frac{\Delta m_{41}^{2} L}{E}\right) .
$$

This is valid if $E / L \gtrsim \Delta m_{41}^{2}$ and $\Delta m_{41}^{2}>>\Delta m_{21}^{2}, \Delta m_{32}^{2}$. For longer baselines, or lower energies, this approximation does not hold as the well known solar driven oscillation and atmospheric driven oscillation come into play. The latter two are characterised by the two mass splittings listed in Table 2.1. With any particular selection of $\alpha$ and $\beta$ this can be seen to be equivalent to a two neutrino model with a mixing amplitude of

$$
\sin ^{2} 2 \theta_{\alpha \beta}=\left|4\left(\delta_{\alpha \beta}-U_{\alpha 4} U_{\beta 4}^{*}\right) U_{\alpha 4}^{*} U_{\beta 4}\right|
$$

Furthermore, due to the high rest mass of the $\tau$-lepton $(1.8 \mathrm{GeV})$, it cannot be directly produced in reactor or accelerator neutrino sources. For electron and muon neutrino sources, the sterile oscillation can be parametrised by $U_{e 4}, U_{\mu 4}$ and $\Delta m_{41}^{2}$. Therefore, the three channels that are experimentally accessible are: 


$$
\begin{array}{crrr}
P\left(v_{e} \rightarrow v_{e}\right) & =1-4\left(1-\left|U_{e 4}\right|^{2}\right)\left|U_{e 4}\right|^{2} \sin ^{2}\left(1.27 \Delta m_{41}^{2} \frac{L}{E}\right) & \left(v_{e} \text { disappearance }\right) \\
P\left(v_{\mu} \rightarrow v_{\mu}\right)= & 1-4\left(1-\left|U_{\mu 4}\right|^{2}\right)\left|U_{\mu 4}\right|^{2} \sin ^{2}\left(1.27 \Delta m_{41}^{2} \frac{L}{E}\right) & \left(v_{\mu} \text { disappearance }\right) \\
P\left(v_{\mu} \rightarrow v_{e}\right)= & 4\left|U_{e 4}\right|^{2}\left|U_{\mu 4}\right|^{2} \sin ^{2}\left(1.27 \Delta m_{41}^{2} \frac{L}{E}\right) & \left(v_{e} \text { appearance }\right)
\end{array}
$$

Each of these channels has a corresponding antineutrino version by the substitution $v_{\alpha} \leftrightarrow$ $\bar{v}_{\alpha}$. This demonstrates that there are two experimental methods to search for neutrino oscillations: the "disappearance" method and the "appearance" method. In the disappearance channels, one looks at the change in event rate of the produced neutrino flavour over a certain baseline at a specific energy. The ability to perform such a measurement is limited by the knowledge of the neutrino flux of the source and the interaction cross section. Appearance searches look for $v_{\beta}$ events in a source dominated by $v_{\alpha}$. This method depends on knowledge of the intrinsic contamination of $v_{\beta}$ in the source and the ability to reject background events originating from $v_{\alpha}$ interactions. 


\subsection{Short-baseline anomalies}

A series of short-baseline neutrino oscillation experiments provided unexpected results. The results they obtained are inconsistent or in strong tension with the predictions obtained from the three neutrino oscillation paradigm. In this section we divide them into three categories:

- Accelerator neutrino experiments

- Radio-chemical experiments

- Nuclear reactor experiments.

\subsubsection{Accelerator neutrino experiments}

The Liquid Scintillator Neutrino Detector (LSND) observed an excess of $\bar{v}_{e}$ events from a well understood $\bar{v}_{\mu}$ source in 2001 [17]. At the same period, the KARMEN experiment studied neutrinos supplied via the decay of pions produced when a proton beam strikes a target. It operated from 1990 until March 2001, searching for the appearance and disappearance of electron neutrinos. KARMEN did not observe any oscillation signal. Limits were set on neutrino oscillation parameters [18], which are in tension with the LSND experiment and were followed up by MiniBooNE.

\section{Liquid Scintillator Neutrino Detector}

The first tension with the three-neutrino framework arose in the 1990s by the LSND experiment at Los Alamos [19]. The neutrino beam was produced by firing a $0.8 \mathrm{GeV}$ proton beam into a target. The charged pions produced are stopped in the beam dump where they decay at rest. As illustrated in Figure 2.4, this leads to a well understood source of muon antineutrinos of $0 \mathrm{MeV}$ to $53 \mathrm{MeV}$.

A liquid scintillator detector was positioned $31 \mathrm{~m}$ away from the target. The process searched for was inverse beta decay:

$$
\bar{v}_{\mu} \stackrel{\text { oscillation }}{\longrightarrow} \bar{v}_{e}+p \rightarrow e^{+}+n
$$




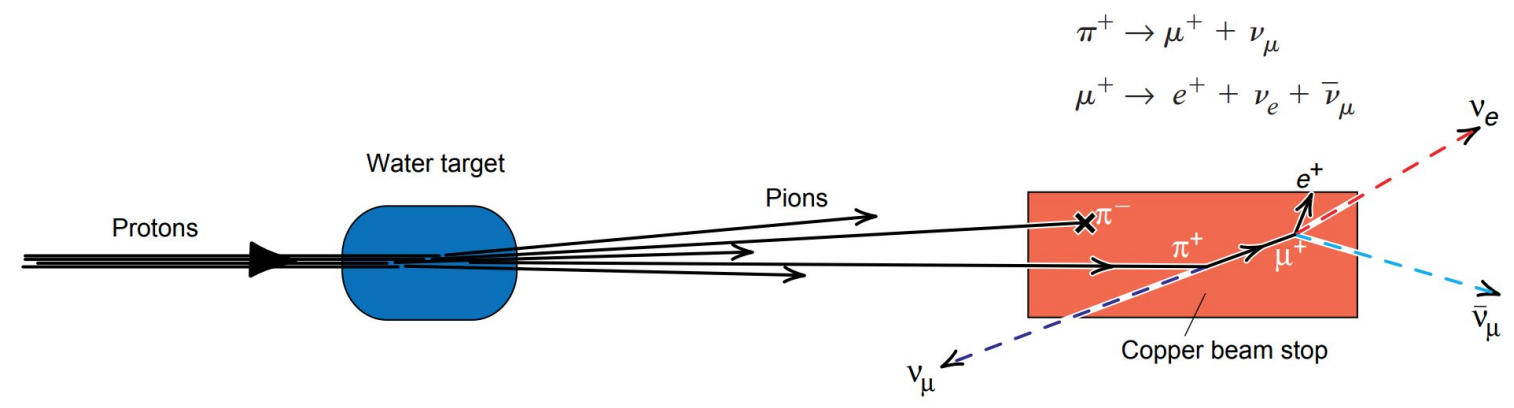

Figure 2.4: Neutrino beam used by the LSND experiment. A proton beam is fired at a water target, where they produce pions. Most of the pions travel half a meter in the air before striking a copper beam stop and coming to rest. Nearly all of the negative pions are absorbed by copper nuclei before they decay, but each positive pion decays at rest to a muon and a muon neutrino. The muons also come to rest in the beam stop and decay to a muon antineutrino, a positron, and an electron antineutrino. The neutrinos fly off in all directions isotropically. Illustration from [20].

The signature of such an event is illustrated on the left panel of Figure 2.5.

The LSND experiment reported an anomalous event excess in the $\bar{v}_{\mu} \rightarrow \bar{v}_{e}$ appearance channel, the result is shown on the right panel of Figure 2.5 [17]. This could be interpreted as an oscillation with $\Delta m^{2} \sim 1 \mathrm{eV}$ (see Equation (2.18)). Such a scale is clearly incompatible with $\Delta m_{21}^{2}$ and $\Delta m_{32}^{2}$ (see Table 2.1) and could be explained with the presence of sterile neutrinos $\left(\bar{v}_{\mu} \rightarrow v_{\text {sterile }} \rightarrow \bar{v}_{e}\right)$. At the same time, the results of the KARMEN and Bugey experiments excluded parts of the allowed low $\Delta m^{2}$ region $[18,21]$. Within the constraints of the former, the LSND signal is best fitted through an additional sterile neutrino oscillation with a mass splitting of the order of $1 \mathrm{eV}$ [15].

\section{MiniBooNE}

The MiniBooNE experiment, located at Fermilab, was designed with the goal to validate the LSND measurement [22]. The neutrino beam used will be the topic of Section 3.1.1. For current purposes, it is important to note that the Booster Neutrino Beam is able to run in both neutrino and antineutrino modes, and that the muon (anti)neutrino energy is peaked around $800 \mathrm{MeV}$. To be sensitive to the signal seen by LSND, the baseline was taken to be $541 \mathrm{~m}$, leading to a very similar $L / E$ value. 

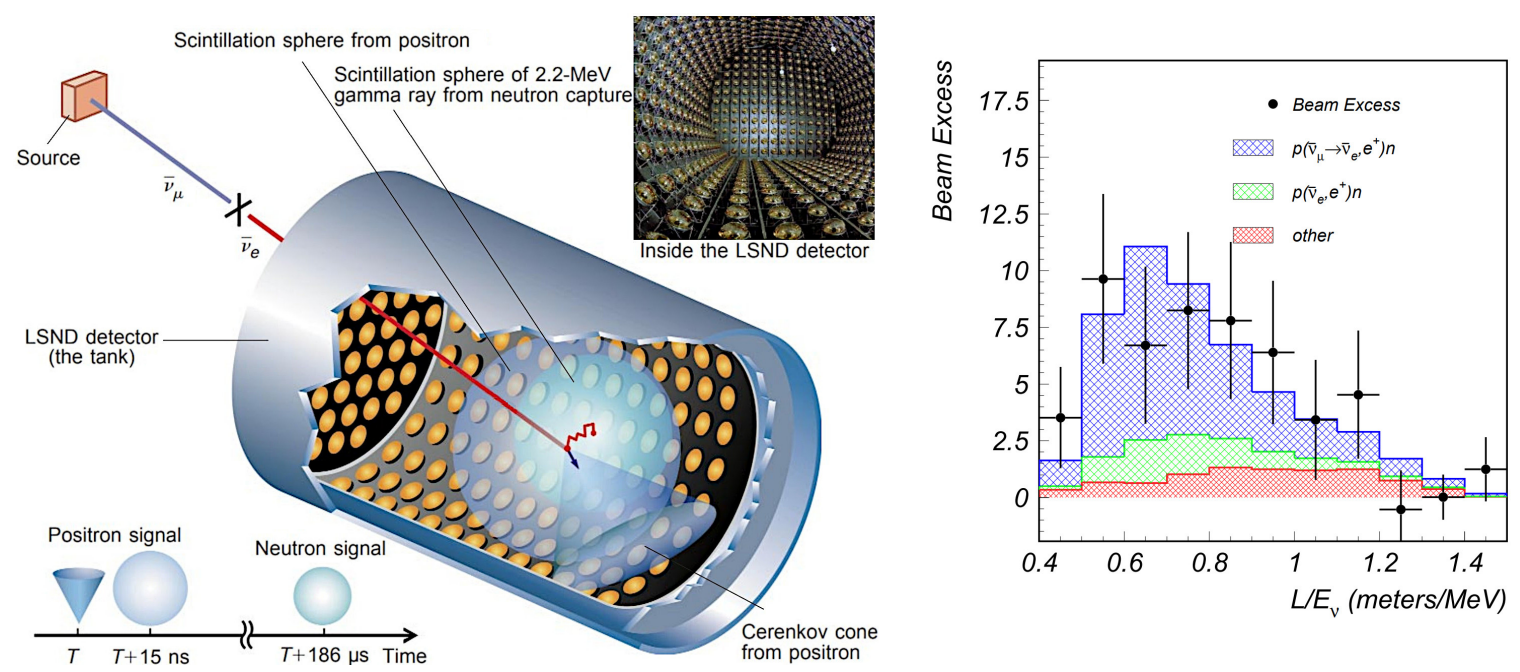

Figure 2.5: (Left) Signature of a $\bar{v}_{e}$ event in LSND. A muon antineutrino produced at the source oscillates en-route to the detector and appears as an electron antineutrino. The neutrino scatters of a free proton in the oil, creating a positron and a neutron. The positron travels faster than the speed of light in the oil and so produces a Cherenkov cone. As it loses energy through collisions with atoms in the oil, the positron also produces a sphere of scintillation light. The neutron survives about 186 microseconds and wanders $\mathcal{O}(1 \mathrm{~m})$ before it is absorbed by a nucleus, emitting a $2.2 \mathrm{MeV}$ gamma ray that also produces a sphere of scintillation light. This succession of events is the signature of an electron antineutrino. (Right) The observed excess of candidate $\bar{v}_{e}$ events. The blue part of the histogram is the contribution due to a potential additional oscillation. Illustration adapted from [20,17]

The detector consist of a $12 \mathrm{~m}$ diameter sphere filled with 10 million litres of mineral oil. The active volume is surrounded by a sphere, instrumented with 12808 inch PMTs. An additional 240 PMTs are located in the outer veto region and used for cosmic activity rejection. Particles travelling through the mineral oil at a speed exceeding the speed of light in the medium will emit Cherenkov light. The kinetic energy threshold for Cherenkov production depends on the mass of the particle, making MiniBooNE fairly insensitive to protons and neutrons compared to light particles such as electrons and muons. Between the latter two, particle identification is made based on the shape of the Cherenkov cone captured by the PMT system, as illustrated in Figure 2.6.

Apart of particle observance through the direct emission of Cherenkov light, detection can happen through secondary processes. Due to the nature of electromagnetic shower formation - fully introduced in Section 8.3.1 - high energetic photons will undergo scattering 


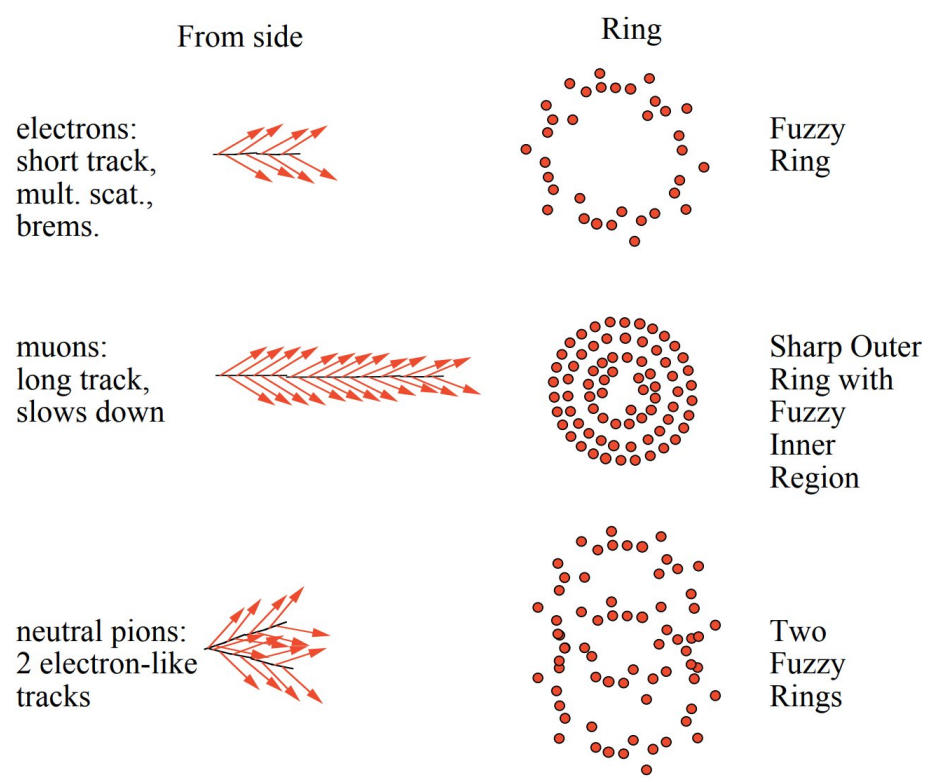

Figure 2.6: Particle Identification in MiniBooNE. Schematic showing the different signatures of event topologies as seen by the PMT system of MiniBooNE. Electrons scatter, creating a fuzzy ring. Muons follow a more straight trajectory, leading to a sharper ring structure. The photons from $\pi^{0} \rightarrow \gamma \gamma$ decays produce electrons through scattering an therefore creating similar signatures. Figure from [23].

and pair-production. The produced electrons - and positrons - in these processes emit Cherenkov light if their energy is above threshold. Therefore, the signature of photons and electrons is indistinguishable in MiniBooNE. Neutrons could, in principle, be detected by 2.2 MeV $\gamma^{\prime}$ s that result from delayed (lifetime of $186 \mu \mathrm{s}$ ) neutron capture on protons within the mineral oil. In practice, this is below the detection threshold arising from radioactivity, PMT noise etc.

For energy calibration, MiniBooNE relied on the typical Michel electron spectrum peaked at $m_{\mu} / 2=52.8 \mathrm{MeV}$. Furthermore, the invariant mass from the $\pi^{0} \rightarrow \gamma \gamma$ was also reconstructed to validate the energy response around $135 \mathrm{MeV}$.

The interaction looked for by the MiniBooNE sterile neutrino search was:

$$
\begin{array}{lr}
v_{\mu} \stackrel{\text { oscillation }}{\longrightarrow} v_{e}+n \rightarrow e^{-}+p & \text { (neutrino mode) } \\
\bar{v}_{\mu} \stackrel{\text { oscillation }}{\longrightarrow} \bar{v}_{e}+p \rightarrow e^{+}+n & \text { (antineutrino mode) }
\end{array}
$$


Here, the final state electron from the Charged Current (CC) neutrino scattering is observed through a fuzzy Cherenkov ring signature. Being a different detector technology, the systematic uncertainties of MiniBooNE are completely different from those of the LSND experiment. Nonetheless, having a similar $L / E$ value and sensitivity to the same neutrino interaction channel, MiniBooNE can test $\mathcal{O}(1 \mathrm{eV})$ sterile neutrino hypothesis.

After 15 years of data-taking, the MiniBooNE experiment reported results from an analysis of $v_{e}$ appearance data from $12.84 \times 10^{20} \mathrm{POT}$ in neutrino mode. If interpreted as an excess of $v_{e}$ charged-current quasielastic events, the size of the excess is $381.2 \pm 85.2$, corresponding to $4.5 \sigma$ in the energy range $200 \mathrm{MeV}$ to $1250 \mathrm{MeV}$. Combining these data with the $\bar{v}_{e}$ appearance data from $11.27 \times 10^{20}$ POT in antineutrino mode, a total of $\left(v_{e}+\bar{v}_{e}\right)$ charged-current quasielastic event excess of $460.5 \pm 99.0$ events $(4.7 \sigma)$ is observed. The MiniBooNE data are consistent in energy and magnitude with the excess of events reported by LSND, and the significance of the excesses of the two experiments combined is $6.0 \sigma$ [23]. The final result in neutrino mode is shown in the left panel of Figure 2.7. The right panel investigates the compatibility of the LSND and MiniBooNE in the light of the sterile neutrino hypothesis.
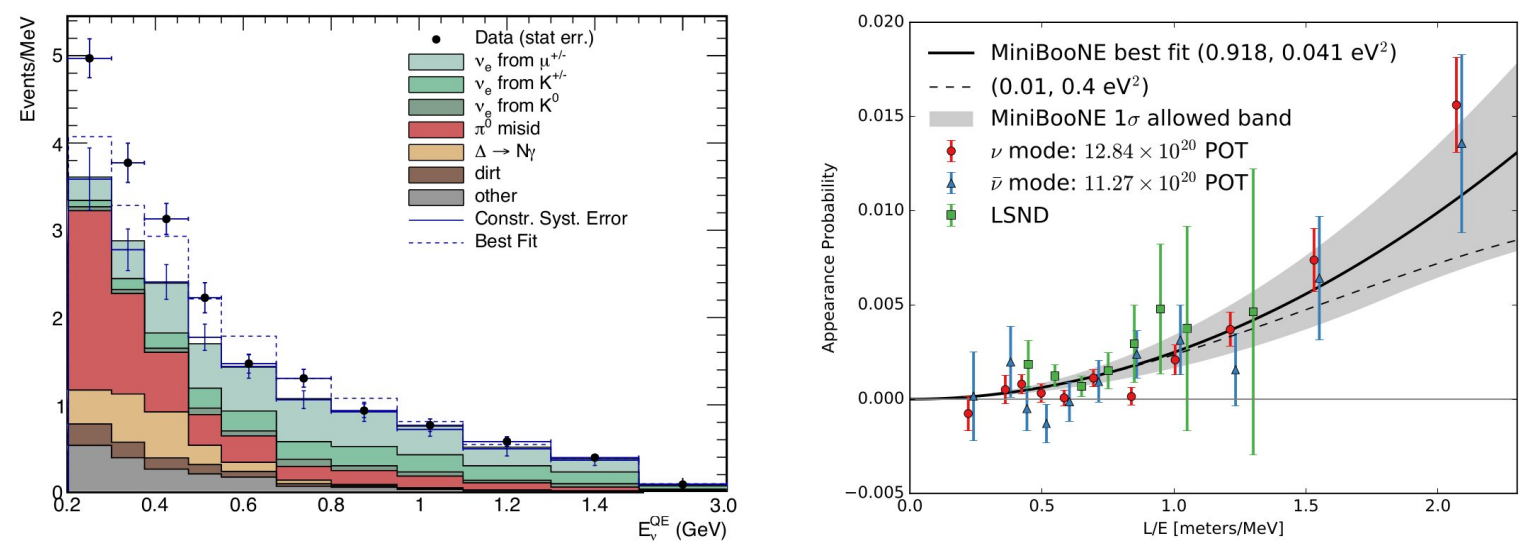

Figure 2.7: (Left) The low-energy excess as observed by MiniBooNE in neutrino mode. The reconstructed neutrino energy under the hypothesis that the charged-current interaction is quasielastic. The excess in data is clearly visible at low energies. The dotted line corresponds to the prediction when a sterile neutrino is included in the model. (Right) The allowed parameter space by the LSND and MiniBooNE (in neutrino and antineutrino mode) for a sterile neutrino. Figure from [23].

It is important to stress the quasi-elastic hypothesis in the MiniBooNE result. The selected 
events are interpreted under the assumption of scattering with a single free nucleon. In the case of an electron neutrino interaction, the energy can be reconstructed using the final state lepton:

$$
E_{v}^{Q E}=\frac{2 m_{n} E_{e}+m_{p}^{2}-m_{n}^{2}-m_{e}^{2}}{2\left(m_{n}-E_{e}+\cos \theta \sqrt{E_{e}^{2}-m_{e}^{2}}\right)},
$$

where $m_{n}, m_{p}$ and $m_{e}$ are the mass of the neutron, proton and the electron. $\theta$ is the angle of the outgoing lepton with respect to the incoming neutrino direction. In reality, intranuclear processes such as pion absorption complicate the energy reconstruction, as will be discussed in Section 2.5. It is therefore possible that the energy reconstruction of the observed excess events is underestimated and corresponds to higher true neutrino energies.

MiniBooNE Backgrounds In the low-energy region where the excess manifests itself, three background categories can mimic the Low Energy Excess (LEE) as observed by MiniBooNE

- Intrinsic $v_{e}$ :

The $v_{e}$ component of the beam, coming from $\mu^{ \pm}, K^{ \pm}$, and $K^{0}$, is the irreducible background of the experiment, since it can't be distinguished from $v_{\mu}$ oscillating into $v_{e}$. This component of the flux is partially constrained by measuring the $v_{\mu}$ interactions.

- Misidentified $\pi^{0}$ :

The background from misidentified $\pi^{0}$ events represents the largest component. These events are particularly challenging to reconstruct since very forward-boosted photons will appear in the detector as a single fuzzy ring. The MiniBooNE collaboration has constrained this contribution by reconstructing the invariant $\pi^{0}$ mass of the event and obtaining a sample with a purity $>90 \%$ of NC $\pi^{0}$ events. The total uncertainty on the NC background is 7\% [24].

- Misidentified $\Delta \rightarrow N \gamma$

A neutral current resonant interaction can produce a $\Delta$ resonance, which has a rare electromagnetic decay channel $\Delta \rightarrow N \gamma$, where $N=n, p$. This channel is also constrained by the NC $\pi^{0}$ in-situ measurement. The uncertainty on this component is $12 \%$ [24]. 
Both the $\pi^{0}$ and the $\Delta \rightarrow N \gamma$ backgrounds arise from the inability of a Cherenkov detector to distinguish between photons and electrons, which is one of the most powerful capabilities of a LArTPC, as will be discussed in the next chapter.

\subsubsection{Radio-chemical neutrino detection experiments}

Two solar neutrino experiments, GALLEX [25] and SAGE [26], used intense neutrino sources $\left({ }^{51} \mathrm{Cr},{ }^{37} \mathrm{Ar}\right)$ during the detector calibration. These sources decay through electron capture, producing an electron neutrino and emitting $\gamma$-radiation. The sources were shielded to stop the gamma-radiation and act as a clean neutrino source. Both GALLEX and SAGE observed an $\approx 24 \%$ event deficit in the $v_{e}$ disappearance channel. This deficit is often referred to as the Gallium anomaly and can also be understood by neutrino oscillations with $1 \mathrm{eV}$ sterile neutrinos. A fit of the data in terms of neutrino oscillations favours at about $2.3 \sigma$ short-baseline electron neutrino disappearance with respect to the null hypothesis of no oscillations [27, 28].

\subsubsection{Reactor Antineutrino Anomaly (RAA)}

In 2011 Mueller et al. [29] as well as Huber [30] published new nuclear reactor antineutrino reference spectra computed from electron spectrum measurements but with revised conversion techniques. Both found a 3\% increase in the reactor flux compared to the previous predictions, which had been applied as standard for more than 20 years. A reanalysis of the measured versus the predicted neutrino flux was performed using the antineutrino flux measurements at $100 \mathrm{~m}$ baseline and less. Together with an updated neutrino interaction cross section, the computation resulted in an observed-to-predicted ratio of $0.935 \pm 0.024$ [31], which is the so-called Reactor Antineutrino Anomaly (RAA) [32].

Figure 2.8 displays the data of several reactor experiments together with two hypothetical fits, one in the case of three neutrinos, the other includes one sterile neutrino. Clear preference is given to the $3+1$ neutrino hypothesis.

The differences found in the short-baseline reactor experiments could indicate unaccounted 


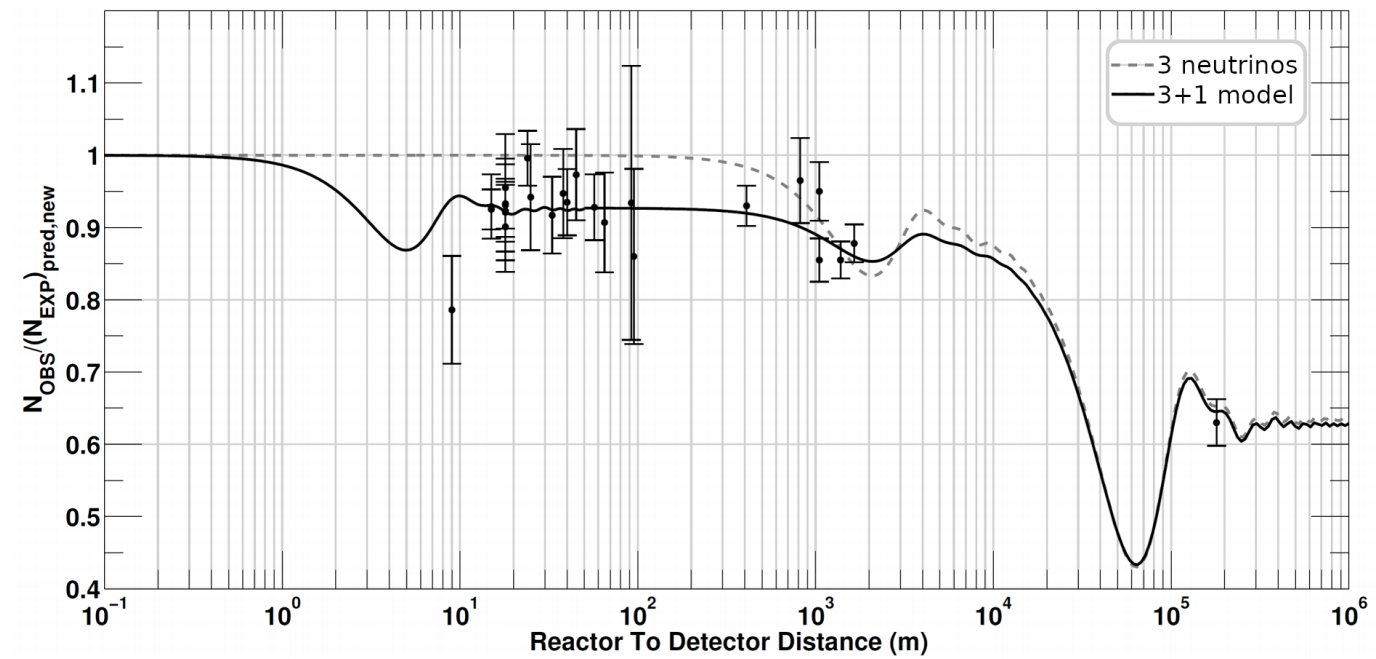

Figure 2.8: Illustration of the short-baseline reactor antineutrino anomaly. The graph shows the ratio of the observed to the expected rate, from the recent reactor neutrino flux calculations without neutrino oscillations, for all reactor neutrino experiments at various baselines. The dashed line corresponds to the classic 3 neutrino oscillation scenario, while the solid line corresponds to the $3+1$ model ( 3 active neutrinos +1 sterile neutrino) with $\Delta m^{2} \approx 1 \mathrm{eV}$. The bump around $3 \mathrm{~km}$ is due to the well-measured atmospheric neutrino oscillation, the one around $100 \mathrm{~km}$ is the solar neutrino oscillation. Figure from [33].

physics in the propagation and detection of neutrinos, such as sterile neutrinos. On the contrary the differences could also be introduced by errors on the predicted reactor spectra. Upcoming very short-baseline experiments will test if the discovered overall deficit in antineutrino rate is linked to neutrino flavour oscillations into a light sterile state. Even without new physics, data from different reactor types will bring valuable insights into the nature of the reactor shape distortion, bypassing the use of summation spectra and, accordingly, their large uncertainties. Among these undertakings worldwide, SoLiz [34], a very short-baseline reactor experiment at the BR2 research reactor in Belgium, will test whether the sterile neutrino hypothesis is realistic or not by searching for a distance dependent deficit.

\subsubsection{Current status \& global fits}

Combined with cosmological evidence for an additional relativistic degree of freedom before recombination $[35,36,37]$, the gallium, LSND/MiniBooNE neutrino disappearance 
anomalies and the Reactor Antineutrino Anomaly provide substantial arguments for sterile neutrinos. An overview of the significance of these anomalies is given in Table 2.2. Even though hints for a sterile neutrino are observed in several channels by a large set of experiments employing widely varying detector technologies, the picture is far from clear. The lack of observation of a sterile neutrino oscillation in the muon disappearance channels creates tension between different data sets.

Table 2.2: Significance of experimental anomalies from neutrino experiments. LSND and MiniBooNE are accelerator-based short-baseline experiments. GALLEX/SAGE are radioactive source experiments. The others are reactor experiments and represent the Reactor Antineutrino Anomaly (RAA). A detailed overview of the anomalies and their significance is given in [38].

\begin{tabular}{lll}
\hline Experiment & Channel & Significance \\
\hline LSND & $v_{\mu} \rightarrow v_{e}$ & $3.8 \sigma$ \\
MiniBooNE & $v_{\mu} \rightarrow v_{e}$ & $4.5 \sigma$ \\
MiniBooNE & $\bar{v}_{\mu} \rightarrow \bar{v}_{e}$ & $2.8 \sigma$ \\
GALLEX/SAGE & $v_{e}$ disappearance & $2.3 \sigma$ \\
Reactors & $\bar{v}_{e}$ disappearance & $2.7 \sigma$ \\
\hline
\end{tabular}

Global fits have found a large improvement in the $\Delta \chi^{2}=\chi_{n u l l}^{2}-\chi_{3+1}^{2}$, indicating the data favours a correction that behaves like an additional oscillation [39]. An internal inconsistency arises when the goodness of fit is evaluated on the appearance and disappearance data-sets separately. There are several possible explanations for the tension:

1. There are no sterile neutrinos. In this case, the separate data-sets must suffer from biases and unevaluated systematic uncertainties. Those biases, over different channels and detection technologies accidentally match the effect produced by a sterile neutrino.

2. There is one light sterile neutrino as explained in the simplest $3+1$ extension. In this scenario, some data-sets suffer from unidentified experimental effects. In the case of MiniBooNE, for example, the result is systematic limited, increasing the likeliness of unaccounted uncertainties in the result. Furthermore, it is worth noting that null results providing limits, such as the disappearance searches, have received less scrutiny. 
3. There is additional new physics in the neutrino sector. More complicated models such as a decaying sterile neutrino or a $3+\mathrm{N}$ model introduce additional degrees of freedom and are able to relieve tension in the data.

From a theoretical point of view, it is worth noting that, light sterile neutrinos do not show up in a natural way in most beyond the Standard Model theories. The popular type-I seesaw model, for example, which provides an explanation of the small neutrino couplings, predicts only heavy sterile neutrinos $\left(m>10^{10} \mathrm{GeV}\right)$. If light sterile neutrinos indeed exist, they would suggest new frontiers in both experimental and theoretical physics. 


\subsection{Neutrino interactions}

This section introduces the complexities of neutrino-nucleus scattering with the focus on argon, the atom of interest for the MicroBooNE experiment. The interaction is approximated as a two-step process. In the first step, the neutrino interacts with one or two bound nucleons in the nucleus. The nucleons are approximated to be quasi-free and the final state interactions can be treated independently as a second step. We will first summarise these nuclear effects and then describe four neutrino interaction modes of neutrino scattering with bound nucleons in the nucleus. All the described characteristics and processes have a direct impact on our ability to reconstruct the energy of the incoming neutrino using the properties of the final state particles.

\subsubsection{Nuclear effects}

Neutrinos interact with nucleons that are aggregated in nuclei and therefore nuclear effects alter the interactions and the products beyond the nucleon-neutrino expectation. In general these can be split up into two categories:

- Initial state effects originating from the nucleon-nucleon interactions in the atom.

- Final state effects originating from the passage of the neutrino interaction products through the nuclear matter to exit the nucleus.

The technology employed for the MicroBooNE experiment requires noble gasses with complex nuclei such as argon, consisting of 18 protons and 22 neutrons. When dealing with complex nuclei, nuclear effects cannot be neglected and have an important impact on the cross-section modelling and energy reconstruction. At the time of writing, the understanding of neutrino-argon scattering is poorly understood. Therefore, it is one of the major physics goals of the MicroBooNE experiment to measure and study cross-sections. This knowledge will be crucial for future experiments using argon as target medium.

Fermi motion The nucleons inside the nucleus are not at rest. The momentum distribution of the nucleons varies depending on the approximation used and the magnitude of the ef- 
fect is typically of the order of $0.2 \mathrm{GeV}$. In MicroBooNE, different models are compared,such as the Bodek-Ritchie Fermi Gas, the Local Fermi Gas and the Effective Spectral Function approximation [40]. This initial nucleon momentum shifts the centre of mass frame in which the $v$-nucleon scattering takes place, effectively smearing the neutrino energy reconstruction.

Final State Interactions (FSI) affect the hadrons produced in the first step of the neutrinonucleon scattering and happen as the pions and protons re-scatter before exiting the nucleus. Figure 2.9 sketches different FSI processes.

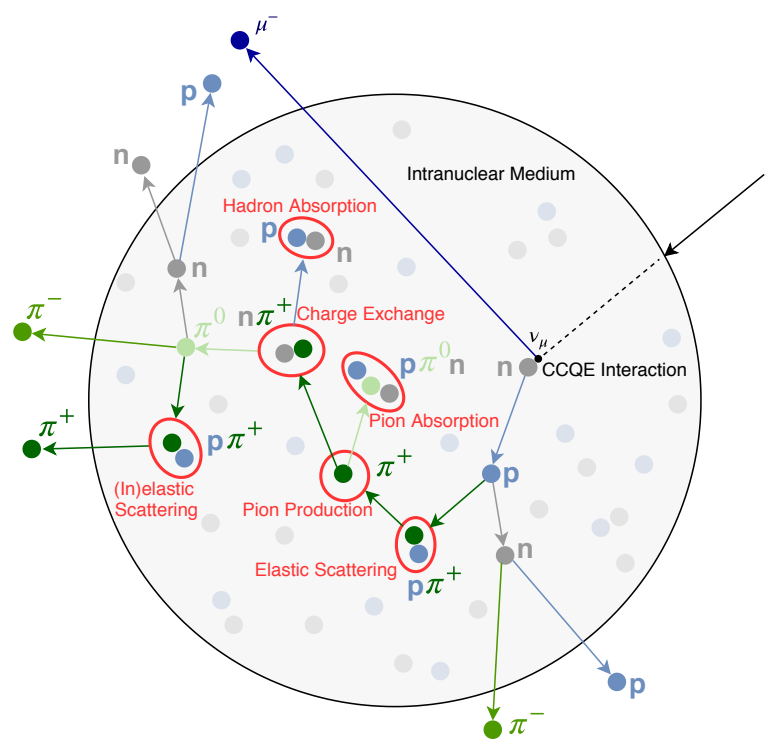

Figure 2.9: Example of a muon neutrino charged current interaction on an argon nucleus. After the neutrino scatters of a quasi free nucleon (a neutron in this example), the muon leaves the nucleus. The produced proton - or more complex hadronic system - still needs to exit the nucleus. While traversing the dense nuclear matter, they are subject to Final State Interactions (FSI) before appearing in the detector. Examples of these are hadron absorption, charge exchange, pion production/absorption, elastic scattering and inelastic scattering. Figure from [41]

As a result of all these processes, the products of neutrino interactions can include a variety of final states with corresponding emission of nucleons and produced outgoing particles such as pions, kaons and other mesons. Also the outgoing lepton is impacted by the complexity of the positively charged target nucleus. Positively charged leptons undergo an 
additional acceleration, while negatively charged leptons experience a deceleration.

\subsubsection{Neutrino interaction modes}

This section will introduce four types of neutrino scattering processes that play a role in the MicroBooNE experiment. Emphasis will be put on Charged Current (CC) interactions where a charged $W$ boson is the mediator for the weak interaction, as seen in the Feynman diagrams in Figures 2.10 to 2.13. CC interactions are characterised by an outgoing lepton in the final state. Analogue Neutral Current (NC) processes take place and can be obtained by replacing the charged mediator by the neutral Z boson. In Neutral Current processes, there will be an undetectable outgoing neutrino instead of the outgoing charged lepton.

\section{Quasi-Elastic scattering (QE)}

At neutrino energies below $\approx 1.5 \mathrm{GeV}, \mathrm{QE}$ interactions are the dominant type. Here, neutrinos scatter off an entire nucleon, liberating the nucleon from the target - or multiple nucleons through final state interactions. The CC QE process is:

$$
v_{l}+n \rightarrow l^{-}+p, \quad \bar{v}_{l}+p \rightarrow l^{+}+n
$$

for a neutrino flavour $l$. This mode is illustrated in Figure 2.10.

\section{Meson Exchange Current (MEC)}

A specific set of intra-nuclear forces can be modelled by virtual meson exchange, usually between two nucleons, although bound states of more than two nucleons are possible. The so-called 2 particle- 2 hole ( $2 \mathrm{p}-2 \mathrm{~h})$ effect is important for neutrino scattering in the $\mathcal{O}(1 \mathrm{GeV})$ region where MicroBooNE is active. The $2 \mathrm{p}-2 \mathrm{~h}$ effect is dominated by the Meson Exchange Current (MEC), and is accompanied by a 2-nucleon emission from the primary vertex, instead of a single nucleon emission from the charged-current quasi-elastic interaction (see Figure 2.11). 

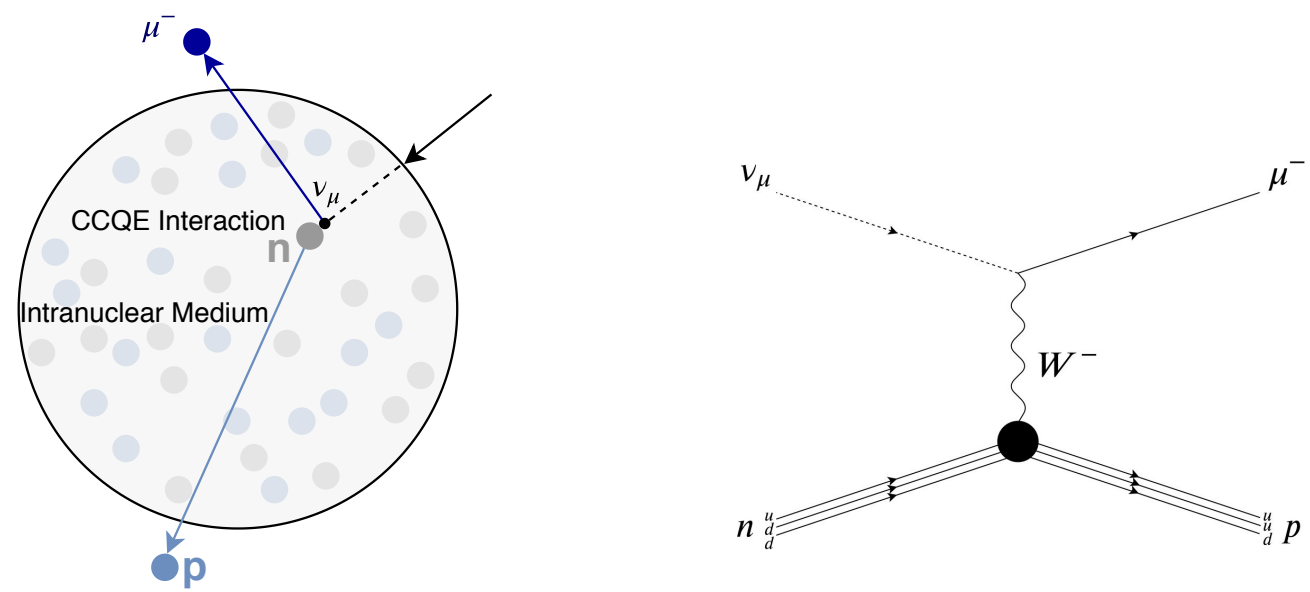

Figure 2.10: (Left) CC QE scattering on argon, a muon neutrino scatters of a neutron, the outgoing proton exits the nucleus. FSI might lead to additional particles exciting the nucleus. (Right) The corresponding Feynman diagram of the process on the single nucleon. Figure from [41]

\section{Resonant production (RES)}

If the neutrino-nucleus centre of mass energy exceeds the mass of the delta baryon $(\Delta)$ in Neutral Current events, and the sum of the delta baryon and the outgoing lepton mass in Charged Current events, resonant production can take place. This process kicks in around $\approx 0.5 \mathrm{GeV}$ and is a major contribution to the total cross section from $1 \mathrm{GeV}$ to $4 \mathrm{GeV}$. During resonance production, the neutrino brings the struck nucleon into an excited state, a baryon resonance. The baryon resonance quickly decays, often producing a nucleon and a single pion in the final state, shown in the left panel of Figure 2.12. Other higher multiplicity modes are possible, leading to additional pions. At the low energies encountered throughout this work, the dominant baryon resonance is the $\Delta(1232 \pm 2) \mathrm{MeV}$, which consists of any combination of three up/down quarks and therefore can have any unit charge between -1 and +2 . For charged current neutrino scattering, the final state is more likely to contain a neutral or positively charged pion due to the presence of the final state negative lepton. For charged current antineutrino scattering, the same argument is valid and it is more likely to have a negatively charged pion than a positively charged pion. 

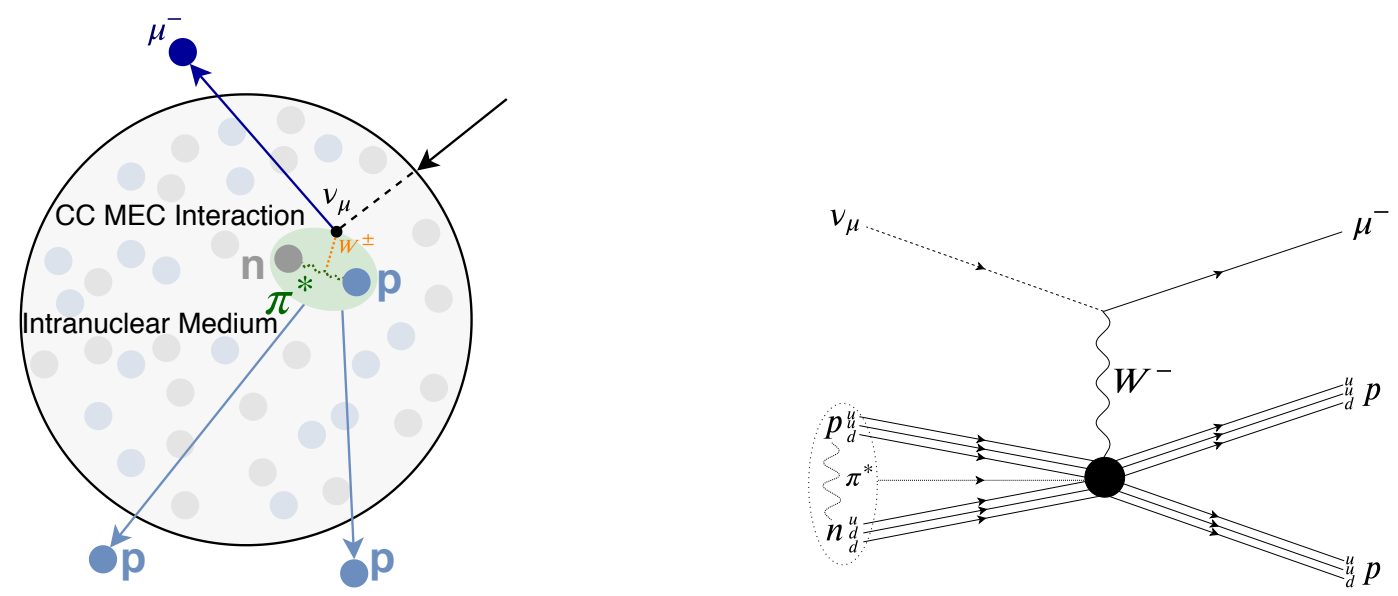

Figure 2.11: (Left) CC MEC scattering on argon, a muon neutrino scatters of a bounded two-nucleon system, the outgoing nucleons exit the nucleus. FSI might lead to additional particles exciting the nucleus. (Right) The corresponding Feynman diagram of the process. As an example, one proton and one neutron are bound through the exchange of a virtual charged pion. Figure from [41]

\section{Deep Inelastic Scattering (DIS)}

Deep Inelastic Scattering is the umbrella term for high-energy neutrino interactions - dominant above $\approx 4 \mathrm{GeV}$ - where the neutrino scatters of a quark instead the whole nucleus. This breaks apart the nucleon, producing a jet of hadrons. In MicroBooNE due to the lower neutrino beam energy, these events are fairly rare. The outgoing particles will heavily depend on final state interactions inside the nucleus.

\subsubsection{Neutrino interaction modelling in MicroBooNE}

In MicroBooNE, the default neutrino event generator is GENIE v3 [42]. GENIE combines a set of ab-initio calculations, phenomenological models and ad hoc additions to obtain a prediction of neutrino interactions on liquid argon in the $\mathcal{O}(1 \mathrm{GeV})$ region. The specific configuration employed in this thesis is the most current one as developed by the collaboration and is well-tested to reproduce results obtained by MiniBooNE, T2K, Minerva and $\mathrm{NO} v \mathrm{~A}$. The systematic uncertainties related to the modelling of the neutrino cross sections will be further discussed in Section 9.1.2. 

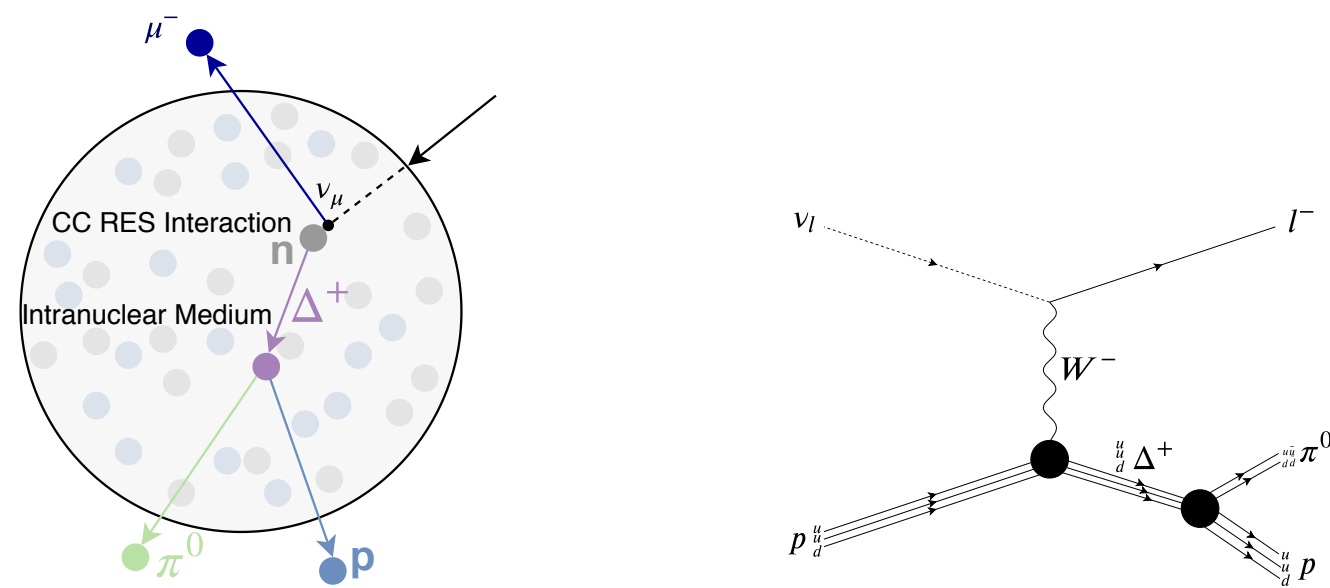

Figure 2.12: (Left) CC RES scattering on argon, a muon neutrino scatters of a single nucleon, producing an excited state, in this example a $\Delta^{+}$decaying to a $\pi^{0}$ and a proton. FSI might lead to additional particles exciting the nucleus. (Right) The corresponding Feynman diagram of the process. Note that this is just one example and that resonant production is possible with all neutrino flavours, neutral and charged current scattering, different baryonic excitations and a variety of decay channels. Figure from [41]
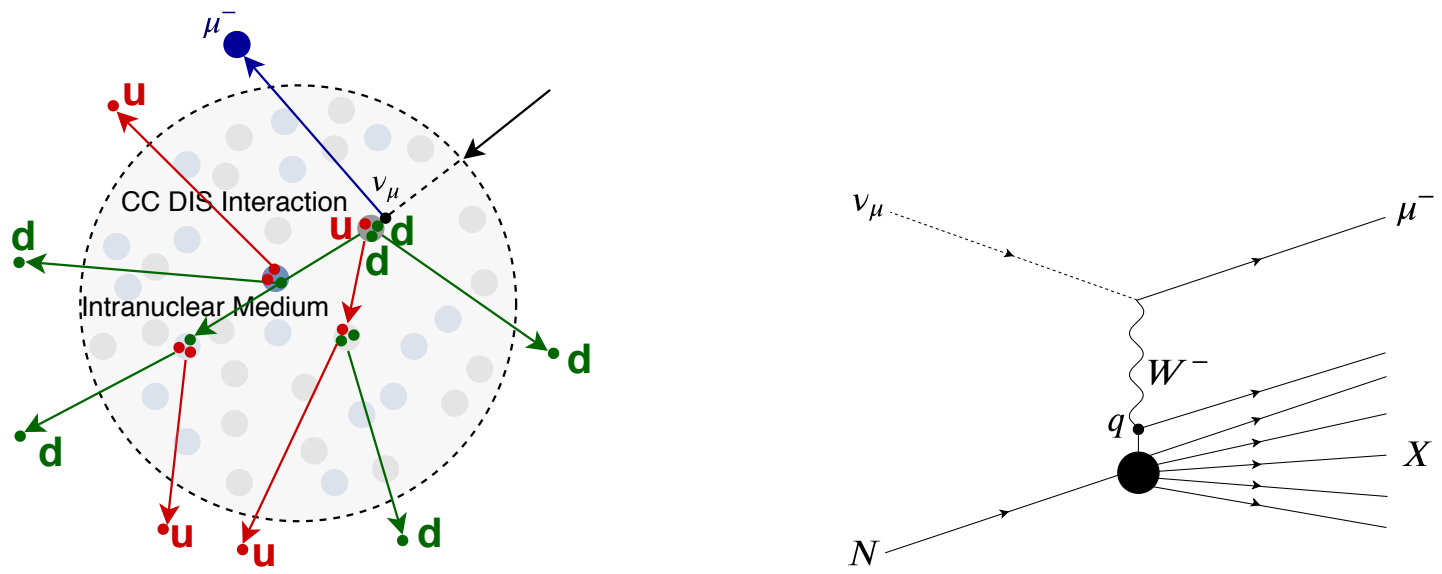

Figure 2.13: (Left) CC DIS scattering on argon, a muon neutrino scatters of a quark inside the struck nucleon, producing a hadronic system. Hadronisation and FSI lead to additional particles exciting the nucleus. (Right) The corresponding Feynman diagram of the process. Figure from [41] 
Chapter 2. Neutrino Physics 


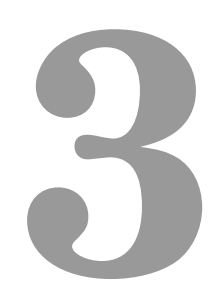

\section{The MicroBooNE Experiment}

The Micro Booster Neutrino Experiment (MicroBooNE) at Fermilab was proposed to resolve the Low Energy Excess (LEE) observed by MiniBooNE. Therefore, it is built $\approx 20 \mathrm{~m}$ away from the MiniBooNE detector, ensuring a very similar $L / E$ value. Furthermore, by sharing the same on-axis beam as neutrino source, the two experiments have the same systematic uncertainties related to the flux simulation. The neutrino beams for MicroBooNE are the topic of Section 3.1. To determine the origin of the LEE, MicroBooNE is equipped with a Liquid Argon (LAr) Time Projection Chamber (TPC), enabling an unprecedented level of energy reconstruction and particle identification. These advantages, alongside with the other detector subsystems of MicroBooNE are discussed in Section 3.2.1. By breaking into uncharted territory as the largest LArTPC deployed in a neutrino beam, MicroBooNE's research \& development results are crucial for the design, commissioning and calibration of 
the next generation neutrino, $0 v \beta \beta$ and dark matter experiments. The fine granularity and high spacial resolution of the technique poses unprecedented challenges to automatisation of event reconstruction, described in Chapter 4. In the last section of this chapter, the main physics motivations of the experiments will be laid out.

\subsection{Neutrino beams at Fermilab}

The use of an artificial neutrino source has two major advantages:

1. The neutrino energy spectrum and its flavour components can be altered by the design of the beam and can be precisely characterised and tuned after commissioning.

2. The position of the detector can be chosen to obtain a more narrow energy spectrum (off-axis) and/or to be located at the expected oscillation peak/dip to enhance the sensitivity of the intended measurement.

The production of the neutrino beam involves a well-defined set of steps and will be described here in the specific case of Fermilab.

Protons are accelerated in the Fermilab LINAC up to $400 \mathrm{MeV}$ kinetic energy. The protons enter the Booster synchrotron where they are further accelerated up to $8 \mathrm{GeV}$ kinetic energy. The Booster has a harmonic number of 84 buckets, of which 81 are filled. The empty ones allow for ramp up/down times for the currents in the kicker magnets.

For the Booster Neutrino Beam (BNB), a fast-rising kicker extracts all the particles in a single turn to transfer them to the target hall. For other purposes, the proton are transferred to the Main Injector. Here, they gain velocity up to the point of $120 \mathrm{GeV}$ kinetic energy. The Main Injector is currently the highest energy accelerator in the USA and is the start point of the Neutrinos from the Main Injector (NuMI), but also the source for the muon experiments at Fermilab and the future high intensity neutrino beam for DUNE. The chain of accelerators and different beams are shown in Figure 3.1 


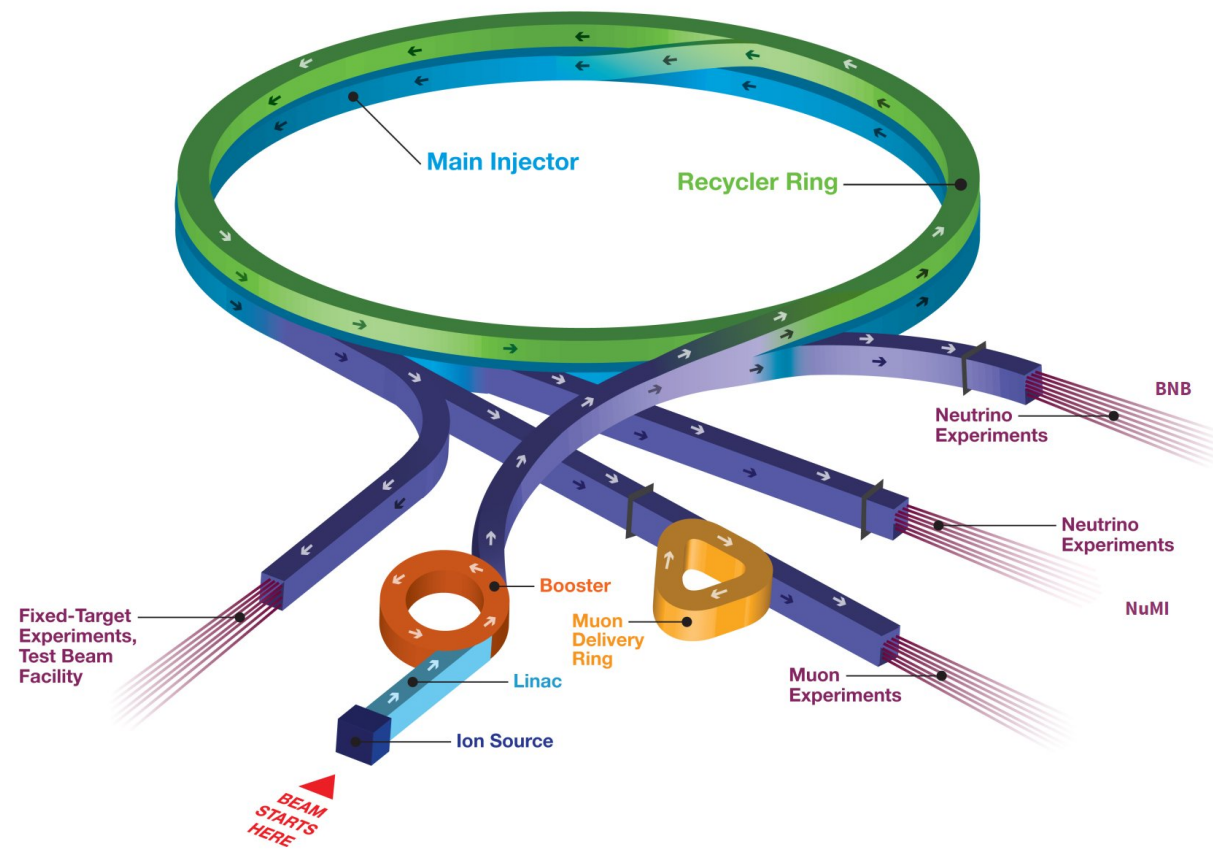

Figure 3.1: The Fermilab accelerator complex. The two beams of interest for the MicroBooNE experiment are BNB and NuMI. Image credit: Fermilab Accelerator Division

\subsubsection{Booster Neutrino Beam (BNB)}

The BNB produces a neutrino beam using the protons from the Booster synchrotron with a momentum of $8.89 \mathrm{GeV} / \mathrm{c}$. The process of beam creation is laid out in Figure 3.2. The proton strike a beryllium target which is embedded in a pulsed electromagnet, the horn. The proton-beryllium interactions lead to the production of mesons such as $\pi^{ \pm}, K^{ \pm}$and $K^{0}$. Of these, the dominant contribution is $p+B e \rightarrow \pi^{+}+X$. When operated in neutrino

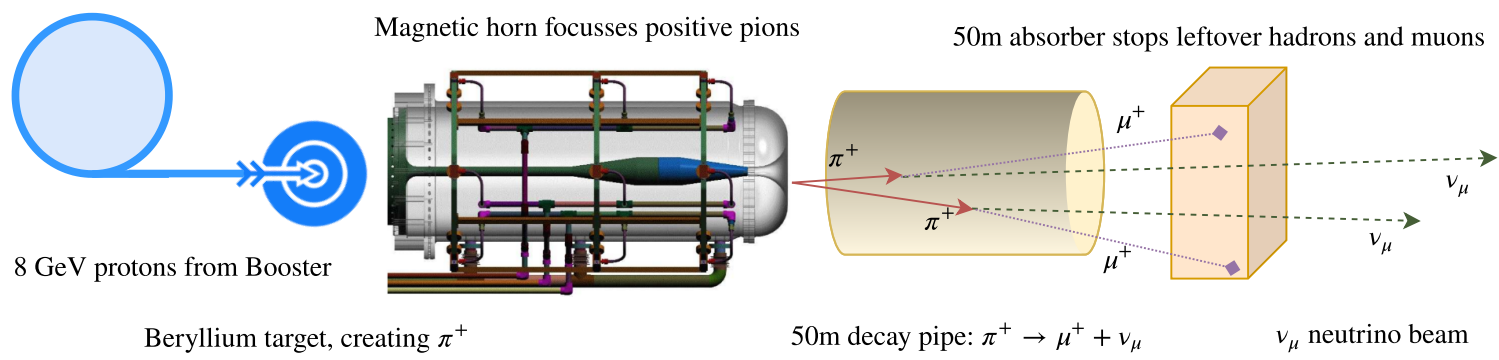

Figure 3.2: Steps in the production of the Booster Neutrino Beam (BNB) in neutrino mode. The main neutrino production mechanism, $v_{\mu}$ 's from $\pi^{+}$decay, is shown. 
mode, the horn will focus the positively charged mesons. The focused secondary beam enters the decay pipe through $\mathrm{a} \approx 2 \mathrm{~m}$ long concrete collimator. In this air-filled cylindrical decay region, a fraction of the constituents of the beam decay, producing neutrinos. The dominant process is $\pi^{+} \rightarrow \mu^{+}+v_{\mu}$. The muons and remaining mesons are then stopped in the beam absorber, which is made out of steel and concrete. The resulting tertiary beam at the end of the beam stop is the neutrino beam. The frequency of the neutrino bunches produced by the $\mathrm{BNB}$ is maximally $5 \mathrm{~Hz}$ and is limited by the design of the horn and its power supply.

Neutrinos can also result from the decay of muons $\left(\mu^{+} \rightarrow e^{+}+v_{e}+\bar{v}_{\mu}\right)$ produced primarily by the decay of pions in the target [43]. This is the main source of the intrinsic $v_{e}$ contamination of the muon neutrino beam, as listed in the third column of Table 3.1. Other production mechanisms for neutrinos lead to further contamination of the $v_{\mu}$ purity of the beam. The dominant source of $\bar{v}_{\mu}$ originates from negatively charged pions that are not filtered out by the horn. Figure 3.3 shows the energy spectrum of the BNB when operated in neutrino mode.

Table 3.1: Predicted neutrino flux at the MicroBooNE detector with the horn in neutrino mode. The composition of the channels is build up from different parent mesons, the two most important ones are given. Adapted from [43]

\begin{tabular}{|c|c|c|c|c|c|c|c|c|}
\hline & \multicolumn{2}{|c|}{$v_{\mu}$} & \multicolumn{2}{|l|}{$\bar{v}_{\mu}$} & \multicolumn{2}{|l|}{$v_{e}$} & \multicolumn{2}{|l|}{$\bar{v}_{e}$} \\
\hline Flux $\left({ }^{\circ} / \mathrm{cm}^{2} / \mathrm{POT}\right)$ & \multicolumn{2}{|c|}{$5.2 \times 10^{-10}$} & \multicolumn{2}{|c|}{$3.3 \times 10^{-11}$} & \multicolumn{2}{|c|}{$2.9 \times 10^{-12}$} & \multicolumn{2}{|c|}{$3.0 \times 10^{-13}$} \\
\hline Fraction & \multicolumn{2}{|c|}{$93.6 \%$} & \multicolumn{2}{|c|}{$5.9 \%$} & \multicolumn{2}{|c|}{$0.52 \%$} & \multicolumn{2}{|c|}{$0.05 \%$} \\
\hline \multirow{3}{*}{ composition } & $\pi^{+}:$ & $96.7 \%$ & $\pi^{-}:$ & $89.7 \%$ & $\pi^{+} \rightarrow \mu^{+}:$ & $51.6 \%$ & $K_{L}^{0}:$ & $70.7 \%$ \\
\hline & $K^{+}:$ & $2.7 \%$ & $\pi^{+} \rightarrow \mu^{+}:$ & $4.5 \%$ & $K^{+}:$ & $37.3 \%$ & $\pi^{-} \rightarrow \mu^{-}:$ & $19.3 \%$ \\
\hline & other: & $0.6 \%$ & other: & $5.8 \%$ & other: & $11.1 \%$ & other: & $10.0 \%$ \\
\hline
\end{tabular}

Figure 3.4 shows the energy dependence of the different parent meson contributions to the total neutrino flux for the $v_{\mu}$ and the $v_{e}$ components. The $K^{+}$contribution is especially important at high neutrino energies and the very lowest neutrino energies. The peak in the $v_{\mu}$ spectrum is due to kaon decay at rest, leading to mono-energetic muon neutrinos of $236 \mathrm{MeV}$. 


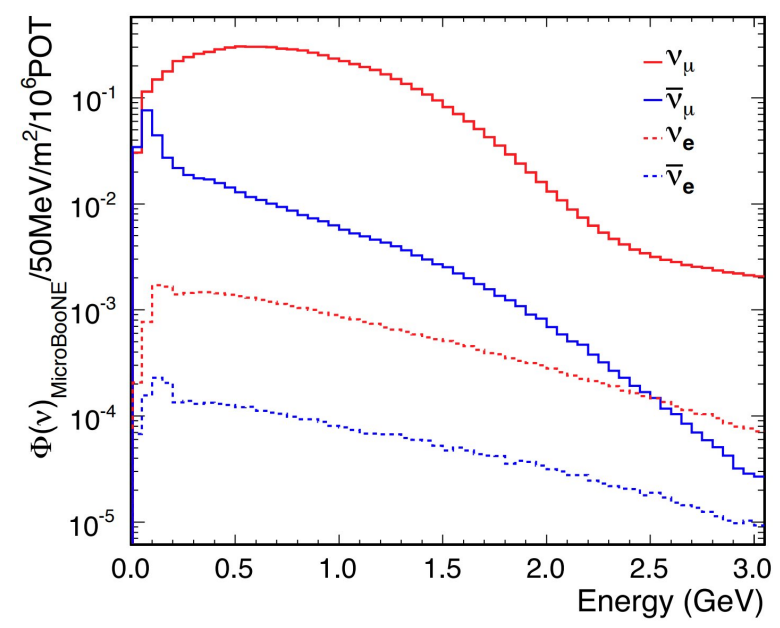

Figure 3.3: BNB energy spectrum in neutrino mode. Figure from [44].

The simulation of the flux by the MicroBooNE collaboration and the source of systematic uncertainties related to the prediction will be discussed in Section 9.1.1.
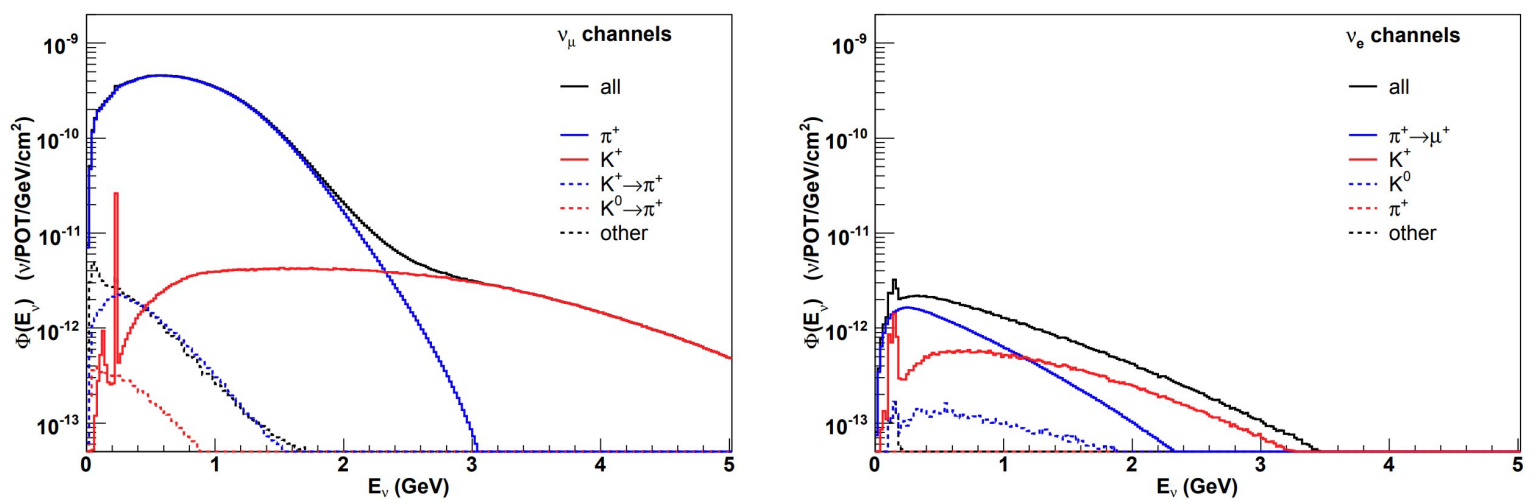

Figure 3.4: Breakdown of parent meson contributions in the BNB for the neutrino modes [43]. The black line is the total predicted flux, while all the sub components apart from the dashed black are from nucleon-induced meson production of the indicated decay chains. The dashed black histogram includes all other contributions, primarily from meson decay chains initiated by meson-nucleus interactions. The double-peaked spectrum arising from the kaon distributions can be split up in decay-in-flight and decay-at-rest. Figure from [43]

\subsubsection{Neutrinos from the Main Injector (NuMI)}

The NuMI neutrino beam was constructed at Fermilab to provide neutrinos for the MINOS experiment, a long-baseline neutrino oscillation search $[45,46]$. Later, the NuMI beam was 
used for other experiments such as Minerva, ArgoNeuT, and most recently the NOvA and the MINOS+ experiments [47, 48, 49, 50,51]. Neutrinos from NuMI have also been observed and studied by the MiniBooNE experiment at a large off-axis angle ${ }^{1}$. The NuMI beam facility produces neutrinos by steering a $120 \mathrm{GeV}$ proton beam onto a narrow graphite target. More information about the NuMI beam can be found in [45].

Since MicroBooNE is located near MiniBooNE, it also receives an off-axis flux from NuMI. Because of MicroBooNE's orientation relative to the NuMI beam dump, it receives a significant amount of low-energy neutrinos from kaon decay at rest. The off-axis component enables MicroBooNE to look at a beam with a narrow energy spectrum and enriched intrinsic electron neutrino contribution. The kaon decay at rest gives rise to a mono energetic muon neutrino beam which can be used for a wide range of validations at low energy.

\footnotetext{
${ }^{1}$ The off-axis location of MicroBooNE with respect to the NuMI beam has the beam entering primarily at $\theta \approx 20$ degrees and $\phi \approx 8$ degrees, with $\theta, \phi$ as in Figure 3.6
} 


\subsection{The MicroBooNE detector}

MicroBooNE is a composite neutrino detector made up out of three detection systems. When charged particles traverse the liquid argon, the argon atoms excite and ionise. The ionisation electrons are collected by the Liquid Argon Time Projection Chamber (LArTPC). This is the main component of MicroBooNE and is discussed in Section 3.2.1. Furthermore, the excited liquid argon atoms produce scintillation light. In MicroBooNE, this is measured by the light system, which consists of 32 PMTs located behind the anode wire planes of the TPC. The scintillation light production and detection methods are described in Section 3.2.2.

Both the Time Projection Chamber and the light system are located inside a cylindrical cryostat filled with $170 \mathrm{t}$ Liquid Argon. Figure 3.5 illustrates the placement of the cryostat inside LArTF in 2014. On the right, the schematic representation shows the inside of LArTF, including the cryostat and surrounding instrumentation. The main components outside the cryostat are the Data Acquisition (DAQ) system, the purification pumps and the high voltage generator.
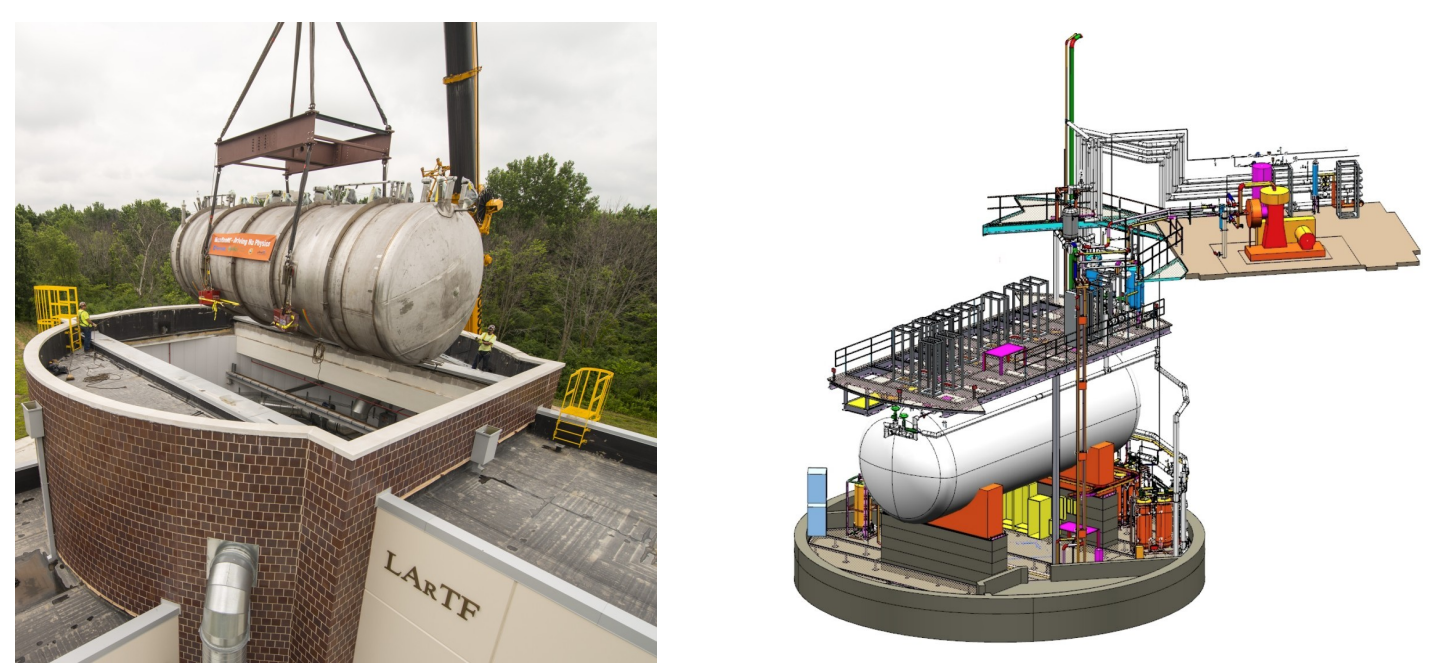

Figure 3.5: (Left) Photo taken during the placement of the MicroBooNE cryostat in LArTF. (Right) Model of the MicroBooNE detector in LArTF. Image from [52].

The upper panels in Figure 3.6 show the MicroBooNE cryostat and the time projection chamber inside it. The readout electronics are inside the liquid argon. The cold environ- 

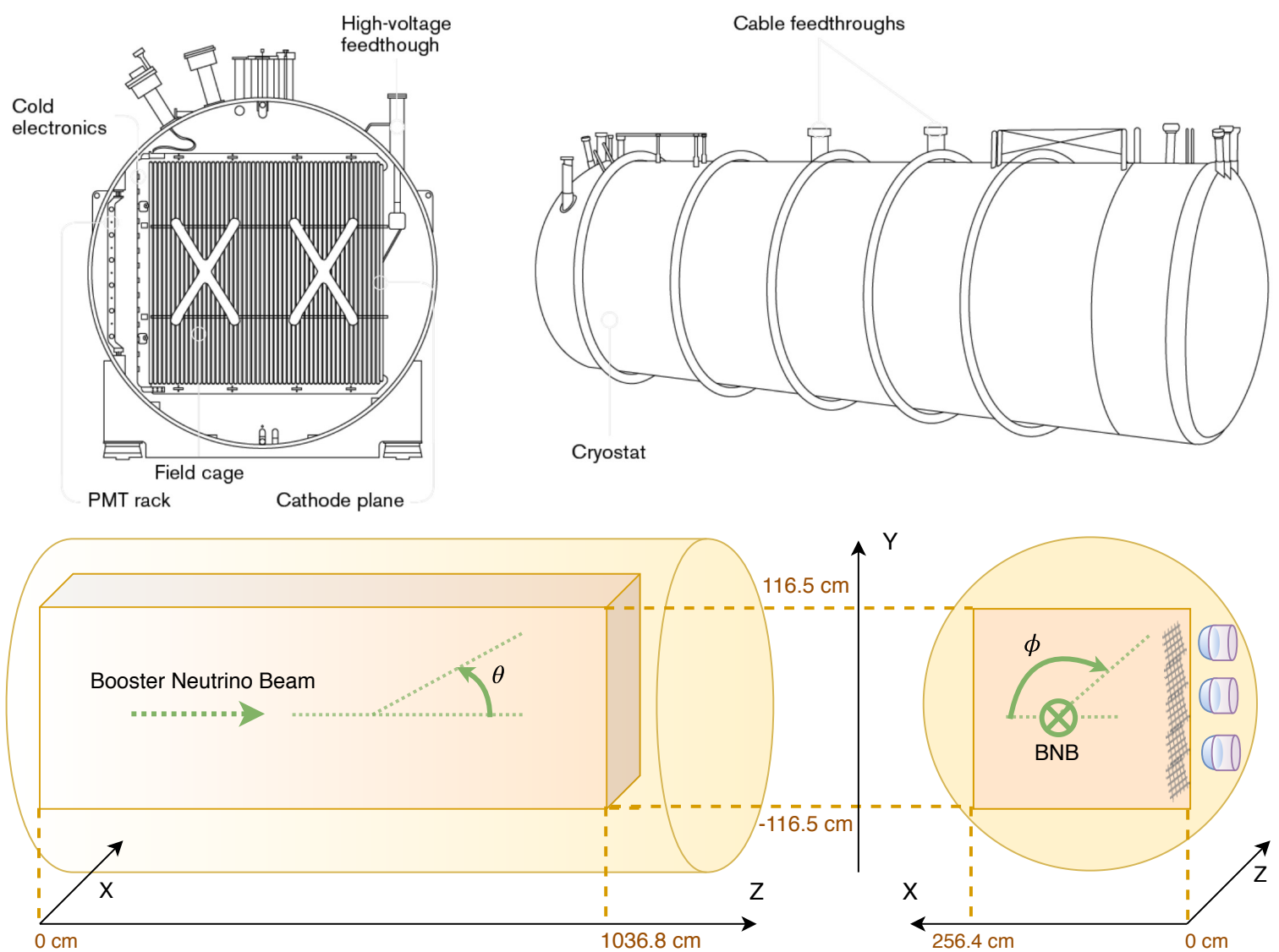

Figure 3.6: (Top) Sketch of the MicroBooNE cryostat. On the left, the TPC field cage is shown, the BNB direction is coming out of the page. Image credit: Symmetry magazine. (Bottom) Definition of the MicroBooNE coordinate system and the $\theta, \phi$ angle of neutrino produced particles. On the right, the beam is going into the page. The wire planes and PMTs are drawn at the anode side

ment substantially reduces the different sources of electronics noise, improving the signalto-noise ratio from the wire signals before amplification. The analogue-to-digital conversion and PMT electronics are outside to the cryogenic environment. The bottom panels illustrate the cuboid TPC and the relative position of the PMTs behind the wire plane. Furthermore, the coordinate frame and dimensions are defined. Due to the $470 \mathrm{~m}$ baseline, the neutrinos travel parallel with the $z$ direction in the MicroBooNE coordinate frame. The direction of particles is defined by two angles; $\theta$ is the angle with the $z$-direction, $\phi$ fixes the orientation in the $X Y$-plane, orthogonal on the beam direction. 
Since 2017, MicroBooNE is equipped with a Cosmic Ray Tagger (CRT) system. This external sub-system is designed to improve the identification and rejection of cosmic muons [53] - MicroBooNE's largest background for neutrino physics - and will be introduced in Section 3.2.3.

\subsubsection{The LArTPC technology}
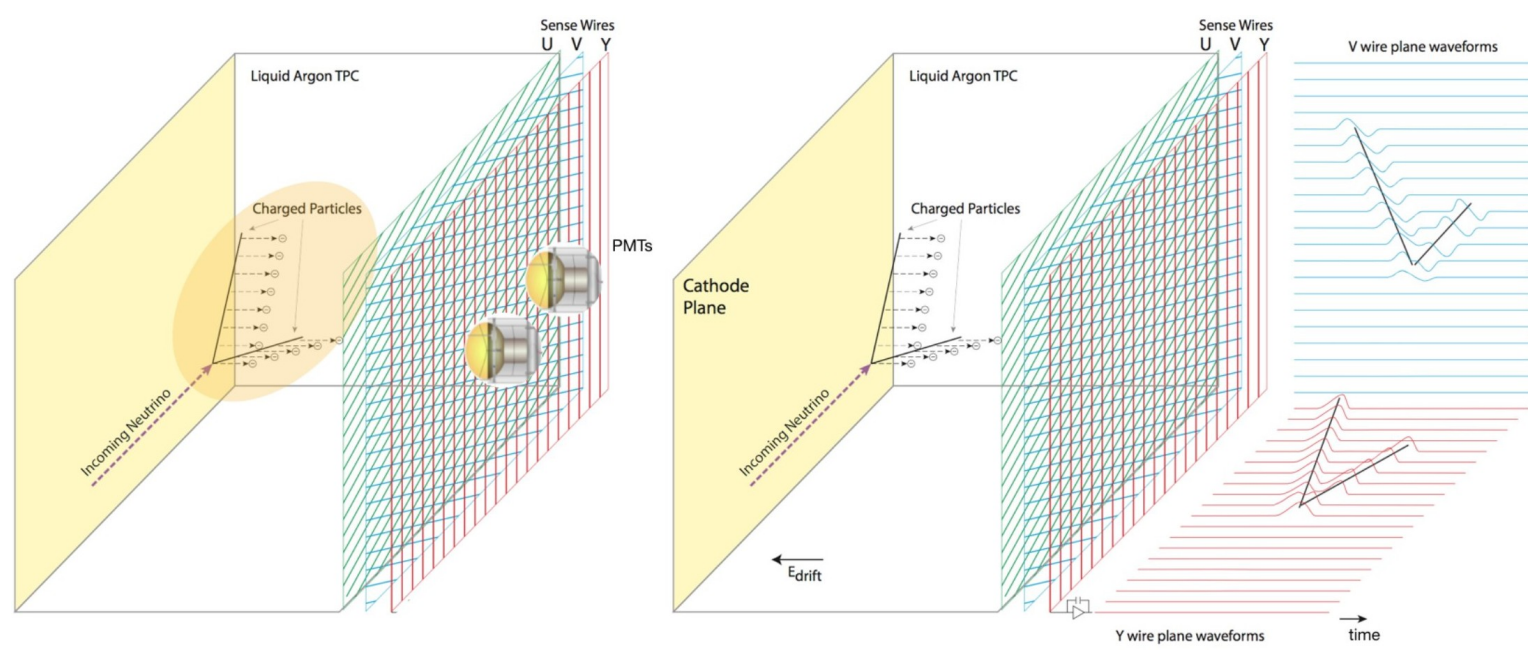

Figure 3.7: Operating principle of the LArTPC neutrino detector. (Left) The fast scintillation light collection by the PMT system. (Right) The slow charge collection by the sensing wires. Figure from [52].

The TPC concept was invented by David Nygren in the late 1970s [54]. In 1977, Carlo Rubbia devised a LArTPC, operating under many of the same principles as Nygren's initial TPC design, but using liquid argon as a sensitive medium instead of gas [55]. Figure 3.7 illustrates the working principles of such a device. The large open cuboid-shaped volume is filled with ultra-pure liquid argon. A homogeneous electric field is created by a high voltage cathode on one side and a grounded anode on the opposite side.

If a neutrino interaction happens inside the volume, it gives rise to charged particles in the final state. These particles excite and ionise the argon as they propagate. Under the influence of a homogeneous electric field, the electrons drift towards the anode plane. To guarantee an $\mathcal{O}(\mathrm{ms})$ electron drift time before recombination, the $\mathrm{O}_{2}$ equivalent contamination should be of the order of 10 parts per trillion. At the anode, three planes of sensing 
wires are spun. The clouds of electrons create signals on the wires, as illustrated in the right panel of Figure 3.8. The first two planes are oriented $\pm 60^{\circ}$ relative to the vertical axis. To generate multi-dimensional views of particle tracks, at least two planes at different orientations are needed. A third plane reduces ambiguities originating from unresponsive wires, and improves calorimetry and tracking capabilities, especially for trajectories parallel with one of the planes. Bias voltages are applied to each wire plane to ensure the two induction planes satisfy the transparency condition that all drifting electrons pass by the induction plane wires and fully collect on wires in the last (collection) plane [56,57]. The charge drifts past the first two planes, inducing a bipolar signal and is collected by the third plane, producing a unipolar signal proportional to the total ionisation in that location.

For MicroBooNE specifically, the dimensions of the TPC field cage are $2.3 \mathrm{~m}$ ( $y$, height $) \times$ $2.6 \mathrm{~m}(x$, width $) \times 10.4 \mathrm{~m}(z$, length, along the beam direction), elucidated by Figure 3.6. The TPC is submerged in liquid argon, which is kept at a pressure of $1.2 \mathrm{~atm}$ and a boiling temperature of $89 \mathrm{~K}$, leading to a density of $1.38 \mathrm{~g} / \mathrm{cm}^{3}$. The active mass inside the TPC is $86 \mathrm{t}$. The homogeneous electric field is generated by biasing the cathode at $-70 \mathrm{kV}$, while keeping the anode grounded. The electric field of $273 \mathrm{~V} \mathrm{~cm}^{-1}$ corresponds to an electron drift velocity of $114 \mathrm{~cm} / \mathrm{ms}$. Therefore, reading out the charge corresponding to one snapshot requires $2.3 \mathrm{~ms}$. The transparency conditions of the induction planes are achieved by biasing the $U, V$ and $Y$ planes at respectively $-110 \mathrm{~V}, 0 \mathrm{~V}$, and $230 \mathrm{~V}$. Each of the induction planes $U$ and $V$ consists of 2400 wires, while the collection plane $Y$ has 3456 wires. The inter-plane distance is $3 \mathrm{~mm}$ and the distance between the wires on the same plane is also $3 \mathrm{~mm}$.

The analogue sense wires signals are passed through an front end ASIC in the cold liquid argon environment. The signals are then shaped and amplified by cold intermediate amplifiers before passing through feed-through. The next steps of the signal processing happen outside of the cryogenic environment. The signals are received by custom-designed readout modules, where they are digitised and processed. The sampling frequency is $16 \mathrm{MHz}$ and the signals are then down-sampled in the digitisation process to $2 \mathrm{MHz}$ (500 ns time-ticks). 

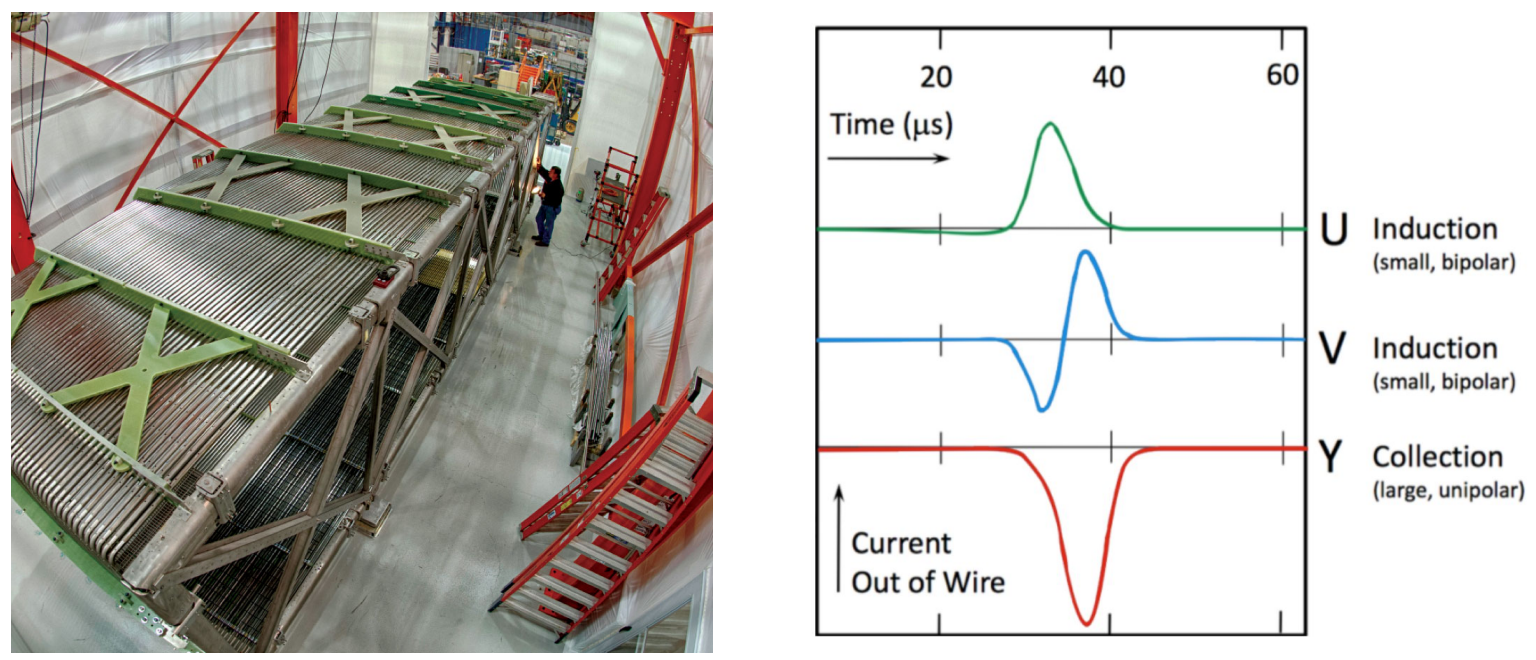

Figure 3.8: (Left) Picture of the MicroBooNE TPC before installation in the cryostat. (Right) Example of the waveforms recorded by the different wire planes in MicroBooNE for a charge deposit in the TPC. Figure from [52].

The TPC readout window consists of three $1.6 \mathrm{~ms}$ frames of wire signal data associated with one event. Further downstream this is truncated to an interval from $-0.4 \mathrm{~m} \mathrm{~s}$ to $2.7 \mathrm{~m} \mathrm{~s}$, the 0 is defined by the hardware trigger received from the accelerator division. This duration is chosen based on how long it takes for ionisation electrons from the cathode side of the TPC to drift to the anode wires, in the case of the MicroBooNE, $2.3 \mathrm{~ms}$. The additional $0.4 \mathrm{~ms}$ buffer on either side ensures enough data to identify the neutrino interaction, as well as all cosmic activity that arrives close enough before or after the neutrino beam trigger.

An excerpt of the collection plane view of a neutrino interaction is given in Figure 3.9. In the collection plane, the beam direction is perpendicular to the wires. The vertical axis should be interpreted the electron drift time, defined as the $x$-direction in the TPC. With the collection plane only, no information can be interfered of the TPC $y$-direction. A typical neutrino event will span from $\mathcal{O}(15 \mathrm{~cm})$ at the lowest energies $(\mathcal{O}(500 \mathrm{MeV}))$ and several meters for high-energy Minimal Ionising Particle (MIP) tracks.

The argon ion drift velocity, approximately $5 \mathrm{~mm} / \mathrm{s}$, is more than six orders of magnitude smaller than the electron drift velocity. Therefore the build-up of positive charge by argon ions inside the TPC lasts of the order of minutes. Furthermore, as will be the topic 
of Chapter 5 , the cosmic muon bombardment at a rate of $\approx 5 \mathrm{kHz}$ through the TPC creates a continuous source of positively charged drifting argon ions. This presence of positive charge can be approximated as a steady-state distortion of the homogeneous electric field inside the TPC. This distortion is referred to as space charge effect and mimics a displacement in the reconstructed position of signal ionisation electrons of up to $\mathcal{O}(10 \mathrm{~cm})$. The space charge effect is discussed in more detail in Appendix B.

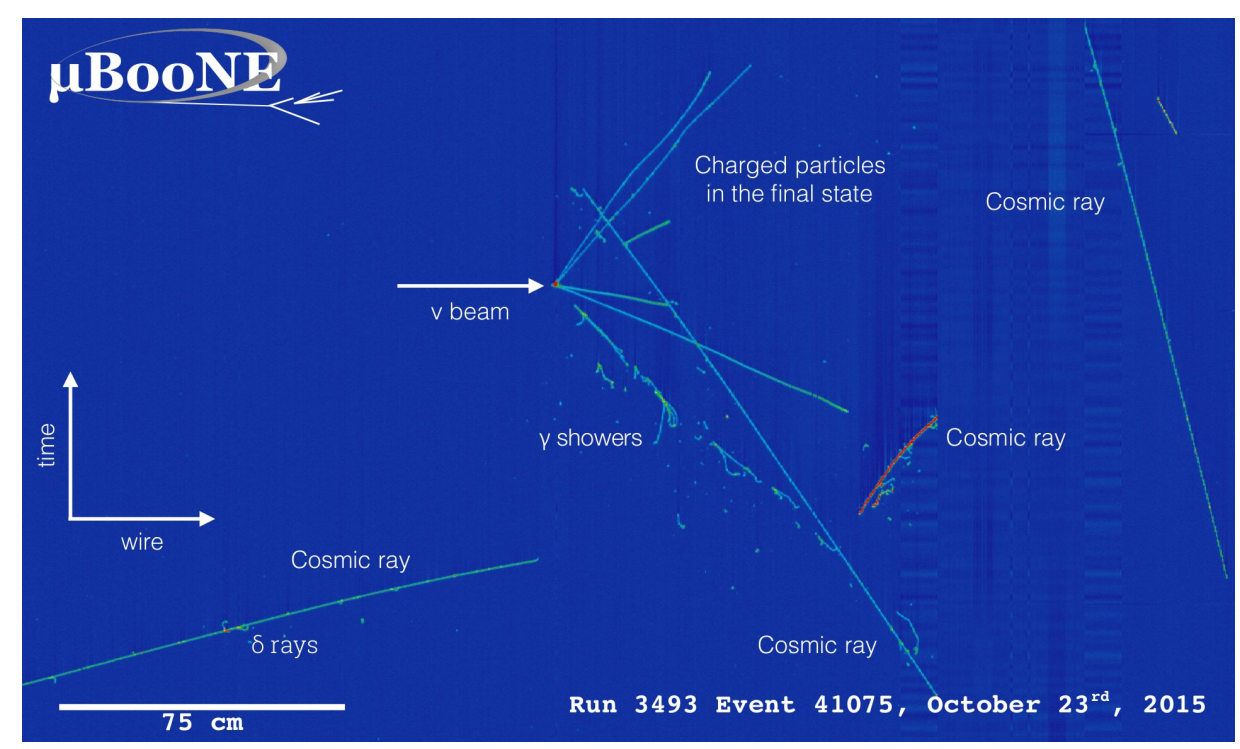

Figure 3.9: Event display of a $v_{\mu} \mathrm{CC} \pi^{0}$ candidate as seen on the collection plane. The colour scale corresponds to the amount of charged deposited on the wires. Figure from [58].

\subsubsection{Light production \& collection in MicroBooNE}

Compared to the slow drifting $-\mathcal{O}(\mathrm{ms})-$ TPC signal, the production and propagation of scintillation light is instantaneous $(\mathcal{O}(n s))$. Therefore, collecting scintillation light is essential to provide the time of the event and therefore resolve the $x$-position in the TPC drift direction. Section 6.2 will demonstrate how a neutrino selection can further benefit from exploiting the scintillation light information. In this section, we will first discuss the properties of scintillation light in liquid argon, and then the light system employed by MicroBooNE for its detection. 


\section{Scintillation light in liquid argon}

When charged particles travel through LAr, scintillation light is produced with a yield of tens of thousands of photons per deposited $\mathrm{MeV}$. This section aims to elucidate on the production mechanism and properties of scintillation light in liquid argon. Figure 3.10 illustrates the two chains through which light can be produced:

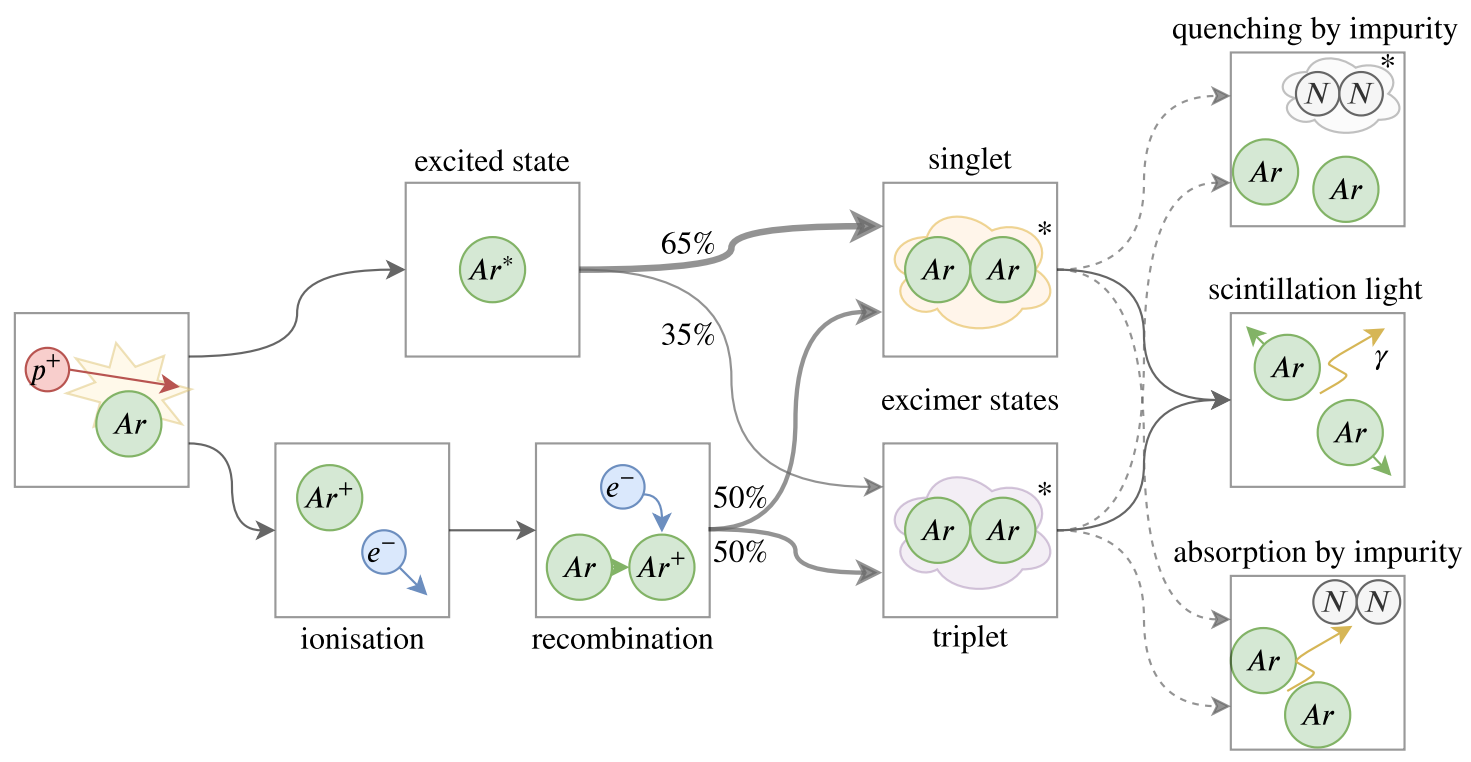

Figure 3.10: Scintillation light production in Liquid Argon. A charged particle excites (upper sequence) or ionises (lower sequence) the argon atoms. The first process gives rise to selftrapped exciton luminescence while the latter can lead to recombination luminescence. In both of these mechanisms, the scintillation light production requires the formation of excimers. The production of scintillation light can be suppressed through quenching or absorption by impurities, such as nitrogen, as shown on the panels on the right.

\section{Self-trapped exciton luminescence}

Charged particles travelling in the LAr leave a fraction of the argon atoms in excited states. these excitons form a short-lived dimeric molecule with another argon atom, called dimers or excimers. The majority of those are in the singlet state ${ }^{1} \Sigma_{u}$, and approximately one-third correspond to the triplet state ${ }^{3} \Sigma_{u}$.

2. Recombination luminescence

Alternatively, the charged particles can ionise the argon atoms,creating free electrons. When free electrons recombine with the positive Ar atoms, these can again create 
excited dimers. In this process the creation of the singlet or triplet excimer states are equally likely.

The ${ }^{1} \Sigma_{u}$ singlet gives rise to a fast scintillation light component with a decay time of $\approx 6 \mathrm{~ns}$ while the ${ }^{3} \Sigma_{u}$ triplet has a decay time of $\approx 1500 \mathrm{~ns}$. The scintillation light in liquid argon will therefore consist of well separated fast and slow components, both narrowly peaked in the Vacuum Ultra-Violet (VUV) region at $128 \mathrm{~nm}$. Both of the excimer states have an energy minimum corresponding to inter-atom distance of approximately $2.8 \AA$ (see Figure 3.11 ). Argon in its liquid state has a separation of $\approx 4 \AA$, well separated from the excimer minima. Therefore it is unlikely for liquid argon at nominal pressure to absorb a significant fraction of its own scintillation light. This transparency of liquid argon to its own scintillation light allows for detection over long distances with minimal attenuation.

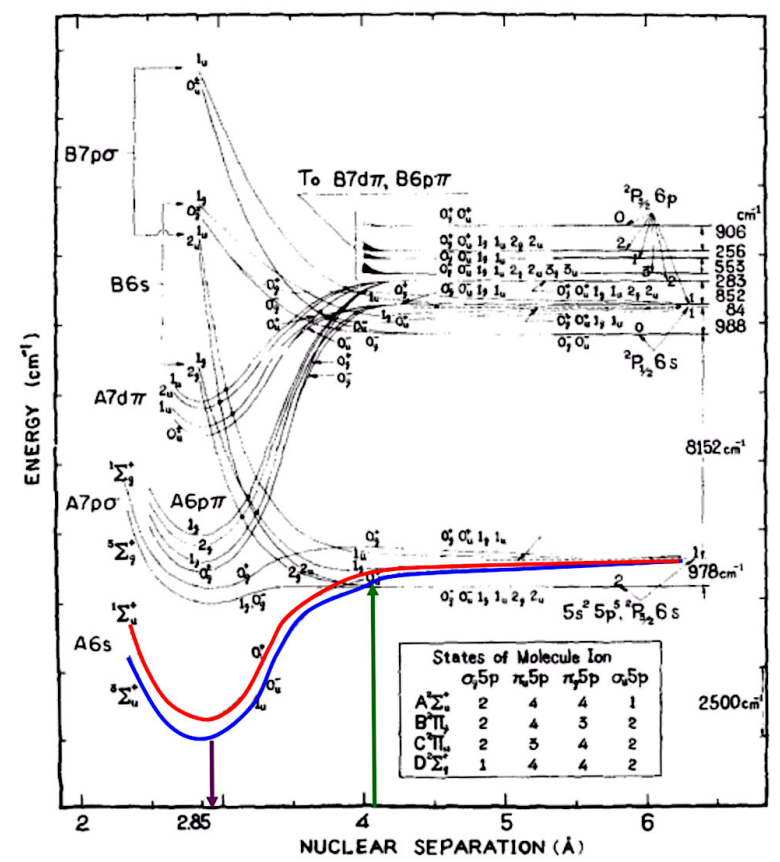

Figure 3.11: Excimer state energies as a function of nuclear separation. The two states of interest for the scintillation light are indicated in red (singlet, fast component) and blue (triplet, slow component). Adapted from [59].

The process of recombination luminiscence depends linearly on the local density of both the ionised argon atoms and the free electrons. Recombination is therefore enhanced for highly ionizing particles (high $\mathrm{d} E / \mathrm{d} x$ ). This introduces a dependence on the deposited energy to 
light conversion on the particle type, which will be discussed in Section 6.3.2. The same reasoning also explains how the scintillation yield in liquid argon depends on the strength of the electric field. A stronger electric field will increasingly pull the positive argon items and free electrons away from each other, lowering the production of scintillation light through recombination luminiscence.

As illustrated in the right panels of Figure 3.10, impurities can reduce the amount of scintillation light through quenching and absorption. In MicroBooNE, the purity of the LAr is monitored to assure the absorption length stays well above the drift width of the TPC (2.5 m). Nevertheless during the data-taking of MicroBooNE between May 2016 and May 2019, a decrease of the light yield was observed, shown in Figure 3.12. This decrease is partially attributed to the increase of impurities by a refill of LAr.
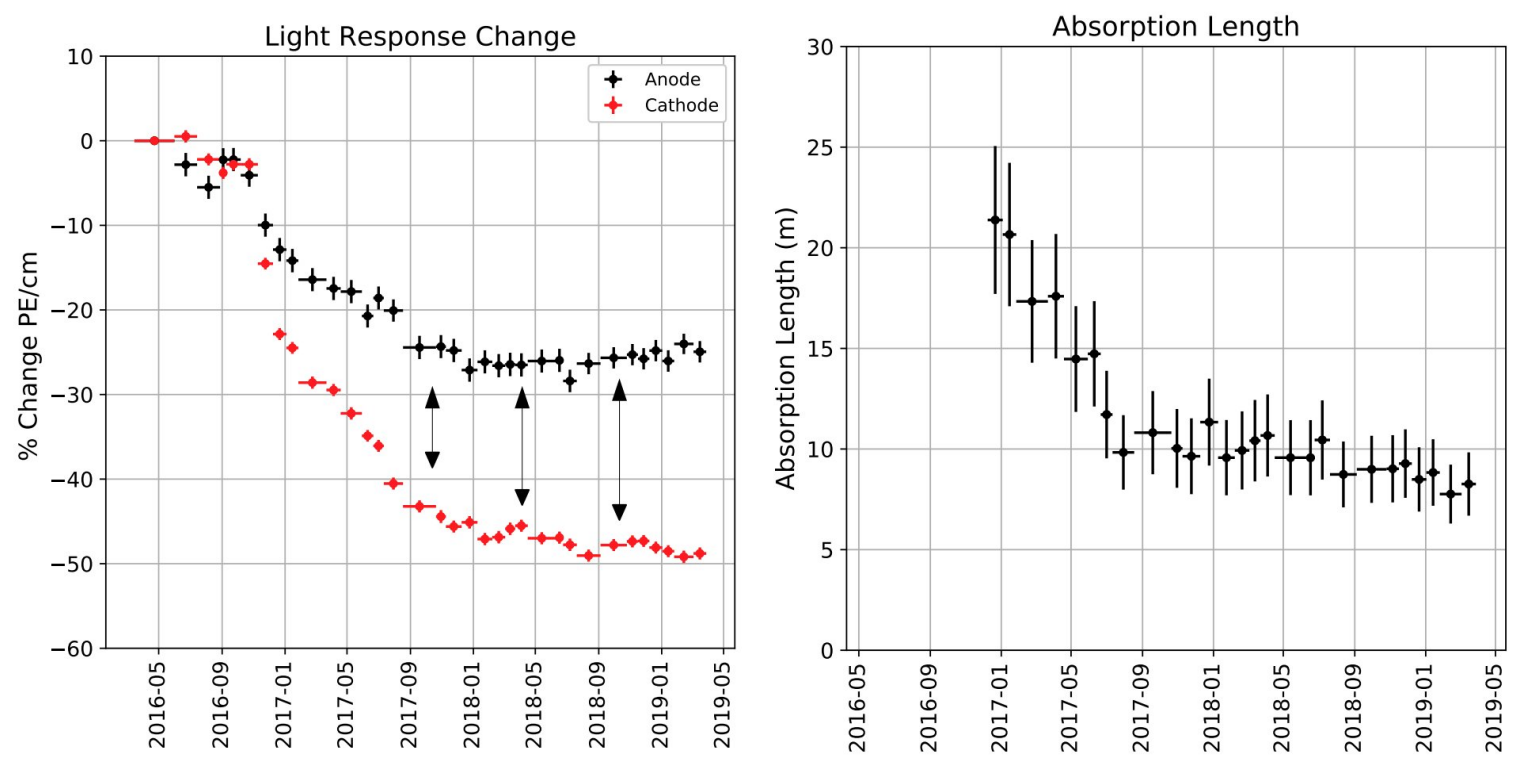

Figure 3.12: Percentage decrease of light yield in MicroBooNE, measured as the reduces number of photo-electrons per muon track-length, $\mathrm{PE} / \mathrm{cm}$. The decrease in light yield is interpreted as a decrease in the absorption length by impurities. (Left) the decrease is split up near the anode, close to the PMTs and the cathode. (Right) The difference at the anode and the cathode can be interpreted as a decrease of the absorption length over time. Figure courtesy of Patrick Green. 

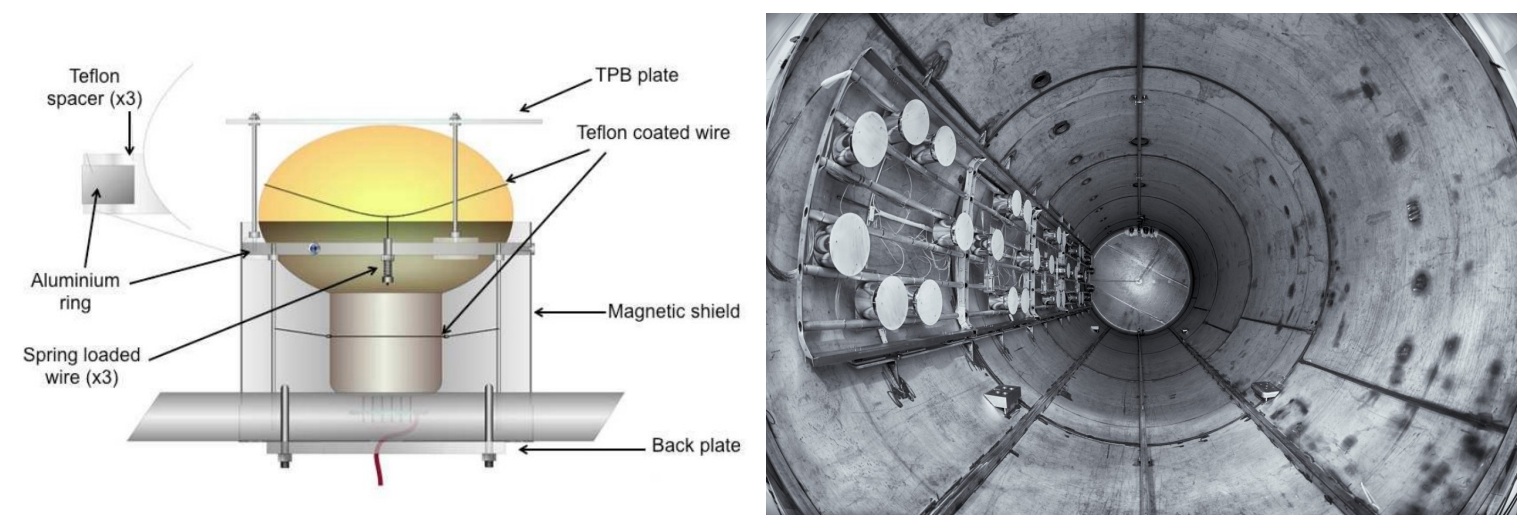

Figure 3.13: (Left) A schematic drawing of the PMT mount with a PMT. A wire ring is pulled down by 3 spring loaded wires to an aluminium ring. Direct contact of the PMT to the aluminium ring is avoided by Teflon blocks. The magnetic shield and the TPB plate are fixed on to the PMT mount. (Right) Picture showing the 32 PMTs attached to the side of the MicroBooNE cryostat. Figures from [60].

\section{The light collection system}

The left panel of Figure 3.13 shows the basic building block of MicroBooNE's light detection system: the 8 inch diameter Hamamatsu R5912-02mod cryogenic Photo-Multiplier Tube (PMT). In front of the PMT, a Tetra-Phenyl Butadiene (TPB) coated acrylic plate is mounted. The coating is essential to convert the $128 \mathrm{~nm}$ LAr scintillation light to the visible spectrum, peaking at $425 \mathrm{~nm}$, where the quantum efficiency of the device peaks (see Figure 3.14). Furthermore, the tube is surrounded by a magnetic shield made out of mu-metal to shield the sensitive electronic components against static or low-frequency magnetic fields.

The optical sensors are situated in the $Y Z$ plane in detector coordinates, mounted on a frame behind the TPC wire planes, as can be seen in the right photograph of Figure 3.13. The operational voltage of the PMTs is $1300 \mathrm{~V}$, corresponding to an electronic gain of $\approx$ 10.7 [63]. The analogue PMT signal is recorded and passed through a splitter board in a high- and low-gain channel, of $18 \%$ and $1.8 \%$ respectively. The splitter increases the effective dynamic range of the amplifying circuit by picking the appropriate data-stream depending on the saturation or weakness of the signal. Both signals are pre-amplified and shaped before being digitised at $64 \mathrm{MHz}$. After the shaper, the signals have a rise time of $60 \mathrm{~ns}$, negligible compared to the $1.6 \mu$ s or $10 \mu$ s spill windows of respectively the BNB and 


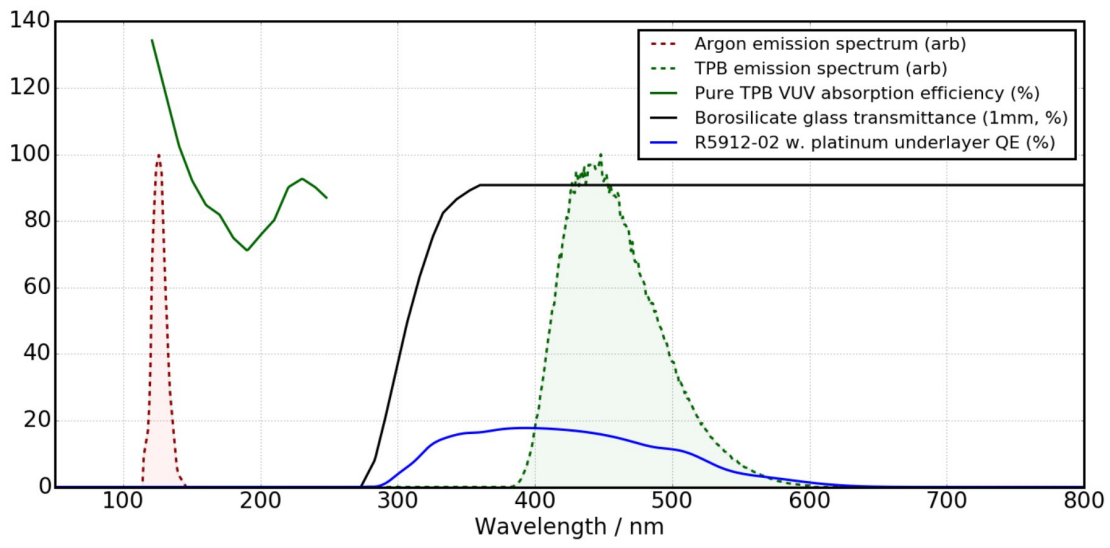

Figure 3.14: Scintillation spectrum of argon; Emission spectrum of TPB; VUV absorption efficiency of TPB; borosilicate glass transmittance; Hamamatsu bialkali photocathode quantum efficiency; and platinum undercoated PMT quantum efficiency from data provided by Hamamatsu for MicroBooNE PMTs [61]. The absorption, as defined in here, can exceed $100 \%$ if multiple photons are emitted per absorbed photon. Figure from [62]

NuMI beams.

\subsubsection{The cosmic ray tagger system}

The Cosmic Ray Tagger (CRT) system is an external sub-detector and was installed after one year of data-taking in July-September 2016 (Phase I) and February 2017 (Phase II). The system consists of 73 scintillating modules made of interleaved layers of scintillating plastic strips situated on the top, bottom, and two sides parallel to the neutrino beam (Figure 3.15, left panel) [53]. Using a combination of COsmic Ray SImulations for KAscade (CORSIKA) [64] and GEometry ANd Tracking: A platform for the simulation of the passage of particles through matter (GEANT) [65], the coverage is estimated to be $85 \%$ (Figure 3.15 , right panel).

The main objective of the CRT is to improve the identification and rejection of cosmic activity. Scintillation light induced by cosmic muons passing through CRT planes can be reconstructed as hits on specific channels. These hits enable the determination of the time of TPC-crossing-muons with a precision of $\mathcal{O}(100 \mathrm{~ns})$, hereby enabling another mechanism (appart of the PMT system) to overcome the ambiguity along the $x$-direction in the TPC 


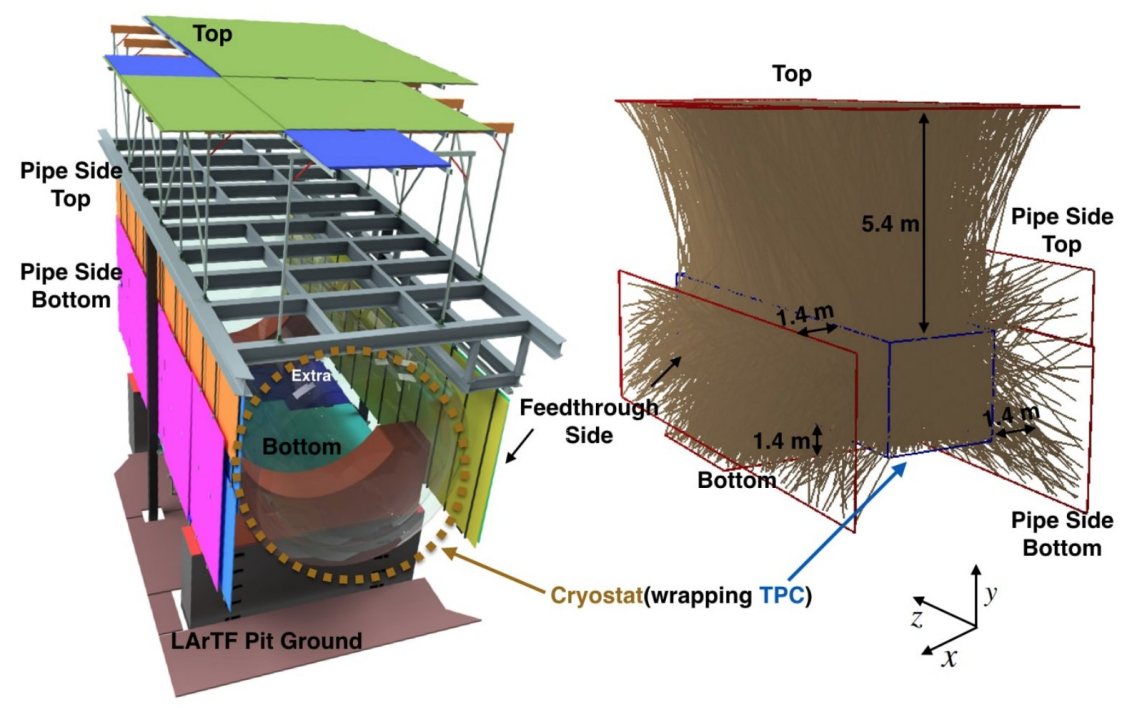

Figure 3.15: (Left) The design of the Cosmic Ray Tagger planes as part of the MicroBooNE detector. There are four Cosmic Ray Tagger planes: top plane, bottom plane, pipe side plane and feed-through side plane. The beam direction is along the $z$-axis. (Right) Simulation of cosmic rays crossing the Cosmic Ray Tagger, the brown lines represent possible cosmic ray trajectories. Figure from [53]

reconstruction. In Section 5.4, the system is used to measure the cosmic backgrounds and compared with simulation. In Chapter 6, a set of tools to enhance the cosmic rejection for neutrino studies relying on CRT information will be discussed. 


\subsection{Physics motivations}

The MicroBooNE experiment is the largest LArTPC for neutrino detection ever commissioned and the first experiment to have completely automatic event reconstruction of LArTPC data. It is therefore able to access a plethora of new physics measurements. Apart from being a crucial step in the research \& development towards the next phases in the United States' LArTPC programme, it covers a wide range of physics topics. The main physics goals will briefly be introduced in this section and include: validation of short-baseline anomalies through electron neutrino appearance, precision cross-section measurements on argon using muon neutrinos, measurements of nuclear effects using exclusive channels, astro-particle physics and exotics.

\subsubsection{Validation of short-baseline anomalies}

The primary goal of the MicroBooNE experiment is to investigate the MiniBooNE Low Energy Excess (LEE), as introduced in Section 2.4.1. By running with the same beam and a very similar baseline, MicroBooNE is designed to confirm or refute the excess of electromagnetic events as was observed by MiniBooNE [23]. Furthermore, the LArTPC technology enables to differentiate between electrons and photons and therefore provide information about the underlying physics producing the excess.

Several proposals have been made to explain the nature of the MiniBooNE LEE. It is fair to say that a large amount of uncertainty remains in the community regarding what may have generated such an excess of electromagnetic events: Chapters 8 and 9 will focus on the search of an electron neutrino excess.

\subsubsection{Cross section measurements \& study of nuclear effects}

MicroBooNE collected the largest amount of neutrino interactions on liquid argon to date. At the BNB energy regime, $\mathcal{O}(\mathrm{GeV})$, a wide variety of the effects discussed in Section 2.5 can be studied. One ongoing effort investigates the use of single transverse variables (STV) to characterise the transverse imbalance and therefore nuclear effects directly [41]. The con- 
tributions from different nuclear interaction types to the charged-current neutrino interactions in both muon and electron flavour are shown in Figure 3.16. The neutrino selection and subsequent muon identification that are the foundation of these searches will be the topic of Chapters 6 and 7.
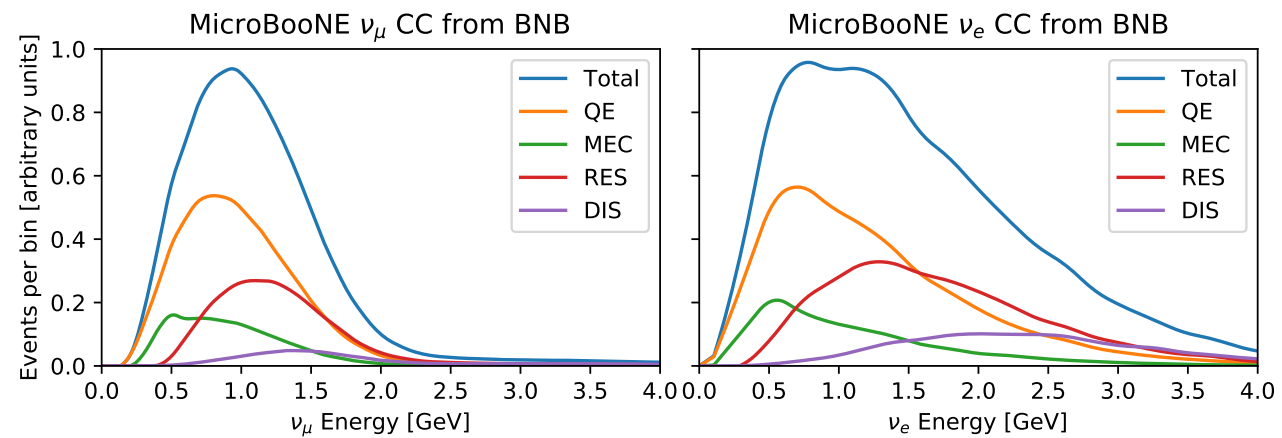

Figure 3.16: Breakdown of neutrino interactions in MicroBooNE by different nuclear interaction types.

For the purpose of the electron neutrino analysis, measurements of $v_{\mu}$ interactions are aimed at reducing modelling uncertainties for intrinsic $v_{e}$ events. Constraining backgrounds and systematic uncertainties is a crucial step to hypothetically claim the observation of new physics if a measurement of $v_{e}$ interactions differs significantly from the expected intrinsic rate. Chapter 9 describes such a data-driven constraint.

\subsubsection{Astro-particle physics \& exotic physics}

MicroBooNE is able to pioneer physics results in a variety of diverse neutrino and nonneutrino physics. Some examples are:

- Searching for heavy non-relativistic sterile neutrino states [66].

- The ability to detect supernovae with LArTPC technology [67].

- Study of Reconstructed ${ }^{39}$ Ar Beta Decays [68].

While the first is interesting in its own right, the latter two are important for the low-energy physics programme at DUNE. 
Chapter 5 characterises the cosmic-muon flux in the different MicroBooNE sub-detection systems. This work is essential to better understand and characterise our dominant background, cosmic activity. Furthermore it can be expanded to include additional precision studies, fine-tuning the simulation of cosmic activity at Fermilab.

Finally, as the forerunner to future LArTPC experiments, MicroBooNE provides a unique environment for technology development. Among other topics, improvements in highvoltage distribution, light collection, LArTPC reconstruction methods and calibration techniques are essential for the next-generation liquid argon experiments. 


\subsection{Beyond MicroBooNE: the Short-Baseline Neutrino Programme}

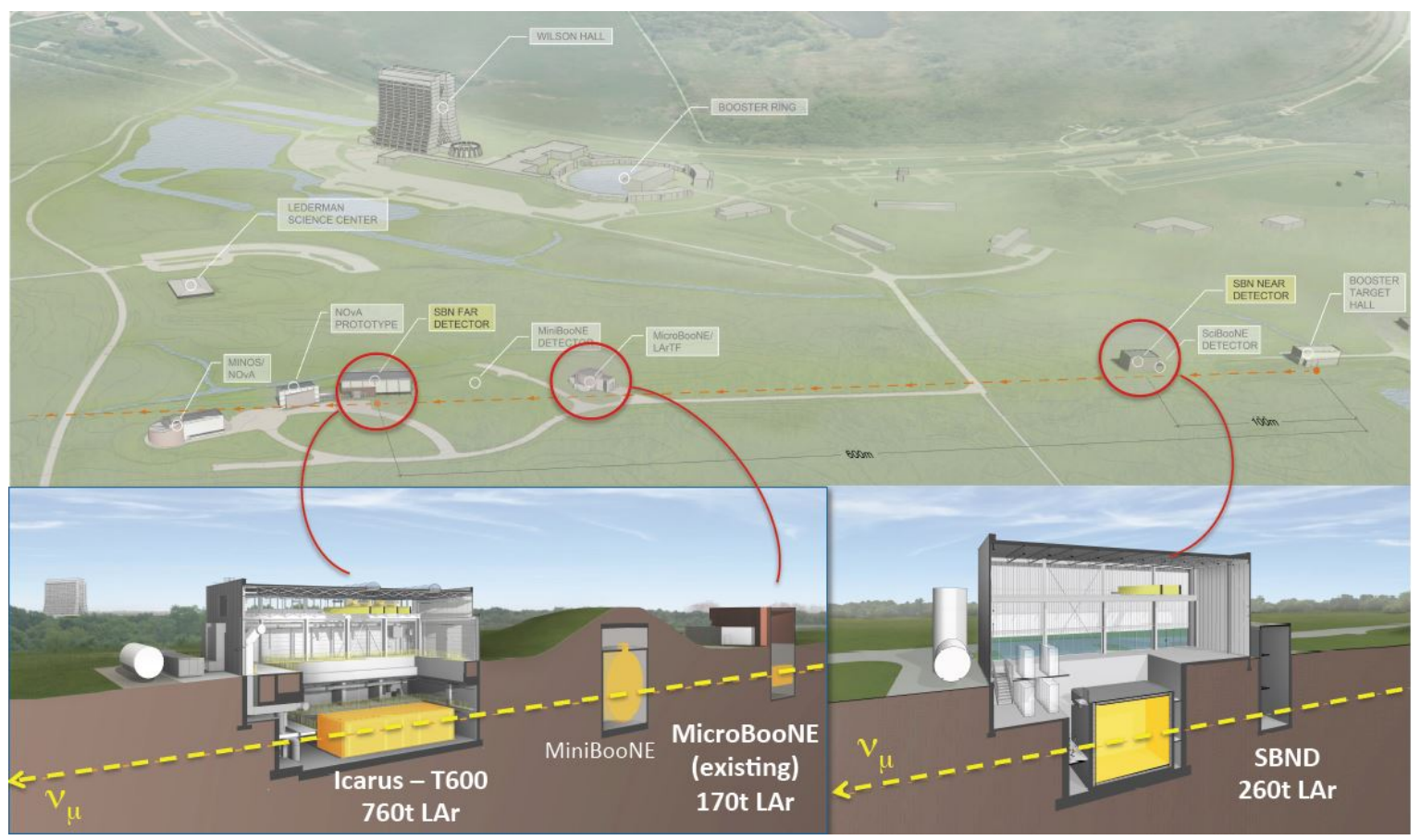

Figure 3.17: An aerial view of the Short-Baseline Neutrino experimental area at Fermilab. To the right is the neutrino beam target area where $8 \mathrm{GeV}$ protons from the Booster accelerator impinge a beryllium target. The beam is focused along the orange dashed line (approximately $7 \mathrm{~m}$ below grade) traveling toward the left (north). The Near Detector, MicroBooNE, and Far Detector building locations are indicated. Image credit: Holabird \& Root.

MicroBooNE serves as the first phase in the LArTPC Short-Baseline Neutrino Program at Fermilab (SBN). This project, consisting of three detectors employing the same beam and technology but placed at different baselines, is designed for a full oscillation search in parameter region of interest for the short-baseline anomalies introduced in the previous chapter. Figure 3.17 serves as an overview of the project. The near-target detector is called SBND and serves a double purpose. On one side, the high flux near the neutrino source enable to do a wide variety of exclusive neutrino cross-section measurements, important to improve our current knowledge about neutrino-argon scattering. Secondly, as a near detector for the two other TPCs, it enables a strong constraint on the flux systematic uncertainties. SBND is expected to start data-taking in 2021. 
The far detector, called ICARUS, is filled with $760 \mathrm{t}$ of liquid argon at a distance of $600 \mathrm{~m}$ from the target. ICARUS started operations in March 2020 and will soon be able to see its first neutrino events.

In the next few years, the SBN programme will provide powerful new input to the question of light sterile neutrinos. Figure 3.18 shows the projected sensitivities to the $3+1$ sterile neutrino hypothesis of the combined near and far analyses with $6.6 \times 10^{20} \mathrm{POT}$, corresponding to approximately 3 years of data-taking in neutrino mode with the BNB. The experiment's ability to simultaneously observe $v_{e}$ appearance and $v_{\mu}$ disappearance oscillation channels is a key ingredient to solving the light sterile neutrino puzzle.
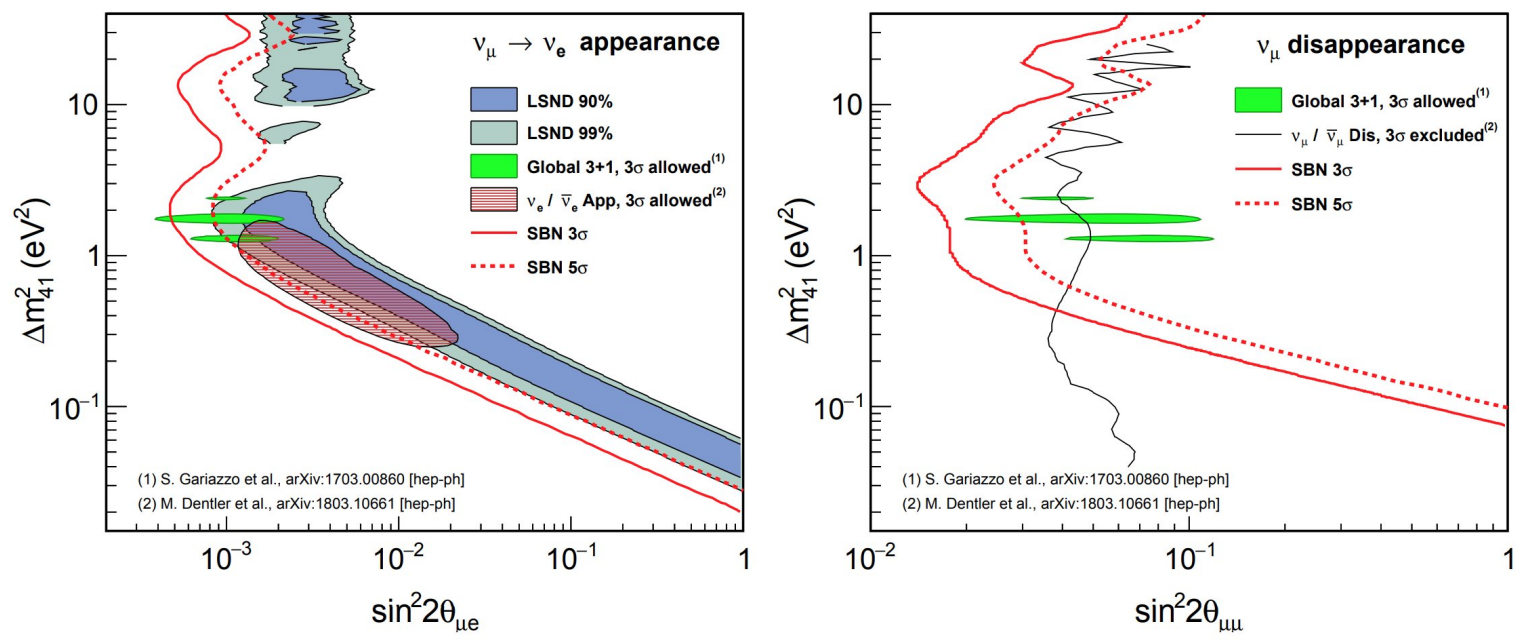

Figure 3.18: SBN 3 (solid red line) and 5 (dotted red line) sensitivities to a light sterile neutrino in the $v_{\mu} \rightarrow v_{e}$ appearance channel (left) and $v_{\mu} \rightarrow v_{\mu}$ disappearance channel (right). For comparison, the LSND preferred region at 90\% C.L. (shaded blue) and $99 \%$ C.L. (shaded gray) is presented. Moreover, the global $v_{e}$ appearance (shaded red) and global $v_{\mu}$ disappearance (black line) 3 œ regions are also included. Finally, the $3 œ$ global best fit regions are shown in green. The sensitivities are taken from the SBN proposal. Figure from [69].

Figure 3.19 illustrates how a sterile neutrino with a certain mass and mixing strength might manifestate in two plausible scenarios. From Equation (2.18), we find that the oscillation maximum is located at

$$
\frac{\pi}{2}=1.27 \frac{\Delta m_{41}^{2}\left[\mathrm{eV}^{2}\right] L[\mathrm{~km}]}{E_{v}[\mathrm{GeV}]}
$$


For the example value of a sterile neutrino with a mass of $3 \mathrm{eV}^{2}$, and the MicroBooNE baseline of $0.47 \mathrm{~km}$, the maximum will correspond to BNB intrinsic electron neutrino's with an energy of $1.14 \mathrm{GeV}$, close to the peak of the $v_{\mu}$ event rate given in Figure 3.16.
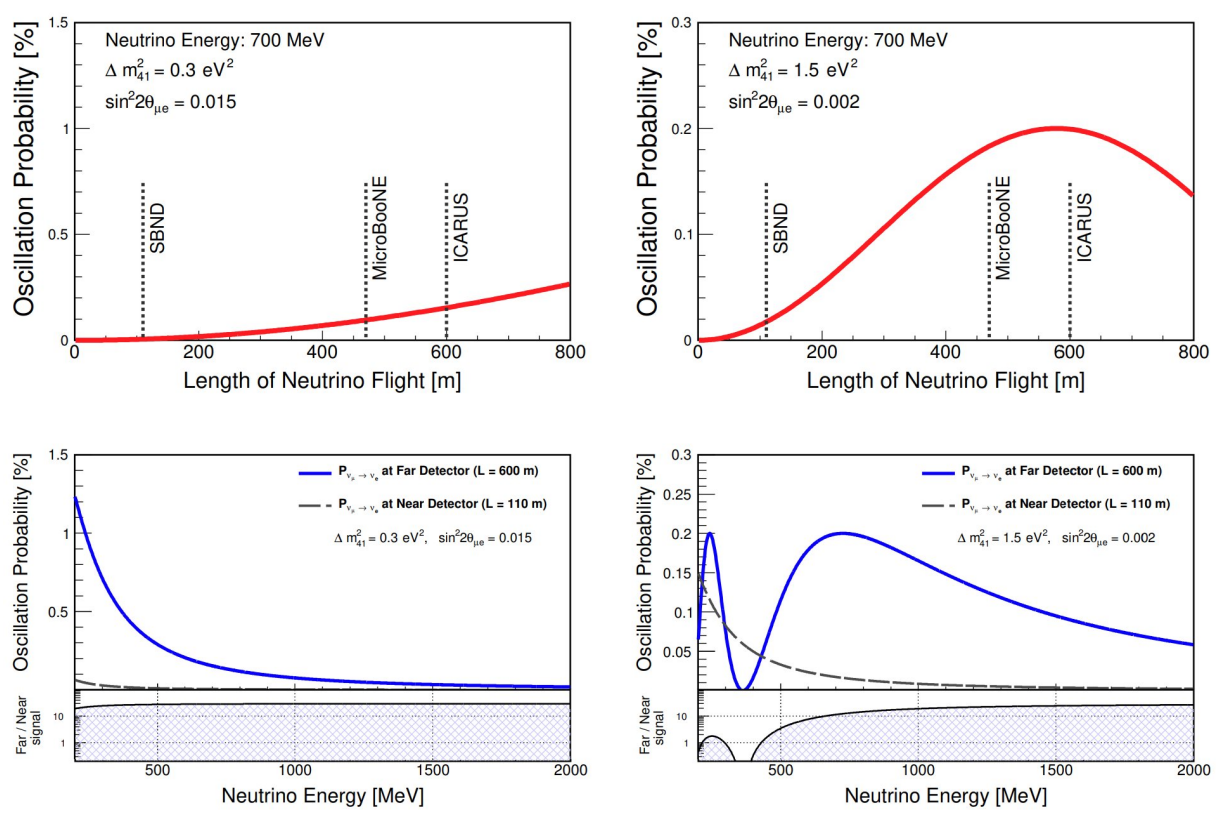

Figure 3.19: Upper panels: $v_{\mu} \rightarrow v_{e}$ oscillation probability for a $700 \mathrm{MeV}$ neutrino as a function of the baseline for two different benchmark points in a $3+1$ sterile neutrino scenario. Lower panels: $v_{\mu} \rightarrow v_{e}$ oscillation probabilities, at $110 \mathrm{~m}$ and $600 \mathrm{~m}$, as a function of the neutrino energy for the same benchmark points. The far-over-near ratio of appearance probabilities is also shown. Figure from [69]. 


\section{4}

\section{Triggering \& Event Reconstruction}

The Time Projection Chamber and light collection systems were introduced in the previous chapter. Especially the 8256 signal channels corresponding to the sensing wires contain a wealth of information. This chapter deals with the processing of the raw detector signals into analysable data products that are used in the future chapters to study cosmic activity and neutrino interactions. The first section focuses on the triggering mechanisms and introduce different data streams. The second section will focus on the TPC and PMT reconstruction that is applied to all data events in order to create high level reconstructed objects 


\subsection{Trigger}

When a neutrino bunch of the accelerator crosses the MicroBooNE detector, it is still unlikely that a neutrino interaction will happen due to their tiny cross section. One BNB bunch contains approximately $1.2 \times 10^{12}$ protons. The flux of neutrinos coming out of the target at the MicroBooNE baseline is given in Table 3.1 and is roughly $5.7 \times 10^{-10} \mathrm{o} / \mathrm{cm}^{2} /$ POT. The frontal surface of the TPC is $\approx 60 \times 10^{3} \mathrm{~cm}^{2}$. Combining those gives a neutrino flux of $\mathcal{O}\left(50 \times 10^{6}{ }^{\circ}\right)$ 's per bunch crossing the TPC. This leads to approximately one neutrino interacting with the argon every 600 bunches. To reduce the number of data events that need to be stored, a certain amount of optical activity - described in the next the next sections - is required. Nonetheless, the stored events are still dominated by background-only events in which the light originates from cosmic activity coincidental with the beam spill. Figure 4.1 illustrates the difference between the two types of events. The cosmic-triggered background events are accounted for in MicroBooNE by taking data while the beam is off, this leads to two trigger types:

1. Data acquired with beam on and triggered by the light system (Beam On), sometimes referred to as "BNB On" to specify the beam trigger.

2. Data acquired with beam off but with the same run configuration and trigger as Beam On (Beam Off). Sometimes referred to as "BNB Off" or "External triggered event" (EXT).

\subsubsection{Hardware triggering}

The first step in MicroBooNE's readout chain is the arrival of a signal telling MicroBooNE to read out an event. This signal can be from the accelerator clocks (for BNB and NuMI) or from a function generator in the trigger rack that produces pulses at a set frequency, which is how Beam Off events are recorded. The trigger board sends a signal to all of the individual readout crates telling them to read out their data, and the trigger board also reads out data which contains the trigger type, and the time the trigger signal was received. 


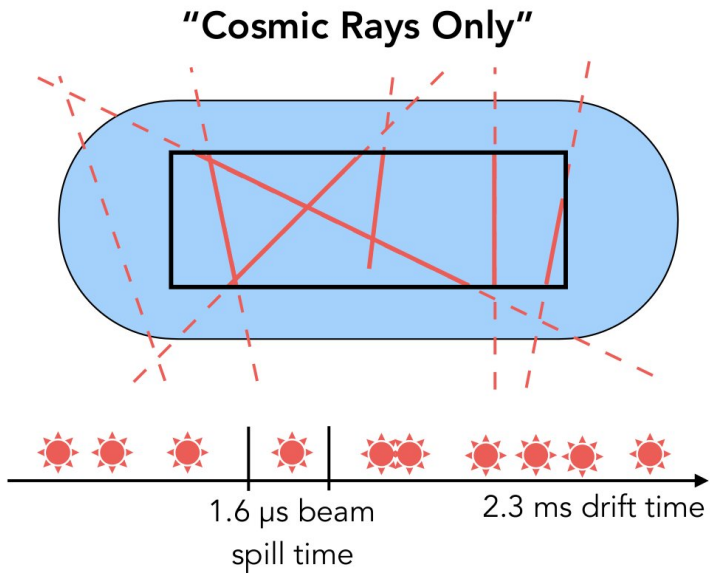

(a)

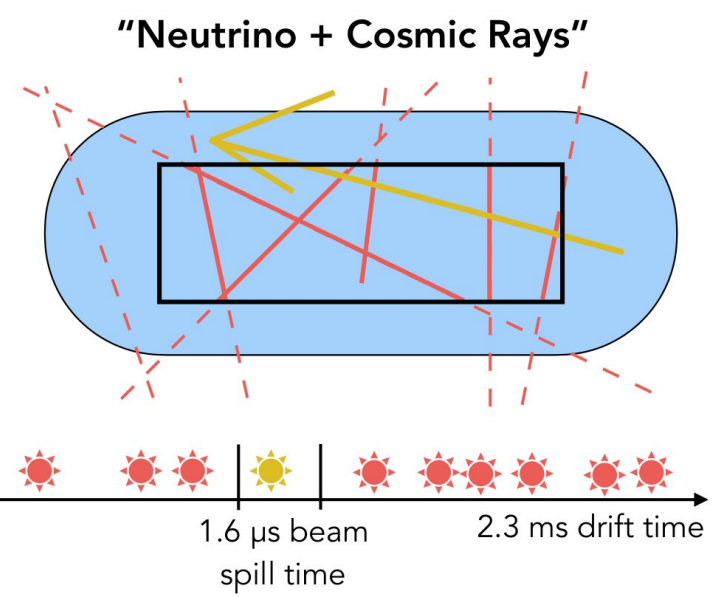

(b)

Figure 4.1: (a) Example of a cosmic activity event that was saved because of the coincidence of a $1.6 \mu$ s accelerator BNB signal and light detected by the PMTs inside the spill window. (b) Example of a neutrino triggered event where the light was coming from a neutrino interaction. Not that in this example the neutrino interaction took place inside the cryostat but outside of the TPC and is therefore still a background. Figure from [58].

The accelerator signals are emitted in pairs. The first signal, the early signal, is used to veto Beam Off triggers shortly before beam triggers, avoiding overlapping triggers causing the loss of beam exposure. The second signal is then used to trigger the actual readout.

Although the MicroBooNE TPC readout is completely unbiased - not zero-suppressed and every time tick is saved - the PMT readout is not. PMT data is only saved for time windows defined by "discriminators" (see below).

\subsubsection{Software triggering}

PMT data is recorded for each event in two different configurations characterised by their length and the level of suppression:

1. Beam discriminators: In the case of BNB triggers, the beam discriminator opens the data collection across all PMTs simultaneously by replicating the trigger signal and sending it to the PMT readout boards. Neutrinos are expected to arrive $\approx 4 \mu$ s after this window is opened and the duration of this beam-gate window is $23.4 \mu$ s (1500 optical ticks, 1 tick $=15.625 \mathrm{~ns})$. 
2. Cosmic discriminators are used outside the $23.4 \mu \mathrm{s}$ window. The cosmic discriminator is designed in order to reduce the amount of recorded data over an extensive interval surrounding the triggered event. Discriminated waveforms are read out for $4.8 \mathrm{~ms}$, corresponding to the TPC readout window: $[-1.6,+3.2] \mathrm{ms}$. The cosmic discriminator only saves waveforms above a threshold of 130 ADC counts, corresponding to approximately 6.5 Photo-Electrons (PE). Every recorded cosmic discriminator saves 40 samples $(\approx 0.6 \mu \mathrm{s})$. A dead-time of 45 samples follows every time a cosmicdiscriminated waveform is recorded.

Accelerator signals are carefully timed such that the neutrinos in the $1.6 \mu$ s long BNB spill passes through MicroBooNE during this $23.4 \mu$ s window. This happens approximately five times a second when the BNB is fully operational.

As the majority of hardware triggered events do not contain a neutrino interaction, a software trigger is applied to determine in real-time if the event has to be recorded or not. The software trigger uses optical waveforms from the PMT system to look at light activity in time-coincidence around the $1.6 \mu$ s beam-spill. This selection takes place after the TPC data has been sent from the readout crates to the DAQ machines. 


\subsection{Event reconstruction}

In this work, both the optical system and TPC charge readout are used. Section 4.2.1 explains how high level data products are created from PMT signals. The reconstruction of the charge readout happens in two stages. First the raw signals are converted to the number of ionisation electrons on a certain wire in function of time, described in Section 4.2.2. Second, this product is used as the input for high level reconstruction to objects such as vertices, tracks and showers (Section 4.2.3).

\subsubsection{Optical reconstruction}

The optical reconstruction takes raw PMT waveforms as input. From these, flashes are reconstructed. Flashes represent coincidental optical activity across several PMTs, usually caused by a single interaction in the TPC. Light is collected on 328 inch PMTs, using a low $(\approx 20 \mathrm{ADC} / \mathrm{PE})$ and a high $(\approx 2 \mathrm{ADC} / \mathrm{PE})$ gain readout [70]. The first step performed in the optical reconstruction is the merging of these two streams into a "saturation-corrected" waveform which tries to correct saturated high-gain pulses by using information from the low-gain channel.

Baseline Estimation and pulse finding The baseline estimation of the waveform depends on the discriminator that triggered it. A constant baseline - or pedestal - is assumed for the cosmic discriminator while the longer window of the beam discriminator allows a more accurate time dependent baseline estimate. This takes care of estimating the right pedestal if the waveform undershoots or if there are possible fluctuations in the signal baseline. Once we have the pedestal, an algorithm is run that looks at the waveform ADC counts. Pulses above a configurable threshold are selected and passed to the next module for "hit" reconstruction.

Optical hit reconstruction The optical hit reconstruction creates data products from the pulses. An optical hit is meant to represent optical activity in a single PMT, containing the rising edge time and the photo-electron count. 
Flash reconstruction Flash reconstruction clusters optical hits and sums the corresponding Photo-Electrons across all PMTs. The time range is divided into $30 \mathrm{~ns}$ intervals and coincidental optical hits across at least three PMTs are looked for. Once coincident hits are found, an integration window of $8 \mu \mathrm{s}$ is applied in order to collect all the late light associated with the interaction. To avoid that another flash is claimed by late light, a $8 \mu$ s dead time window is applied during the flash integration window. If multiple candidate flashes within this time window can be constructed, the algorithm saves the one with the highest number of reconstructed PE. An event display of such a flash is given in Figure 4.2.

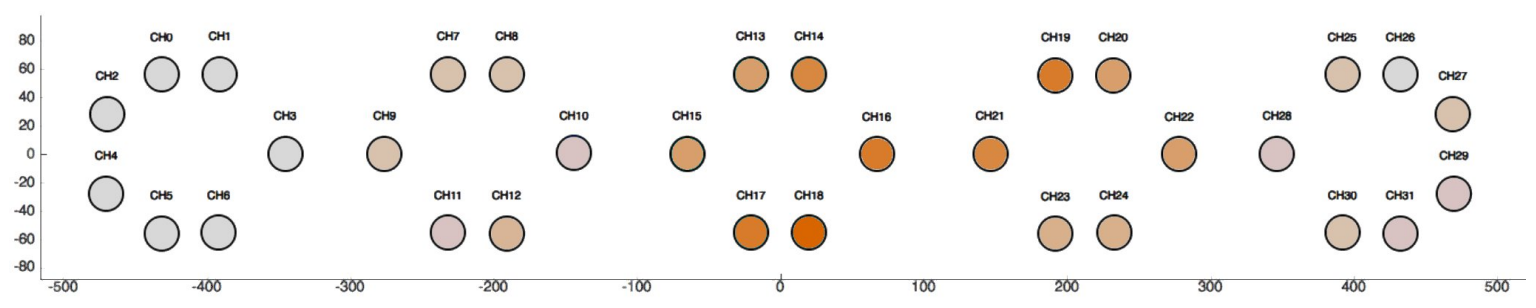

Figure 4.2: Example of a flash consisting of multiple PMT hits combined. The orange represents the intensity of the light arriving in each PMT, integrated of $8 \mu$ s.

We are usually interested in the flashes that happen during the beam spill window. Given the dead time window, it is impossible to have multiple reconstructed flashes during one 1.6 $\mu \mathrm{s}$ BNB spill. The flash reconstruction also performs a constant 2 PEs per PMT background subtraction. This correction compensates for the $250 \mathrm{kHz}$ dark noise and is integrated over an $8 \mu$ s window: $8 \mu \mathrm{s} \times 0.25 \mathrm{MHz}=2$ PEs. This is applied to all reconstructed flashes.

\subsubsection{TPC signal processing}

The processing of the raw TPC waveforms of the sensing wire signals, illustrated in Figure 3.8 , is a crucial first step to accurately reconstruct particle interactions in the detector. Figure 4.3 serves as a demonstration for the need of signal processing before reconstruction is feasible. The image shows a neutrino interaction as seen on the first induction plane during different steps of the signal processing [71].

Three elements come into play for signal formation on TPC wires [71]: 


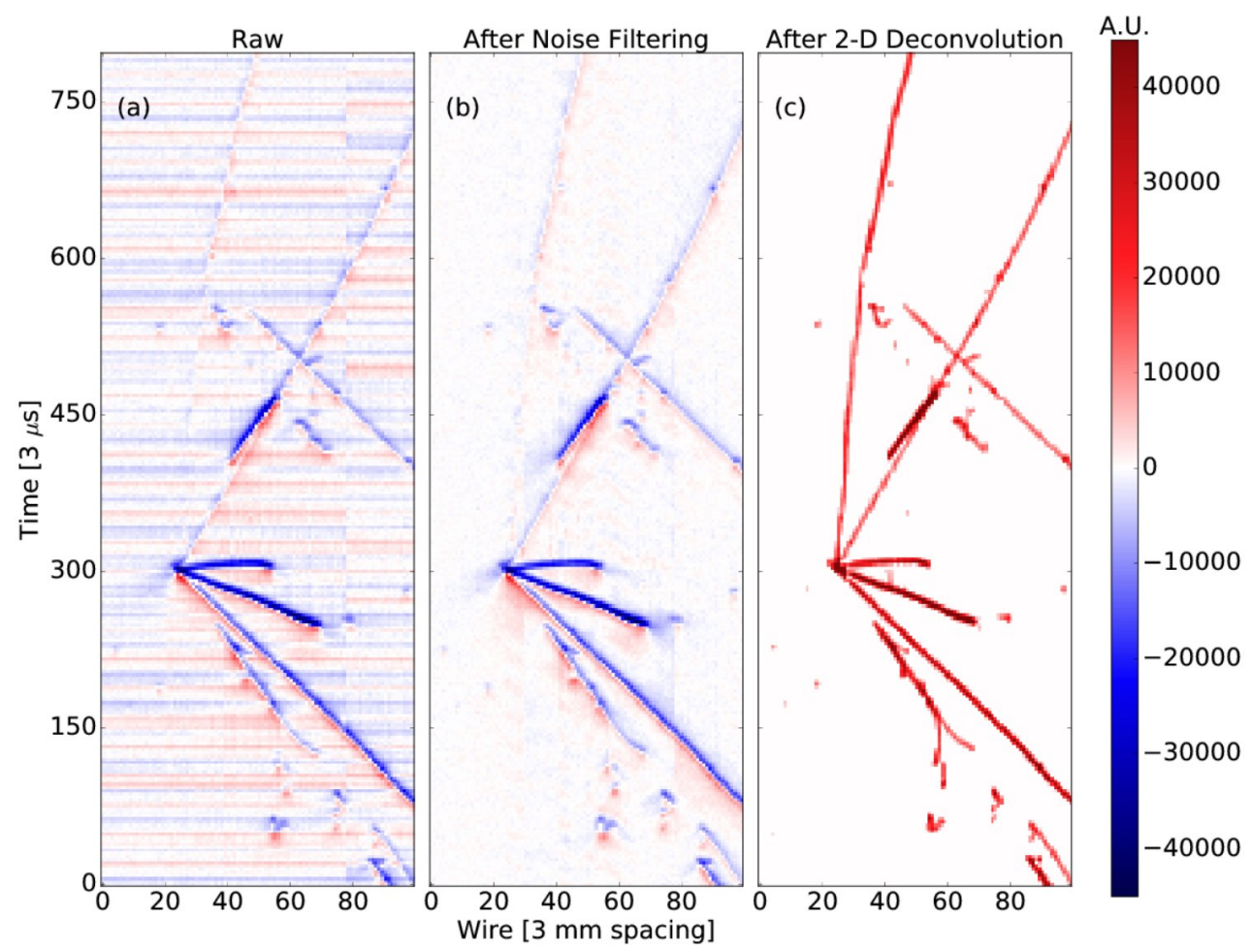

Figure 4.3: An example neutrino candidate event display from MicroBooNE data (event 41075, run 3493) showing a $U$ plane view. (a) The raw waveform image in units of average baseline subtracted ADC scaled by 250 per $3 \mu \mathrm{s}$. (b) The image after software noise-filtering in units of average baseline subtracted ADC scaled by 250 per $3 \mu \mathrm{s}$. (c) The image after $2 D$ deconvolution in units of electrons per $3 \mu \mathrm{s}$. Prolonged signals associated with near-vertical tracks, such as the one at the top left of each event display window, are recovered after the deconvolution step. Additionally, the image quality near the neutrino interaction vertex improves after the $2 D$ deconvolution, which is expected to lead to improvements in the pattern recognition. Figure from [71].

1. The distribution of the ionisation charge cloud produced by charged particles travelling through the TPC. During their drift, electrons repel each other, leading to a diffuse signal at the anode plane, depending to the location of the interaction.

2. The field response describing charge collection and induction on the TPC wires as ionisation electrons drift through the wire planes. Drifting electrons induce current over an expanse of nearby wires, introducing the possibility that the waveform produced by electrons passing by a particular wire affects the one produced by electrons passing a nearby wire. 
3. The response of the electronics. The amplification and shaping of the induced current on the TPC wire in the front-end electronics. Current induced on the TPC wires is amplified and shaped by low-power, low-noise ASICs immersed in the liquid argon.

The MicroBooNE noise filtering and signal processing aims to convert the raw digitised TPC waveform into the number of ionisation electrons passing through a wire plane at a given time [72]. In general, the algorithm contains three major steps:

- Noise filtering: Apply noise filters to remove both the inherent electronics noise and external noise. The two largest sources of external noise have been identified as originating from the TPC drift high-voltage power supply and the low-voltage regulators for the front-end ASICs [57].

- 2D deconvolution: Apply a deconvolution to the digitised TPC wire signals. This deconvolution is performed in two dimensions. The first dimension is an analytical analysis of the waveform over time, and the second dimension takes into account the long-range effect of the induction signals across multiple wires.

- Region of Interest (ROI) finding and application: Perform ROI finding with the deconvolved charge distribution. Loose and tight high-pass filters are combined to optimise the purity and efficiency of ROI finding. The identified ROI window is then applied to the deconvolved charge distribution. The ionisation charge is extracted with a linear baseline subtraction for the induction planes, based on the start/end bins of the ROI window.

The final processed signals, each consisting of the number of electrons arriving on a certain wire at a certain time, form the input of the MicroBooNE event reconstruction. For calorimetric purposes, the obtained charge response signals are calibrated before the conversion to deposited energy is made (Appendix A).

\subsubsection{Pandora reconstruction framework}

In order to fully exploit the high granularity offered by TPCs, the Pandora reconstruction framework was developed [73]. Pandora facilitates the implementation of pattern- 


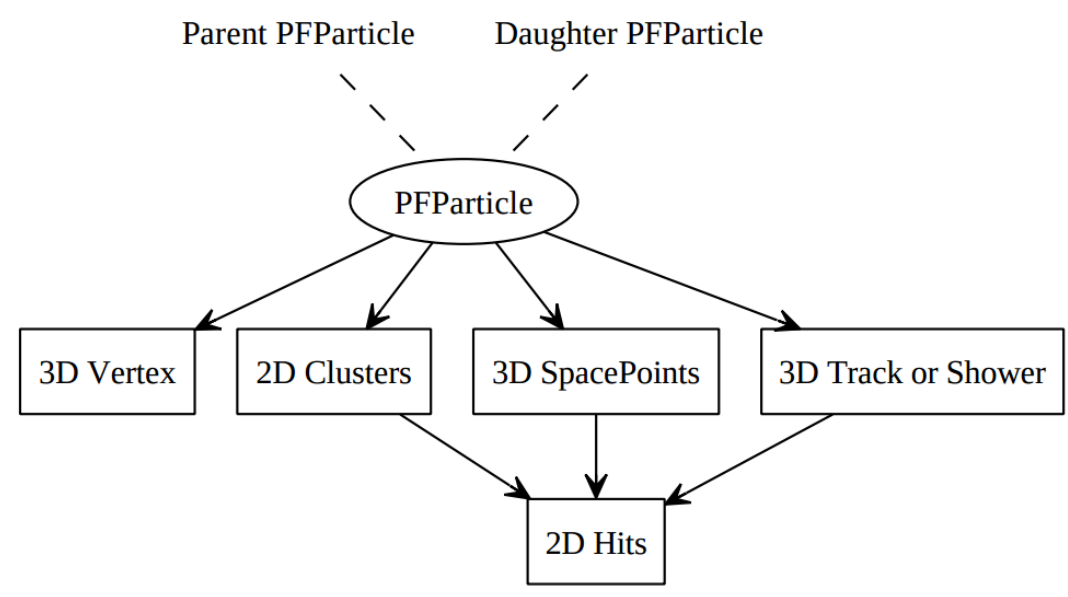

Figure 4.4: The Pandora output data products. The reconstructed particle hierarchy is represented by a dashed line, associated objects are represented by solid arrows. Figure from [73].

recognition algorithms while promoting a multi-algorithm approach in which individual algorithms each address a specific task in a particular topology. The wire waveforms in the $4.8 \mathrm{~ms}$ readout window are taken as input of the reconstruction. First, a Gaussian distribution is fitted to each peak in the signal processed waveforms, called a $2 D$ hit.

Two subsequent reconstruction paths have been created for use in the MicroBooNE analysis:

- PandoraCosmic is a track-oriented selection and aims at unambiguously tagging cosmicray muons. Afterwards, a cosmic-removed hit collection is created.

- PandoraNu aims to identify a neutrino interaction vertex and uses it to aid the reconstruction of all particles, tracks and showers, emerging from the vertex position. A parent neutrino particle is made and the reconstructed particles are added as daughters, as seen in Figure 4.4.

Pandora slicing, clustering \& vertexing The creation of a "slice" is the first step of the Pandora processing. A slice is a collection of reconstructed particles which belong to the same interaction. Examples of this are a cosmic muon and its Michel electron, or a muon and a proton in a $1 \mu 1 p$ neutrino interaction. Note that although a slice aims to represent an instantaneous $(<\mathcal{O}(\mu \mathrm{s}))$ physics interaction in the TPC, the collection time of the corresponding 

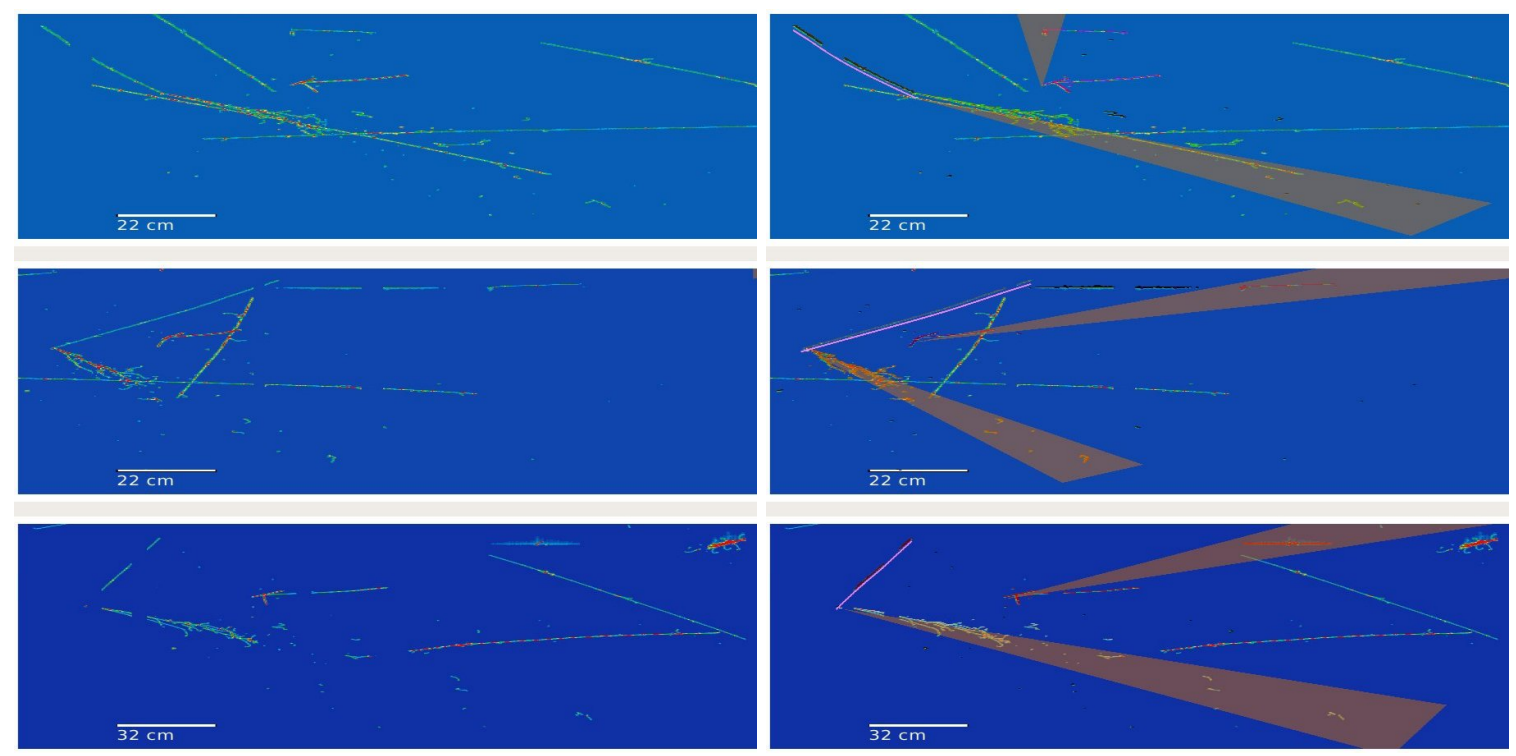

Figure 4.5: Simulated electron neutrino event with $E_{v}=2.4 \mathrm{GeV}$ in MicroBooNE. The three planes are shown, the upper two panels correspond to the induction planes $U, V$; the bottom panel is the collection plane $Y$. In the right panels, the Pandora reconstructed objects are superimposed on the image. Pandora correctly identified the electron shower (orange) and the charged pion track (purple).

charge can be $\mathcal{O}(\mathrm{ms})$ due to the drift time.

To produce slices, the PandoraCosmic pattern recognition is first run over all hits, aiming to construct muon tracks and associated $\delta$-rays and Michel electrons under the cosmic hypothesis. At this stage, obvious cosmic activity (through-going or out-of-time muons) is tagged using geometric information. The remaining hit collection is used as input to the PandoraNu pattern recognition which reconstructs slices under the neutrino hypothesis. Each slice is now reconstructed under both the cosmic hypothesis and the neutrino hypothesis.

In order to reconstruct the interactions in $3 D$, Pandora needs to match the information from at least two different views - wire planes - and create a neutrino vertex. Pandora clusters the $2 D$ hits in each slice and in each plane separately. Then, a number of $3 D$ candidate vertices is created by finding positions that project down onto the ends of the available $2 D$ clusters. All possible vertex candidates are fed into a Support Vector Machine (SVM) vertex selection, and the candidate with the highest classifier response is chosen. This $3 D$ vertex is 
used to split any existing clusters that straddle the vertex. Then, the cluster matching algorithms are run, where the clusters are compared between views and modified to improve the matching [73].

Pandora particle hierarchy The outcome of the Pandora reconstruction is a list of reconstructed Particle Flow Particles (PFParticles), illustrated in Figure 4.4. A PFParticle is constructed by combining up to three $2 D$ Cluster objects, one for each of the three wire planes. Each PFParticle has a vertex position and is associated with a set of reconstructed $3 D$ points that carry the charge information of the corresponding $2 D$ hits, called SpacePoints.

The PFParticles in the slice are placed in a hierarchy, which identifies parent-daughter relationships and describes the particle flow in the observed interactions. A neutrino PFParticle is placed at the root of the hierarchy by the PandoraNu reconstruction path. The neutrino will have at least one, and often multiple daughters. For example a track originating from a muon. If this muon decays to a well-reconstructed Michel electron shower, the shower will be a daughter of the muon track.

As will be discussed in Chapter 6, a typical event contains approximately 4 of those Pandora neutrino slices per event. It should be understood that only a tiny fraction of these neutrino candidates are corresponding to neutrino induced activity and the majority originates from cosmic activity, as will be further discussed in Chapter 6.

Shower \& track fitting Each PFParticle is attributed a continuous score to categorise it as either track-like or shower-like. The nature of the object is determined via a Support Vector Machine, which uses topological information from the hits. Shower-like particle will be scored close to 0 while track-like particles will have high responses close to 1 . Depending on the score, for every particle, a shower- or a track-like data product is created.

If interpreted as a track, Pandora processes the PFParticle with a sliding linear fit procedure - described in [73] - that returns the $3 D$ position and direction at each point along the trajectory. For each point the charge deposited along the trajectory $(\mathrm{d} Q / \mathrm{d} x)$ and dis- 
tance from the track-start are recorded. This procedure allows to accurately measure $\mathrm{d} x$, including small deflections due to the particle's trajectory and space charge effect offsets. $\mathrm{d} Q$ is measured, incorporating a position- and field-dependent charge calibration (see Appendix A). For tracks, the conversion from $d Q / d x$ to $d E / d x$ is performed by applying the inverse Modified Box model [74]. This model takes into account the non-linear dependence of the local ionisation density on the electron recombination.

The shower-like interpretation of the reconstructed particle builds a $3 D$ cone around the hit collection with a defined length, opening angle and $3 D$ direction. Furthermore, the shower energy is reconstructed and calibrated, the same set of algorithms is used as was developed and validated using $\pi^{0}$ reconstruction [75]. Showers are also fitted using a Kalman filter procedure [76]. The main trunk of the shower is built by excluding hits that are longitudinally or transversely displaced from it; the output of this fit is a track object. Thus, the calorimetric tools described above to characterise the $\mathrm{d} E / \mathrm{d} x$ become available for showers as well.

Figure 4.5 illustrates the result of the reconstruction on the different planes for a simulated high-energy electron-neutrino event. The electron neutrino interaction products, an electron shower and a charged pion track are both reconstructed and overlaid on the charge deposits in the right panel.

\subsubsection{Event simulation}

Two categories of simulated events will be used in this work:

1. Cosmic simulated events In Chapter 5, cosmic activity in the MicroBooNE detector will be studied. The simulation of the cosmic activity from the galactic flux of primary particles to the charge deposits in the detector relies on a computationally expensive combination of CORSIKA and GEANT [64, 65].

2. Overlay events This type of events contains a GENIE simulated neutrino interaction in the cryostat or surrounding material. To save computation time and improve the quality of the simulated samples, the simulated neutrino charge deposits are overlaid 
with cosmic data collected with the unbiased trigger. This trigger type stores an event outside of the beam triggered time windows and does not require any optical activity in a specific region.

Reco-truth matching For simulated events, the reconstructed information is traced back to the underlying truth information. This goal is achieved by mapping the reconstructed charge deposits on the different planes - the hits - to the GEANT parent particles responsible for it. From the hit level it is therefore possible to connect reconstructed PFParticles with a known purity and completeness back to the simulated neutrino interaction product. Furthermore, it is possible the tag the amount of charge originating from the overlaid cosmic data. A PFParticle - either track- or shower-like - is said to be back-tracked/matched to a simulated particle if has more hits in common with it than with any other simulated particle - or cosmic activity - in the event. This does not guarantee that the simulated neutrino daughter particle is properly reconstructed. It is still possible that a part is missing, that is merged with a less energetic simulated particle or that it contains a contamination from cosmogenic backgrounds. 


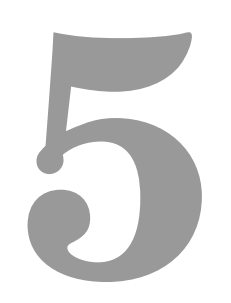

\section{Cosmic-Ray Measurements}

This chapter describes a cosmic-ray rate study using the three detector sub-systems of MicroBooNE independently. Section 5.1 describes the Monte Carlo (MC) cosmic-ray simulation that will be compared with data throughout the chapter. In Section 5.2, the Time Projection Chamber (TPC) is used to measure the muon $\left(\mu^{ \pm}\right)$flux, integrated over muon energies and direction. Furthermore, the angular distributions are compared with simulation and the muon reconstruction resolution is studied using MC. The Photo-Multiplier Tube (PMT) system is used in Section 5.3 to independently cross check the TPC-based muon rate measurement. Since February 2017, MicroBooNE has been equipped with CRT panels at the top, bottom, anode and cathode sides. In Section 5.4, recent data, summer 2018, is used to study the muon rate using the CRT bottom panel, which serves as a first physics demonstration of the CRT system. A detailed description of each of the three systems can be 
found in Chapter 3. Section 5.5 compares the results of the independent rate measurements with existing literature.

\subsection{The cosmogenic flux simulation}

Cosmic rays are produced when galactic protons - or heavier elements like He up to Fe - interact with the Earth's atmosphere. This interaction leads to extensive air showers, containing a large amount of cosmic particles, referred to as primaries. The composition and the flux of these cosmic particles depends on the location - latitude, longitude and altitude - as well as the amount of shielding between the atmosphere and the detector.

As described in Section 3.2.1, the TPC technology requires a readout window of $\mathcal{O}(\mathrm{ms})$. In combination with the large detector size and the lack of a significant overburden, this results in a large amount of cosmic-induced charge deposits per event. Additionally, the low neutrino event rate means that the majority of recorded events will consist of cosmic activity, resulting in an important background for neutrino interaction measurements. Detailed simulation studies are crucial in order to quantify the cosmogenic activity [77].

\subsubsection{Geometry}

Fermilab is geomagnetically located at a latitude of $41^{\circ} 50^{\prime} 15^{\prime \prime}$ North and a longitude of $88^{\circ} 16^{\prime} 10^{\prime \prime}$ West. The MicroBooNE TPC is located inside an open concrete pit, $6 \mathrm{~m}$ underground, is at an elevation of $228 \mathrm{~m}$ above mean sea level [77]. The MicroBooNE geometry simulation includes the Liquid Argon Test Facility (LArTF) building, the roof, the concrete pit and the cryostat. The platform and electronics racks are also included as shown in Figure 5.1, left. The MicroBooNE dimensions and coordinate system are defined in Figure 3.6. The cryostat, the surroundings and their effect on incident cosmic particles are given in Figure 5.1. The right panels highlight the important features, using the end points of simulated cosmic particles. The colour scale corresponds to the density of stopping particles in certain elements. 

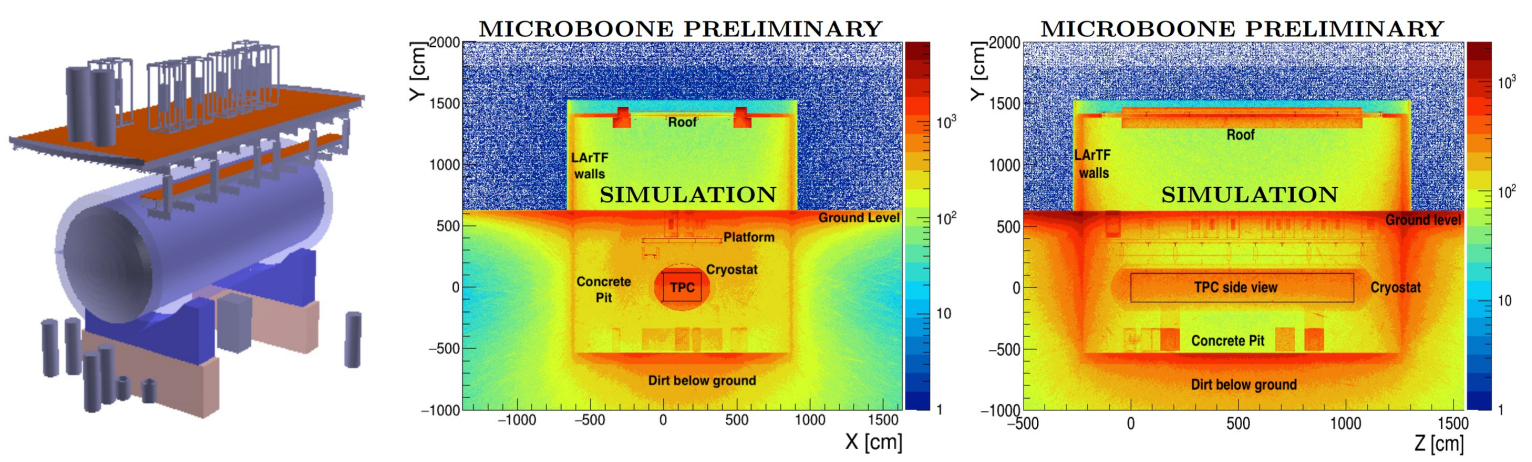

Figure 5.1: (Left) Simulation of the cryostat environment in the concrete pit of the LArTF building, including the platform above the detector hosting several electronics racks. (Right) End point distribution of all cosmic particles for both the $Y X$ and $Y Z$ projections. The particles are simulated starting at the plane $y=18 \mathrm{~m}$ and going downwards. End points above $y=18 \mathrm{~m}$ are due to re-scattering. The different geometry elements stopping most particles are clearly visible. Figure from [77].

\subsubsection{Cosmic-ray generators}

Two different MC generators for cosmic rays have been used in MicroBooNE [77]. Both generators assume a configurable flux of incoming extra-terrestrial particles and propagate this to a collection of particles, referred to as primaries in this chapter, placed on a surface $18 \mathrm{~m}$ above the TPC.

Cosmic-RaY shower library (CRY) is set-up to take the latitude dependence into account. No seasonal variations or longitudinal location effects on the flux are included. All particles are simulated under the assumption of $0 \mathrm{~m}$ elevation (sea level). This underestimates the muon flux by $\approx 5 \%$, while underestimating the primary neutron, proton and electron flux by 20 to $30 \%$ [12]. The CRY particle sampling is based on pre-computed tables, assuming only protons as incident particles. Another shortcoming in CRY is that it uses large bin widths to store energy spectra, which results in inaccurate sampling and step-like structures [78].

COsmic Ray SImulations for KAscade (CORSIKA) is MicroBooNE's default generator for detailed simulation of extensive air showers initiated by high-energy cosmic ray-particles. It allows to take into account the location - latitude, longitude and elevation - of Fermilab. CORSIKA also includes a composite incoming flux, consisting of multiple particle 
types instead of only protons. Furthermore it enables testing alternate models for particle shower development such as GHEISHA and FLUKA. MicroBooNE uses the CORSIKA version 7.4003 for its cosmic background simulation [64]).

CORSIKA was chosen as the default generator, due to the extra flexibility offered over CRY, such as a modifiable incoming flux of extra-terrestrial particles. Two different incoming galactic flux models are used:

1. Proton-only. In this model, only cosmic protons are assumed to contribute to the Earth's cosmic-ray flux. The flux used is [12]:

$$
\Phi(E)=1.8 \times 10^{4}(E[\mathrm{GeV}])^{-2.7} \frac{\text { nucleons }}{\mathrm{m}^{2} \mathrm{~s} \mathrm{sr} \mathrm{GeV}}
$$

2. Constant Mass Composition (CMC). According to this model, the primary cosmic incident flux contains substantial amounts of both light and heavy elements. The CMC model parametrises the spectra of each component, which are combined to reproduce the full primary particle spectrum [79]. In this model, the flux is given by:

$$
\Phi_{A}(E)=K_{A} E^{-\gamma_{A}},
$$

where $E$, the energy of the cosmic ray, is expressed in units of $\mathrm{GeV}$ and the index $A$ corresponds to the type of incident particle.: protons, $\mathrm{He}, \mathrm{N}, \mathrm{Mg}$ and Fe. The spectral index $\gamma_{A}$ is assumed type independent and is set to 2.71. $K_{A}$ is a particle type dependant normalisation factor and takes the values $1.72 \times 10^{4}, 9.2 \times 10^{3}, 6.2 \times 10^{3}$, $9.2 \times 10^{3}$ and $6.2 \times 10^{3}$ for the five components. The unit of $K_{A}$ is such that the flux $\Phi(E)$ has the units $\frac{\text { nucleons }}{\mathrm{m}^{2} \mathrm{ssrGeV}}$.

Generator comparisons Figure 5.2 shows the energy distributions for the two generators described above. If only galactic protons are considered as primary particles, CORSIKA predicts an increase in the integrated muon flux of $\approx 6 \%$ over CRY, both for GHEISHA and FLUKA propagation models. Employing the FLUKA interaction model moves the muon energy spectrum towards lower energies. When the Constant Mass Composition (CMC) 
model is included in CORSIKA+FLUKA, an additional increase of $26 \%$ in the integrated muon flux is predicted, without altering the energy distribution significantly. The integrated values are summarised in Table 5.1
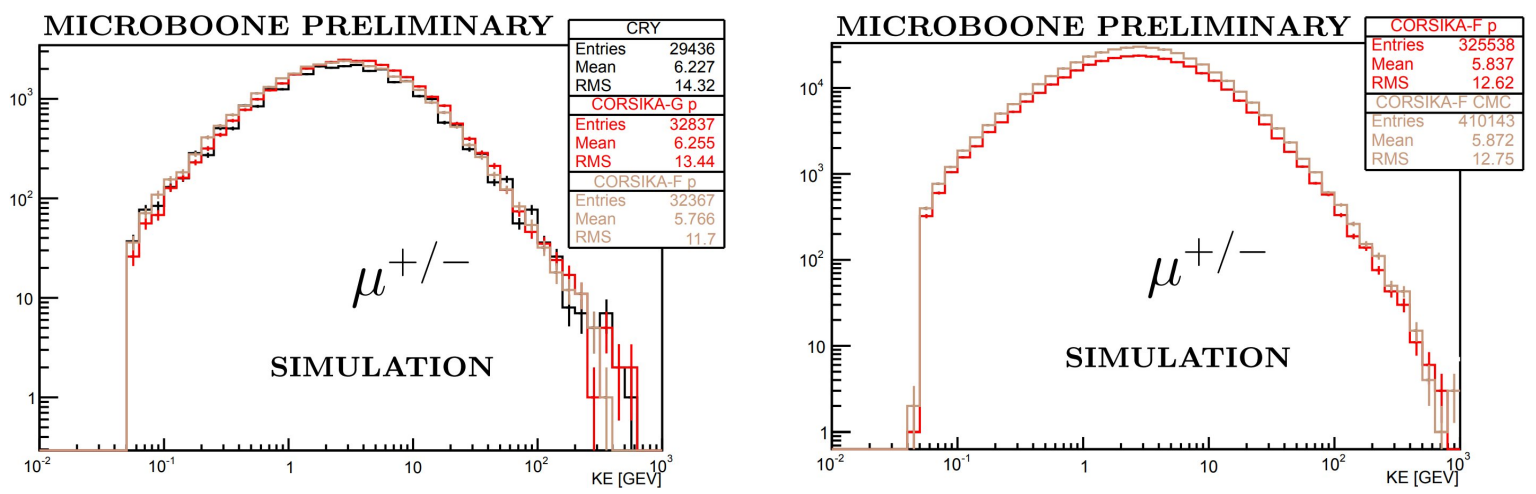

Figure 5.2: Comparisons of the energy distributions of atmosprheric muons for different cosmic generators through the top surface of the active TPC. (Left) Using CRY, CORSIKA+GHEISHA and CORSIKA+FLUKA, containing 2000 simulated $4.8 \mathrm{~ms}$ events. (Right) Comparing CORSIKA with and without the CMC contribution, containing 20000 simulated $4.8 \mathrm{~ms}$ events. [77].

Table 5.1: Integrated muon fluxes for different cosmic simulations. For CORSIKA, the correct elevation is included. For CRY, the rates are corrected to the elevation of Fermilab after the simulation. [77]

\begin{tabular}{cccc}
\hline & CRY & CORSIKA(FLUKA) & CORSIKA(FLUKA)+CMC \\
\hline$\mu^{ \pm}$flux $\left[\mathrm{m}^{-2} \mathrm{~s}^{-1}\right]$ & $120.0 \pm 0.2$ & $127.7 \pm 0.2$ & $160.9 \pm 0.3$ \\
\hline
\end{tabular}

\subsubsection{Simulated muon rate}

As mentioned before, due to several caveats of the CRY generator, studies in the rest of the chapter will be performed using CORSIKA. The default configuration is the proton-only incident flux model in combination with the FLUKA propagation model.

Muons and anti-muons generated by CORSIKA are propagated down from a plane at $y=18 \mathrm{~m}$ through the environment and the detector geometry using GEANT [80]. The muon trajectory is described by a set of interaction points with corresponding energy losses. The trajectory ends when the muon decays in flight, undergoes decay in orbit or nuclear 


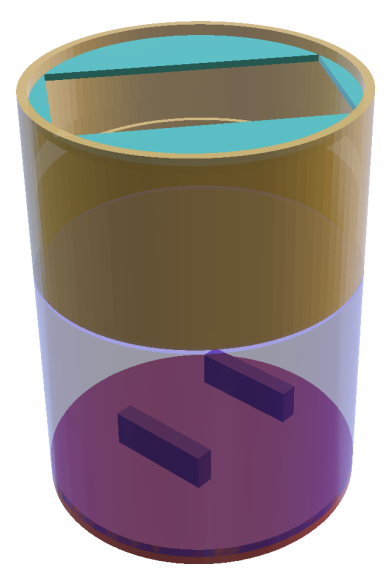

Figure 5.3: Concrete elements in the LArTF simulated geometry: cylindrical wall around the building (brown), floor of the pit and set of two concrete stands for the cryostat (purple), roof with opening through which MicroBooNE was initially installed (cyan). The density of the concrete was varied by $\pm 30 \%$ to estimate potential inaccuracies in the modelling.

capture. The rate of muons, predicted with CORSIKA, which intersect the active TPC volume is $(4388 \pm 1) \mathrm{Hz}$. The cosmic muon rate $^{1}$ crossing the top panel of the TPC is $(111.5 \pm 0.2) \mathrm{Hz} / \mathrm{m}^{2}$.

Building geometry variations The rate inside the TPC is reduced by $13 \%$ compared with the rate $18 \mathrm{~m}$ above the TPC. This reduction is caused by the argon in the cryostat outside of the TPC, which is well modelled, and the shielding provided by the Liquid Argon Test Facility (LArTF) and the surrounding ground. The majority of the LArTF shielding originates from the concrete elements, depicted in Figure 5.3. As it is difficult to reproduce the building geometrical properties (wall thickness, concrete density ...), an inaccurate geometry can lead to an uncertainty on the muon flux inside the TPC. In order to estimate potential mismodelling of the building geometry, large variations in the concrete density were used. This should englobe any additional material or variations in the building structure, in a way that was practical for the simulations. Two samples were generated, one with a $30 \%$ increase in wall and roof density and one with a $30 \%$ reduced wall and roof density. The $30 \%$ was chosen as a conservative estimate estimation of how general mis-modelling of the cryostat surroundings impacts the measurements.

\footnotetext{
${ }^{1}$ The errors quoted are statistical only, systematic errors will be discussed at the end of Sections 5.2 and 5.3.
} 
For the simulation with the increased concrete density, the muon flux through the TPC is $(4309 \pm 2) \mathrm{Hz}$. In the case of reduced amount of shielding the flux is $(4468 \pm 2) \mathrm{Hz}$. This increase and decrease of $30 \%$ leads therefore to an effect of approximately $2 \%$. This variation is an overestimation of what realistically can be expected, and will serve as a conservative estimate of the systematic uncertainty related to the building geometry.

Simulated muon energy The left panel of Figure 5.4 shows the energy of muons that enter the TPC. Reconstruction threshold will restrict the measurement in Sections 5.2 and 5.3 to muon with an initial kinetic energy of approximately $200 \mathrm{MeV}$ or higher. Energy threshold effects are further discussed in Appendix C.3.

In Chapter 6, a set of cosmic rejection tools will be developed. Stopping muons are an especially hard category of cosmic backgrounds for neutrino interactions. In anticipation, the right panel of Figure 5.4 shows the fraction of stopping muons in the TPC. There are two processes that contribute to this fraction:

- Decay: For $\mu^{+}$, this is the only relevant process. For $\mu^{-}$, this happens in approximately $27 \%$ of the cases. The decay leads to a low energetic positron/electron shower, referred to as Michel electrons.

- Absorption: This is the dominant contribution for negatively charged particles, and does not lead to a clear signature $\left(\mu^{-}+p \rightarrow n+v_{\mu}\right)$.

Muons entering the TPC, generated with a kinetic energy below $1 \mathrm{GeV}$, are likely to stop in it. This is unsurprising since the energy loss $(\mathrm{d} E / \mathrm{d} x)$ of a Minimal Ionising Particle (MIP) in liquid argon is $2.1 \mathrm{MeV} \mathrm{cm}^{-1}$ on average [81]. The diameter of the cryostat housing the $\mathrm{TPC}$ is $381 \mathrm{~cm}$, therefore, a muon entering the cryostat with less than $0.8 \mathrm{GeV}$ will almost certainly stop in it. In addition, muons lose energy before entering the cryostat due to the roof, walls, instrumentation and surrounding ground. 

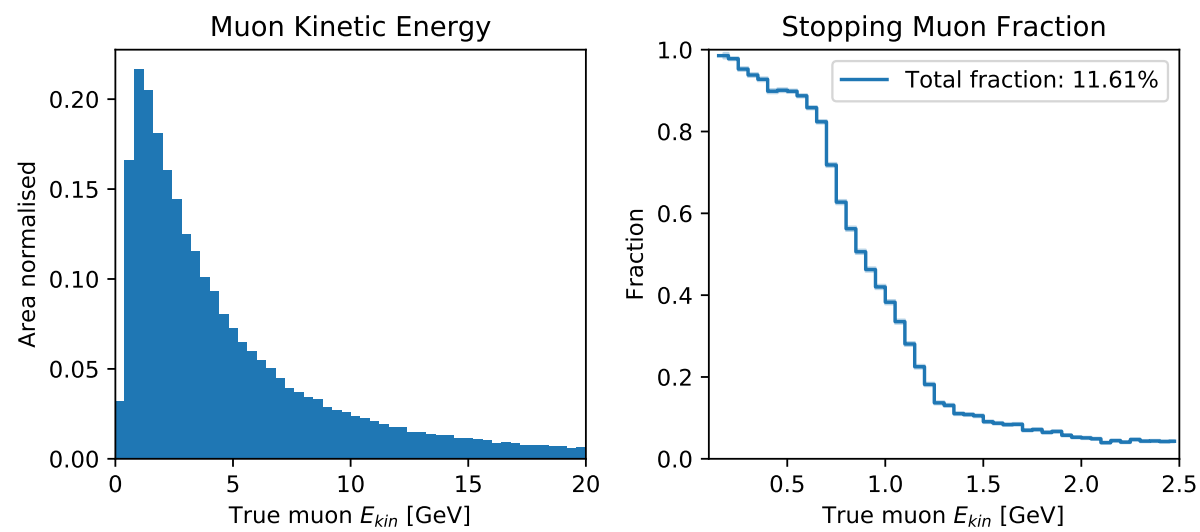

Figure 5.4: (Left) Distribution of kinetic energy, $E_{k i n}$, of simulated muons entering the TPC. (Right) Fraction of simulated muons that stop inside the TPC. The fraction of muons that enter the TPC and stop inside it is $11.6 \%$. Note the different scale on the horizontal axis. 


\subsection{TPC-based muon rate \& reconstruction resolution}

To perform a study of cosmic rays in MicroBooNE, the PandoraCosmic particle reconstruction is used (see Section 4.2.3). This reconstruction is track-oriented, focusing on cosmic-ray muons. In this configuration, reconstructed electromagnetic showers are assumed to be delta rays or Michel electrons and are added as daughter particles to the most appropriate muon track. The reconstructed vertex/start-point for the cosmic-ray muon is chosen, such that the muon is downwards going.

\subsubsection{Reconstruction purity and efficiency}

Purity reconstructed tracks are reco-truth matched to the corresponding simulated cosmicray particle. The purity is defined as the fraction of selected tracks that is matched to a muon. If we consider tracks with a minimal length of $5 \mathrm{~cm}$, the muon purity is $(95.35 \pm 0.03) \%$. The remaining $5 \%$ is explained by mis-reconstruction of neutrons $(1.64 \%)$ and photons $(1.54 \%)$. The remaining $\approx 1.5 \%$ is a combination of protons, electrons and positrons.

- Neutrons. Most tracks of at least $5 \mathrm{~cm}$ arise from neutron inelastic scattering. The initial energy of the neutrons causing these tracks is above $1 \mathrm{GeV}$ and ranges up to multiple GeV.

- Photons. The photons that cause tracks of at least $5 \mathrm{~cm}$ can be primary particles ( $55 \%$ ) or come from bremsstrahlung ( $45 \%$ ). The energy of these photons is mostly below $250 \mathrm{MeV}$ and will, through pair-production, lead to a positron and an electron. These $e^{ \pm}$can lead to reconstructed tracks with a length above $5 \mathrm{~cm}$.

It is possible that two or more reconstructed tracks belong to the same generated muon. This usually happens when a muon crosses an unresponsive detector region or scatters inside the TPC. $9.93 \%$ of reconstructed tracks do not uniquely correspond to a generated muon. Therefore the purity of tracks that uniquely correspond to a muon is $(85.42 \pm 0.03) \%$.

A sample with higher purity is obtained when tracks with a reconstructed length of $>25 \mathrm{~cm}$ are selected. Within this sample, the purity of tracks that uniquely correspond to a muon 
is $(97.71 \pm 0.02) \%$. The fraction of broken muon tracks and tracks reco-truth matched to non-muon cosmic particles are both $\approx 1 \%$. From now on, this purer selection will be used.

Efficiency Of all simulated muon tracks entering the TPC, 93.2\% have a true length of at least $25 \mathrm{~cm}$. The muon reconstruction efficiency for those is $(97.84 \pm 0.05) \%$. Here, we defined:

- Denominator: CORSIKA generated muons with a length of at least $25 \mathrm{~cm}$ inside the TPC.

- Numerator: All these CORSIKA muons that have a reconstructed track with a length of at least $25 \mathrm{~cm}$.

The quoted efficiency is in agreement with a previous data-driven efficiency measurement using an external muon counter system that found $(97.1 \pm 0.1$ (stat) \pm 1.4 (sys) $) \%$ [82].

The inefficiency of $\approx 2 \%$ is explained by simulated tracks inside the TPC that are above $25 \mathrm{~cm}$, but are reconstructed as shorter tracks due to reconstruction imperfections. These include a variety of reconstruction effects, such as: broken tracks, space charge effects and unresponsive detector zones.

\subsubsection{Data and Monte Carlo comparisons for the muon rate}

The Monte Carlo sample contains 30k events simulated with CORSIKA. In each event, cosmic particles are generated over an interval of $7.25 \mathrm{~ms}$, from $-4.05 \mathrm{~ms}$ to $3.20 \mathrm{~ms}$ with respect to the trigger time. This ensures complete coverage of the TPC readout window. The data sample contains $25 \mathrm{k}$ events. Both for data and $\mathrm{MC}$, the recorded readout length per event is to $-0.4 \mathrm{~ms}$ to $2.8 \mathrm{~ms}$. These samples correspond to $96 \mathrm{~s}$ and $78 \mathrm{~s}$ of cosmic exposure respectively, equivalent to approximately $3.5 \times 10^{5} \mathrm{TPC}$ muons.

The average number of reconstructed tracks $>25 \mathrm{~cm}$ per event in cosmic data and CORSIKA Monte Carlo is:

- CORSIKA MC: $15.09 \pm 0.05$ tracks per event. 
- Data: $15.30 \pm 0.03$ tracks per event.

Corresponding to a data/MC ratio of $1.014 \pm 0.004$. The systematic uncertainty will be discussed in Section 5.2.4.

\subsubsection{Reconstruction muon resolution and data/MC distributions}

The reconstructed muon-track length and angles are defined in Figure 5.5. Before the inTPC segment of the GEANT simulated muon trajectory can be compared with the reconstructed track, a space charge effect is applied on the simulated GEANT trajectory. This space charge effect - further described in Appendix B -is caused by the build-up of slowmoving positive ions in the TPC, leading to inhomogeneities in the electric field.
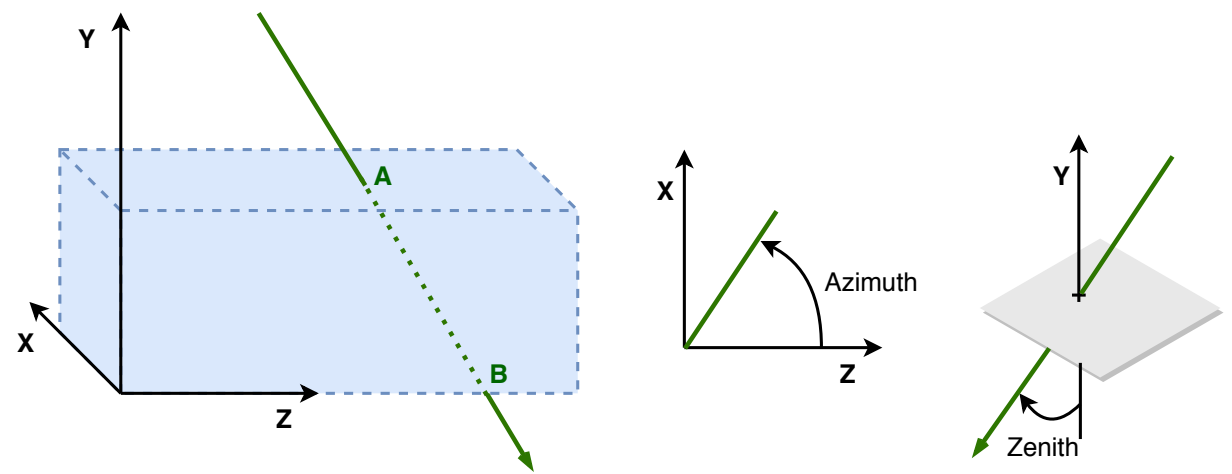

Figure 5.5: Schematic representation of a muon (green) crossing the MicroBooNE TPC. The trajectory inside the TPC is assumed to be a straight line. The muon enters the TPC at point $A$ and exits the TPC, or stops inside it, at point $B$. The track length, azimuth and zenith angles are obtained from the segment $A B$.

Reconstruction resolution The muon reconstruction resolution is determined by comparing the reconstructed quantities with the underlying simulated truth information. This is presented in the panels of Figure 5.6. Only tracks with a one-to-one correspondence to a CORSIKA simulated muon are used. As discussed previously, this includes $97.71 \%$ of the selected tracks. Figure 5.6 presents the reconstruction resolution obtained for the length and angles. 

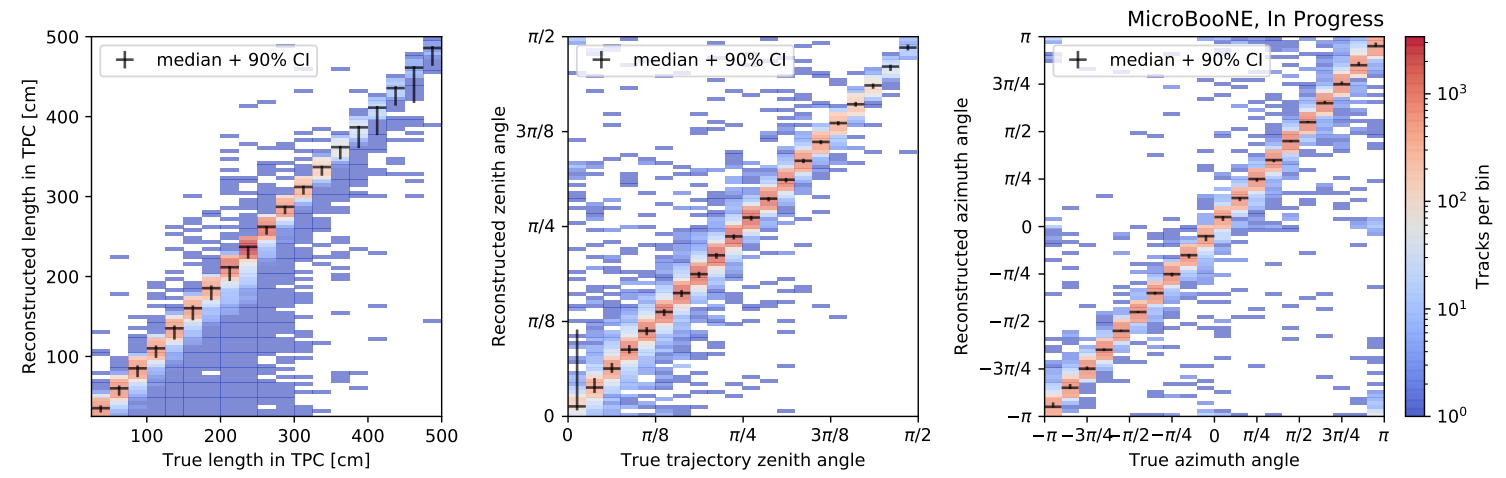

Figure 5.6: Reconstruction resolution for the track length (left), zenith angle (middle) and azimuthal angle (right). Space charge effect is applied on the truth variables in simulation before comparison with the reconstructed variables. The colour scale for the $2 D$ histograms is logarithmic. In black, the median of each bin with the $90 \%$ confidence interval are given.

The median and $90 \%$ confidence intervals are constructed in the following way (see the example in Figure 5.7):

1. In each bin of the truth variable (horizontal axis), the difference between the reconstructed and the truth values is calculated for each entry.

2. For every truth variable bin, the values corresponding to the 5th, 50th and 95th percentile are calculated in the reconstructed distribution.

3. The 50th percentile corresponds to the median and the 5th and 95th are respectively the lower and upper bounds of the $90 \%$ confidence interval for every truth variable bin.

Figure 5.8 shows the median and the width of the $68 \%$ and $90 \%$ confidence intervals for the simulated tracks. For the track length, the median indicates that reconstructed muon segments are slightly shorter than the true length. The bias ranges from $0.3 \mathrm{~cm}$ to $2.4 \mathrm{~cm}$ upwards shorter tracks, depending on the true length. The $90 \%$ confidence interval has a tail towards shorter tracks, due to reconstruction imperfections and unresponsive detector regions. The resolution, here defined as half the width of the interval, varies from $6 \mathrm{~cm}$, for short and vertically crossing tracks up to $\approx 15 \mathrm{~cm}$ for the longest tracks.

The middle and right panels of Figures 5.6 and 5.8 show the zenith and azimuth angles for 


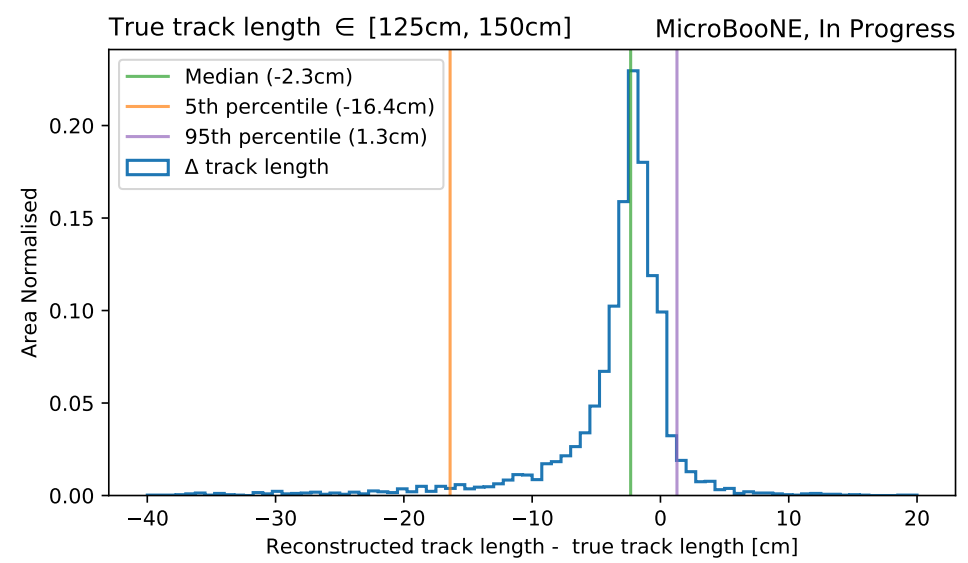

Figure 5.7: Example of how the reconstruction resolution is quantified, here for the track length, for tracks with a true length between $125 \mathrm{~cm}$ and $150 \mathrm{~cm}$. In blue, the histogram shows the difference between the true and reconstructed lengths. The median is given in green, the offset between the median and 0 is referred to as the bias. The 5th (orange) and 95th (purple) percentiles define a region that includes $90 \%$ of the tracks. The quoted resolution is half the width of this interval.

the muon segments. The bias of the median is below $0.1^{\circ}$ in both cases. The resolution of the zenith angle is better than $1^{\circ}$, except for the first two bins which have a tail towards larger zenith angles. These angles correspond to tracks parallel to the collection wire plane, creating a non-Gaussian hit on a single collection wire, leading to issues with the reconstruction. The resolution of the azimuthal angle varies between $0.8^{\circ}$ and $2.8^{\circ}$.

Data/MC distributions In Figures 5.9 to 5.11 , cosmic data is compared with the MC simulation. Two variants of the space charge effect are used in the simulated samples. In orange, a theoretically derived map is used, referred to as simulated space charge. In green, a datadriven approach is used, indicated as data-driven space charge in the legend. In red, the space charge effect is ignored, to create a sense of the magnitude and shape of its impact.

The data/MC comparisons for the three variables, including all reconstructed tracks longer than $25 \mathrm{~cm}$ show an overall deficit in Monte Carlo of $(1.44 \pm 0.37) \%$. The quoted error is statistical only and the systematic uncertainties will be added in Section 5.2.4.

The track length distribution in Figure 5.9 is peaked at $\approx 2.3 \mathrm{~m}$, this corresponds to the full 

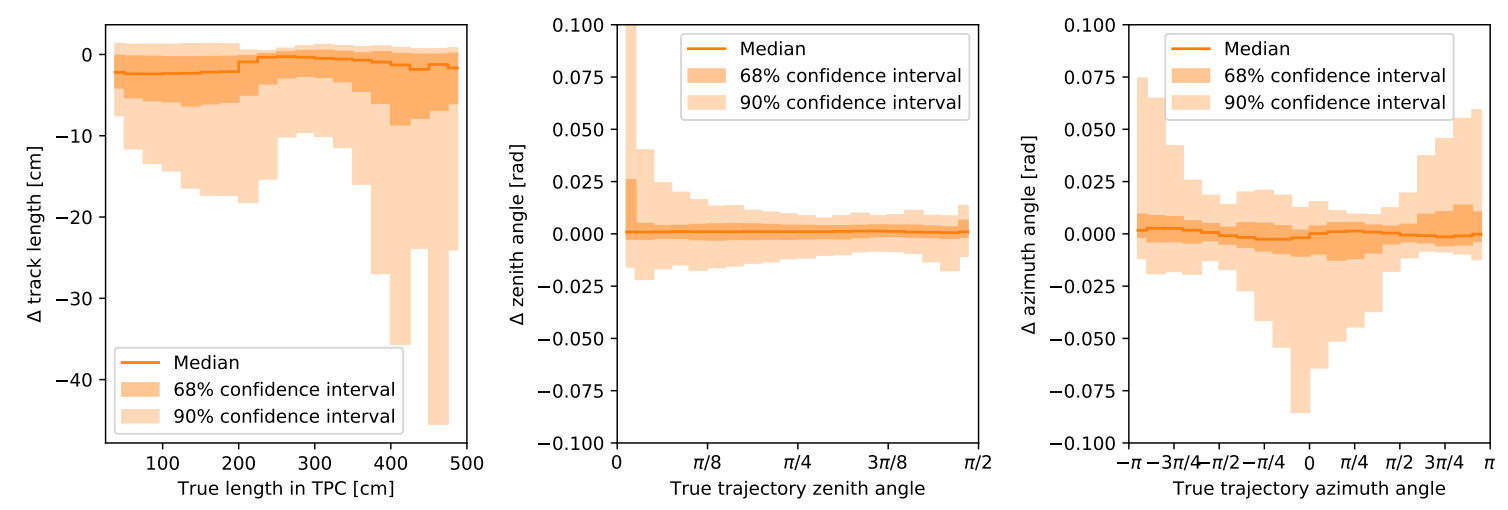

Figure 5.8: The bias, $68.3 \%(1 \sigma)$ and $90 \%$ confidence intervals, binned in the corresponding true quantities for the track length (left), zenith angle (middle) and azimuth angle (right). The resolution is defined here as half the width of the $90 \%$ confidence interval. It can be seen that the spread drastically decreases if a $68.3 \%$ is used. For long tracks, as can be seen in the left panel, the tails are poorly sampled due to limited statistics.

height of the TPC. The peak is widened towards shorter lengths due to the space charge effect which causes spacial distortions. The data/MC ratio for the track length is has two features:

1. The bump in the ratio between $2.0 \mathrm{~m}$ and $2.3 \mathrm{~m}$. The data in this region is situated between the two different space charge variants, indicating that the sample with theorybased space charge (green) exaggerates the effect slightly and the data with the datadriven model (orange) slightly underestimates it. This will be used in the next section to estimate a systematic uncertainty on the ratio.

2. The ratio rises slightly for reconstructed tracks $<50 \mathrm{~cm}$. The increased fraction of short tracks in data comes from broken tracks and tracks that are coming from particle types other than muons.

Although the generation of cosmic muons is flat in the azimuthal space, the cuboid shape of the MicroBooNE TPC shapes the observed distribution. This detector acceptance effect can be seen in Figure 5.10. The data/MC ratio is almost flat. Small features at $0^{\circ}$ and $180^{\circ}$ are explained by tracks parallel to the wire planes, where the drifted charge over the whole track length arrives coincidentally. Coherent noise removal affects the reconstruction for 


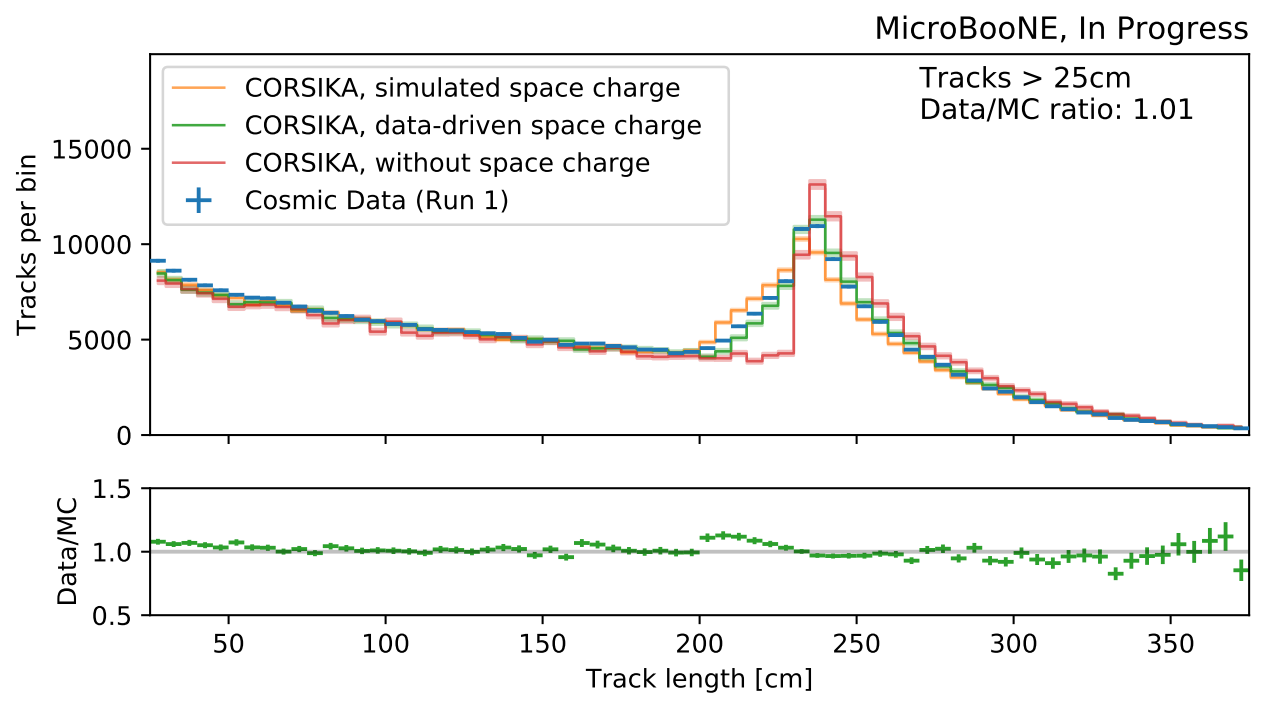

Figure 5.9: Comparison of the reconstructed track length in data (blue) and CORSIKA Monte Carlo, with theory-based (orange), with data-driven (green) and with no (red) space charge effect included. The bottom panel shows the data/MC ratio for the sample with datadriven space charge. The bin width on the horizontal axis is $5 \mathrm{~cm}$. A sharp turn on can be seen when no space charge is simulated (red) at $232 \mathrm{~cm}$, corresponding to the height of the detector. When the space charge effect is taken into account, the peak gets smeared out.

this topology, and the effect is included as a systematic error.

The comparison of the reconstructed zenith angle in data and MC is shown in Figure 5.11. It is worth noting that the zenith angle has an energy dependence due to the centre of the TPC being located $6 \mathrm{~m}$ underground. Tracks that traverse the TPC more horizontally (zenith angle approaching $\pi / 2$ ) therefore travel a further distance through the surrounding dirt. This is studied in the bottom panel of Figure 5.11. The bulk of the muons entering the TPC have an energy below $3 \mathrm{GeV}$, see also the left panel of Figure 5.4. For muons entering more horizontally, the median energy increases and can be as high as $20 \mathrm{GeV}$. Due to the limited size of the plane where the muons are generated at $18 \mathrm{~m}$ above the TPC, the simulation is not fully covering the zenith phase space. More precisely, from 75 degrees, the Monte Carlo under predicts the horizontal muon flux. Due to the very low flux reaching the TPC at these angles, the effect on the integrated muon flux is small but included as a systematic error in the next section. The energy threshold is further discussed in Appendix C.3. 
MicroBooNE, In Progress

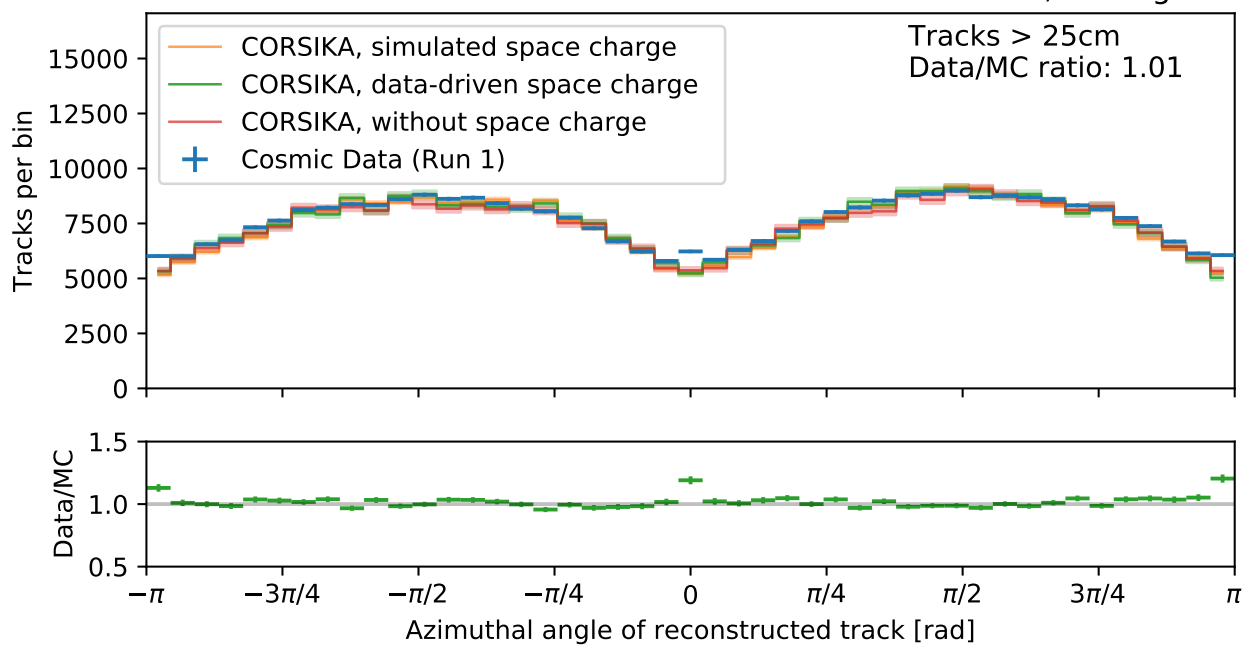

Figure 5.10: Comparison of the reconstructed track azimuth angle in data (blue) and CORSIKA Monte Carlo, with theory-based (orange), with data-driven (green) and with no (red) space charge effect included. The bottom panel shows the data/MC ratio for the sample with data-driven space charge. The bin width on the horizontal axis is $8^{\circ}$ 

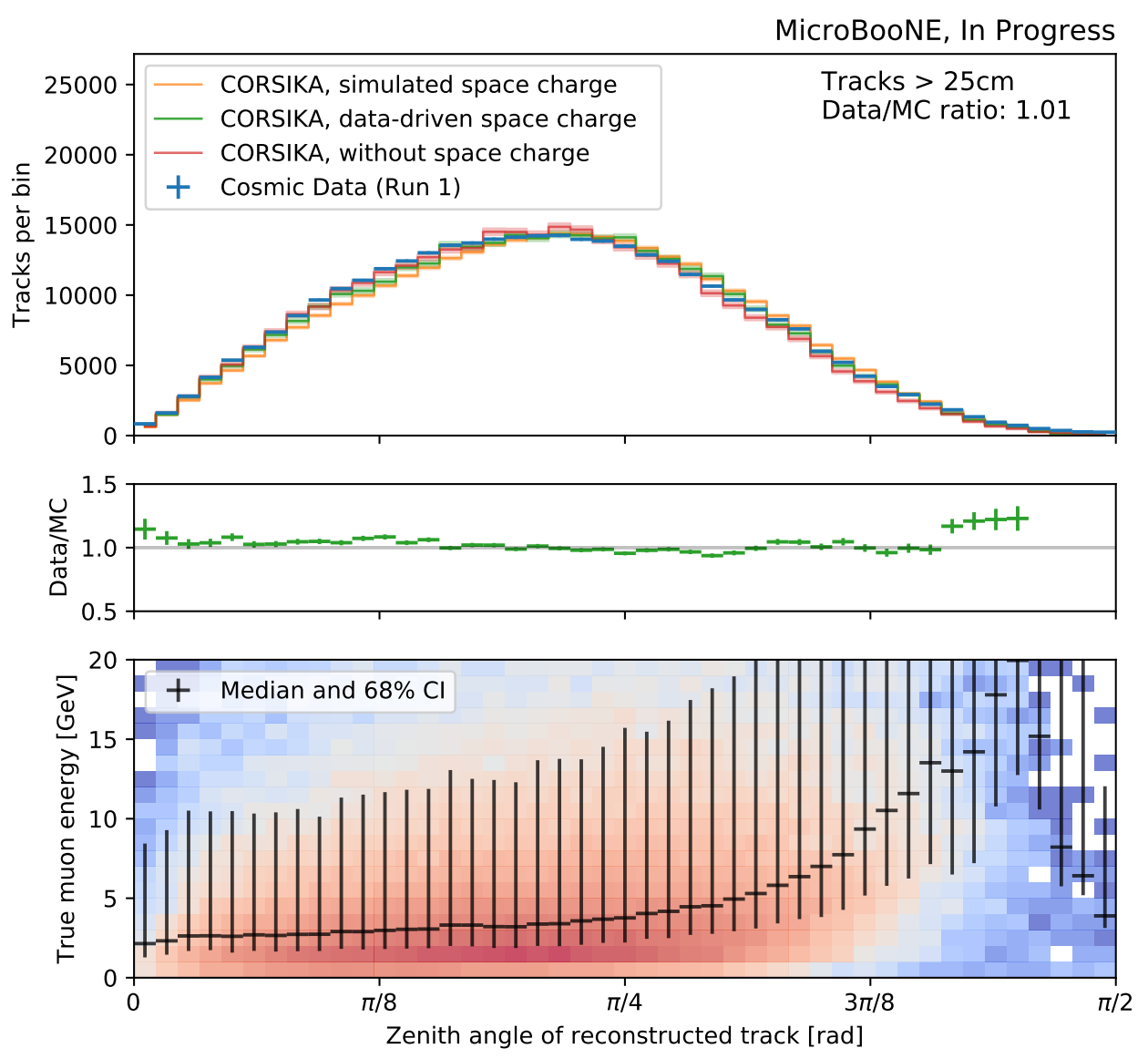

Figure 5.11: Comparison of the reconstructed track zenith angle in data (blue) and CORSIKA Monte Carlo, with theory-based (orange), with data-driven (green) and with no (red) space charge effect included. The middle panel shows the data/MC ratio for the sample with data-driven space charge. The $2 D$ histogram at the bottom shows the true muon energy for the data-driven space charge sample. The black line indicates the median true energy and the error bars correspond to the 16th and 85th percentile, covering a $68.3 \%$ interval. The bin width on the horizontal axis is $2^{\circ}$. Although the full phase space from $0^{\circ}$ to $90^{\circ}$ is given, the simulation significantly undercovers the horizontally oriented tracks $-75^{\circ}$ and higher - due to the finite extent of the simulation. See Section 5.2.4 for a further discussion. 


\subsubsection{Systematic error estimation}

As given in Section 5.2.2, the data/MC ratio using tracks above $25 \mathrm{~cm}$, including the statistical error is: $1.014 \pm 0.004$. Four sources of systematic uncertainties that impact the ratio are treated here and will be combined afterwards.

1. Incomplete angular coverage. Figures 5.10 and 5.11 show, limitations in the simulation that lead to data/MC discrepancies in small corners of the phase space. The ratio is calculated excluding those regions, the difference between the ratios with and without the regions is taken as this systematic uncertainty.

- Zenith angle. When the zenith angle is restricted between 3 and 70 degrees, the ratio becomes 1.011 .

- Azimuth angle. Around 0 and 180 degrees - where the tracks are parallel to the collection plane - a region of 4 degrees is excluded, the ratio becomes 1.008.

2. Length criterion. In these results, tracks above $25 \mathrm{~cm}$ are required as a higher purity can be achieved by increasing the track length. In Figure 5.12, the purity and data/MC ratio is evaluated for lengths ranging from $5 \mathrm{~cm}$ to $175 \mathrm{~cm}$.

3. Space charge effect. As demonstrated in Figure 5.9, the modelling of the space charge effect can affect the length of reconstructed tracks. The stronger the effect the more tracks get shortened in the reconstruction. In Figure 5.12, the data is compared with a sample using a theory-drive simulated space charge model, a sample using a databased approximated and a sample where space charge was not included in the simulation at all.

4. LArTF building geometry. The concrete walls, roof and the building geometry can impact the simulated cosmic rate in the TPC. The potential effect is estimated by varying the concrete density of the walls and roof by $+30 \%$ (increased LArTF building) and $-30 \%$ (reduced LArTF building).

The left panel of Figure 5.12 shows the purity as a function of the length criterion. The difference between the chosen space charge simulation or LArTF building model does not 

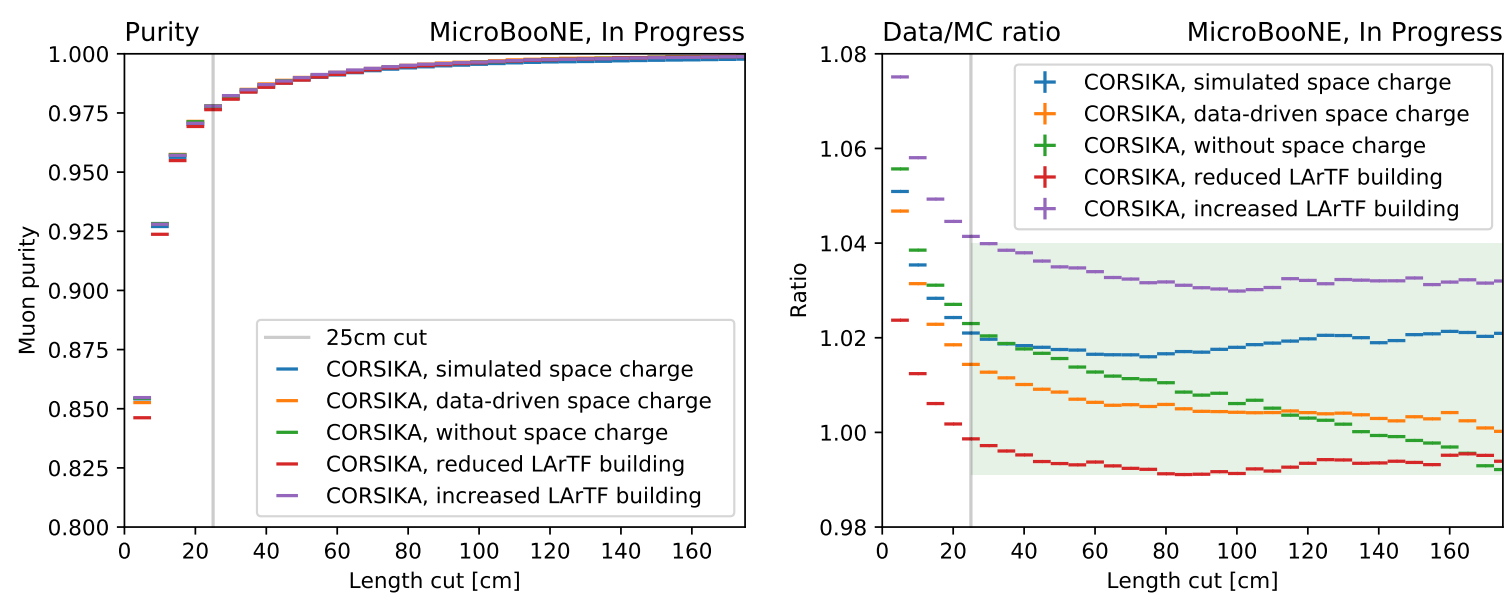

Figure 5.12: Dependence of the reconstructed track muon purity and the data/MC ratio on the length criterion. The purity is defined as the fraction of tracks that is uniquely matched to a simulated cosmic muon. In orange, the purity and ratio is given using the CORSIKA sample with data-driven space charge effect included. In blue, a CORSIKA sample where the theory-based space charge simulation is used. In green, a sample without simulated space charge is used. The purple and red curves correspond to samples with data-driven space charge effect where the concrete density of the LArTF building is varied with $\pm 30 \%$. Both LArTF building variations use the data-driven space charge model. The green band represents the maximum variation including systematic effects of the different samples for tracks with a reconstructed length above $25 \mathrm{~cm}$.

significantly impact the purity. By looking at longer lengths, the purity can be further increased. The right panel shows the effect of the length criterion on the data/MC ratio. The data corresponds best with a space charge effect between the theory-based and the datadriven simulated samples. It can be seen that the LArTF geometry uncertainty dominated the uncertainty with a magnitude of $2 \%$, as discussed in Section 5.1.3. The combined systematic error, including the variation in the length criterion, the building geometry and the space charge effect, is taken to be the extreme values for the ratio using a length criterion ranging from $25 \mathrm{~cm}$ to $175 \mathrm{~cm}$, as defined by the green box in the left panel of Figure 5.12. A data/MC ratio of $1.014 \pm 0.004$ (stat.) ${ }_{-0.022}^{+0.031}$ (syst.) is found.

The systematic uncertainty derived from Figure 5.12 is now combined in quadrature with the systematic uncertainty originating from the angular dependency:

$$
1.014 \pm 0.004 \text { (stat.) }{ }_{-0.023}^{+0.031} \text { (syst.) }
$$




\subsection{PMT-based muon rate measurement}

In this section, the muon rate is measured using MicroBooNE's optical system, consisting of 32 8-inch PMTs, introduced in Section 3.2.2. The rate of optically reconstructed signals can be related to the cosmic muon-rate, cross checking the TPC-based result.

Cosmic discriminator flashes The flashes used for the cosmic measurement presented here are reconstructed during the readout window of $4.8 \mathrm{~ms}$ per event, excluding the beam discriminator window of $23.45 \mu$ s (see Section 4.1.2). A threshold of 8 Photo-Electrons (PE) per PMT is required, and at least two PMTs above threshold are needed to create a flash. When a flash is created, all PMT signals above threshold are summed for $8 \mu \mathrm{s}$, and this sum is required to be at least $30 \mathrm{PE}$. The time of the flash corresponds to the rising edge time of the scintillation light and is usually about $60 \mathrm{~ns}$ delayed due to the shaper time as discussed in Section 3.2.2. The $8 \mu$ s integration window is chosen to capture the light created by the slow component of the liquid argon scintillation light, which has a decay constant of $1.6 \mu \mathrm{s}$. During this integration window, no additional flash can be created.

\subsubsection{Purity}

Simulated primary CORSIKA particles are matched to reconstructed flashes using time. More precisely if a flash is reconstructed with a delay between $50 \mathrm{~ns}$ and $500 \mathrm{~ns}$ compared with the CORSIKA particle generation time, it is attributed to that simulated primary particle. If only TPC-crossing muons are used to match the reconstructed flashes, $(81.6 \pm 0.1) \%$ of the flashes are matched. Including all primary CORSIKA particles, this increases to $(97.1 \pm 0.1) \%$.

The $97.1 \%$ can be split up in to particles crossing the active TPC (83.1\%) and particles the majority muons - crossing the cryostat without entering the active TPC $(14.0 \%)^{2}$. The $2.9 \%$ of unmatched flashes can be attributed to TPC interactions that are more than $500 \mathrm{~ns}$ delayed compared to the CORSIKA primary particle and late light re-flashing, typically

\footnotetext{
${ }^{2}$ Figure 3.6 illustrates the location of the TPC inside the cryostat
} 
with a low photo-electron intensity.

$94.1 \%$ of the $97.1 \%$ of matched flashes is matched to muons, both inside and outside the TPC. The remaining fraction is caused by inelastic neutron scattering $(2.1 \%)$ and the remaining $0.9 \%$ corresponds to $\gamma$, proton and $e^{+/-}$interactions.
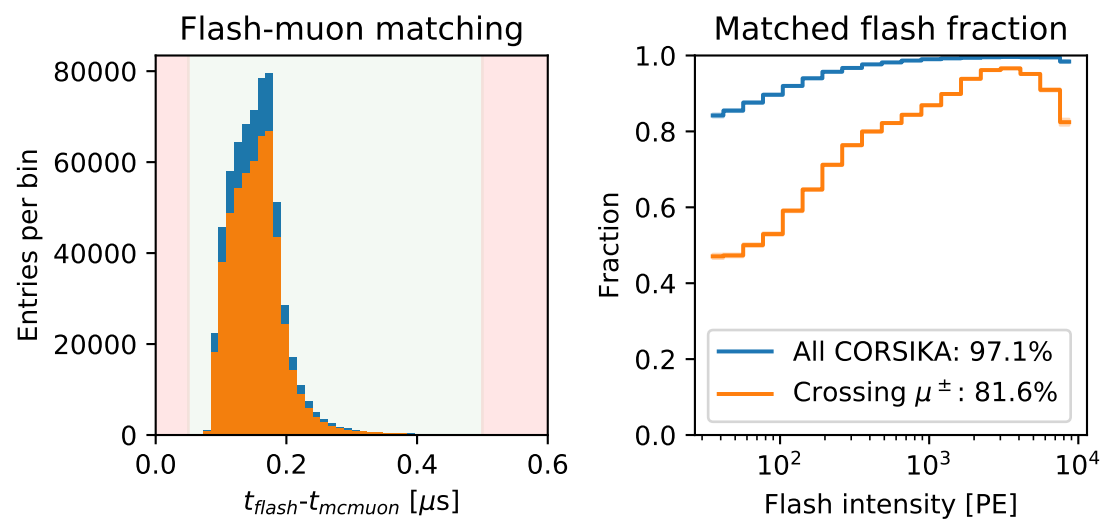

Figure 5.13: (Left) Histogram of time differences between the generated particle and the closest delayed reconstructed flash. In orange, only CORSIKA muons in the TPC are used. In blue, all CORSIKA primary particles are used to explain reconstructed flashes. The shaded green area is the time differences in which matching is performed. Random coincidences would show up as a flat contribution but are negligible. (Right) Fraction of flashes that is matched to a primary particle as a function of the flash intensity. As seen from the blue curve in the right panel, above $\approx 200 \mathrm{PE}$, a perfect correspondence between flashes and CORSIKA simulated primary particles is found.

\subsubsection{Efficiency}

Of the simulated muons in the TPC, $(81.3 \pm 0.3) \%$ correspond to a reconstructed flash. Part of the inefficiency can be explained by two effects that cause dead time inside the $4.8 \mathrm{~ms}$ window:

- There is a $23.45 \mu$ s beam discriminator window, during which no cosmic flashes are constructed.

- For every reconstructed optical flash, a dead time of $8 \mu$ s occurs to prevent the slow light component to create new spurious flashes. 
Therefore, the dead time per event, $\tau_{\text {dead_event }}$ is:

$$
\tau_{\text {dead_event }}=(23.45 \mu \mathrm{s}+(\text { avg. number of flahses per event }) \times 8 \mu \mathrm{s}) / 4.8 \mathrm{~ms} .
$$

In Monte Carlo, there are $\approx 26$ cosmic flashes per event. Combining this with the beam discriminator window, $4.8 \%$ of the generated muons are not expected to create a flash.

Figure 5.14 illustrates how the flash efficiency is further reduced for muons crossing at high $x$, far from the PMTs. A similar, although less pronounced, effect is found in the $z$-direction (see Figures C.3 and C.4). A subsample of CORSIKA crossing muons can be constructed, requiring that the average $x$ position is at least half a meter from the cathode and the average $z$ position is at least $50 \mathrm{~cm}$ from the edges. In this sample - aimed to reduce the locationdependent inefficiencies - $(94.4 \pm 0.3) \%$ of muons create a flash object. Due to dead time, as discussed before, an inefficiency of $4.8 \%$ is expected. The remaining inefficiency of less than one percent is explained by muons with a short length $(\mathcal{O}(\mathrm{cm}))$ inside the active TPC volume and/or a low energy $(\mathcal{O}(\mathrm{MeV}))$ at the point of entering the TPC. These effects are quantified in Appendix C.3.
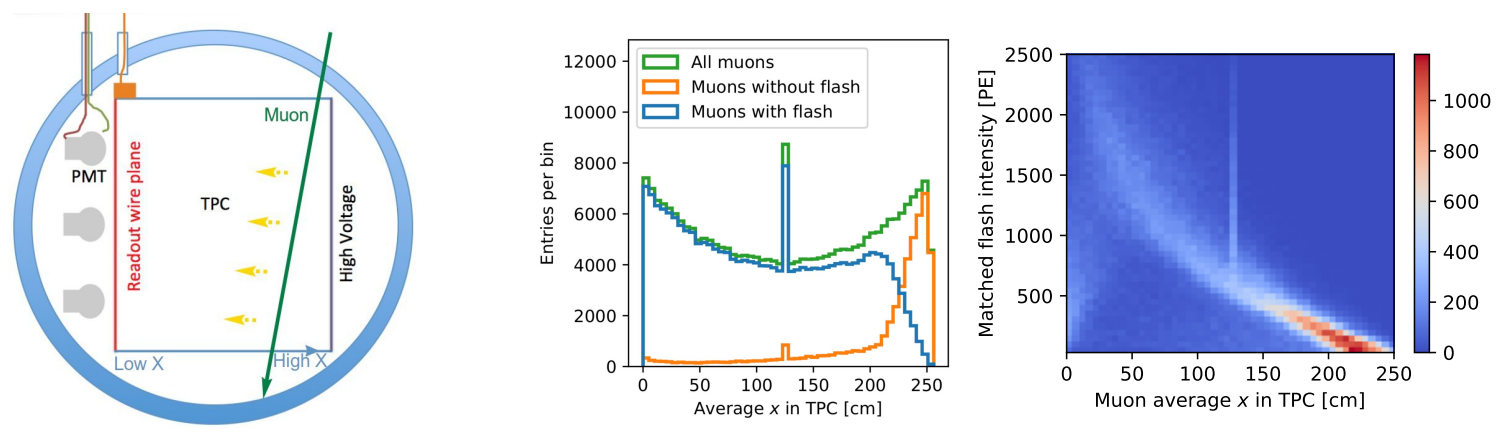

Figure 5.14: (Left) Schematic side view of the MicroBooNE cryostat, showing a muon crossing at high $x$. (Middle) Average $x$ position of the crossing muon trajectory inside the active TPC volume. The crossing muons (green) are split up in those that have a corresponding reconstructed flash $(81.3 \%$, blue) and those that do not $(18.7 \%$, orange). The central peak corresponds to anode-cathode crossing muons. Note the sharp decline for tracks situated within half a meter from the cathode plane. (Right) The dependence of the flash intensity as a function of the average distance from the wire planes. Far from the cathode, a clear low intensity peak is visible, responsible for the unmatched muons. 


\subsubsection{Rate comparison in data and Monte Carlo}

The retrieved flash rates, with statistical errors, are:

- Monte Carlo: $(4.472 \pm 0.011) \mathrm{kHz}$

- Data: $(4.427 \pm 0.007) \mathrm{kHz}$

This rate in both MC and data is affected by the dead time introduced by each flash of $8 \mu \mathrm{s}$, which can be corrected for in the following way:

$$
R_{\text {measured }}=R_{\text {true }} \int_{8 \mu \mathrm{s}}^{\infty} e^{-t \cdot R_{\text {true }}} \mathrm{d} t \approx 0.96 \cdot R_{\text {true }}
$$

Here, $R_{\text {true }}$ is the flash rate corrected for dead time. The magnitude of the correction is approximately $4 \%$ and results in the following dead-time-corrected flash rates:

- Monte Carlo: $(4.642 \pm 0.011) \mathrm{kHz}$

- Data: $(4.593 \pm 0.007) \mathrm{kHz}$

An excess in Monte Carlo over data of $(1.0 \pm 0.3) \%$ is observed. The ratio is found to be stable over a wide range of flash intensities.

\subsubsection{Systematic error estimation}

The sources of systematic error can be split up into light modelling systematic uncertainties and the uncertainty of the LArTF geometry.

Flash photo-electron threshold Flashes are created with a minimum of 30 photo-electrons, this threshold can be changed to study the sensitivity of the ratio due to the very dimmest flashes. After doubling the threshold, the ratio changes from 0.990 to 0.992 , compatible with a statistical fluctuation.

Light yield variations Over different data taking periods, MicroBooNE experienced a decreasing light yield in data (see Figure 3.12). This mostly happened after the first run of 
data-taking, which is used in this section. Nevertheless, the impact of changing light yield is evaluated by varying the total flash PE with $\pm 30 \%$ in Monte Carlo. The decreased scintillation light production leads to a ratio of 1.006 and increased the light yield leads to a ratio of 0.974 . Therefore this uncertainty is symmetric and affects the result by $1.6 \%$.

Out-of-TPC muon flashes The modelling of light propagation from outside the TPC towards the PMTs could affect the result. As was discussed in Section 5.3.2, a significant fraction of the flashes are caused by muons crossing the cryostat without entering the TPC. Their contribution can be studied by including only flashes which are centred inside a restrained fiducial region on the YZ-plane.

In Figure 5.15, the contribution of light originating outside the TPC is reduced by looking at flashes centred at least one meter away from the edges in the $z$-direction and below $30 \mathrm{~cm}$ in the vertical direction. After applying this selection, the fraction of flashes caused by muons entering the TPC increases from $81.6 \%$ to $87.5 \%$ while the contribution of flashes caused by muons in the cryostat without entering the TPC decreases from $12.6 \%$ to $6.2 \%$. The data/MC ratio changes from 0.990 to 0.992 . The systematic uncertainty is taken to be twice the difference in the ratio to account for the remaining contribution of flashes caused by muons outside the TPC.

LArTF geometry modelling This uncertainty is analogous to the one discussed for the TPCbased cosmic rate measurement. The two samples, one with increased and one with decreased wall/roof concrete density, are used to estimate the effect on the flash rates. It is found that increasing the concrete density by $30 \%$ leads to a reduction in flash-rate of $2.7 \%$, while decreasing it with $30 \%$ increases the flash-rate by $2.3 \%$.

Combining the statistical and systematic uncertainties The different sources of systematic uncertainty are summed in quadrature. This leads to a data/MC ratio of $0.990 \pm 0.003$ (stat.) ${ }_{-0.028}^{+0.032}$ (syst.) 

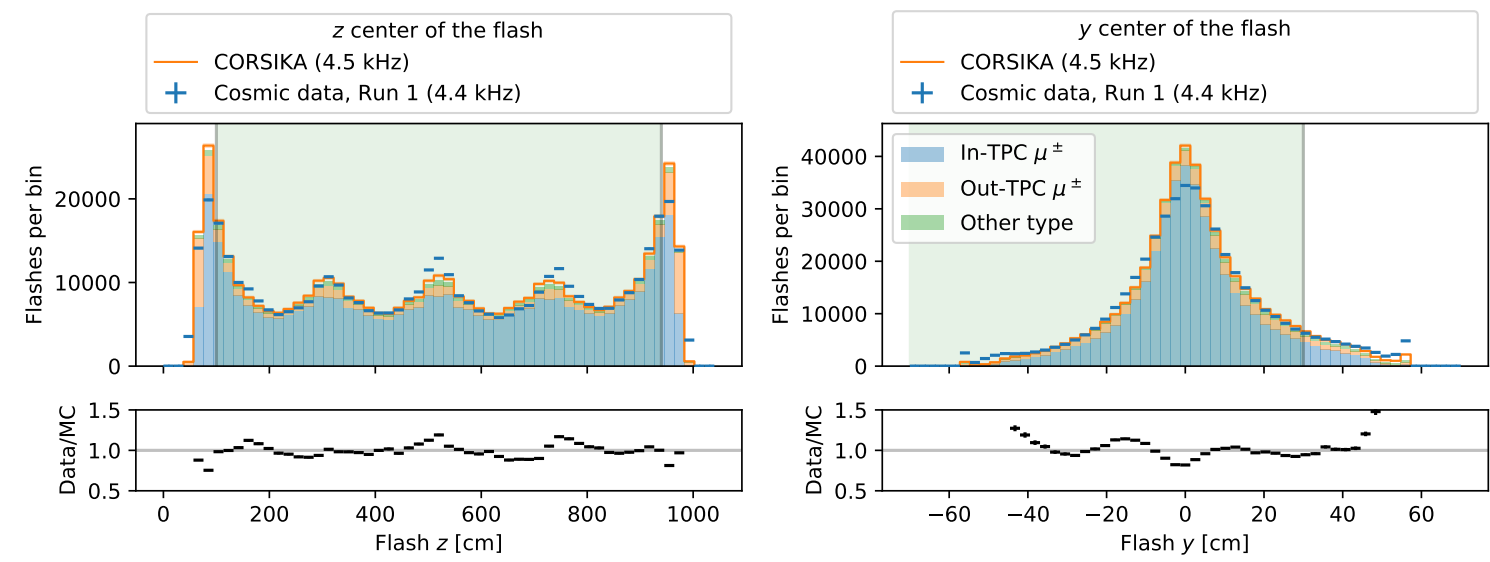

Figure 5.15: Position of the centre of the flash in the $z$ (left) and $y$ (right) directions. The flashes are identified as caused by muons entering the TPC (blue), muons entering the cryostat but not the TPC (orange) and a small contribution of other CORSIKA primary particles (green). The shaded green area is a restricted area where the contribution of in-TPC muons is more dominant and the fraction of light coming from outside the TPC is reduced. The bumped structure is explained by the physical position of the PMTs, as shown in Figure 4.2. 


\subsection{Towards a CRT-based muon rate measurement}
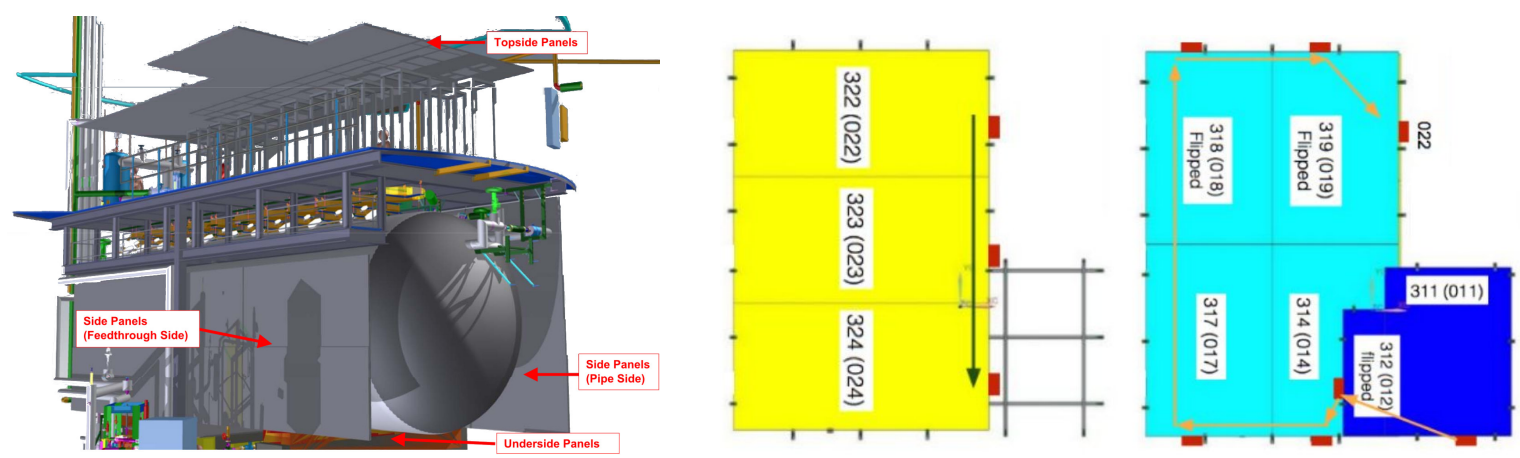

Figure 5.16: (Left) Rendering of MicroBooNE showing the CRT panels partly covering four sides of the cryostat. (Right) The bottom CRT panels are located $1.4 \mathrm{~m}$ under the TPC and are composed of modules arranged in three layers (yellow, cyan and blue) with 9 modules in total. Each module consists of two layers of strips, oriented perpendicular to each other. Figure from [52].

The MicroBooNE Cosmic Ray Tagger (CRT) system is an external sub-system designed to improve identification and rejection of cosmic muons [53]. The system consists of 73 scintillating modules made of interleaved layers of plastic scintillating strips situated on the top, bottom, and two sides parallel to the neutrino beam (Section 3.2.3 and left panel Figure 5.16). Scintillation photons induced by charged particles traversing the layers of each module are collected by silicon photo-multipliers at the end of the strips. Using these scintillator strip signals, the geometric positions and time of traversing cosmogenic muons can be reconstructed. In this preliminary study, only the bottom ${ }^{3}$ panel is used, sketched in Figure 5.16.

An area of $13 \mathrm{~m}^{2}$, indicated on Figure 5.17, is selected to measure the through-going muon rate in data and Monte-Carlo. Within a CRT module, a CRT hit is created for each and every pair of perpendicular oriented strips with hits coincident in time. To convert the hit rate to physical activity in the CRT, two effects need to be taken into account:

1. Coincidental hits. When a muon enters the CRT module under an angle, or if scintillator light leaks from one scintillator bar into others, multiple CRT hits will be created.

\footnotetext{
${ }^{3}$ Due to the limited physical extend of the CORSIKA simulation, the other panels are not fully covered.
} 

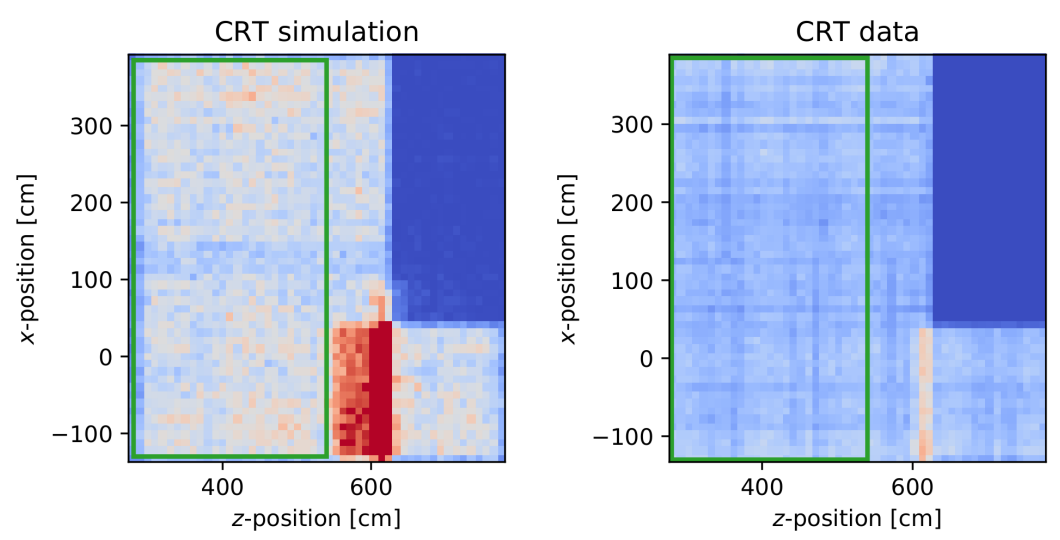

Figure 5.17: Two dimensional hit density of the CRT bottom panel in simulation (left) and data (right), uncorrected for coincidental hits and dead time. The simulation has a higher CRT hit rate than seen in data. The hot spot on the bottom occurs where multiple modules are overlapping and is excluded for this study. The green rectangle $\left(13 \mathrm{~m}^{2}\right)$ indicates the area that was used for this measurement.

These hits are within $\mathcal{O}(100 \mathrm{~ns})$ from each other.

2. Dead time. Each module has a front-end board with a dead time of $2.2 \mu \mathrm{s}$.

The rate of CRT-crossing particles can be obtained from the time between CRT hits. Assuming that the frequency of CRT crossings follows a Poisson distribution, the intervals between them are expected to be distributed exponentially, which is demonstrated to largely be the case in Figure 5.18. If the time between successive hits is short, $<\mathcal{O}(25 \mu \mathrm{s})$, this assumption breaks down. At these time scales, coincident hits and dead-time become important. Additionally, particle interactions in the atmosphere or CRT surroundings might result in particle avalanches and correlated hits. The tails of the distributions are fitted with an exponential function to obtain the rate of independent CRT events in the selected area of the bottom panel:

- CRT simulation activity rate through bottom panel: $(92.80 \pm 0.25) \mathrm{Hz} / \mathrm{m}^{2}$

- CRT data activity rate through bottom panel: $(89.3 \pm 0.3) \mathrm{Hz} / \mathrm{m}^{2}$ resulting in data/MC ratio of $0.963 \pm 0.006$ (stat.). This measurement is preliminary and is currently being followed-up to pin down the efficiency and purity of the CRT activity. 

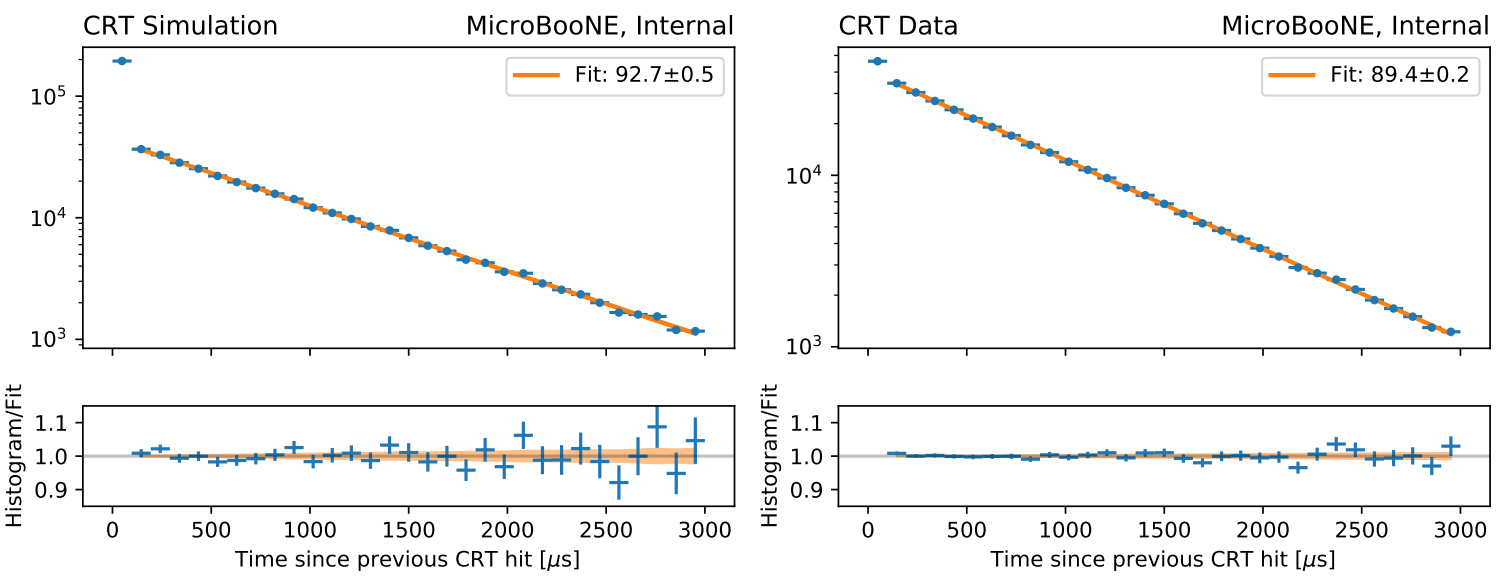

Figure 5.18: Time differences between sequential CRT hits and exponential fit (orange) in the range $50 \mu$ s to $3000 \mu$ s to obtain the particle rate of CRT activity. The excess in the first bin is due to coincidental hits in neighbouring CRT strips. As discussed in the main text, due to dead time, correlations and multiple strip crossings, the coincidental hits are currently poorly modelled and excluded from the fit.

These might lead to corrections in the CRT simulation and affect the quoted data/MC ratio. Additionally, the systematic uncertainties connected to the CRT are also being studied by the collaboration. This work serves currently as the first validation of the CRT data and simulation. In Chapter 6, a set of CRT-based techniques will be introduced to remove cosmogenic backgrounds in neutrino event selections. 


\subsection{Results \& discussion}

In the previous sections, ratios between muon-related activity in data and Monte Carlo were obtained. These can be equivalently stated as a muon flux per area at the position of the TPC. To obtain this rate, the data/MC ratio is scaled to the simulated rate. It is important to note that this rate does not depend on the systematic uncertainty related to the LArTF geometry. If, for example, one takes a simulated geometry including additional shielding, the obtained ratio would be higher. To calculate the muon rate at the TPC this increase cancels out by scaling the ratio to the prediction obtained from the simulation. The cosmic muon rate obtained independently with the TPC (Section 5.2) and the PMTs (Section 5.3) is quoted with the systematic uncertainty in the first column of Table 5.2. Excluding the systematic uncertainty related to the LArTF geometry, the two measurements and their systematic uncertainties are uncorrelated. The rates are in agreement within the one sigma range.

Relying on the accuracy of the LArTF building geometry included in the GEANT simulation, the ratios can be converted into a muon rate above the roof of the experiment hall. The latter being more useful for other Fermilab experiments. This is done and compared with the CORSIKA rates in the last column of Table 5.2. In this case, the dominant systematic uncertainty is due to the LArTF building geometry and is fully correlated between the two measurements. The CRT measurement is not included due to the preliminary status of the CRT simulation.

Comparison with other experiments Figure 5.19 compares the value for the integrated muon flux obtained with the MicroBooNE detector with other measurements. This and previous measurements indicate a clear preference of the CORSIKA prediction of $(127.7 \pm 0.2) \mathrm{Hz} / \mathrm{m}^{2}$ to the CORSIKA+CMC prediction of $(160.9 \pm 0.3) \mathrm{Hz} / \mathrm{m}^{2}$. The MicroBooNE measurement using the TPC and the PMT are in good agreement with each other and the CORSIKA prediction. 
Table 5.2: Summary of the muon rates in simulation and data, at the level of the TPC entrance and above the roof of the LArTF building. The TPC and PMT data rate is shown, as calculated in Sections 5.2 and 5.3 respectively. The simulation rates for the two CORSIKA models are presented. The default model uses only protons as cosmic particles, as described in Section 5.1. The alternate model, using the CMC is also given for comparison.

\begin{tabular}{llll}
\hline & & Rate at TPC $\left(\mathrm{Hz} / \mathrm{m}^{2}\right)$ & Rate above roof $\left(\mathrm{Hz} / \mathrm{m}^{2}\right)$ \\
\hline \multirow{2}{*}{ MC } & CORSIKA default & $111.6 \pm 0.3$ & $127.7 \pm 0.2$ \\
& CORSIKA + CMC & $140.1 \pm 0.3$ & $160.9 \pm 0.3$ \\
\multirow{2}{*}{ Data } & TPC & $113.2 \pm 0.5$ (stat.) ${ }_{-1.6}^{+1.1}$ (syst.) & $129.5 \pm 0.5$ (stat.) ${ }_{-3.0}^{+4.0}$ (syst.) \\
& PMT & $110.4 \pm 0.4$ (stat.) ${ }_{-2.0}^{+1.8}$ (syst.) & $126.5 \pm 0.4$ (stat.) ${ }_{-3.5}^{+4.1}$ (syst.) \\
\hline
\end{tabular}

Seasonal variation If the temperature of the atmosphere changes by $\Delta T_{\text {eff }}$, the muon flux - $I_{\mu}$ - will change by $\Delta I_{\mu}$. The variation of muon rate is related to the effective temperature of the atmosphere as [85]:

$$
\frac{\Delta I_{\mu}}{I_{\mu}}\left(E_{\mu}\right)=\alpha_{T}\left(E_{\mu}\right) \frac{\Delta T_{e f f}}{T_{e f f}} .
$$

Here, the coefficient $\alpha_{T}$ depends on the energy of the muons. Incoming cosmic particles, mainly protons, will interact with the atmosphere to produce kaons and pions. There are two competing effects that alter the muon flux:

1. When the temperature increases, the density of the atmosphere decreases and the interaction probability for kaons and pions decreases, increasing the chance that those particles will decay into muons.

2. In summer, the less dense atmosphere leads to interactions happening at higher altitude. The chance of muons decaying before reaching the detector is thus increased. This effect is especially important for low energy muons.

Figure 5.20 shows the value of $\alpha_{T}$ at different detector depths, measured by different experiments up to 2012. The MicroBooNE detector has a water equivalent depth of close to $0 \mathrm{~m}$, where the value of $\alpha_{T}$ approaches 0 . Furthermore, the data sample used throughout this note spans a period between February and May 2016, and is therefore not suited to measure the seasonal variation in the cosmic-ray muon flux. 


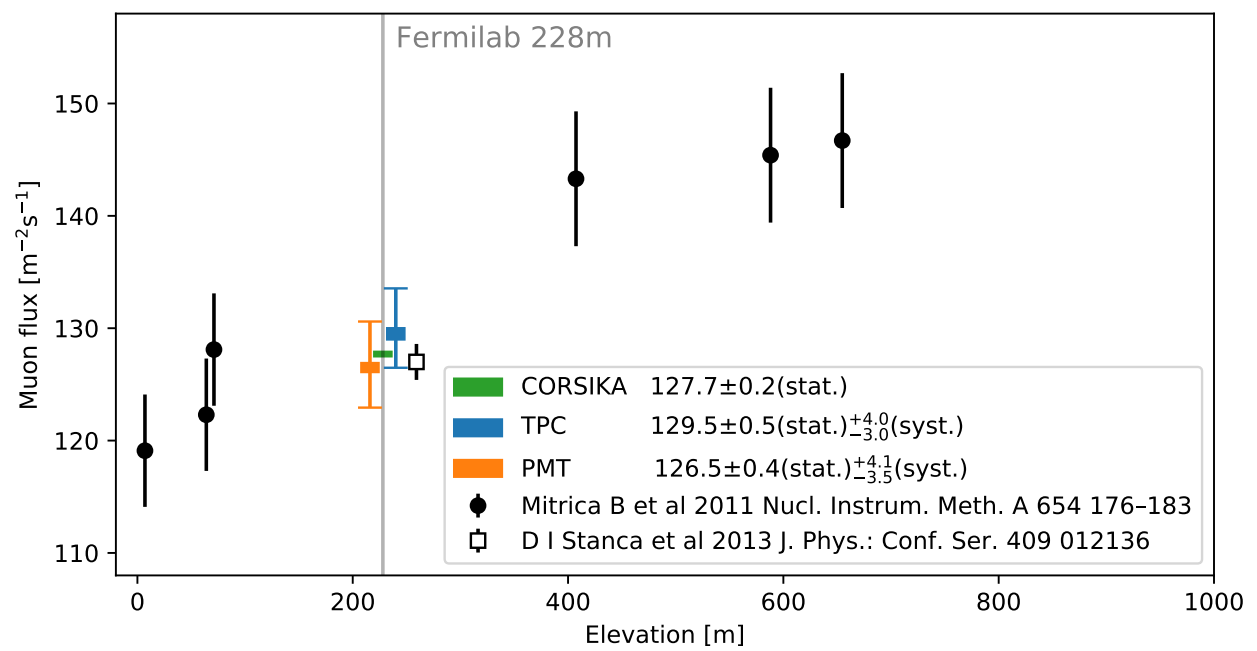

Figure 5.19: Comparison of the integrated muon flux measurement in this work with previous work $[83,84]$ (black), for different elevations. The vertical grey line indicates the altitude of Fermilab. The TPC (blue) and PMT (orange) measurements are added and compared with the CORSIKA(FLUKA) prediction (green). The vertical width of the data points represents the statistical error only, while the error bars represent the combined stat. $\oplus$ syst. errors. The blue and orange points are horizontally offset from the grey line for visual clarity.

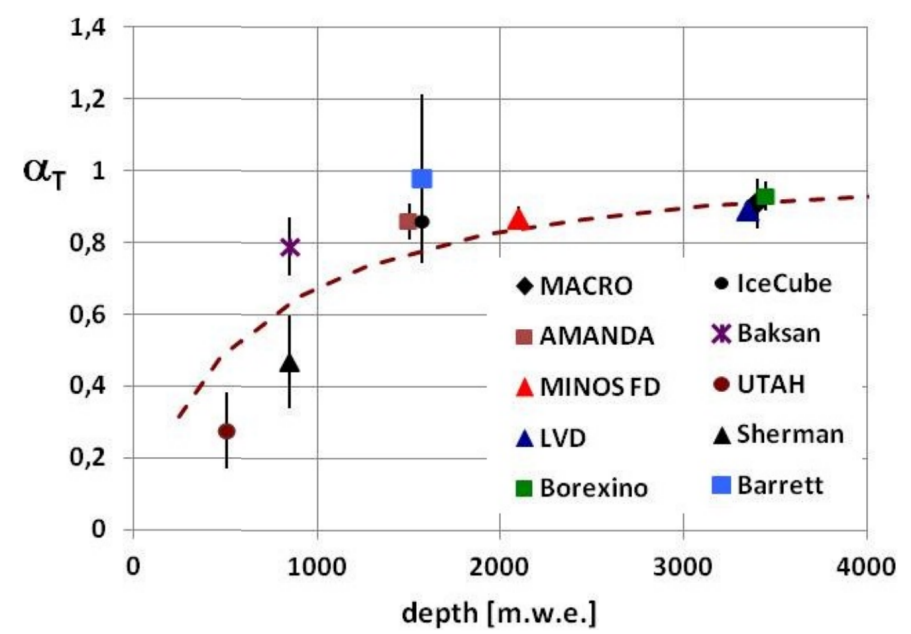

Figure 5.20: The temperature coefficient $\alpha_{T}$ as a function of detector depth. The dashed curve is the prediction using the pion-only model (Ambrosio et al., 1997c). The points correspond (from top to bottom): Ambrosio et al. (1997c), Bouchta (1999), Adamson et al. (2010), Selvi (2009), Bellini (2012), Desiati (2011), Andreyev et al. (1991), Cutler et al. (1981), Sherman (1954), Barrett et al. (1954). Figure from [85]. 


\section{6}

\section{A Flavour Agnostic Neutrino Selection}

The work presented in this thesis relies on the Pandora approach for event reconstruction, described in Section 4.2.3. The scope of Pandora is to do the low-level pattern-recognition steps of the reconstruction, i.e. group hits into clusters, clusters into particles and particles into hierarchies. This chapter focuses on the cosmic-rejection aspect of the analysis workflow which leads to the identification of neutrino interactions. The identification of neutrino interactions is performed with the aim to keep the bias towards the lepton flavour and interaction type as minimal as feasible, i.e. flavour agnostic. Pandora's pattern-recognition output is combined with scintillation light information to isolate candidate neutrino interactions. Moreover, it is described how the additional information from the Cosmic Ray Tagger (CRT) can improve cosmic rejection. The collection of tools described in this chapter is called the NeutrinoID and forms the cornerstone of most neutrino analyses in the 
MicroBooNE collaboration. Two immediate examples will be introduced in Chapter 7 - a charged-current muon-neutrino selection - and in Chapter 8 - a charged-current electronneutrino selection. At the beginning of this chapter - Section 6.1 - two signal definitions are introduced which will be used to benchmark the performance of the NeutrinoID. Furthermore, the normalisation of the data and the simulation is discussed, which will then be used throughout the rest of this work.

\subsection{Signal definition \& normalisation}

Signal Definition The selection developed here will be bench-marked using two signal definitions, targeting charged-current neutrino interactions in both the muon and the electron flavours. For an event to be considered signal, there are two requirements that are applied at truth level:

1. The event contains a simulated charged-current neutrino interaction and has a final state with lepton with a true kinetic energy of at least $20 \mathrm{MeV}$. This threshold reflects an approximated of the minimum energy required to reconstruct a particle. As will become clear from Chapters 7 and 8 , the threshold is very conservative and does not impact the physics phase space.

2. The simulated interaction vertex of the neutrino has to be inside a Fiducial Volume (FV) defined in Figure 6.1. The size of the borders is $10 \mathrm{~cm}$ at every side of the TPC, except of the downstream end, where a $50 \mathrm{~cm}$ border is taken to allow the final state lepton to propagate far enough to be reconstructed.

Depending on the flavour of the interaction, the signal events will be referred to as $v_{e} \mathrm{CC}$ or $v_{\mu}$ CC. In this and the following chapters, passing rates for each step will be discussed for the Beam Off background sample and two here defined signals.

Normalisation In order to ensure that the simulated events correspond to the correct amount of collected data, a normalisation prescription is needed. This is performed by using the 


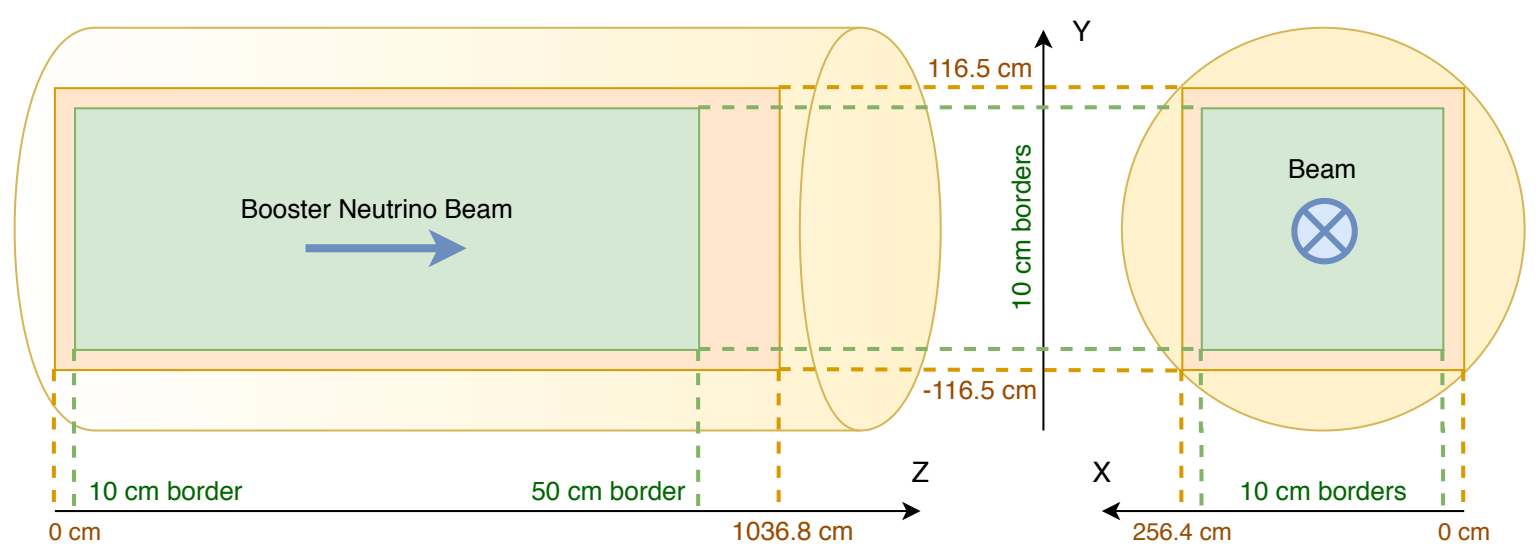

Figure 6.1: Fiducial Volume (FV) used in this thesis. The yellow cylinder represents the cryostat, the orange structure is the TPC and the green area is the FV. Both the $Y Z$ (left) and $Y Z$ (right) projections are shown.

number of collected Protons on Target (POT) and the corresponding number of event triggers. Event triggers are the number of beam spills received from the accelerator division signals as described in Section 4.1. The Beam Off sample is scaled to the Beam Off by scaling the amount of external triggers. The MC samples are normalised to match the POT exposure of the Beam On data by dividing each MC event by the simulated number of POT, and multiplying by the number of Beam On data POT.

To compare data and Monte Carlo, the following samples are used:

1. Beam On (data). Data events acquired from the BNB. In this chapter, a subset of the Run2 data-taking period is used (Oct 2016 - Jun 2017) corresponding to an exposure of $1.6 \times 10^{20}$ POT.

2. Beam Off (data). A sample corresponding to the same period is used. This sample serves to model the cosmic-only background events.

3. BNB $v(\mathbf{M C})$. Simulated sample in which every event contains a neutrino interaction inside of the cryostat. The energy and flavour of the simulated neutrinos follows the predictions from the Booster Neutrino Beam as described in Section 3.1.1. The cosmic activity is included using data Overlay.

4. BNB intrinsic $v_{e}(\mathrm{MC})$. Enriched electron neutrino sample, containing charged-current 
$v_{e}$ interactions inside the TPC. This sample can be used as a subset of the full simulated neutrino sample, providing enhanced statistics in the $v_{e} \mathrm{CC}$ signal category.

5. Neutrino dirt interactions (MC). Neutrinos can also interact outside of the cyostat, mainly in the $\mathcal{O}(10 \mathrm{~m})$ in front of the cryostat or in the cryostat vessel. The interaction products might enter the TPC and trigger the light system, possible causing backgrounds.

All the simulated samples use the Overlay method to include cosmic activity, meaning they are constructed by adding simulated neutrino information onto cosmic data background events as described in Section 4.2.4. Since all these samples correspond to different Beam On exposures, a normalisation factor has to be applied with the reference being the Beam On data-set. A more detailed discussion of normalisation can be found in [86]. 


\subsection{Optical selection}

The first step in identifying a neutrino interaction is to remove the cosmic background. This process starts from the Pandora reconstructed products - described in Section 4.2.3 and is illustrated in Figure 6.2. After the Pandora pattern-recognition algorithm suite has isolated individual interactions into reconstructed slices and removed the obvious cosmic activity, the remaining task is to identify which - if any - slice is associated to a neutrino interaction. Scintillation light information is used to reject slices incompatible with light recorded in-time with the beam. Additionally, topological cuts aimed at rejecting stoppingmuon events which enter the TPC are used. The NeutrinoID is at the core of all neutrino selections performed in this analysis; this first, common step is responsible for the majority of cosmic-rejection.

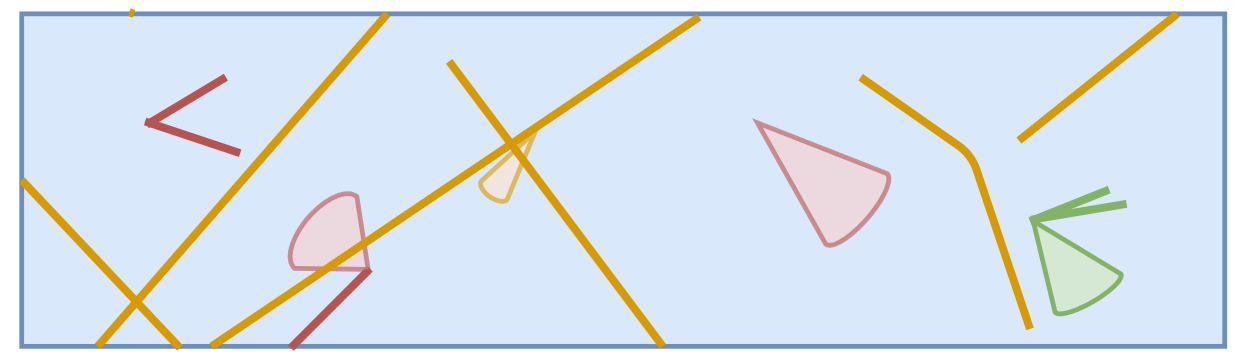

(a) Typical event with multiple interactions isolated by Pandora in cosmic muons (yellow) and neutrino-like slices (red and green).

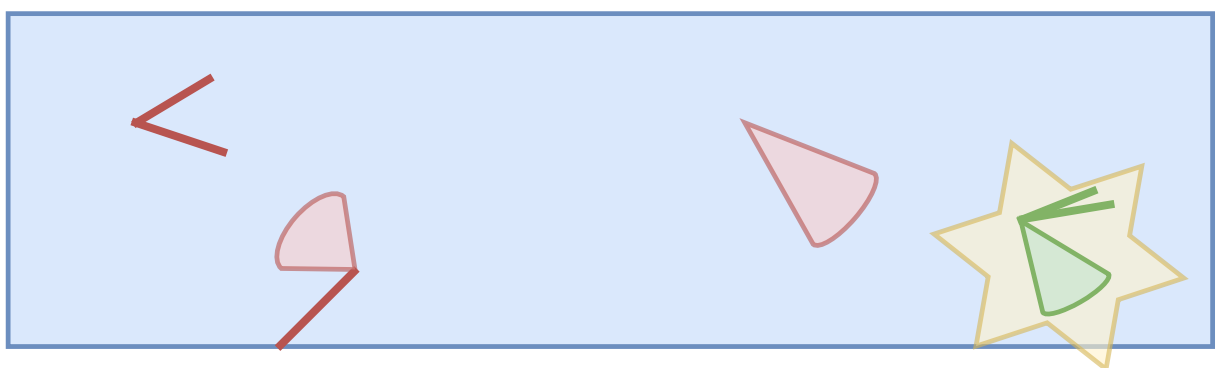

(b) Event after the removal of obvious cosmics tagged geometrically by Pandora. The neutrino slice (green) is selected using the light information.

Figure 6.2: Succession of steps in cosmic removal performed using Pandora's topological pattern recognition combined with scintillation light information. The lines and the cones represent track-like and electromagnetic shower particles respectively. 
To select the neutrino slice - or reject the event - the following procedure is followed; each step will be discussed in details in the next paragraphs:

1. Require a beam-triggered flash in the beam window.

2. Only consider slices that are compatible with the location and intensity of the flash.

3. Remove slices that contain Anode or Cathode Piercing Tracks (ACPT).

4. Remove slices that contain a stopping cosmic muon.

5. Leverage two addition handles to select the neutrino candidate out of the remaining slices and further reject events with a cosmic triggered flash.

- A topological SVM response, which categorises slices as cosmic- or neutrino-like.

- A flash-matching likelihood, which assesses how well the flash-hypothesis for the slice matches the beam flash.

\subsubsection{Flash selection}

Beam flash window To select the neutrino slice, we first require a flash in the beam window. To account for errors on the exact spill duration, start and end point of the spill window in data, an additional $40 \mathrm{~ns}$ is added to both sides, corresponding to an increase of $5 \%$ in the spill window duration. Therefore the window matched with the BNB spill is $3.16 \mu$ s to $4.84 \mu$ s with respect to the trigger time.

Common optical pre-filter The distribution of the reconstructed flash times is given in Figure 6.3. The common optical filter consists of applying a flash veto in the window between $1 \mu$ s and $3 \mu$ s and after $5 \mu$ s. In order to further reduce the data volume before applying TPC reconstruction algorithms, there is an additional offline filtering stage. It requires a minimum of $20 \mathrm{PE}$ fast scintillation light in a more narrow fraction of the $23.4 \mu \mathrm{s}$ beam discriminator window. This data-reduction is not applied to the simulation at this stage and explains the difference in the Beam On - Beam Off panel of Figure 6.3. 

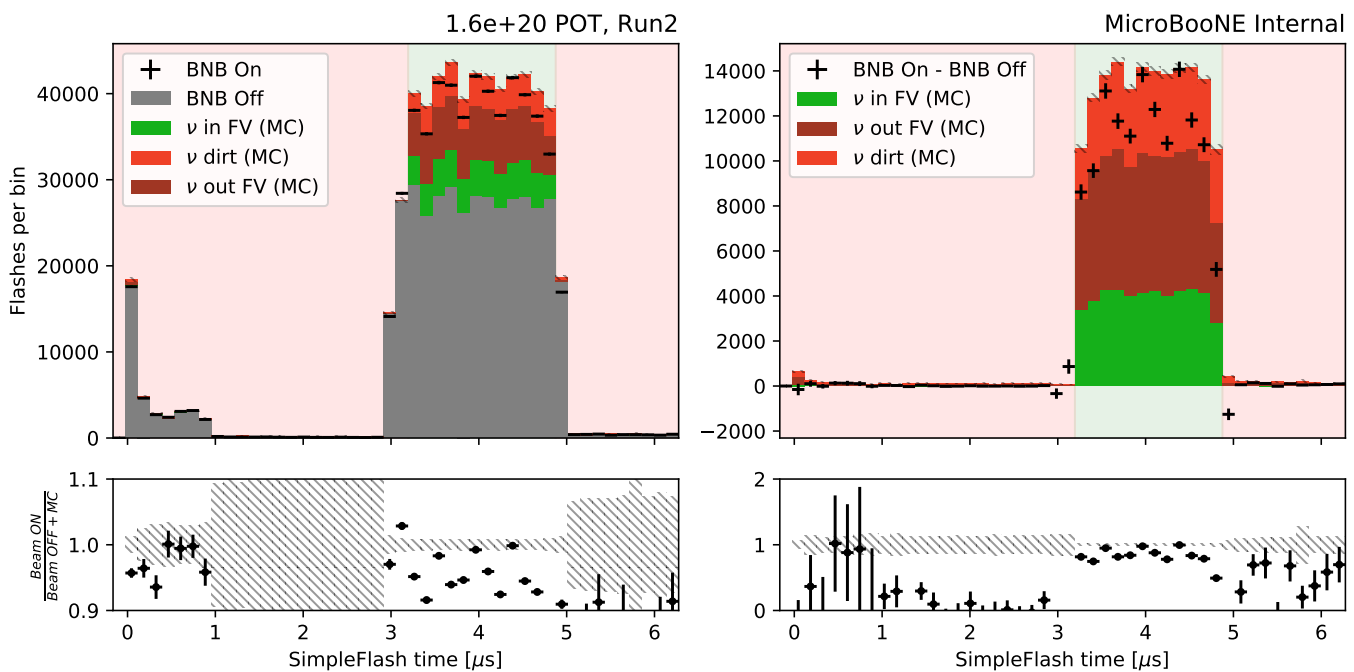

Figure 6.3: Flash times with respect to the beam trigger. The right panel is identical to the left one after the subtraction of the Beam Off data to allow for a direct simulation versus data comparison. The differences in normalisation are attributed to the additional offline beam quality cuts applied on Beam On data.

Events are selected if there is a flash with at least $50 \mathrm{PE}$ in the $1.68 \mu \mathrm{s}$ window corresponding to the BNB spill. This requirement leads to a selection rate of $(98.38 \pm 0.08) \%$ for the $v_{e}$ CC signal definition and $(98.12 \pm 0.13) \%$ for $v_{\mu} \mathrm{CC}^{\prime}$ s, as summarised in the first row of Table 6.1 at the end of this chapter.

\subsubsection{Flash compatibility with neutrino candidates}

In this section, strategies will be discussed to decide if an individual slice can be related to the beam flash, hence being a neutrino candidate. Furthermore, flashes outside of the beam window will be used to tag cosmic muon slices and tools to reject stopping muons slices are also included. The amount of slices per event ranges from $\approx 3.5$ in data event to approximately 4.5 in simulated signal events (see Figure 6.13). At the end of the NeutrinoID, there can be maximum one slice per event.

We introduce here two definitions which will be used further on to quantify the reconstruction quality of neutrino candidate slices in simulated events:

- purity: Consider all the reconstructed and clustered hits in the slice. The fraction of 


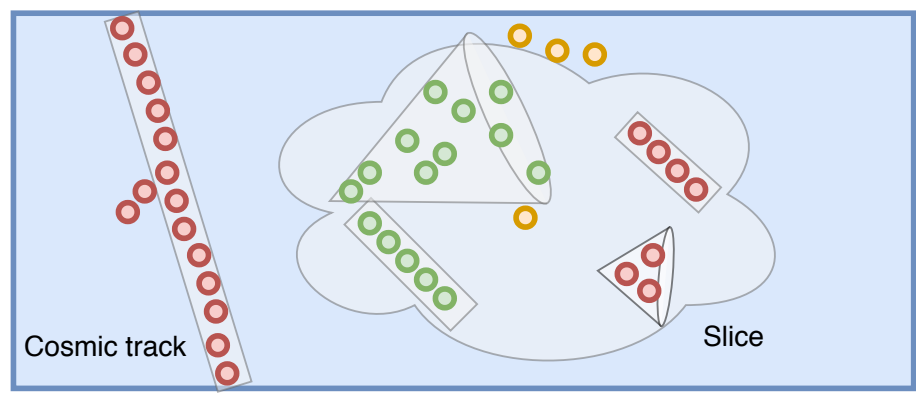

Figure 6.4: Schematic defining the slice purity and completeness. Hits are clustered into showers and tracks, indicated by cones and boxes respectively. The hits related to cosmic activity are coloured in red. Hits that originate from neutrino activity are shown in green (clustered and in the slice) or in orange (not clustered). The purity of the slice is defined as the number of green hits divided by the green plus red hits inside the slice. The completeness of the slice is defined as the green hits inside the slice divided by the green plus orange hits inside the whole event.

these hits truly induced by the neutrino simulated charge deposits is defined as the slice purity.

- completeness: Consider all reconstructed neutrino induced hits in the event, the fraction of these hits which are clustered into a certain slice is the neutrino completeness of the slice.

These definitions are clarified in Figure 6.4.

\section{Flash location}

In Section 6.2.1, the flash time and total number of Photo-Electrons were used. Instead of only looking at the total PE, one can also use the location of the flash. The location of flash centre - and the corresponding flash width - in the $Y Z$-plane are constructed by looking at the location and PE-count of the individual PMTs, see Figure 4.2. The variables of interest are obtained by taking the PE-weighted sum of the PMT locations and referred to as:

$$
\text { Flash } z \pm \text { Flashwidth } z \text { and Flash } y \pm \text { Flashwidth } y
$$

Additionally, using the 3D SpacePoints that make up each slice, the centre of deposited charge can also be defined as an average of the SpacePoints location, weighted by the collec- 
tion plane charge:

\section{Charge $x$, Charge $y$ and Charge $z$}

It is expected that this charge centre should be near the centre of the reconstructed flash if that neutrino candidate slice was the interaction causing the flash. The following four variables are constructed to compare the location in the $Y Z$-projection:

- Charge $z$ - Flash $z$ and (Charge $z$-Flash $z$ )/Flashwidth $z$

- Charge $y$ - Flash $y$ and (Charge $y$ - Flash $y)$ /Flashwidth $y$

Figures 6.5 and 6.6 illustrate the selection power and data/MC agreement for each of those variables. it should be noted that the plots are filled with neutrino candidate slices, typically multiple per event. The green shaded area is the selected part of the parameter space, candidates with values in the red shaded areas are discarded.
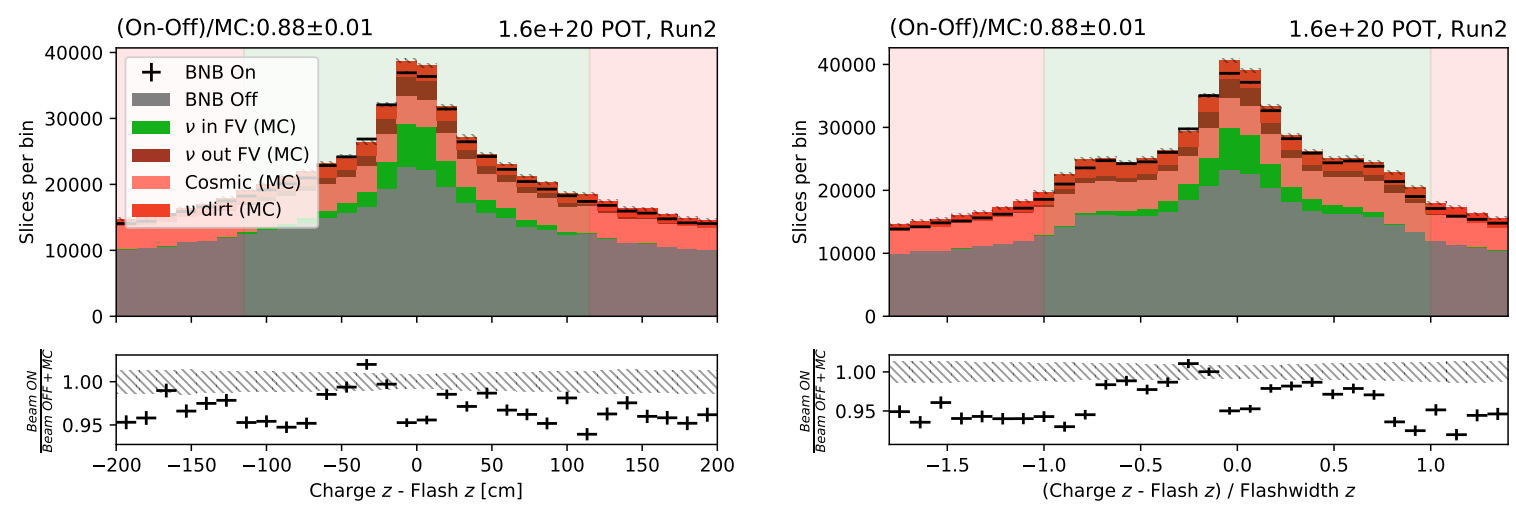

Figure 6.5: Requirement that the position of the beam flash and the centre of the charge distribution of the considered slice are in each others vicinity. Variables in the $z$ direction. The shaded green area indicated the accepted region. Slices in the red area are rejected.

In Figures 6.5 and 6.6, the histogram is filled for each slice. The BNB $v$ sample is split up in three parts:

- Cosmic $(M C)^{1}$ : slices with a neutrino completeness or purity below $50 \%$. These are considered to be badly reconstructed and we do not consider them valuable for the neutrino analysis.

\footnotetext{
${ }^{1}$ This category differs from the Beam Off events since the Cosmic $(M C)$ events contain a simulated neutrino interaction.
} 

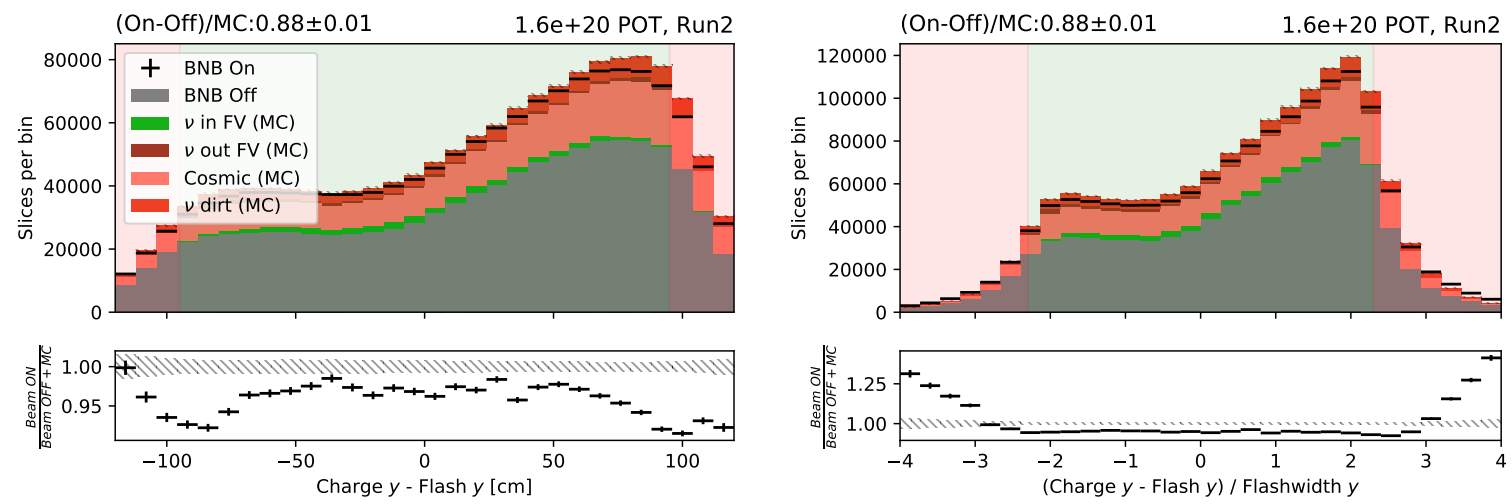

Figure 6.6: Requirement that the position of the beam flash and the centre of the charge distribution of the considered slice are in each others vicinity. Variables in the $y$ direction. The shaded green area indicated the accepted region. Slices in the red area are rejected.

- The remaining slices with a purity and completeness above $50 \%$ are split in:

$-v$ in FV (MC): the true neutrino vertex lies inside the fiducial volume as defined in Figure 6.1.

$-v$ out FV (MC): slices with the vertex outside the fiducial volume. These events are considered as a background if selected.

By definition of the completeness requirement, each event can only have one slice in one of the above defined categories.

The cuts are designed such that the fraction of $v$ in FV (MC) rejected by each individual cut is below $2 \%$.

\section{The relation between flash intensity, deposited charge and $x$-coordinate}

The next step is to relate the intensity of the flash with the amount of deposited charge. The relation between ionisation charge and scintillation light is partly dependent on the particle type through the dependence of recombination on the local ionisation density (see Section 3.2.2). This dependence is only of the order of $10 \%$, which will lead to a smearing in the Charge/Light ratio.

As was discussed in the context of cosmic muon in Figure 5.14, the flash intensity is strongly 
dependent on the $x$-position. The $x$-coordinate of the reconstructed SpacePoints is defined by the beam spill under the neutrino hypothesis. During the $1.6 \mu$ seam window, the ionisation electrons travel $\mathcal{O}(2 \mathrm{~mm})$, which is comparable to the inter-wire distance.

Therefore, it is natural to expect a relation between the $x$ location and the Charge/Light ratio. Note that light refers to the flash intensity in PE and charge refers to the deposited collection plane charge inside a slice, quoted in ADC. This relation is explored in Figure 6.7.
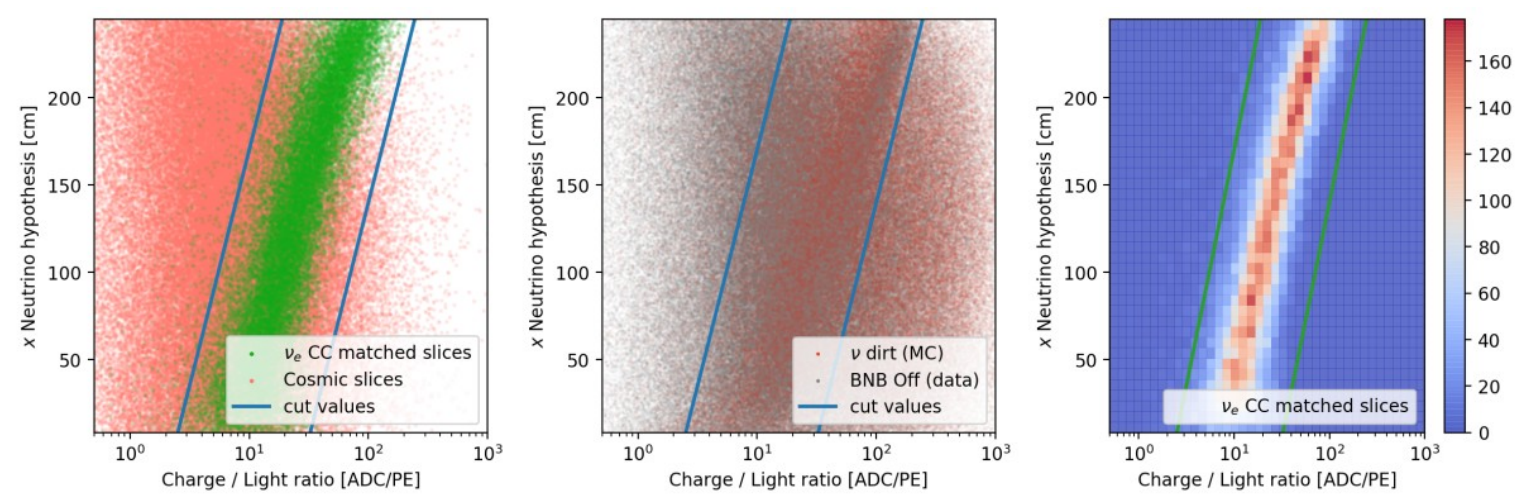

Figure 6.7: 2D scatter and density plots connecting the $x$-position and the collection charge of the slice with the flash intensity. The signal definition used in the left and right plots are $v_{e} \mathrm{CC}$, equivalent results are obtained for the $v_{\mu} \mathrm{CC}$ signal. The left and middle panels shows the separation between slices which are well reconstructed (high purity/completeness) and slices which are mostly cosmic background. The slices with values in the region defined by the two blue lines are accepted. The middle panel looks at the same variables, but now for Beam Off data and simulated dirt background events. The panel on the right contains the same events as the green scattered dots on the left but in a $2 D$ density plot to have a quantitative estimate of the signal rejection, which is below $2 \%$ for both signal definitions. The right and middle panels clearly demonstrate the rejection power in different background event categories.

A good separation power between neutrino slices and background slices is obtained with the following relation:

$$
\text { Charge } x=m \cdot \log \left(\frac{\text { collection plane charge [ADC] }}{\text { flash intensity [PE] }}\right)-q \text {, }
$$

where $m=270$ and $q \in[100,400]$. After defining the appropriate variable, the proposed cut becomes a rectangular cut in $q$. The data/MC comparison is given in Figure 6.8. 


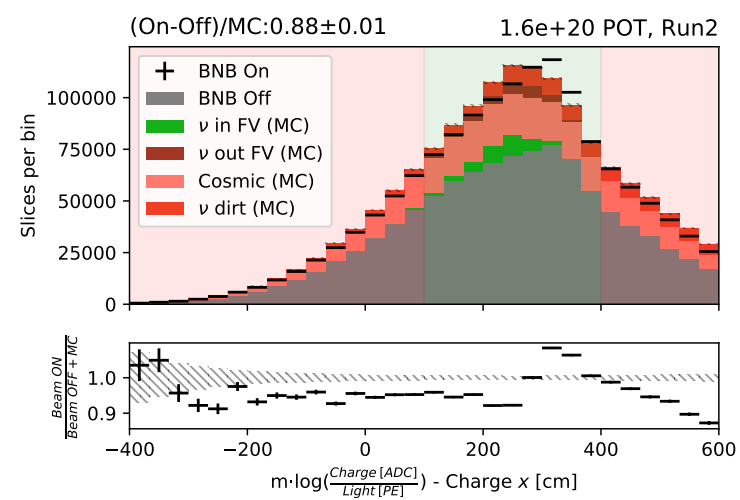

Figure 6.8: Rectangular cut combining the flash intensity with the collected charge and $x$-position for the slice. The variable on the horizontal axis is deduced from Equation (6.1). The shaded green area indicated the accepted region. Slices in the red area are rejected.

In all of the five rectangular cuts - indicated by the green shaded areas in Figures 6.5, 6.6 and 6.8 - data/MC disagreement can be seen. This is mitigated by relaxing the cuts and avoiding regions in which the disagreement is large. The basis of this disagreement is the MicroBooNE light simulation. A photon visibility library is constructed to reduce the computational load related to simulating photon trajectories with GEANT. The library predicts the acceptance for each of the 32 PMTs originating from a certain amount of isotropic emitted scintillation light at a point $(x, y, z)$ inside the cryostat. To mitigate the risk of the data/MC disagreement, the systematic uncertainty is estimated by the production of samples with a different light yield and/or light modelling. This will be discussed further in Section 9.1.3.

\section{Anode-Cathode Piercing Track veto}

If a track crosses the entire TPC by entering and exiting though one of the four sides except the cathode/anode, it is geometrically identified as a crossing muon. If such a track enters or exits through the cathode or anode, its $x$-coordinate will be shifted to match the beam window. This is illustrated in the left panel of Figure 6.9 and such tracks are referred to as Anode or Cathode Piercing Tracks (ACPT). We now use the cosmic discriminator flashes to identify such a background. In the situation illustrated, the track crosses the anode plane in $x_{A}$ at $t_{0}$ and would therefore have created a cosmic discriminator flash at the same time. 


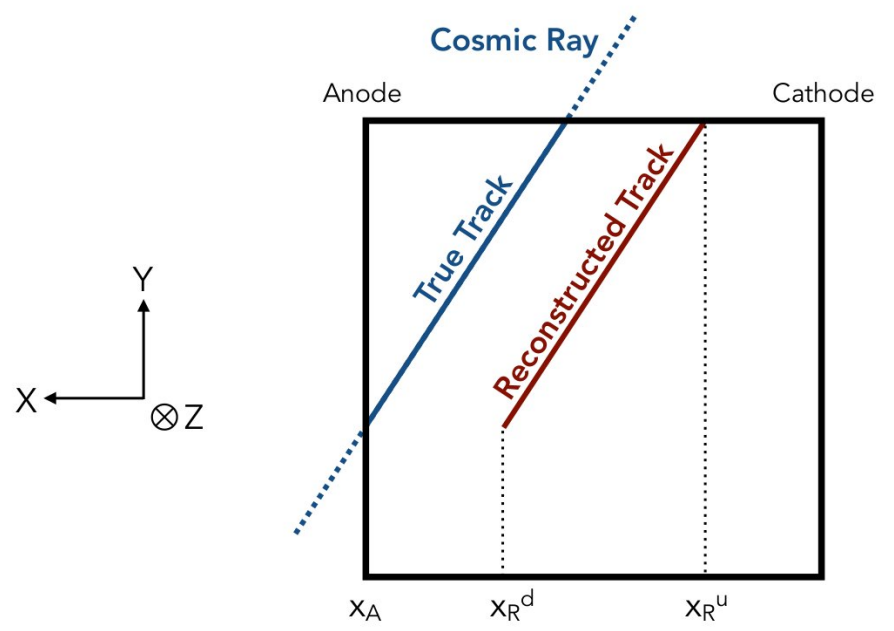

(a)

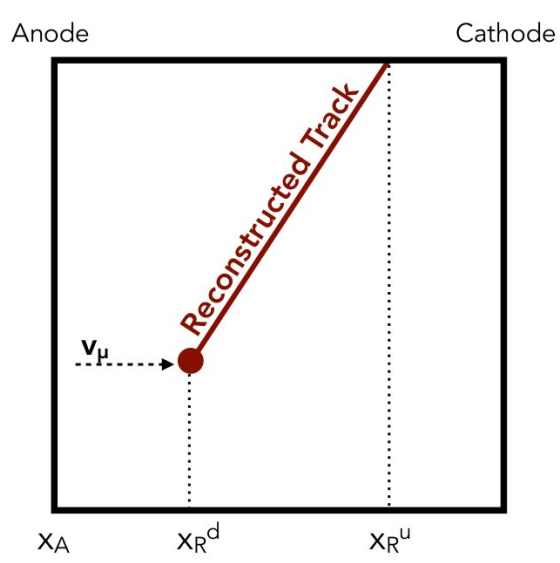

(b)

Figure 6.9: Reconstructed tracks need to be corrected for their time offset introduced by the finite velocity of the drifting electrons. In (a), a true cosmic muon track enters the detector from the top and exits from the anode plane. Reconstructed under the neutrino hypothesis, the $x$-position gets shifted to match the beam window. In (b), a neutrino interacts in the detector producing a muon that exits from the top. The signature is similar to the cosmic ray in (a). Figure from [58].

The track got reconstructed at $x_{R}^{d}$ under the beam flash hypothesis at time $t_{v}$. Therefore:

$$
t_{0}=t_{v}-\frac{x_{A}-x_{R}^{d}}{v_{d r i f t}}
$$

where $v_{\text {drift }}$ is the electron drift velocity of $114 \mathrm{~cm} / \mathrm{ms}$ in MicroBooNE. Of course, for a track pointing towards the cathode instead of the anode, one looks at a hypothetical point $x_{C}$ where the cosmic muon would intersect the cathode at $t_{0}$.

The next step is to look for a flash at the time $t_{0}$. The difference between $t_{0}$ and the closest flash is given in Figure 6.10. If a cosmic flash is found within $10 \mu$ s of the hypothetical anode/cathode crossing time, the slice is assumed to contain an ACPT and is tagged and removed as a possible neutrino candidate. To reduce the chance of random coincident cosmic flashes, the track and the flash are required to be in each others vicinity in the $Y Z$-plane. 

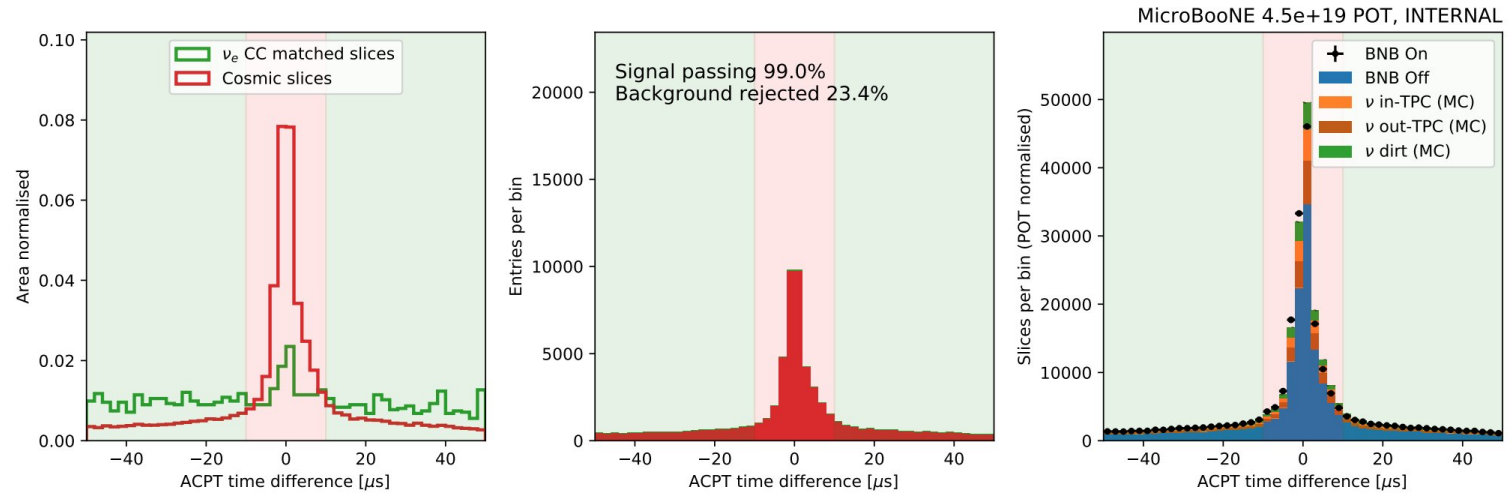

Figure 6.10: Rectangular cut aimed at removing slices containing Anode or Cathode Piercing Tracks. The variable on the horizontal axis indicates the time difference between the track crossing the anode/cathode plane and the nearest cosmic discriminator flash, as defined in Equation (6.2). The events in the shaded red region are discarded. The flat distribution of signal slices in the red region corresponds to random time coincidences and is below $1 \%$. The left panels contains area normalised histograms to distinguish the signal and background shapes. The right panels shows the data/MC agreement.

\section{Stopping muon tagger}

A residual background of cosmic activity not targeted yet is stopping muons. As was shown in Figure 5.4, approximately $12 \%$ of the muons entering the TPC stop inside.

A set of distinct algorithms are in place to reject this type of background and are described in detail in [58]. Two strategies are used to identify the direction of the muon track. First, the $\mathrm{d} E / \mathrm{d} x$ profile over the length of the track can be used to identify a Bragg peak. If a Bragg peak is found on the start/end of the track that is located inside the FV, then the track is considered a stopping muon and the slice is rejected. The second method relies on Multiple Coulomb Scattering (MCS).

A charged particle traversing LAr is deflected by many small-angle interactions. The dominant scattering process is Coulomb scattering from nuclei. The Coulomb scattering distribution is roughly Gaussian, and - for small angles - the width is given by:

$$
\theta_{0} \approx \frac{13.6 \mathrm{MeV}}{\beta c p} z \sqrt{x / X_{0}}\left(1+0.038 \ln \left(x / X_{0}\right)\right)
$$


Here, $p, \beta c, z$ are the momentum, velocity, and charge number of the incident particles. In this thesis, MCS will only be used for muons and therefore $z=1$. The ratio $x / X_{0}$ is the thickness of the scattering material in radiation length. The scattering process is illustrated in Figure 6.11. A general description can be found in the PDG [12] and the specific parametrisation and application in the MicroBooNE experiment can be found in [87].

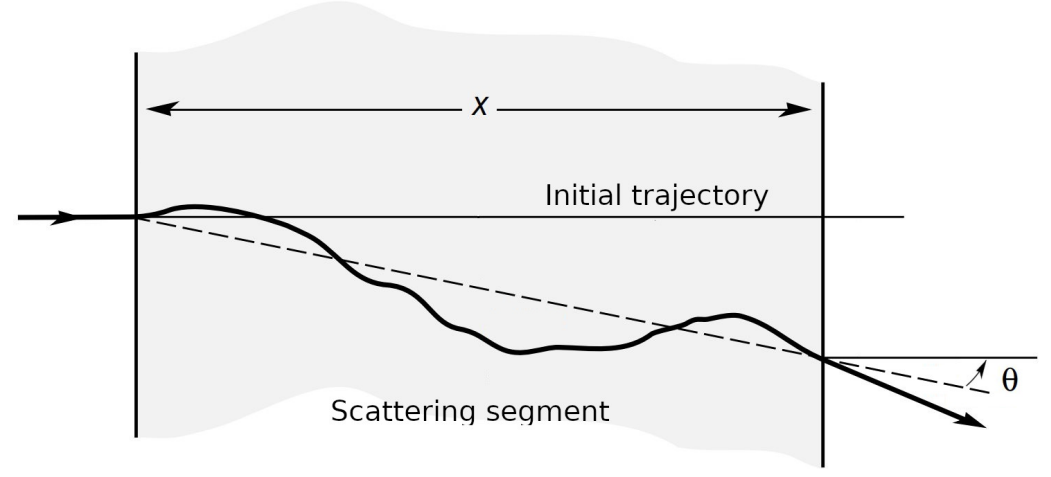

Figure 6.11: Quantities used to describe multiple Coulomb scattering. The reconstructed track is divided in segments with a length $x$ of $\mathcal{O}(10 \mathrm{~cm})$. For every segment, the scattering angle is calculated. Figure adapted from [12].

The power of Equation (6.3) stems from its dependency on the muon momentum. In Chapter 7, MCS will be used to estimate the momentum of muon tracks, especially in a selection looking for uncontained muons. Furthermore, since muons deposit energy while traversing the LArTPC, their momentum decreases and their scattering angle increases. This characteristic is exploited to identify the muon direction and therefore to remove stopping cosmicmuon slices.

Assuming the track is a muon track and its highest y-coordinate is outside the FV, the track scattering is fit using the MCS formula in both directions. The fit uses a log-likelihood approach and is obtained by fitting the MCS hypothesis to the track in the down going and up going direction, respectively called $\log \mathcal{L}_{\text {down }}$ and $\log \mathcal{L}_{\text {up }}$. The difference between these two is considered:

$$
\Delta \log \mathcal{L}_{\mathcal{M C S}}=\log \mathcal{L}_{\text {down }}-\log \mathcal{L}_{\text {up }}
$$

If the track belongs to a cosmic-ray muon $\Delta \log \mathcal{L}_{M C S}$ is expected to be positive. 


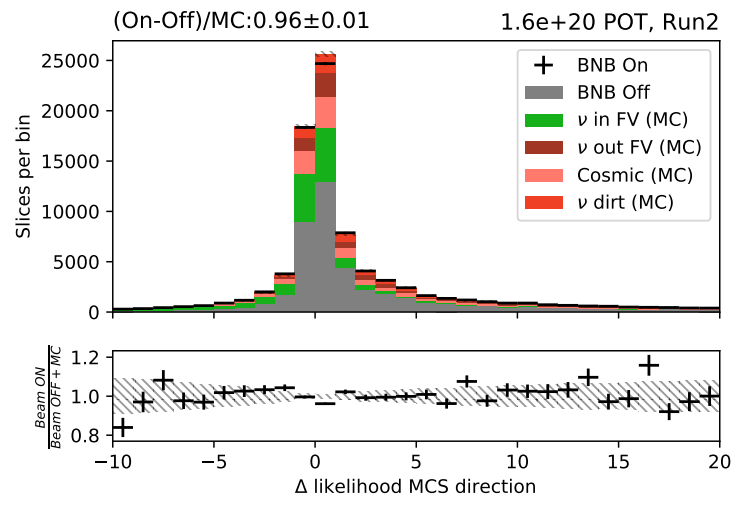

Figure 6.12: Multiple Coulomb Scattering likelihood to identify stopping muon background. The variable on the horizontal axis is defined in Equation (6.4) and motivated in the text.

Figure 6.12 shows this variable for slices passing the previously described cuts. No hard rectangular cut is made on any of the stopping muon variables, instead a combination of these variables is made to reject slices that are likely to contain a stopping muon.

\section{Number of slices compatible with the flash}

Figure 6.13 shows the cumulative effect of the cuts introduced in this section on the number of neutrino candidates (slices) per event. It can be appreciated that, while in the beginning, every event has at least one neutrino candidate, at this point, the majority of events do not have any compatible slice remaining (the 0 slices bin in the right panel). Events with no compatible slices after the described cuts are rejected from neutrino searches. The flash compatibility requirements, combined with the beam flash requirement itself, lead to a selection rate of $(84.17 \pm 0.22) \%$ for the $v_{e}$ CC signal definition and $(84.12 \pm 0.35) \%$ for $v_{\mu}$ CC's, which is summarised in the second row of Table 6.1. The loss in efficiency of signal events can be explained by:

- The neutrino interaction was not reconstructed by Pandora.

- There was no reconstructed cluster on the collection plane. Notice that this selection step requires deposited charge on the collection plane to calculate the discriminating variables.

- There was a reconstructed neutrino candidate but its charge deposition was incom- 
patible with the flash. This can happen when the neutrino interaction is split into multiple Pandora candidates or when the candidate is contaminated by cosmogenic activity.

The event rejection power of this step on the Beam Off events is approximately a factor 4. From the right panel in Figure 6.13, it can be seen that the only bin with significant data/MC disagreement is the one in which no slices remain and the event gets rejected. The differences therefore are assumed to be caused by background events and are of limited importance for neutrino analyses.
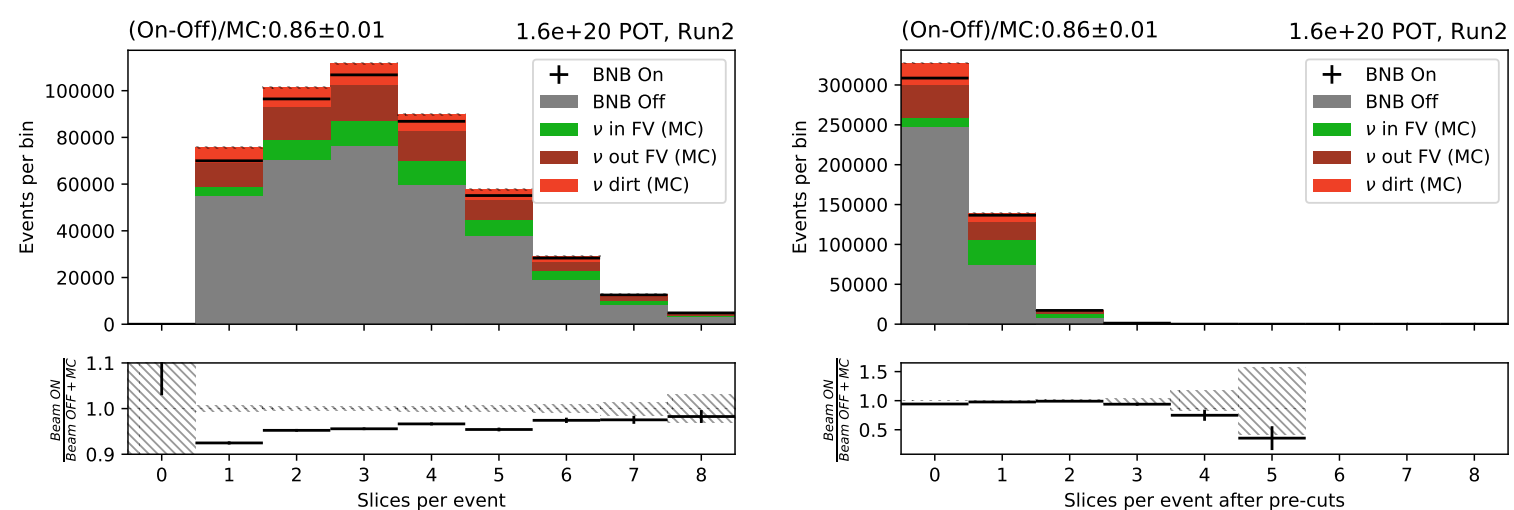

Figure 6.13: Number of neutrino candidates before (left) and after (right) the slice rejection steps. Events that have no remaining slices after this stage are discarded. 


\subsection{Neutrino candidate selection}

As demonstrated in Figure 6.13, it is unlikely that there is more than one slice passing the procedure outlined in the previous section. For the $v_{e}$ CC and $v_{\mu}$ CC signal sample, which are subsets of the green " $v$ in FV" category in the right panel of Figure 6.13:

- No remaining slices (16\%): the event is rejected.

- Exactly one remaining slice (73\%): The event is selected with the slice as neutrino candidate.

- More than one slice remaining (11\%): The preferred slice is chosen using the topological score and/or flash-matching

The topological score and the flash-matching will now be introduced.

\subsubsection{Topological Support Vector Machine}

The topological score is the response of a Support Vector Machine (SVM) aimed to classify slices in two categories: neutrino or cosmic. A SVM model is a representation of the examples as points in a high dimensional space, mapped so that the examples of the separate categories are divided by a clear gap that is as wide as possible. The variables used for the model are:

1. Variables calculated from the slice interpreted under the neutrino hypothesis:

- The number of final state particles.

- The number of reconstructed hits.

- The $y$ position of the neutrino vertex.

- $z$-component of the vector sum of the final state particle directions, weighted by the number of hits in each particle. This variable measures the alignment of the interaction with the Booster Neutrino Beam direction.

- The number of reconstructed 3D Spacepoints within $10 \mathrm{~cm}$ of the vertex. 
- The ratio of the eigenvalues of a Principal Components Analysis (PCA) including the $3 D$ Spacepoints within $10 \mathrm{~cm}$ of the vertex.

2. Variables calculated from the longest track in the slice, when interpreted under the cosmic hypothesis:

- The $y$-component of the track direction. Aimed to intercept downward going muon tracks.

- The angular difference between the direction of the start and the end of the track.

- The number of reconstructed hits in the track.

- The fraction of hits in the slice that are part of the track.

The response of the model is shown in Figure 6.14.

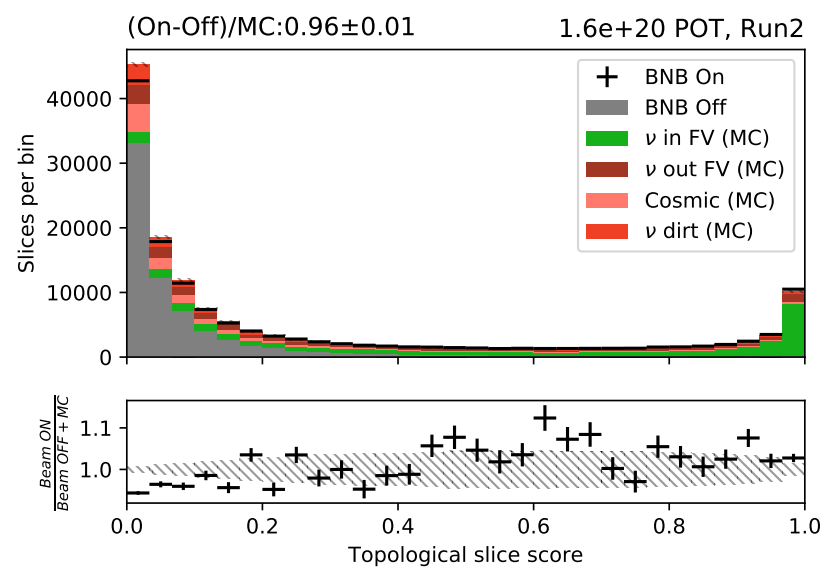

Figure 6.14: The response of the Pandora topological SVM. All cosmic background categories have a low response while the neutrino signal events (green) are piled up on the right at high score. No cut is applied directly on this variable.

The topological score is only used in the NeutrinoID if at least two slices pass the procedure described in Section 6.2. Of all original slices in the event, consider the one with the highest topological score. If this slice is among the remaining slices after the cuts previously described (Section 6.2), this slice is selected and the other slices in the event are rejected.

Of the $11 \%$ simulated signal events with multiple slices remaining, $9.5 \%$ is selected by this method. If the slice with the highest topological score did not pass the cuts described 
previously, the topological score and the optical information are in disagreement and flashmatching is used to make the final slice decision.

\subsubsection{Flash-matching}

Flash-matching is the process in which an estimated flash object - a flash hypothesis - is created for a cluster of deposited charge in the TPC. More specifically, in this section, flashmatching will be used to construct a flash for every neutrino slice that passed the flash compatibility cuts. These flash hypotheses are then compared with the reconstructed beam flash.

The creation of a flash hypothesis is illustrated in Figure 6.15:

1. For every slice, the spacial distribution of the deposited charge on the collection plane wires is used as input.

2. The collected charge is converted to an estimation of the emitted scintillation light for every SpacePoint. To attain this goal, the charge [ADC] is first translated in deposited energy $[\mathrm{MeV}]$ and thereafter into scintillation light [number of photons]. The last step is particle-type dependent and the charge-to-light conversion therefore differs between tracks and showers according to the light yield in liquid argon.

3. The scintillation light is propagated to the PMTs. A library is constructed, containing the solid angle covered by each PMT w.r.t. the location of the SpacePoint. Furthermore, a global quantum efficiency coefficient is taken into account, representing the scintillation light conversion by the TPB and the PMT gain. Variations during the MicroBooNE operation are taken into account through automatic gain calibration [63].

This PE hypothesis for each PMT is constructed and compared to the reconstructed flash. Given a single candidate slice, the algorithm calculates the following $\chi^{2}$-like metric between the observed and hypothetical spectra:

$$
\chi^{2}=\sum_{i=1}^{N=32} \frac{\left(O_{i}-H_{i}(\rho)\right)^{2}}{O_{i}+H_{i}(\rho)} .
$$




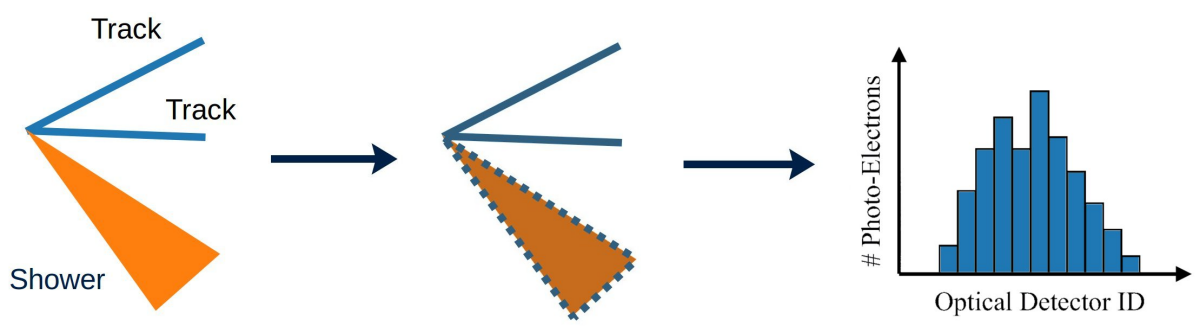

Figure 6.15: The different steps in the flash-matching process illustrated. The tracks and shower objects associated with a neutrino candidate slice are translated into a scintillation light estimate. The next step is the propagation of the light to create a flash hypothesis, consisting of a number of PE for each PMT.

Here, $H_{i}(\rho)$ is the PE hypothesis considering the charge distribution $\rho$ for a certain slice. $O_{i}$ is the PE measurement for PMT $i$. The distribution of the flash-match $\chi^{2}$ is given in Figure 6.16. It can be seen that the distribution in data is broadened compared to MC, therefore, the $\chi^{2}$ metric is only used relative between objects in the event, as described below and in the next section.

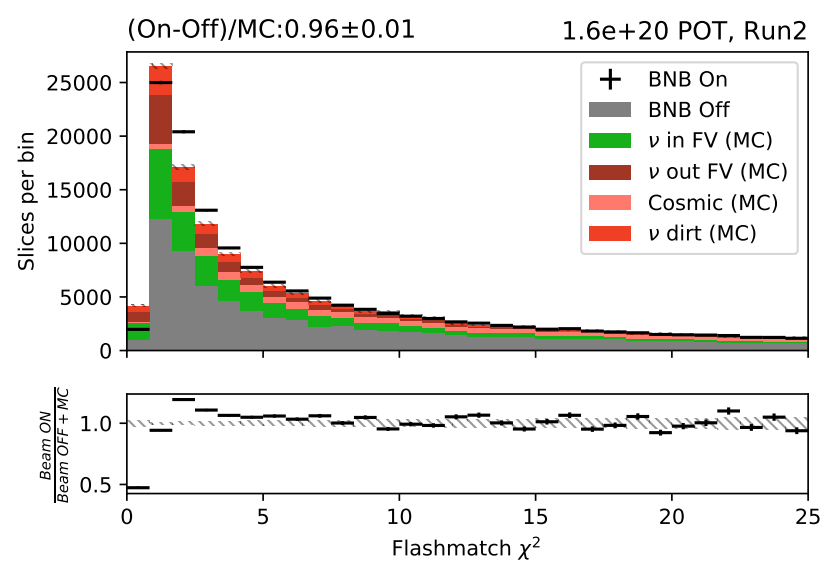

Figure 6.16: Flash-match $\chi^{2}$-like metric for all slices remaining after the selection described in Section 6.2.2.

In the events with multiple viable neutrino candidate slices, where the slice with the highest topological score was discarded by one of the rectangular cuts of Section 6.2 - this corresponds to approximately $1.5 \%$ of both $v_{e}$ CC and $v_{\mu}$ CC signal events - the $\chi^{2}$ flashmatching metric is used. The slice with the smallest value is kept for the further event selection, the other candidates are rejected. An example of how the hypothesis flash objects 
compare with the reconstructed flash is given in Figure 6.17. The optical flash registered by the PMT system is shown in shaded blue and compared with the flash hypothesis constructed from the charge corresponding to four different slices in the same event.

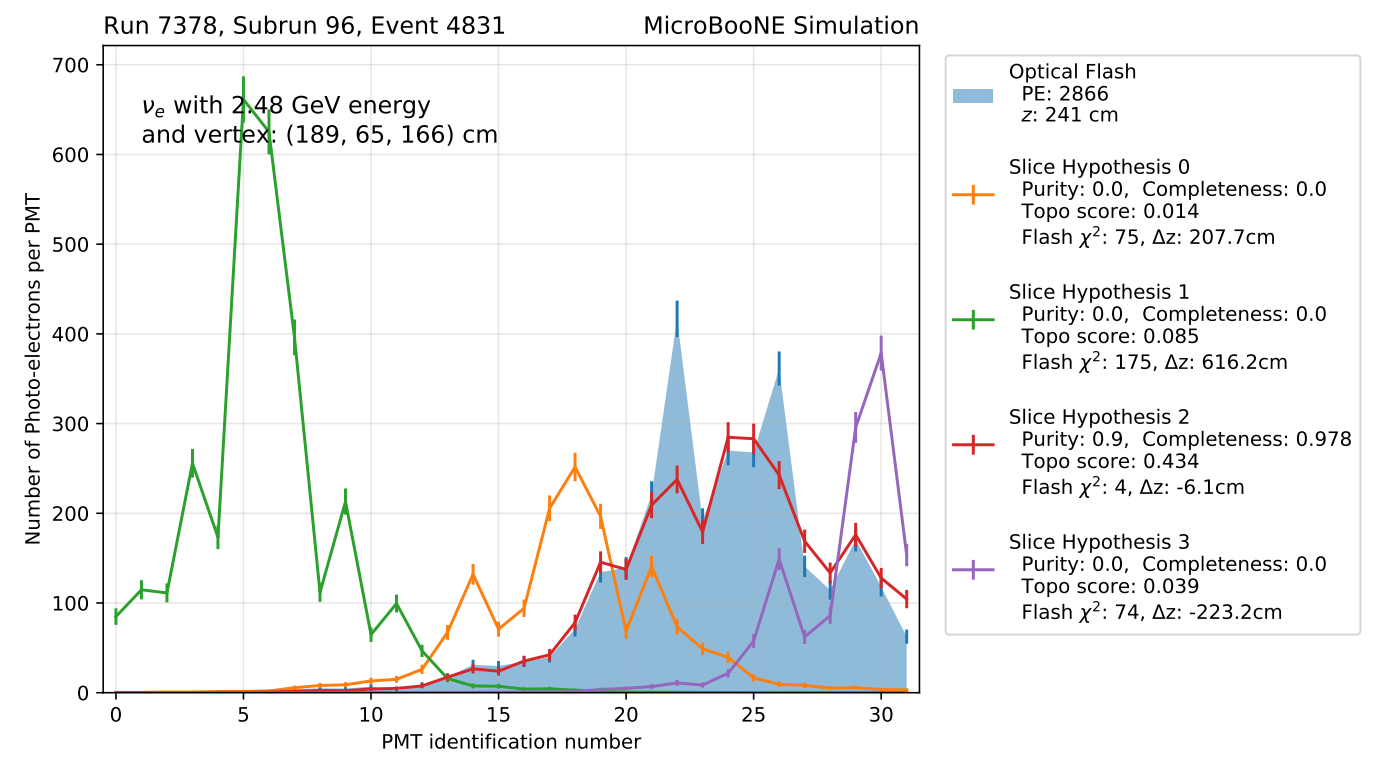

Figure 6.17: Flash-matching example for a simulated $v_{e} \mathrm{CC}$ event with a true neutrino energy of $2.5 \mathrm{GeV}$. The reconstructed optical flash (blue) is compared to the flash hypothesis of four neutrino candidates (coloured lines). $\Delta z$ is Charge $z$ - Flash $z$. Slice 2 (red) has the lowest $\chi^{2}$-value and is selected. Note that the selected slice also is the closest to the flash - smallest $|\Delta z|$ and has the highest topological score. The purity and completeness serve as a truth-level based indication of the reconstruction quality as introduced in Section 6.2.2. The horizontal axis shows the PMT number.

Complementarity of the topological score and the flash-match $\chi^{2} \quad$ Figure 6.18 contains three $2 D$ histograms, illustrating the correlations between the neutrino energy, the topological score and the flash-match $\chi^{2}$. It can be seen that for $v_{e}$ CC with $E\left(v_{e}\right) \lesssim 0.8 \mathrm{GeV}$ the topological score starts performing worse, resulting in a more uniform response. On the contrary, the flash-match $\chi^{2}$ tends to have lower values for lower energy events. This complementarity can be exploited in a $2 D$ cut based on both variables. No such cut was performed at this stage to leave the tailoring to a specific energy range or signal topology as much as possible to subsequent exclusive selections. 
$v_{e}$ CC perfect slices after optical pre-cuts
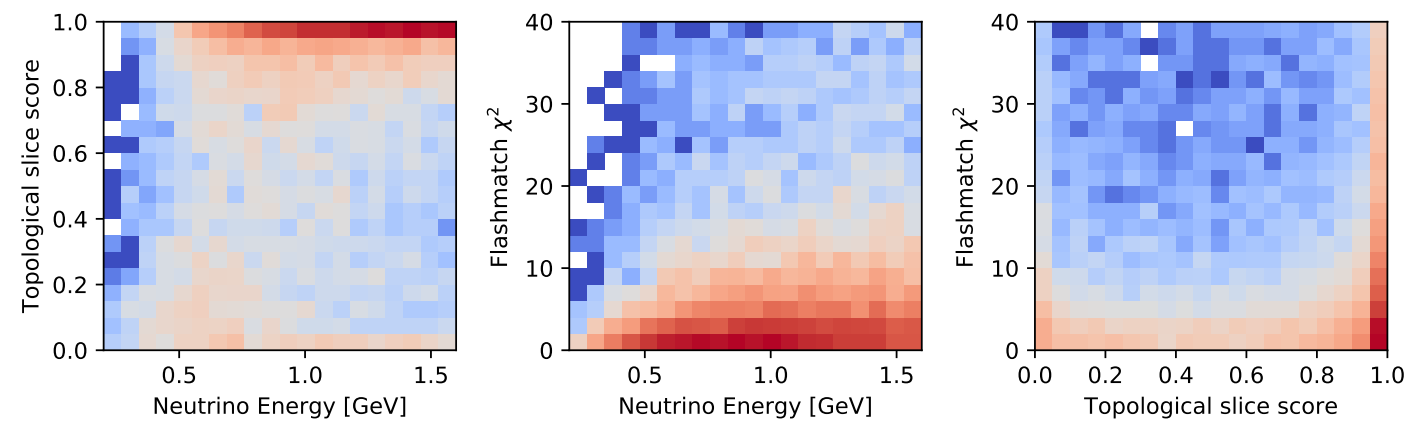

Figure 6.18: Relation between the topological score, the flash-match $\chi^{2}$ and the neutrino energy for $v_{e}$ CC reconstructed passing the NeutrinoID. Only well reconstructed slices with high completeness and purity are included in the histograms to disentangle correlations from reconstruction effects. The colour scale is logarithmic and represents the number of events per bin.

\subsubsection{Obvious cosmic flash-matching}

At this point in the NeutrinoID, each event has a maximum of one remaining slice, which will be referred to as the neutrino candidate. Most background events do not have any remaining slices and are discarded (see Table 6.1). One additional cut is applied to further reject events that were triggered by cosmic activity.

In most of the triggered events - approximately $6: 1$ - the beam flash will be caused by cosmogenic activity instead of neutrino activity. In that case, the beam flash should likely be matched with obvious cosmic muon activity (orange objects in Figure 6.2). Therefore, we now calculate the flash hypothesis and the corresponding flash-match $\chi^{2}$ for each of the obvious cosmics. Define now:

$$
\chi^{2} \text {-cosmic }=\min _{\{\text {obvious cosmics }\}}\left(\chi^{2}\right)
$$

As the flash-match $\chi^{2}$ corresponding to the obvious cosmic that matches the best with the beam flash. We can now compare this $\chi^{2}$ with the $\chi^{2}$ of the neutrino candidate found in Section 6.3.2. The ratio of the two is given in Figure 6.19. By rejecting events where an obvious cosmic matches the flash significantly better than the slice that was though to be the 
neutrino slice, the event is rejected. This additional selection step removes approximately $20 \%$ of the remaining Beam Off event, while removing $<1 \%$ of the $v_{e}$ CC and $v_{\mu}$ CC signals.
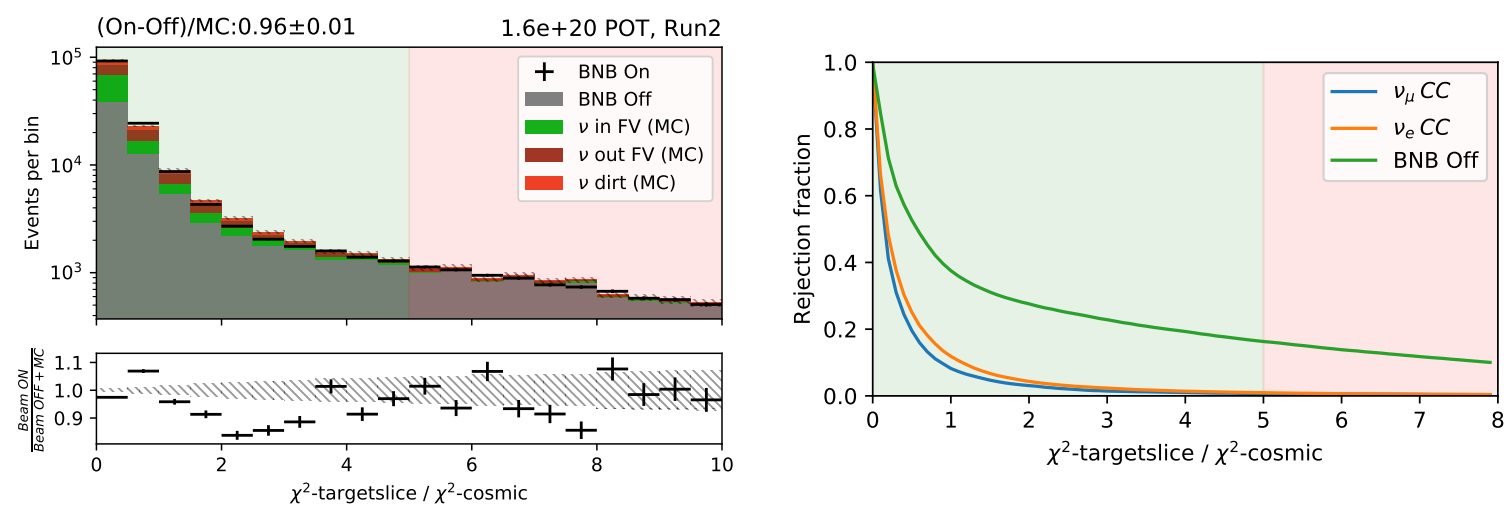

Figure 6.19: Beam Off rejection using the flash-matching ratio between the suggested neutrino slice and the most compatible obvious cosmic. (Left) Data/MC comparison of the ratio. (Right) the rejection power - on top of other methods described in this chapter - as a function of the cut value. It is clear that the signal categories are nearly unaffected. The events in the red shaded area are rejected. 


\subsection{NeutrinolD performance}

The passing rates for the different event samples of interest are given the last row of Table 6.1. The number of events of the Beam Off background category is reduced by a factor 7. The fraction of rejected slices in Beam Off off events is $95.2 \%$. A similar slice rejection efficiency of $92.8 \%$ is obtained in the simulated dirt event background. Overall the number of slices that need to be considered for further analysis is reduced by a factor 20 . The first two columns list the signal efficiency for $v_{e} \mathrm{CC}$ and $v_{\mu} \mathrm{CC}$, respectively $(83.37 \pm 0.23) \%$ and $(83.51 \pm 0.35) \%$.

Table 6.1: Summary of the NeutrinoID passing rates for different samples of interest and different stages in the selection. The statistical error are $\mathcal{O}(0.1 \%)$.

\begin{tabular}{lccccc}
\hline & $v_{e}$ CC & $v_{\mu}$ CC & Beam Off & Beam On & Dirt sample \\
\hline Beam flash & $98.4 \%$ & $98.1 \%$ & $69.8 \%$ & $75.8 \%$ & $73.1 \%$ \\
Flash compatibility & $84.2 \%$ & $84.1 \%$ & $17.5 \%$ & $25.4 \%$ & $23.0 \%$ \\
Selected by NeutrinoID & $83.4 \%$ & $83.5 \%$ & $14.7 \%$ & $23.1 \%$ & $22.8 \%$ \\
\hline
\end{tabular}
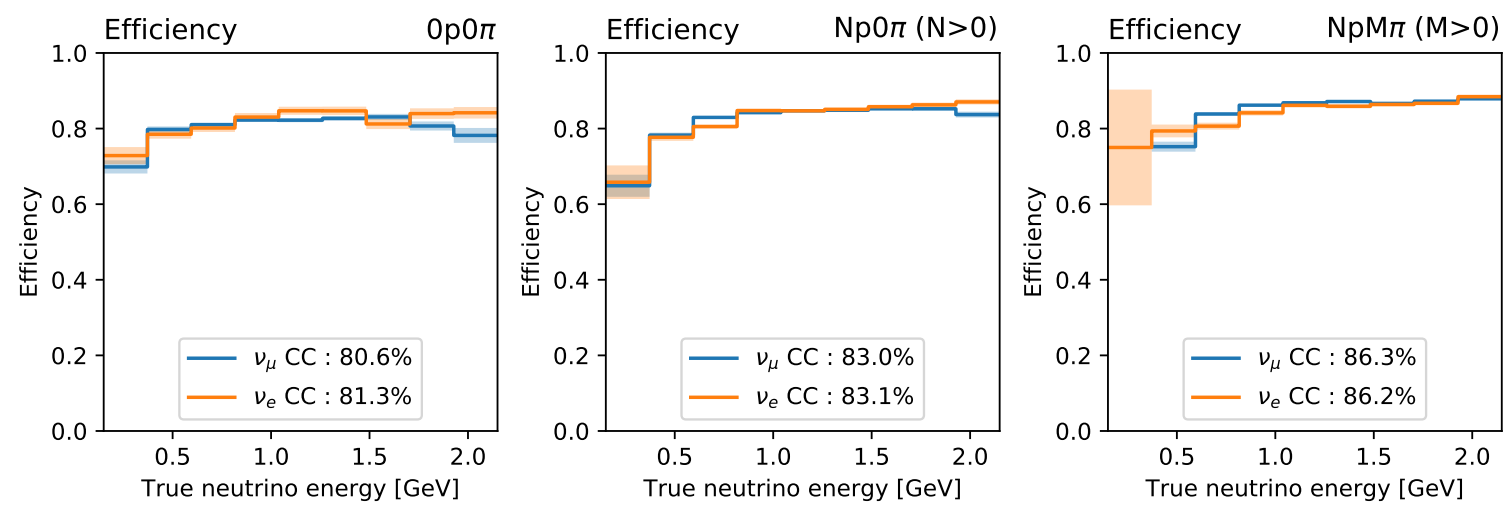

Figure 6.20: NeutrinoID efficiency as a function of neutrino energy for sub channels of $v_{\mu}$ CC and $v_{e}$ CC events. The range of the horizontal axis is $0.15 \mathrm{GeV}$ to $2.15 \mathrm{GeV}$. The shaded band corresponds to the statistical uncertainty.

Figure 6.20 shows the NeutrinoID efficiency as a function of neutrino energy for both $v_{\mu} \mathrm{CC}$ and $v_{e}$ CC signal events as introduced in Section 6.1. Subdivided in three channels:

- 0p: No protons or pions in the final state above $40 \mathrm{MeV}$ kinetic energy. The signature 
is a single muon track.

- $0 \pi \mathrm{N} p$ : At least one proton with $E_{k i n}(p)>40 \mathrm{MeV}$ in the final state but no pions above the kinetic energy threshold of $40 \mathrm{MeV}(\mathrm{N}>0)$.

- $\mathrm{M} \pi \mathrm{N} p$ : At least one pion with $E_{k i n}(\pi)>40 \mathrm{MeV}$, no restrictions on the number of protons $(\mathrm{M}>0)$.

The loss of approximately $17 \%$ of the signal events can be broken down in two main causes:

Pandora mis-reconstruction The neutrino candidate slice was not or was badly reconstructed. To attain the physics goals set by MicroBooNE and discussed in Section 3.3, detailed reconstruction of the vertex position, the tracks and the showers in the event is essential. Especially, to resolve the Low Energy Excess, the reconstructed neutrino energy resolution is important. Therefore, it is elucidating to look at the performance of the NeutrinoID for well reconstructed slices. Well-reconstructed is in this chapter defined as at least $80 \%$ neutrino hit purity and completeness.

Off the $\approx 16.5 \%$ of rejected signal events, it is found that $11.0 \%$ had no well reconstructed slice for $v_{e}$ CC events, and $10.0 \%$ in case of the $v_{\mu}$ CC events. Therefore, approximately $2 / 3$ of the inefficiency can be explained by reconstruction effects. Note that, for the ease of downstream analyser, it is actually positive that these events get discarded.

Flash reconstruction As will be further discussed using the right panel of Figure 6.22, at high $x$-location, the flash reconstruction efficiency drops. This is due to the low acceptance in combination with the low coverage of the PMT system for events near the cathode. The effective threshold of $\approx 30$ PE was lowered to experiment with dimmer flashes. Nonetheless, due to imperfections in the light simulation, the data/MC agreement deteriorates significantly when lower light signals are included. The inefficiency due to the flash creation is $\approx 2 \%$ for both signal definitions.

The flash reconstruction additionally leads to another inefficiency: as was described in Section 4.2.1, each reconstructed flash is integrated over a $8 \mu$ s interval. In Section 5.3 it was 
measured that the rate of cosmic interactions creating a flash is $\approx 4.5 \mathrm{kHz}$. Therefore, there is a $\mathcal{O}(4 \%)$ chance that the reconstructed flash object is contaminated with a light from cosmic activity. If this is the case, the compatibility of the flash object with the slice is no longer guaranteed and the event is often rejected.

Unresponsive wire region Approximately $10 \%$ of the wires in MicroBooNE are unresponsive (Appendix A). The main contribution to the NeutrinoID inefficiency is due to the zone between $z=675 \mathrm{~cm}$ and $775 \mathrm{~cm}$ on the collection plane, creating a blind spot. While in this region the induction planes can still be used to retrieve parts of the interaction, several cuts in this chapter rely heavily on both the amount and the reconstructed position of the collection plane charge. It is estimated that this is an $\mathcal{O}(7 \%)$ effect. Nonetheless, it was chosen to include the dead wire region in the fiducial volume (Figure 6.1) since there is still a non-zero efficiency and good purity, as is shown in the right panel of Figure 6.21.
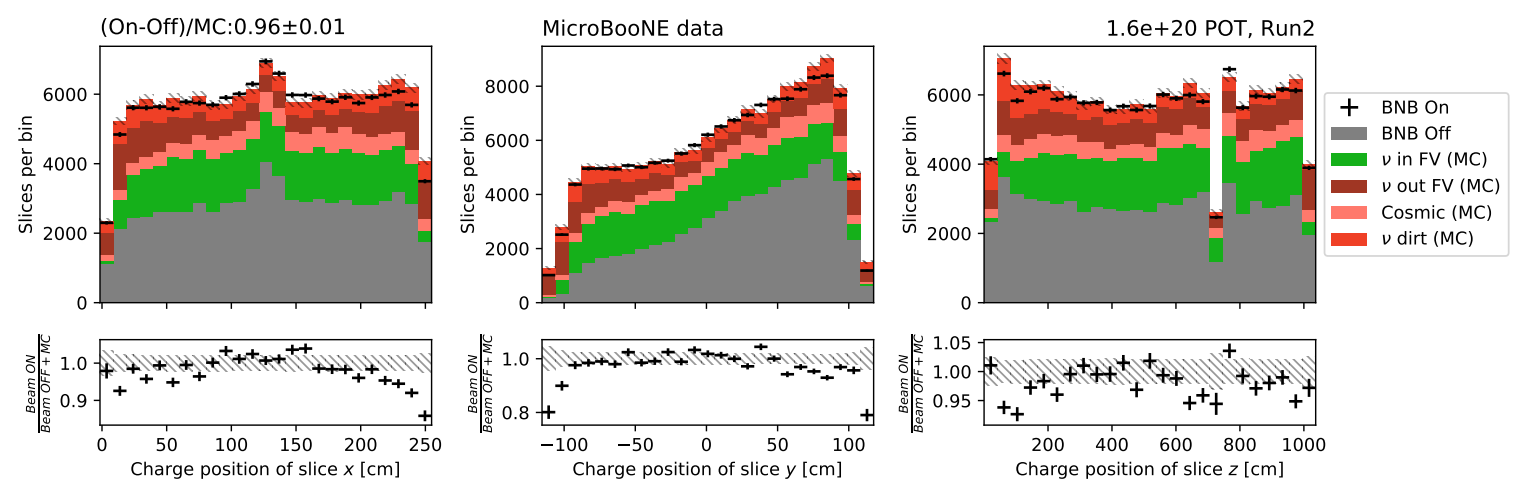

Figure 6.21: Distributions of the charge centre after the NeutrinoID. At this point, no fiducial volume restriction is applied. Comparing the earlier plots in this chapter, the amplification of the green neutrino events becomes apparent. On the middle panel, it can be seen that most of the cosmic backgrounds are located in the upper part of the detector where the cosmic rays enter. On the right panel, the effect of the unresponsive wire region between $675 \mathrm{~cm}$ and $775 \mathrm{~cm}$ is clearly visible.

The slight decrease in efficiency at lower energies is due to reconstruction effects. The performance of the NeutrinoID is disentangled from reconstruction effects in Figure 6.22. Here, the efficiency of the different steps in the NeutrinoID for both the signal categories as a function of the true neutrino energy and $x$ vertex position are shown. Only well-reconstructed 
slices are included. It can be seen that the final NeutrinoID efficiency is above $90 \%$ on average, and well above $85 \%$ at the lowest energies and at the highest $x$-positions.
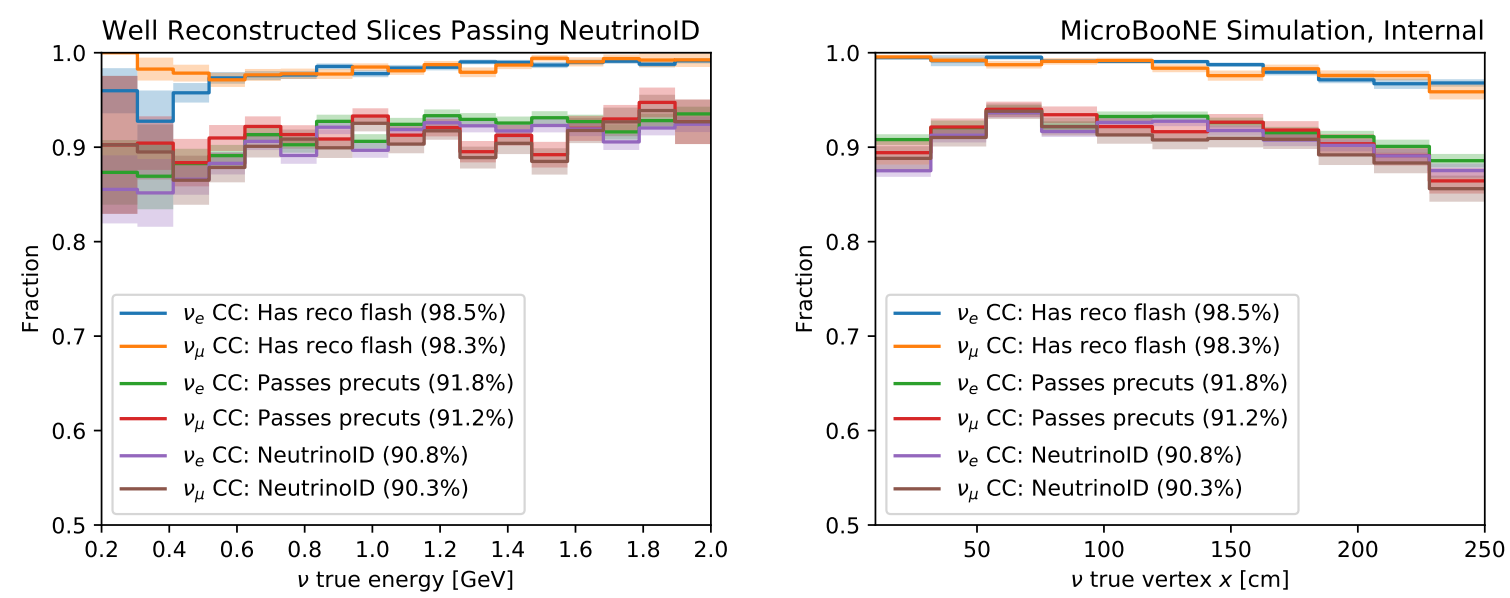

Figure 6.22: NeutrinID efficiency as a function of neutrino energy and vertex $x$ location. The three different steps in the NeutrinoID are given for the two signals: $v_{\mu} \mathrm{CC}$ and $v_{e} \mathrm{CC}$ events. The number is the legend is the total efficiency. It can be appreciated that the dependency of the $x$ location or energy is minimal if the slice is well-reconstructed. The shaded area corresponds to the statistical uncertainties. Only a subset of the available MC is included in these plots.

\section{Continuity over different data-sets}

Due to the data blinding policy in MicroBooNE, the following chapters will be strongly statistical limited. Therefore, it is instructive to take a look at the continuity of the NeutrinoID over an extensive data-set spanning multiple years. The total MicroBooNE data-taking campaign between October 2015 and October 2019 spans four Runs ${ }^{2}$ (see Figure 10.1). The stability of the NeutrinoID was validated on the three first data runs as illustrated in Figure 6.23, including $2.2 \times 10^{20} \mathrm{POT}$, corresponding to approximately one fifth of the complete MicroBooNE data set.

For the purpose of stability testing, a purer neutrino selection is developed on top of the NeutrinoID. This sample enables to look at the amount of reconstructed neutrino interactions per POT in a large data-set spanning three years of data-taking. The selection implements

\footnotetext{
${ }^{2}$ note the difference between DAQ runs that last a maximum of 7 hours, and one-year data-taking runs.
} 

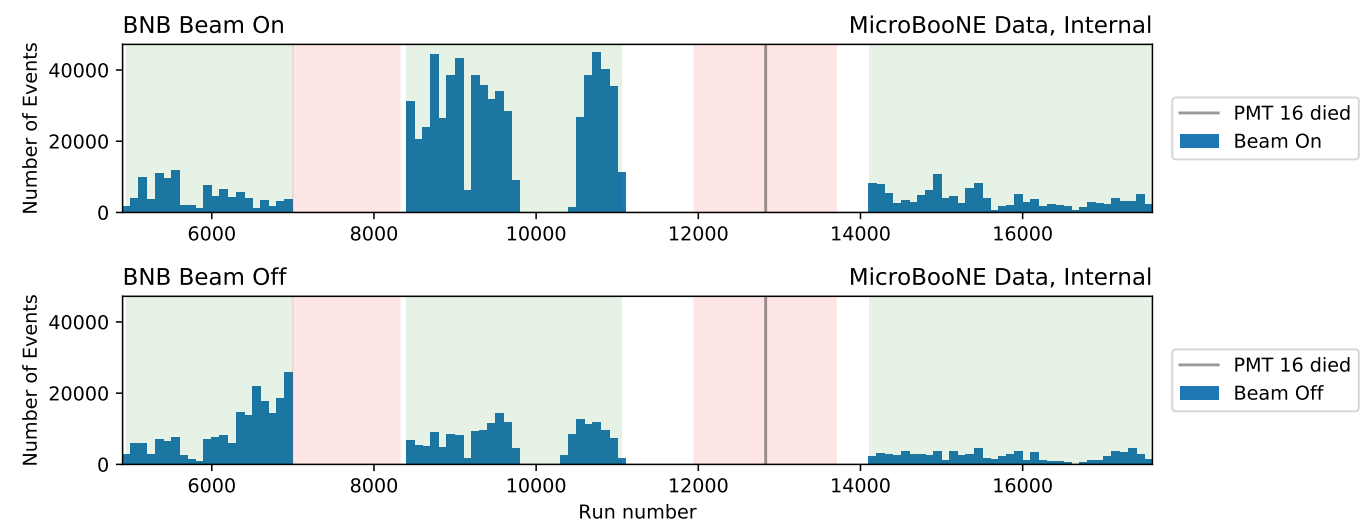

Figure 6.23: Number of events per run. Run numbers in Beam On and Beam Off samples used to validate the NeutrinoID. The gap in the second run period is due to month-long downtime related to high-voltage instabilities.

two additional cuts:

- The centre of deposited charge is required to be in the FV as defined in Figure 6.1.

- The selected neutrino slice should have a topological score larger than 0.15.

After these two additions, the efficiency for $v_{e}$ CC events is $(65.34 \pm 0.30) \%$, and $(65.17 \pm 0.43) \%$ for $v_{\mu}$ CC events. The Beam Off events are supressed by an additional factor 7 . This toyselection enables us to look at the stability of an event sample that is largely dominated by neutrino induced activity.

Different steps described in this chapter are now evaluated as a function of the time the data was collected - represented by the DAQ run number. The result is given in Figure 6.24. The five panels from top to bottom represent:

1. Beam On, Events per POT. The number of events summed for 100 runs and quoted per $1 \times 10^{16}$ protons on target. The large fluctuations beyond the statistical errors are attributed to the lack of beam quality monitoring cuts. These will be included in the following chapters. For the first stage (blue), most of these events are cosmictriggered events. In green, the event rate after the NeutrinoID is given, at this point, neutrino induced events make up a significant part of the passed events. In red, the pure selection passed events are dominated by neutrino induced activity. 
2. Number of passed events per event trigger. As can be seen from the red line, approximately one neutrino induced event takes place for every 750 bunch crossings. After applying beam quality checks, this number is $\mathcal{O}(1 / 600)$.

3. Events per external trigger. This variable should be interpreted as a measure of the Beam Off rejection power.

4. Neutrino induced events per $1 \times 10^{16}$ POT: The beam data subtracted with the cosmic triggered data.

5. Neutrino induced purity. Fraction of passed data events that are neutrino induced, averaged per 100 runs.

It can be seen that the events per trigger, both in Beam On (second panel) and Beam Off (third panel) are decreasing as a function of time. This was anticipated by Figure 3.12, where the decreasing light yield reduces the flash reconstruction efficiency. It can be appreciated that the number of events per POT stays fairly constant within fluctuation. Therefore, it can be concluded that the effect of the reduced light yield over time is of small impact on the neutrino selection performed in this chapter. Nonetheless, if the signal sample is situated at the lowest energies - as is targeted for the LEE analyses - additional validations are needed in later selections steps. For example, one can validate the flash inefficiency by looking at the amount of selected events near the cathode (high $x$ ) as a function of time.

Figure 6.24 validates the stability between the ratio of different stages in the NeutrinoID. 

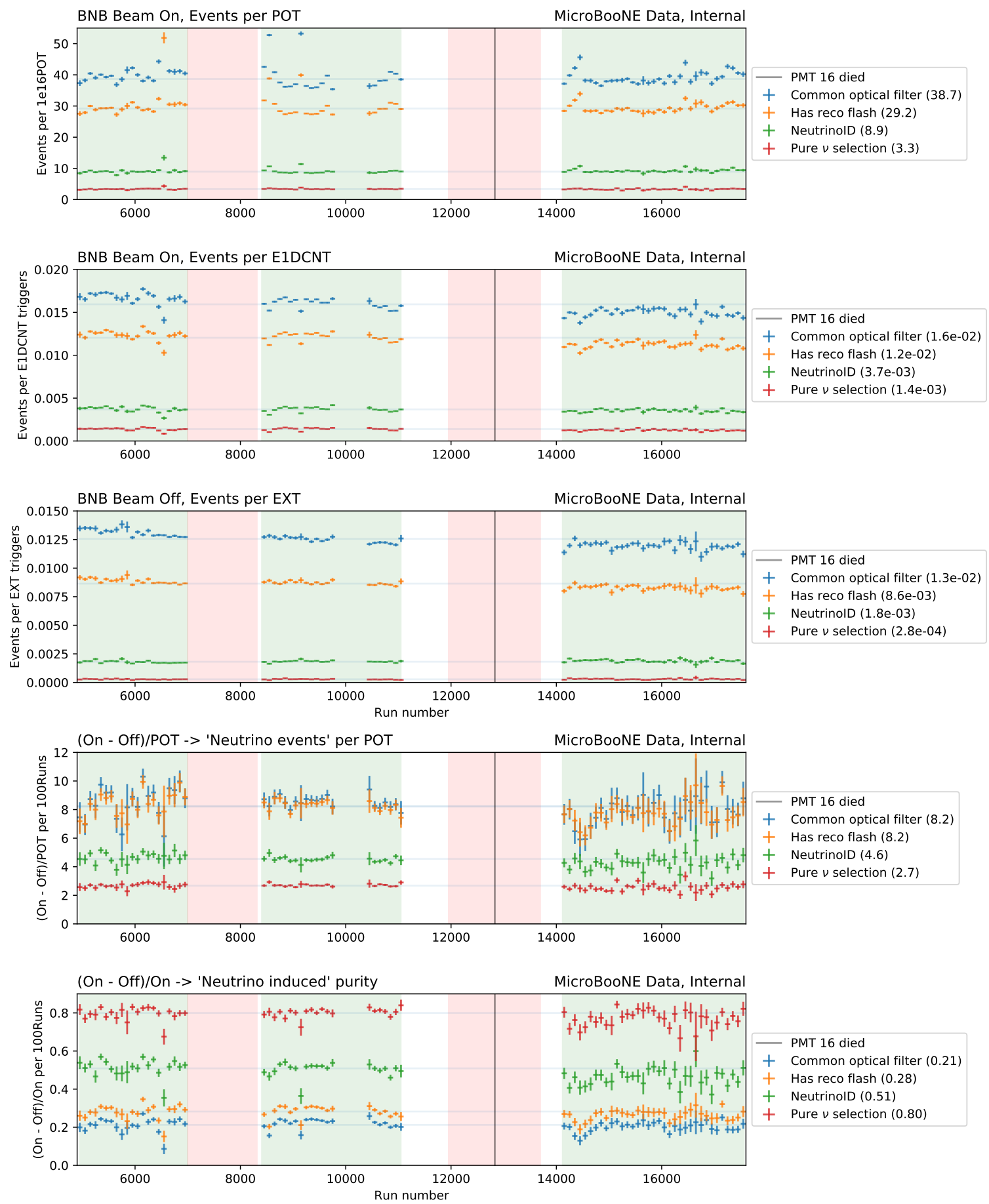

Figure 6.24: Performance and stability of the NeutrinoID over different data-sets. The gap in the second run period is due to high voltage instabilities. The total exposure included is $2.2 \times 10^{20}$ POT. The DAQ run number is given on the horizontal axis and represents time, the bin size is 100 runs per bin. The values in the legend next to the labels are averaged over all data. The details of these plots is given in the text. 


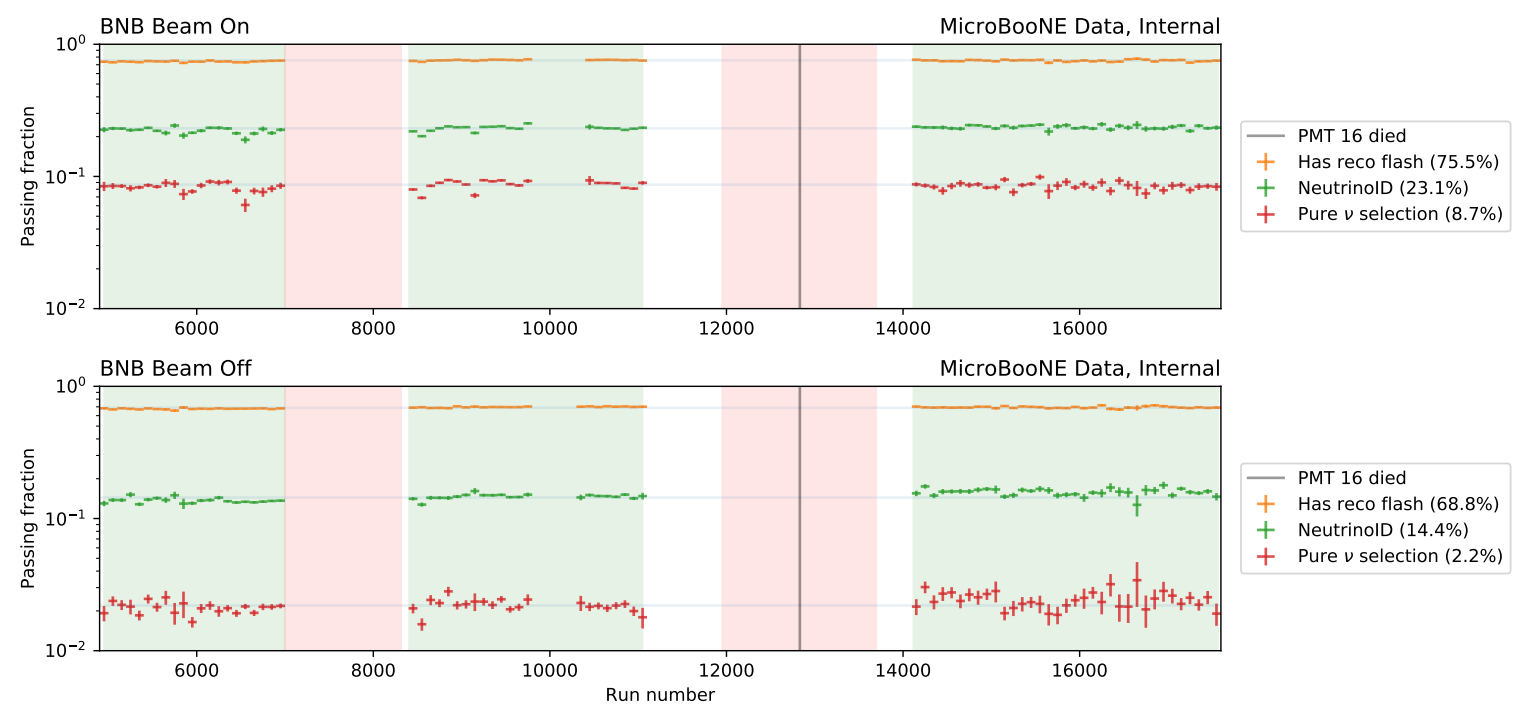

Figure 6.25: Ratio of passing rates in various steps of the NeutrinoID over the data-set. The gap in the second run period is due to high voltage instabilities. The total exposure included is $2.2 \times 10^{20}$ POT. The DAQ run number is given on the horizontal axis and represents time, the bin size is 100 runs per bin. The values in the legend next to the labels are averaged over all data. The details of these plots is given in the text. 


\subsection{Additional cosmic rejection using the CRT}

The Cosmic Ray Tagger (CRT) system was introduced in Section 3.2.3 and partly validated through a cosmic muon measurement in Section 5.4. The main reason for the existence of the muon-tagging system is to further reduce the cosmic neutrino background for contained neutrino events in the MicroBooNE LArTPC. Two techniques that will be used in the next chapters are introduced here.

CRT veto The CRT veto looks for a time coincidence between the scintillation light recorded in time with the $1.6 \mu$ s beam spill - the beam flash - and a CRT hit. If a CRT hit occurs within a $1 \mu$ s of the beam flash, the event is rejected. No constraint on the position of the flash nor on the position of the CRT hit is applied. The distribution of the time differences for the flash and the CRT signal is shown in the left panel of Figure 6.26.
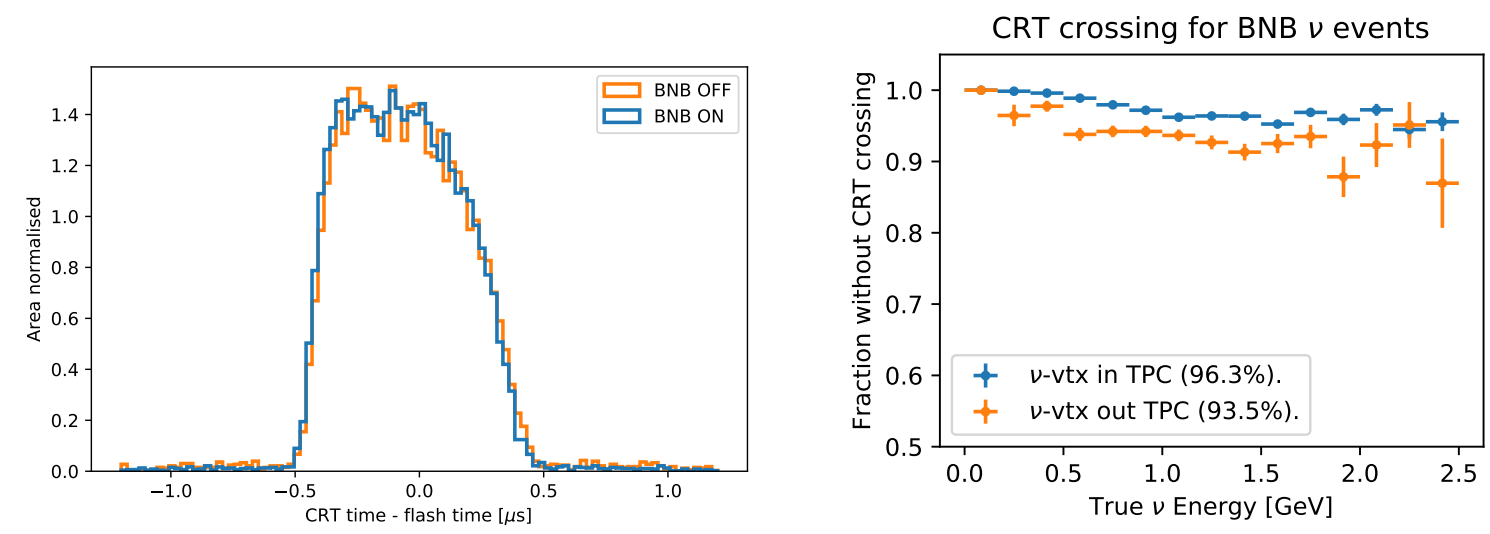

Figure 6.26: (Left) Time difference between the beam flash and the nearest CRT hit. Area normalised for both the Beam On and Beam Off data samples. It can be seen that the resolution of the CRT-flash-matching is $\approx 1 \mu$ s and that the amount of random coincidences - the uniform background - is negligible. (Right) Estimation of the impact of the CRT veto on simulated electron neutrino events as a function of the true neutrino energy. The effect of interest is on events interacting inside the TPC (blue).

The rejection power and efficiency of the CRT veto are calculated using the Beam Off and the simulated BNB intrinsic $v_{e}$ samples, respectively. The fraction of Beam Off events that is rejected is $\approx 59 \%$. The efficiency for $v_{e}$ signal events is $\gtrsim 96 \%$ for all electron neutrino 
energies, raising for compact interactions at low energies (right panel in Figure 6.26). For uncontained $v_{\mu}$ CC events, a large fraction $-\mathcal{O}(15 \%)$ - is rejected by the CRT veto. Furthermore, this technique also rejects a significant proportion of simulated dirt events. The intersection points of simulated particles crossing the CRT planes is given in Figure 6.27

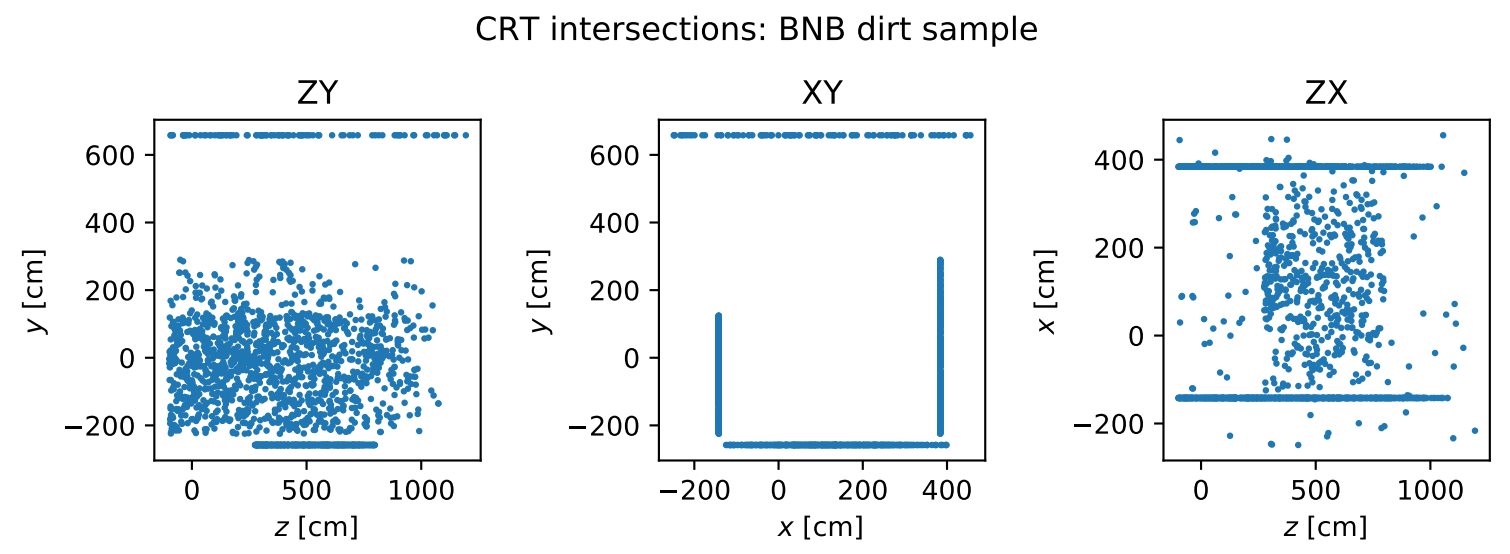

Figure 6.27: CRT intersections as used by the veto to reject simulated neutrino interactions outside the cryostat.

CRT distance tagger The CRT distance tagger builds upon the Pandora neutrino vertex reconstruction and the CRT tagging of TPC tracks. A TPC track is associated with a CRT hit if the track projection onto a CRT panel and a CRT hit are within $15 \mathrm{~cm}$. To perform this association, the track projection to the CRT is calculated under the hypothesis that the associated particle crossed the TPC at the time registered by the CRT hit under consideration. If the minimum distance between the reconstructed neutrino vertex and the nearest track tagged with a CRT hit is less than $10 \mathrm{~cm}$, the event is rejected. An example event tagged by this cut is shown in Figure 6.28. For the CRT distance tagger, the Beam Off passing rate is $\approx 81 \%$, and the $v_{e}$ efficiency $\gtrsim 96 \%$ for all electron neutrino energies. 


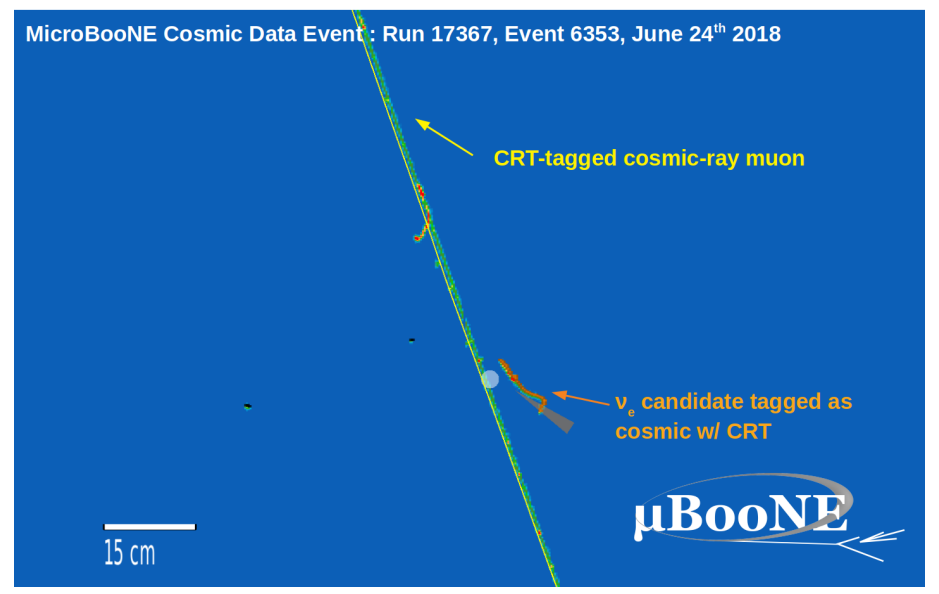

Figure 6.28: Example $v_{e}$ candidate tagged as background by the CRT distance tagger. From the event one can see that the reconstructed electromagnetic shower is incorrectly associated to the incoming muon. 


\section{Charged-Current Muon Neutrino Selection}

This chapter describes the event selection of charged-current muon neutrino interactions in the MicroBooNE LArTPC. Section 7.1 will motivate the importance of charged-current muon neutrino events. The event selection and the muon identification will be the topic of Sections 7.2 and 7.3. The performance and distributions after the selection will be discussed in Section 7.4. The data used in this and the next chapters correspond to the unblinded $4.1 \times 10^{19}$ POT from the first data-taking period and $0.9 \times 10^{19}$ POT with CRT data from the third. Both data-sets were filtered to only contain data passing the beam quality requirements and was recorded during stable detector operating conditions. The errors included in this chapter are statistical only, systematic uncertainties will be discussed in Section 9.1. 


\subsection{Motivation}

In 2019, MicroBooNE published its first two cross-section results using charged-current muon neutrino events. One measured differential cross sections of inclusive charged-current muon neutrino events, demonstrating the neutrino reconstruction and identification capacities of MicroBooNE [88]. The second measured the $v_{\mu} \mathrm{CC} \pi^{0}$ production, confirming the ability to perform electromagnetic shower reconstruction with the detector [89]. Internally, these results were the culmination of the MicroBooNE Monte Carlo Challenge 8. Since then, MicroBooNE has advanced to its final Monte Carlo Challenge iteration, 9. From 8 to 9, major steps in the signal processing, reconstruction framework and detector simulation were overhauled and upgraded. One of those new additions in is the common cosmic rejection and neutrino identification step - the NeutrinoID - as described in Chapter 6. In the next sections a new $v_{e}$ CC event selection will be built on top of those iterations and new tools.

\subsubsection{Inclusive charged-current muon neutrino selection}

Monte Carlo Challenge 9 aims to exploit the fine granularity of the LArTPC and investigate nuclear effects through exclusive neutrino interaction measurements. Most of these studies will use a subset of $v_{\mu} \mathrm{CC}$ events. Section 2.5 introduced a set of mechanisms through which neutrino-argon scattering takes place at the $v$ energies from the Booster Neutrino Beam. The ultimate goal of the MicroBooNE experiment is to study and isolate nuclear effects to improve our understanding and modelling of interactions for current and next generation experiments. Furthermore, the knowledge obtained from this drives the ability to reconstruct the neutrino energy, and therefore the ability to search for new oscillation physics beyond the Standard Model. Several analyses that use the selection described in this chapter are currently in progress:

- $v_{\mu}$ CC $0 p 0 \pi$ : This channel is hard to distinguish from cosmic muons due to the lack of vertex activity. Nevertheless, it is the best way to construct an event sample that is dominated of quasi-elastic interactions.

- Single transverse variables: This set of variables characterise the momentum imbalance 
between the outgoing lepton and the most energy proton in the plane transverse to the neutrino direction. The study of neutrino cross sections as a function of transverse variables provides a powerful handle on theoretically challenging nuclear effects in neutrino interactions [90]. To study transverse variables, a pure $v_{\mu}$ CC N $p$ sample is essential.

- $v_{\mu}$ CC $\pi^{0}: \mathrm{A} \pi^{0}$ can be produced during a resonant neutrino-argon interaction (see Figure 2.12) or through Final State Interactions. These specific production mechanisms enable probing nuclear processes of interest. Furthermore, as will become apparent in Chapter 8 , events with a final state $\pi^{0}$ are the dominant background for $v_{e}$ CC searches and enable us to study low-energy electromagnetic showers through the $\pi^{0} \rightarrow \gamma \gamma$ decay.

\subsubsection{Contained high-purity $v_{\mu} \mathrm{CC}$ selection}

A $v_{\mu}$ CC sample can also be used to constrain the systematic uncertainties in the $v_{e}$ CC analysis. Such a constraint is possible because the $v_{\mu} \mathrm{s}$ and $v_{e} \mathrm{~s}$ of the BNB are subject to common, correlated uncertainties. The flux, cross-section models and detector uncertainties will be further discussed in Section 9.1. The $v_{e}$ selection is subject to higher uncertainties due to its lower statistics: the $v_{\mu}$ channel benefits from two orders-of-magnitude larger absolute flux (see Table 3.1). Therefore, selecting $v_{\mu}$ CC interactions with high statistics can be exploited to constrain the uncertainties on the $v_{e}$ predicted event rate.

This specific selection which is a sub-selection of the aforementioned inclusive selection, prioritises the low-energy region that is the interest to the LEE analyses. To attain this goal, the lepton selection will be limited to fully-contained muons. Additionally, the CRT veto will be used to reject approximately $2 / 3$ of the remaining Beam Off background events (see Section 6.5). This selection is developed in extensively motivated in Section 7.4.2, and used as a data-driven constraint in extensively motivated in Section 9.2, 


\subsubsection{Selection strategy}

Of all MicroBooNE recorded events triggered by the BNB, only one in approximately 600 contains a neutrino interaction in the TPC. Furthermore, every recorded event - with or without neutrino interaction - contains approximately 15 cosmic-muon interactions. The selection must therefore reject cosmic activity and identify the muon produced by the chargedcurrent interaction.

The flowchart in Figure 7.1 introduces the strategy followed. At the preselection stage, Section 7.2, cosmic rejection is obtained by combining the tools introduced in Chapter 6 (the NeutrinoID) with additional cuts to further reject cosmic activity. In Section 7.3, the muon identification is described. The combination of the preselection and the muon requirement lead to the inclusive charged-current muon neutrino selection (Section 7.4.1). This selection is further expanded in Section 7.4.2 to achieve a higher purity and better muon momentum resolution. In Section 7.4.3, some examples of exclusive selections are given.

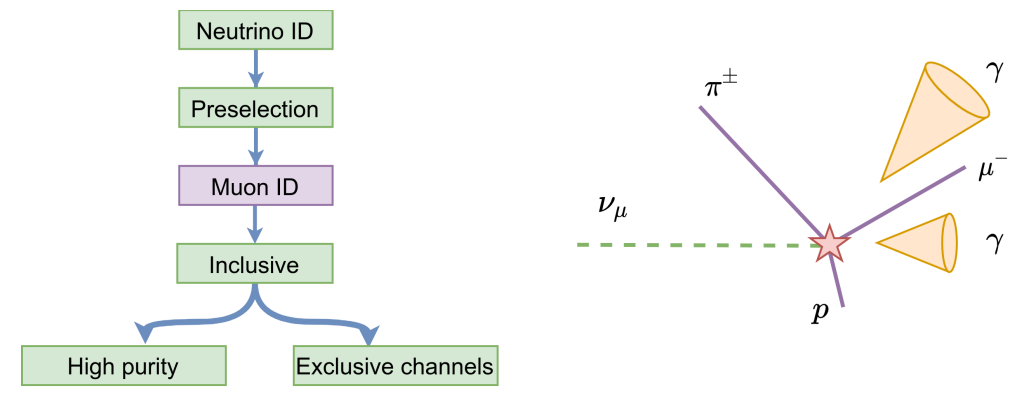

Figure 7.1: (Left) Flowchart indicating the different steps in the $v_{\mu}$ CC selection. (Right) A hypothetical $v_{\mu}$ CC event with multiple reconstructed showers and tracks. At the preselection stage, the largest object in the event is required to be track-like (purple). On all tracks, muon identification is performed. If an event passes the preselection and a muon is found, it is selected by the $v_{\mu} \mathrm{CC}$ inclusive selection. The high-purity selection requires additional muon containment and uses the CRT. The inclusive selection is used in Section 7.4.3 as a basis to study more exclusive channels. 


\subsection{Event preselection}

In the data/MC comparisons throughout this chapter, the simulated samples will be split up in the following categories:

$-v_{\mu}$ CC. Signal events as introduced in Section 6.1. Subdivided in three channels:

- $0 p$ : No protons or pions in the final state above $40 \mathrm{MeV}$ kinetic energy. The signature is a single muon track.

- $0 \pi \mathrm{N} p$ : At least one proton with $E_{k i n}(p)>40 \mathrm{MeV}$ in the final state but no pions above threshold. $\mathrm{N}>0$.

- $\mathrm{M} \pi \mathrm{N} p$ : At least one pion with $E_{k i n}(\pi)>40 \mathrm{MeV}$, no restrictions on the number of protons. $\mathrm{M}>0$.

The event categories defined above are used in the numerator to obtain the selection efficiency for each channel later in this chapter.

- $v_{\mu}$ NC events. This category can be mistaken for charged-current events if a charged pion or a long proton track is present.

$-v_{e}$. Intrinsic component of the BNB.

- Cosmic. Events that contains a simulated neutrino, but the reconstructed neutrino activity consists of $50 \%$ or more out of cosmic charge deposits.

- Out of FV. Events that contain a simulated neutrino with interaction vertex which is inside of the cryostat, but outside of the fiducial volume.

- Out of Cryo. This is equivalent to the $v \operatorname{dirt}(M C)$ category, introduced in the previous chapter. Simulated neutrino events with the interaction vertex outside of the MicroBooNE cryostat.

Fiducial volume In Figure 7.2 the vertex position of the neutrino candidates is given in all three dimensions of the TPC. The fiducial volume as introduced in Figure 6.1 is applied to 
reject the background events from the edges of the detector. In the left panel of the figure, it is apparent that the dirt events occur mainly in the very front of the detector.

Apart from the vertex, the start points of all reconstructed particles associated with the neutrino vertex are required to be contained in a more relaxed fiducial volume. This containment volume is defined by borders of $5 \mathrm{~cm}, 6 \mathrm{~cm}$ and $10 \mathrm{~cm}$ in the respectively $x, y$ and $z$ direction from the edges of the TPC.
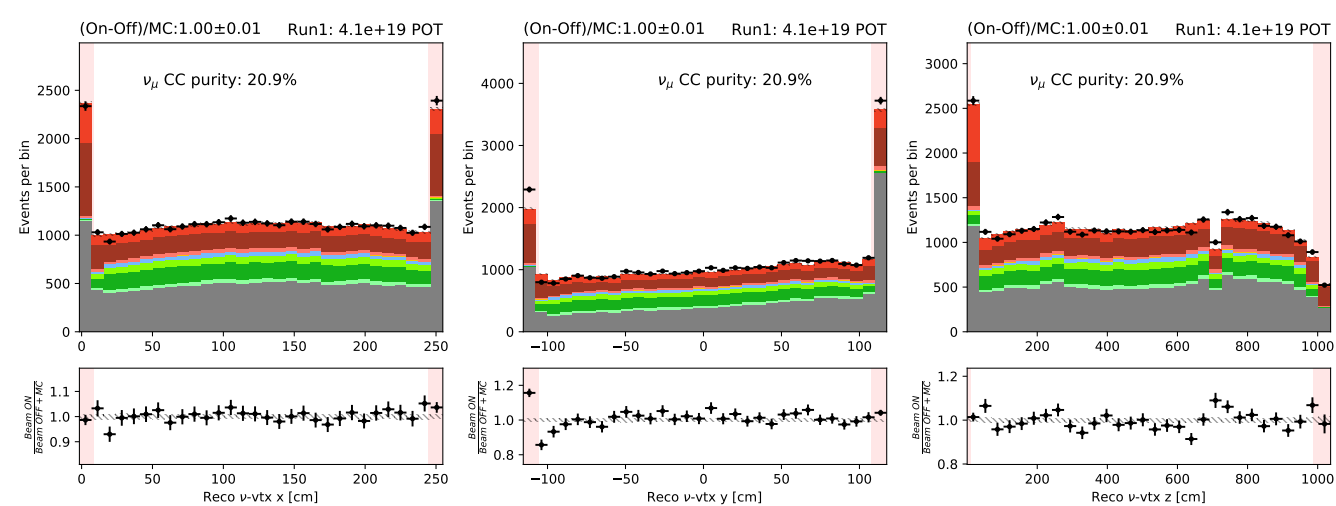

+ BNB On: 35809

$v_{\mu}$ CC Op: 1005 .

$\nu_{\mu}$ CC OrNp: 4589

$v_{\mu}$ CC MnNp: 1903

$v_{\mathrm{e}}: 56$.

Cosmic: 984.

Out of FV: 6543

Out of Cryo: 3486

Figure 7.2: Reconstructed interaction vertex positions in $x$ (left), $y$ (middle), $z$ (right). The red shaded areas correspond to the region removed after fiducialisation. Events selected by the NeutrinoID are shown.

As the next step in the selection, the final state particle with the largest amount of hits in the event is required to be track-like, see Figure 7.1. This requirement reduces the $v_{e}$ contribution by a factor of three, but also rejects a third of the neutral-current events, especially the NC $\pi^{0}$ channel with photon showers. The downside of this requirement is that it also affects the $v_{\mu}$ CC $\pi^{0}$ efficiency, especially at low energies. Nevertheless, the cut is essential to enable the described selection to comply with the MicroBooNE blindness criteria. These criteria enable the opening of extensive data-set on the premise that it is demonstrated that the included number of $v_{e}$ CC events is sufficiently small.

Additional cosmic rejection Figure 7.3 shows two cuts aimed to reduce cosmic activity further. To attain this goal, the topological score of the neutrino candidate slice is required to be above 0.1. An additional requirement on the cosmic impact distance is introduced. This 
variable corresponds to the closest distance between the neutrino vertex and space points associated with tracks flagged as cosmic activity. This distance is required to be at least $10 \mathrm{~cm}$. While the cut on the topological score is extremely efficient in the rejection of Beam Off events, it removes a significant fraction of the low-energy $v_{\mu}$ CC $0 p$ signal. This trade-off in cosmic rejection and low-energy events was anticipated in Figure 6.18. The cuts described in this section are summarised in Table 7.1.
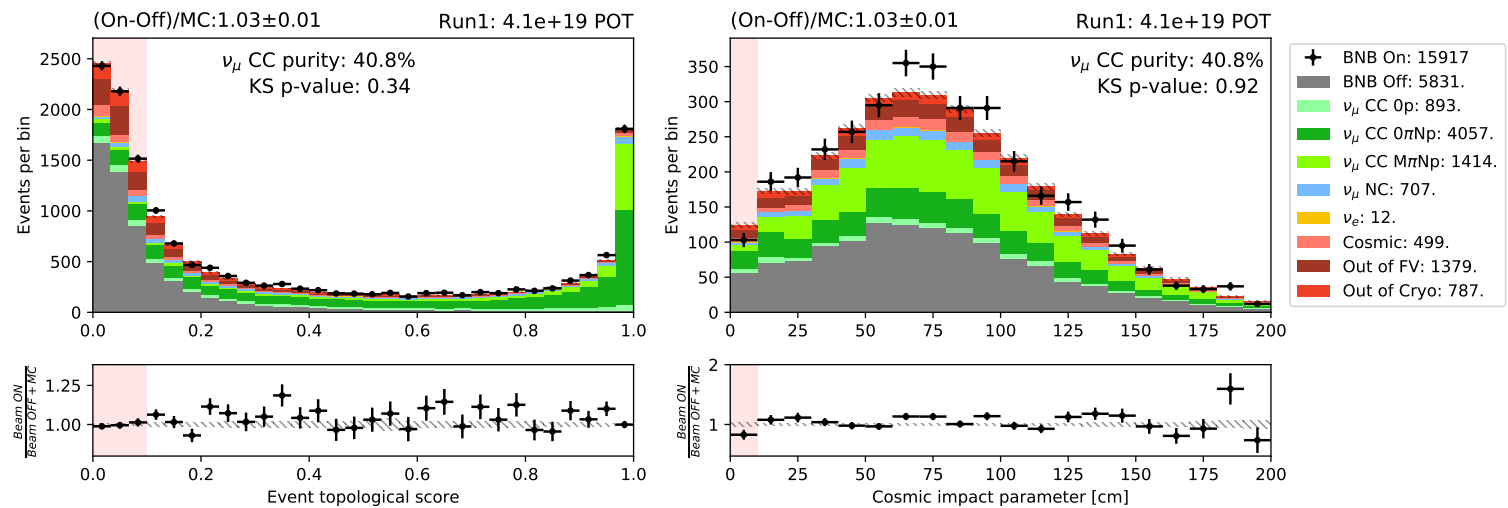

Figure 7.3: The event topological score as described in Section 6.3.1 (left) and the cosmic impact distance as described in the text (right). Events in the shaded red regions are rejected. Both cuts are aimed to reject remaining Beam Off background.

Table 7.1: Event selection requirements for the $v_{\mu}$ CC selection. The left column lists the main goal of certain criterion. Note that most cuts are multi-functional.

\begin{tabular}{|c|c|}
\hline Cut goal & Cut definition \\
\hline \hline & Selected by the NeutrinoID \\
Cosmic rejection & Reconstructed neutrino vertex is in FV \\
& Start point of particles is contained \\
& Topological score $>0.1$ \\
& Cosmic impact distance $>10 \mathrm{~cm}$ \\
\hline$v_{e} \&$ NC rejection & Dominant final state particle is track-like \\
\hline
\end{tabular}




\subsection{Muon Identification}

The next step of the selection aims to tag the muon. This is needed to look at the $v_{\mu} \mathrm{CC}$ lepton kinematics, but also to reject neutral-current events without muons in the final state. Note that throughout this section, all immediate neutrino daughters will be considered. The event does get discarded if no suitable muon candidate is found.

The first two cuts are illustrated in Figure 7.4 and aim to remove electromagnetic shower activity by using the track score, and making sure the muon is attached to the neutrino vertex by requiring the track start point and the reconstructed neutrino vertex to be within $4 \mathrm{~cm}$. The combination of these cuts excludes the possibility for mis-reconstructed events often with cosmic impurities or near unresponsive wire regions - to be selected. The next step is to identify the muon from the protons produced in interactions.
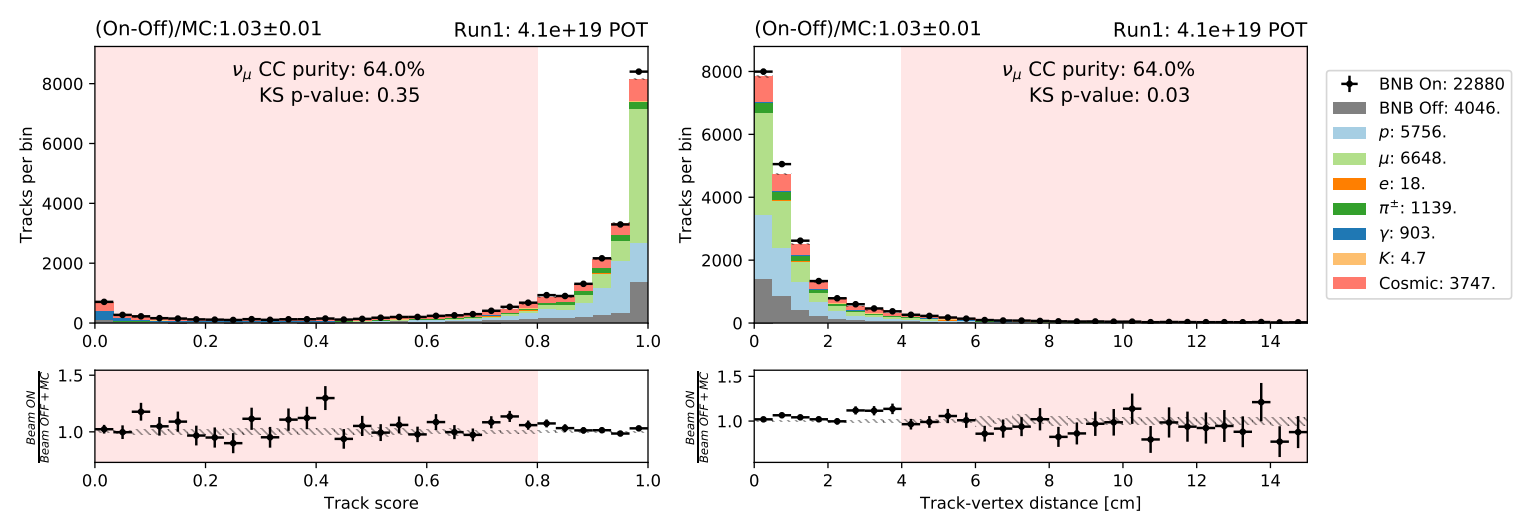

Figure 7.4: The track score as described in Section 4.2.3 (left) and the distance between the neutrino vertex and the start of the track (right). Note that the legend refer to different particle types. Histograms are filled for every track associated with the neutrino.

\subsubsection{Proton-muon separation using the likelihood ratio}

For track-like particles, the Particle Identification (PID) is performed by looking at the profile of the deposited energy per unit length $(\mathrm{d} E / \mathrm{d} x)$. In this thesis, track PID is limited to a binary classification problem, i.e. how to distinguish protons from muons. We disregard further classification because charged pion tracks are challenging to distinguish from muons if using only calorimetry information. Furthermore, kaons are rarely produced in 
neutrino interactions at the neutrino energies of interest for MicroBooNE. Additional information, such as possible hadronic re-interactions or Michel electrons, can be powerful to perform additional particle identification but is beyond the scope of this analysis.

The expected distribution of the $\mathrm{d} E / \mathrm{d} x$ is modelled for each particle type and for each wire plane, as a function of two variables:

- residual range $(r r)$ : The distance of a given trajectory point from the end of the track, measured along the track trajectory.

- pitch: the distance between two subsequent wires along the projected particle trajectory. If the particle travels orthogonal to the wire plane, the pitch equals the wire spacing of $3 \mathrm{~mm}$. This is the length over which the charge is measured to calculate the $\mathrm{d} E / \mathrm{d} x$ for a given trajectory point.

The probability to measure a certain energy loss per $\mathrm{cm}$ is expressed as:

$$
P(\mathrm{~d} E / \mathrm{d} x \mid \text { type, plane, } \mathrm{rr}, \text { pitch }) \text {. }
$$

The expected distribution of the energy deposition is modelled for each plane independently, and for the two kinds of particles under study, protons and muons.

Multiple effects enter in the model of this distribution. The first effect is what we want to leverage: the average $\mathrm{d} E / \mathrm{d} x$ at a given residual range depends on the particle's mass, as seen by integrating the Bethe-Bloch function for different masses. Secondly, fluctuations of $\mathrm{d} E / \mathrm{d} x$ depend on the pitch. These fluctuations are intrinsically stochastic: the longer the length over which the charge is averaged, the smaller the fluctuations. Furthermore, detector effects such as recombination, signal deconvolution, and hit reconstruction add a non-linear response and smearing; this response depends on both the true deposited charge and the pitch, and cannot be adequately described by an analytic model. For this reason, the expected distribution of $\mathrm{d} E / \mathrm{d} x$ is built starting from the simulation.

The model for $\mathrm{d} E / \mathrm{d} x$ is built by considering well reconstructed tracks ${ }^{1}$, contained within

\footnotetext{
${ }^{1}$ In this section, a track is deemed well reconstructed if its completeness and purity are both above $90 \%$
} 
a fiducial volume, and backtracked to protons and muons. The binning of the probability density function is:

$-\mathrm{d} E / \mathrm{d} x\left[\mathrm{MeV} \mathrm{cm}^{-1}\right]:$

$[0,0.5,1,1.5,2,2.5,3,3.5,4,4.5,5,5.5,6,6.5,7,7.5,8,9,10,12,15,20,25,30,35,40,45,50]$

- residual range [cm]: $[0 ., 2,4,7,10,15,20,30,50,100,300,2000]$

- pitch [cm]: $[0.3,0.6,1,1.5,3,30]$

Two examples of the probability density function are provided in Figure 7.5, for two different bins in residual range and pitch.
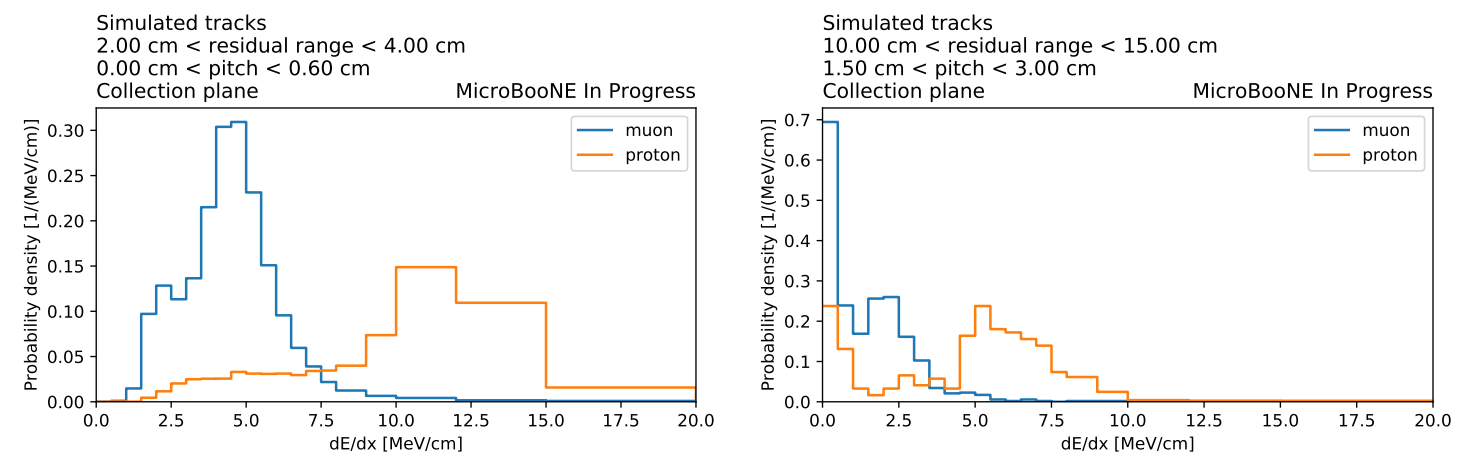

Figure 7.5: Comparison of the expected distribution of $\mathrm{d} E / \mathrm{d} x$ for muons (blue) and protons (orange), for two different bins in residual range (left) and pitch (right). Figure courtesy of Nicolò Foppiani.

For a given track, we consider all three planes. For each plane, the $\mathrm{d} E / \mathrm{d} x$, residual range, and pitch are used to compute the likelihood of each particle type,

$$
\mathcal{L}(\text { type } \mid \text { plane, } \mathrm{d} E / \mathrm{d} x, \mathrm{rr}, \text { pitch })=\prod_{i=1}^{N} P\left(\mathrm{~d} E / \mathrm{d} x_{i} \mid \text { type, plane, } \mathrm{rr}_{i}, \text { pitch }_{i}\right)
$$

where the index $i=1, \ldots, N$ runs over each hit for the considered plane. The combination of the three planes is now straightforward by multiplying the three likelihoods corresponding 
to the $U, V$ and $Y$ wire-planes:

$$
\mathcal{L}(\text { type } \mid d E / d x, \text { rr, pitch })=\prod_{i=1}^{3} \mathcal{L}(\text { type } \mid \text { plane, } \mathrm{d} E / \mathrm{d} x, \mathrm{rr}, \text { pitch })
$$

In order to perform the classification task, the likelihood-ratio test is chosen:

$$
\mathcal{T}(\mathrm{d} E / \mathrm{d} x, \mathrm{rr}, \text { pitch })=\frac{\mathcal{L}(\text { muon } \mid d E / d x, \text { rr, pitch })}{\mathcal{L}(\text { proton } \mid \mathrm{d} E / \mathrm{d} x, \mathrm{rr}, \text { pitch })}
$$

The likelihood ratio, as defined above can be proven to be the most powerful statistical test, i.e. the one with the smallest mis-identification rate for any given value of the efficiency.

The resulting logarithm of the likelihood ratio is applied on the tracks passing the previous steps described in this chapter, alongside with a track length cut of $10 \mathrm{~cm}$. The data/MC comparisons and cut criterion are given in Figure 7.6. It can be seen that the described method yields excellent separation between protons and muons. This cut is complementary with the length cut of $10 \mathrm{~cm}$ which excludes the majority of the remaining Beam Off events.
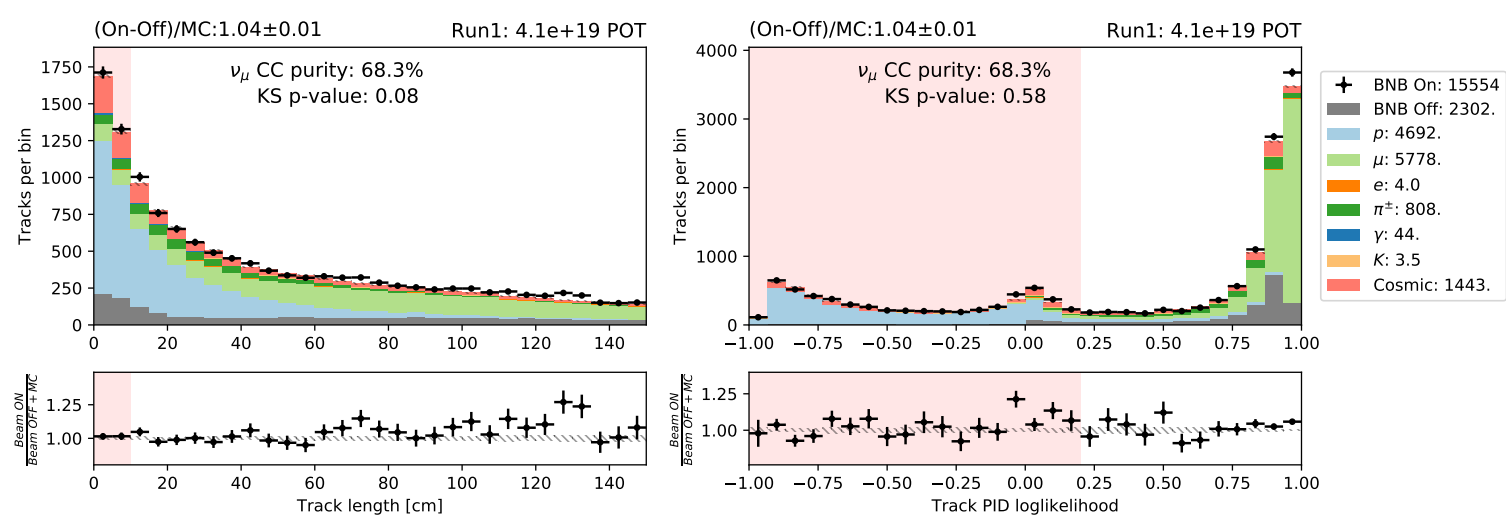

Figure 7.6: The track length (left) and the track log-likelihood PID (right). Note that the legend refer to different particle types. Histograms are filled for every track associated with the neutrino passing the cuts illustrated in Figure 7.4.

The track length requirement of a muon candidate corresponds effectively to a momentum requirement as is illustrated in Figure 7.7. It can be seen that this $v_{\mu} \mathrm{CC}$ selection is appropriate for muons with a momentum above $0.11 \mathrm{GeV} / \mathrm{c}$, or equivalent, a kinetic energy of $48 \mathrm{MeV}$. We remark that this threshold is higher than the $20 \mathrm{MeV}$ threshold decided in the 
signal definition of $v_{\mu} \mathrm{CC}$ events (see Section 6.1).

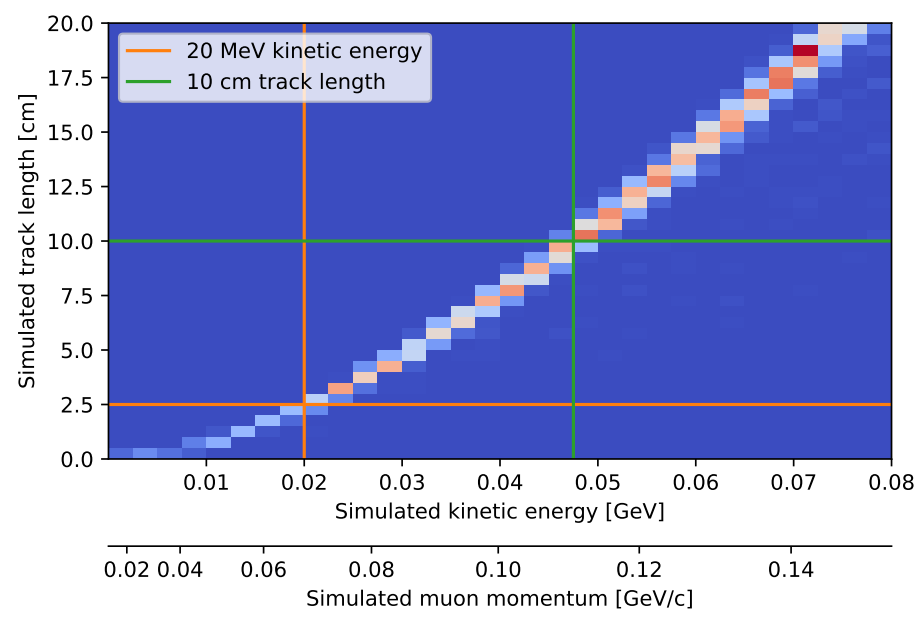

Figure 7.7: Relation between the muon range and its kinetic energy and momentum. The colour represents the number of events and the histogram is filled for simulated muon tracks. Although the signal definition includes all muons with a kinetic energy above $20 \mathrm{MeV}$ (orange lines), the cut on the muon candidate track length of $10 \mathrm{~cm}$ (green lines) corresponds to an effective $E_{k i n}(\mu)$ of $48 \mathrm{MeV}$ or equivalent, $0.11 \mathrm{GeV} / \mathrm{c}$.

Table 7.2: Muon identification requirements for the $v_{\mu}$ CC selection.

\begin{tabular}{|c|c|}
\hline Cut goal & Cut definition \\
\hline \hline Electromagnetic shower rejection & $\begin{array}{c}\text { Track score }>0.8 \\
\text { Track vertex distance }<4 \mathrm{~cm}\end{array}$ \\
\hline Muon-proton separation & $\begin{array}{c}\text { Track length }>10 \mathrm{~cm} \\
\text { Track PID likelihood }>0.2\end{array}$ \\
\hline
\end{tabular}

Table 7.2 summarises the muon identification criteria. For the events that pass the selection cuts discussed in Section 7.2, most of the events have exactly one muon candidate. In $8.9 \%$ of the $v_{\mu}$ CC events, no viable muon candidate is found and the event is rejected. In $13.8 \%$ of the cases, two viable muon candidates are found and in a sub-percent fraction, there can be 3 or more. In this scenario, the muon candidate with the highest muon PID likelihood is tagged to be the muon. The muon identification performance is $95.0 \%$, as will be further discussed by means of Figure 7.11. If the muon tagging failed - which happens most often at the lowest muon momenta - in $3.5 \%$ of the cases, a charged pion is interpreted as the muon, and in $1.3 \%$ of the cases, it is a proton. 


\section{4 $\quad v_{\mu}$ CC selection performance}

\subsubsection{Inclusive selection}

The overall efficiency of the inclusive $v_{\mu}$ CC selections is $(53.35 \pm 0.12) \%$, where the denominator follows the signal definition as introduced in Section 6.1. The overall purity, including cosmic backgrounds, of the selection is $65.5 \%$. The signal efficiency can now be split up into the four different interaction modes (Section 2.5) or into the different final state topologies of interest (beginning Section 7.2). Figure 7.8 shows the efficiency in the different signal sub-categories where the efficiency is similar. However, the efficiency for Deep Inelastic Scattering events is lower. This is explained by taking into account the significant fraction of energy that is lost by nuclear processes in these interactions, therefore reducing the visible deposited energy in the interaction.
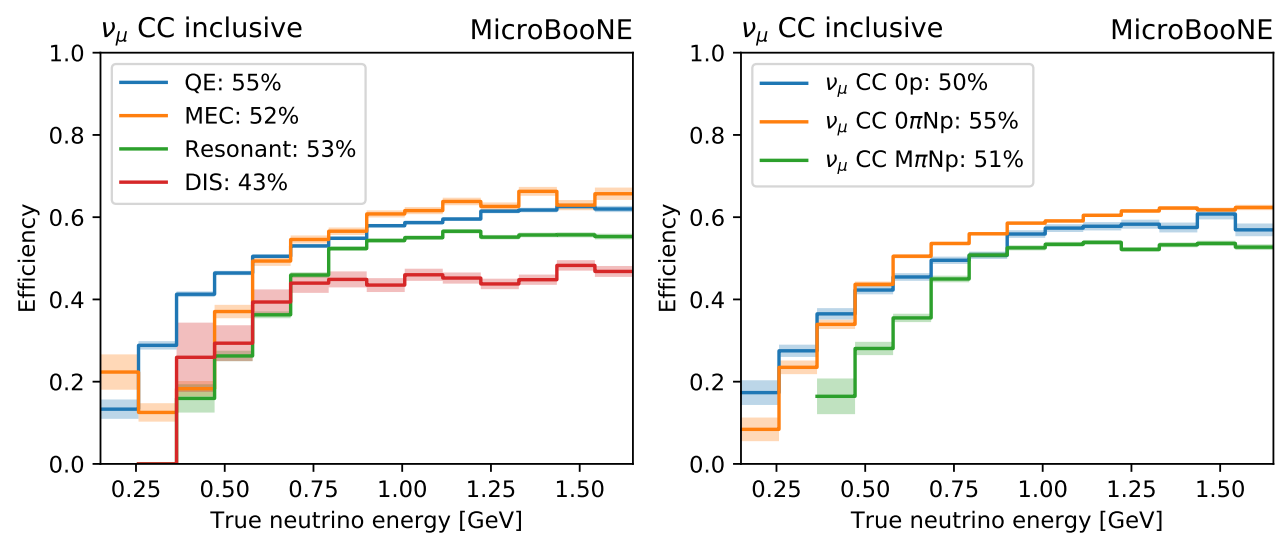

Figure 7.8: Efficiency of the inclusive $v_{\mu} \mathrm{CC}$ selection for different interaction modes (left) and channels (right) as a function of the simulated neutrino energy. The range of the horizontal axis $0.15 \mathrm{GeV}$ to $1.65 \mathrm{GeV}$. The overall efficiency is $(53.4 \pm 0.1) \%$. The mean efficiency is quoted in the legend.

The efficiency should be considered only above $\approx 150 \mathrm{MeV}$. This is because for $v_{\mu}$ CC events, there is the fundamental threshold to produce a final state muon of $106 \mathrm{MeV}$. Additionally, this selection targets muon tracks with a length of $>10 \mathrm{~cm}$, which corresponds to a minimum kinetic energy of $48 \mathrm{MeV}$ in liquid argon. At the very lowest energies, where the events are dominated by Quasi-Elastic scattering - see Figure 3.16 - the selection efficiency 
is around $20 \%$. From $\approx 750 \mathrm{MeV}$, the efficiency reaches a plateau of roughly $60 \%$. The overall efficiency is shaped by the different steps in the selection procedure: The NeutrinoID (17\% signal reduction), events selection (Table 7.1, $20 \%$ ) and the muon identification (Table $7.2,9 \%$ ). The underlying reason of the signal loss can most often be traced back to unresponsive wire zones, masking either the interaction vertex region or breaking up the muon track.

It is important to re-iterate here that the denominator in the efficiency, as described by the signal definition in Section 6.1 is kept exceptionally unrestricted. The fiducial volume (Figure 6.1) including the unresponsive wire regions (Figure A.1), the lack of a containment requirement for the end point of the final state particles and the low muon kinetic energy threshold of $20 \mathrm{MeV}$ all contribute to the reduced efficiency. Nevertheless, since this selection acts as the starting point of a wide variety of many constrained/exclusive selections, it was chosen to maintain the definition of efficiency as inclusive as possible.

Figure 7.9 shows the number of tracks at the vertex - including the tagged muon track - and the muon candidate angle with respect to the beam direction, $\theta$ (see Figure 3.6). These plots present the variety of final state topologies and the coverage of the angular phase space of the selected events.
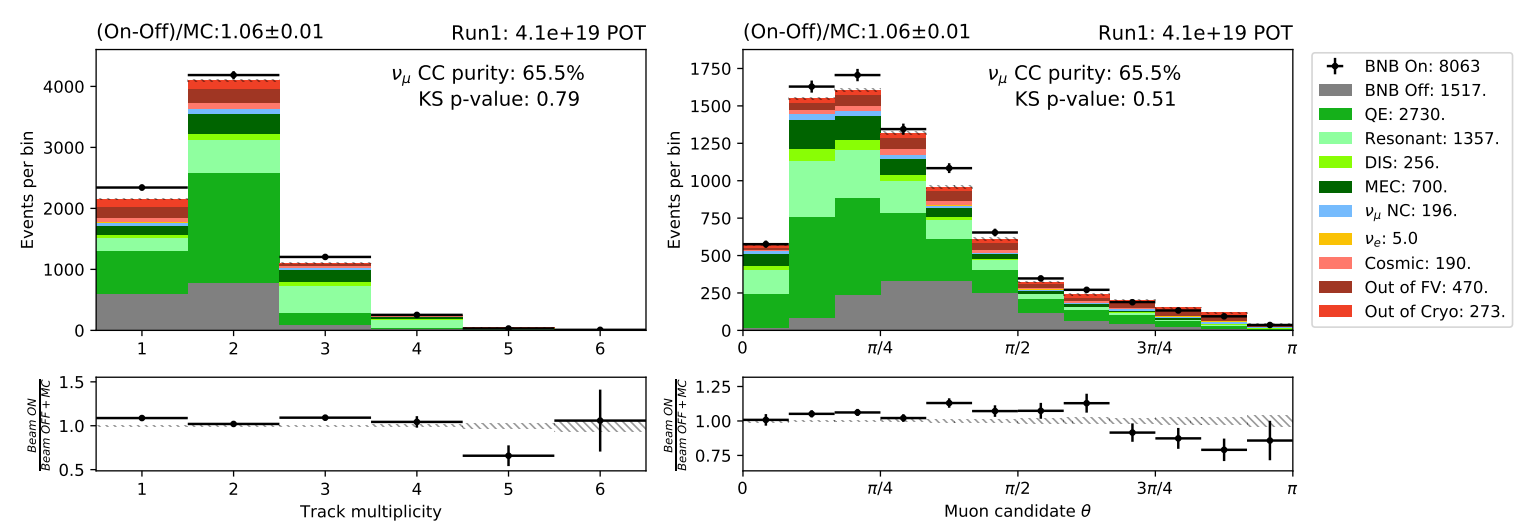

Figure 7.9: Track multiplicity and muon candidate angle w.r.t the beam direction $(\theta)$ for the inclusive $v_{\mu}$ CC selection. The $v_{\mu}$ CC signal events are sub-divided by interaction mode. 


\subsubsection{Contained selection}

As motivated in Section 7.1, we will now further sub-select $v_{\mu} \mathrm{CC}$ events to enhance the purity. Two additional requirements are applied.

- The track identified as the muon candidate has to be contained.

- The CRT tools - as described in Section 6.5 - are applied to achieve a further reduction of the external background.

During the first two years of data-taking, the CRT system was in development and was not fully operational. Therefore, the use of the CRT tools will be demonstrated only for MicroBooNE's third run period, corresponding to $9 \times 10^{18}$ POT. The two additional requirements go hand in hand. The CRT-veto removes a significant amount of $v_{\mu} \mathrm{CC}$ events with uncontained muons, since the exiting muons can trigger the CRT. By requiring muon containement in the selection, this is no longer an issue.

The efficiency for selecting contained $v_{\mu}$ CC events is $(20.2 \pm 0.1) \%$ and is presented as a function of energy in Figure 7.10. The corresponding purity is $76.2 \%$. At the energies of the BNB $v_{\mu}$ CC events, most muons travel several meters inside the liquid argon before stopping. Therefore the majority - approximately $60 \%$ of the muon tracks - is uncontained. The denominator of the efficiency includes both contained and uncontained $v_{\mu}$ CC events, explaining the drop in efficiency compared with the inclusive selection in the previous section.

Figure 7.11 compares the efficiency, muon identification performance and purity of both the inclusive and the contained selections. It can be seen that at low muon energies - when most muons are contained - the performance is highly similar. At the higher energies, the contained requirement rejects most $v_{\mu} \mathrm{CC}$ events and the performance of the latter deteriorates. The underlying cause is that containment is only required for the muon candidate track. The actual true muon in those events with a high true muon energy is very likely to be exiting the detector at these energies $(\gtrsim 1 \mathrm{GeV})$. Therefore, the muon is often not contained at the truth level, and another track in the event is mis-identified as the muon. 

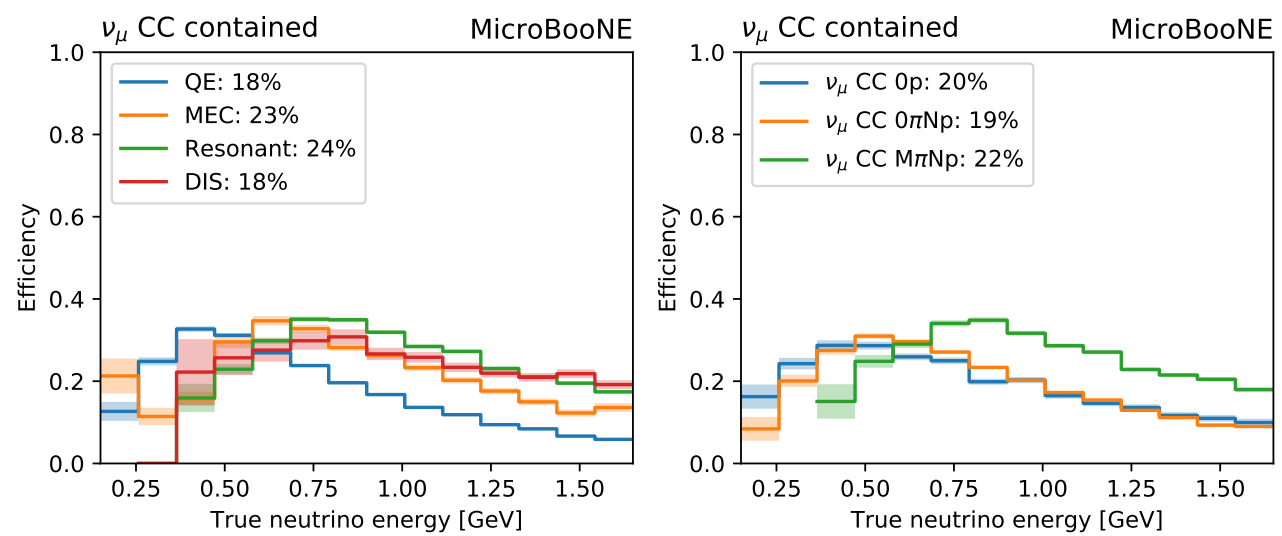

Figure 7.10: Efficiency of the contained $v_{\mu} \mathrm{CC}$ selection for different interaction modes (left) and channels (right) as a function of the simulated neutrino energy. The range of the horizontal axis $0.15 \mathrm{GeV}$ to $1.65 \mathrm{GeV}$. The overall efficiency is $(20.2 \pm 0.1) \%$.

\section{Muon momentum}

The main purpose of the contained selection, besides the improved purity, is the momentum resolution. For contained events, three methods for momentum calculation can be used:

- Calorimetry: From the relativistic energy-momentum equation we find:

$$
p_{\mu}=\sqrt{\left(\frac{E_{\mu}}{c}\right)^{2}-\left(m_{\mu} c^{2}\right)^{2}} .
$$

Where substituting $E_{\mu}=m_{\mu} c^{2}+E_{k i n, \mu}$ gives:

$$
p_{\mu}=\sqrt{\left(\frac{E_{k i n, \mu}}{c}\right)^{2}+2 m_{\mu} E_{k i n, \mu}}
$$

The kinetic energy of the muon can be approximated by the visible calorimetric energy. This methods is of course restricted to contained muons only. Furthermore, due to unresponsive wire regions and angular dependency on the charge calibration - see Appendix A - the obtained energy resolution of this method is limited ( $~ 15 \%$ ) [91].

- Range-based momentum: The Bethe-Bloch equation describes the energy loss of muon traversing liquid argon [12]. This energy loss is dependent on the medium, particle type and its instantaneous energy. The range $R$ a muon travels before coming to a 

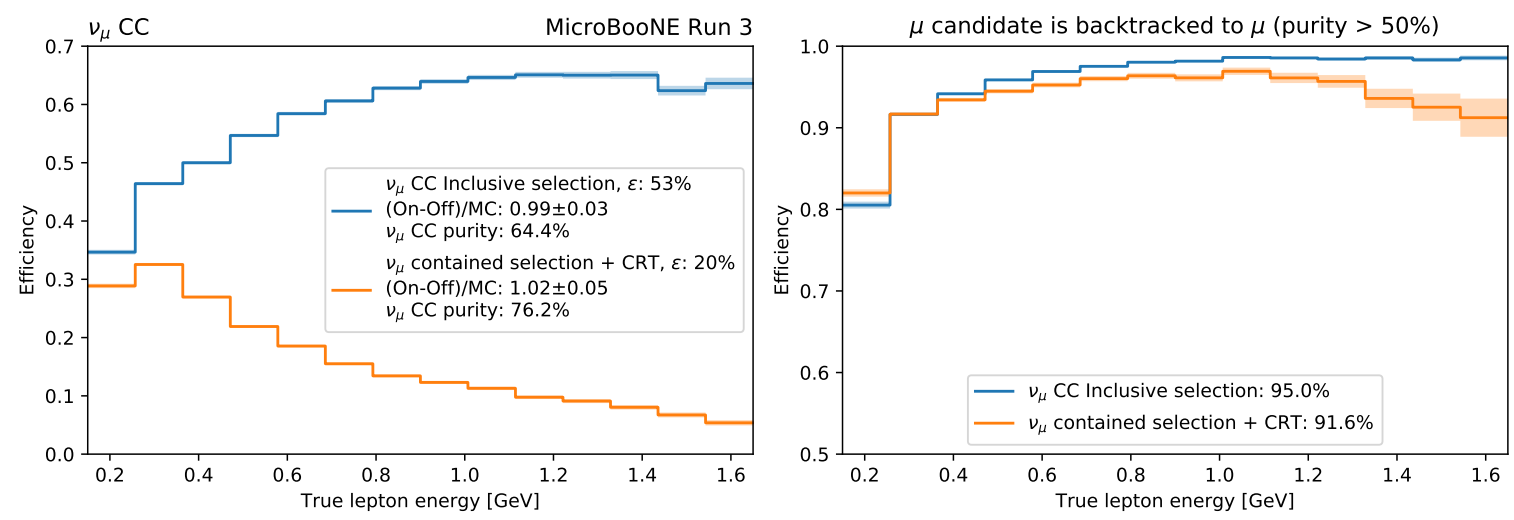

Figure 7.11: (Left) Efficiency of both $v_{\mu} \mathrm{CC}$ selections, as a function of the simulated muon energy. Summary of integrated data/MC ratio, purity and efficiency of the selection in the legend. (Right) Performance of the muon tagging for both the inclusive and the contained selections. The range of the horizontal axis $0.15 \mathrm{GeV}$ to $1.65 \mathrm{GeV}$.

stop is given by:

$$
R=\int_{E_{k i n, \mu}}^{0} \frac{\mathrm{d} E}{\mathrm{~d} x}(E) \mathrm{d} E
$$

This strictly monotone relation can be inverted to obtain the kinetic energy from the track length. The performance of this method relies on the containment and track length resolution of the particle's trajectory. The resolution of this method is shown in the right panels of Figure 7.13.

- MCS-based momentum: multiple Coulomb scattering was introduced in Section 6.2.2, and more specifically in Equation (6.2). The technique enables the measurement of the muon momentum by the scattering of the trajectory along track segments [87]. For this work, track segments of $14 \mathrm{~cm}$ are used. The method has a benefit that it can be used for uncontained tracks [88]. For optimal results, the track length should be at least $\mathcal{O}(1 \mathrm{~m})$. Furthermore, for tracks above approximately $2 \mathrm{GeV}$, the scattering angles become two small to be resolved from a variety of detector effects, such as space charge effect (Appendix B). The resolution of this method is shown in the left panels of Figure 7.13.

Figure 7.12 compares the MCS- and range-based momentum estimations of the muon candidate in the contained charged-current muon selection. It can be seen that the shapes of 
the data distribution - measured with both techniques independently - is slightly moved towards higher momenta. However, the $p$-values obtained from the two-sample KolmogorovSmirnov test (KS-test) - a metric to quantify shape disagreement - are not significant. Note that the data-set used corresponds to less than $1 \%$ of the total collected MicroBooNE data. In the near-future, larger statistics and systematic uncertainties evaluation will enable MicroBooNE to further investigate the discrepancy.
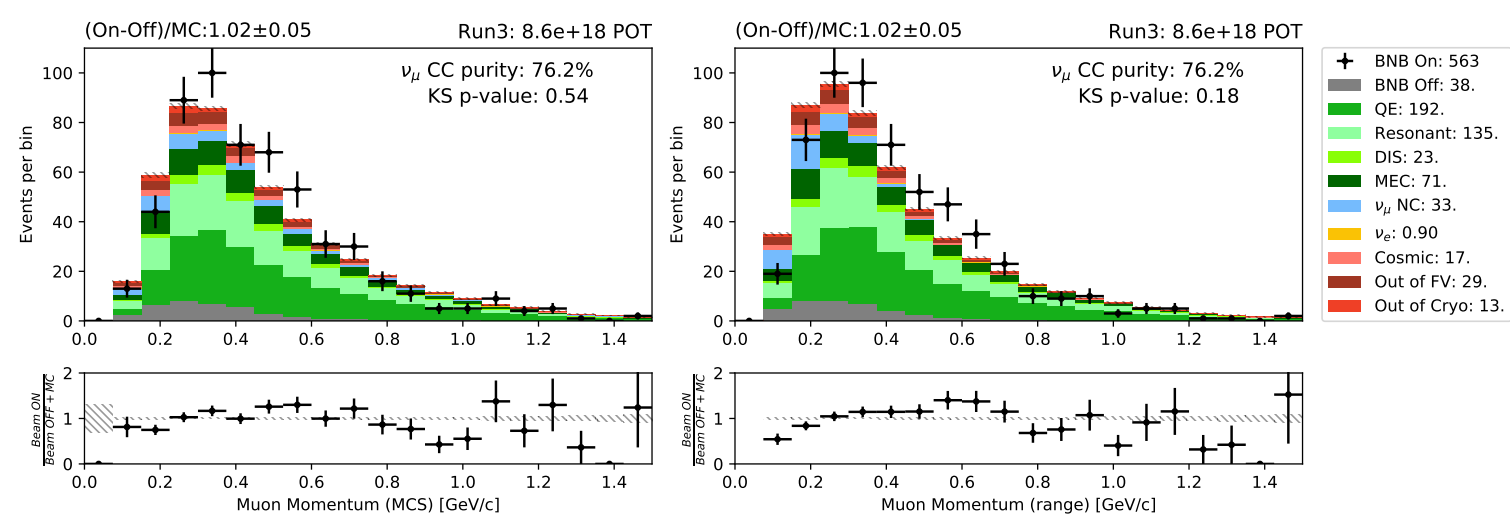

Figure 7.12: Comparison of MCS- and range-based momentum of the muon candidate in the contained charged-current muon selection. The $v_{\mu}$ CC signal events are sub divided by interaction mode. The reconstructed track length cut of $10 \mathrm{~cm}$ leads to an effective cutoff $\approx 0.1 \mathrm{GeV}$, as motivated in Figure 7.7 .

The advantage of the range-based momentum estimate in the contained selection is apparent in Figure 7.13. It can be seen that the range-based momentum both has a smaller bias less than $1 \%$ - and a superior resolution ${ }^{2}$. This selection will therefore be used to constrain the electron neutrino measurement in Chapter 9.

To conclude this section, it is instructive to look at the correlations between different muon variables used in this chapter. The Pearson correlation coefficient is a measure to inform us about the correlations between the variables used to select $v_{\mu}$ CC events and observables that one wants to measure. The coefficient takes values between +1 and -1 and is defined as:

$$
r_{x y}=\frac{\sum_{i=1}^{n}\left(x_{i}-\bar{x}\right)\left(y_{i}-\bar{y}\right)}{\sqrt{\sum_{i=1}^{n}\left(x_{i}-\bar{x}\right)^{2}} \sqrt{\sum_{i=1}^{n}\left(y_{i}-\bar{y}\right)^{2}}},
$$

\footnotetext{
${ }^{2}$ See Section 5.2.3 for a detailed description about how resolution is defined in this thesis.
} 

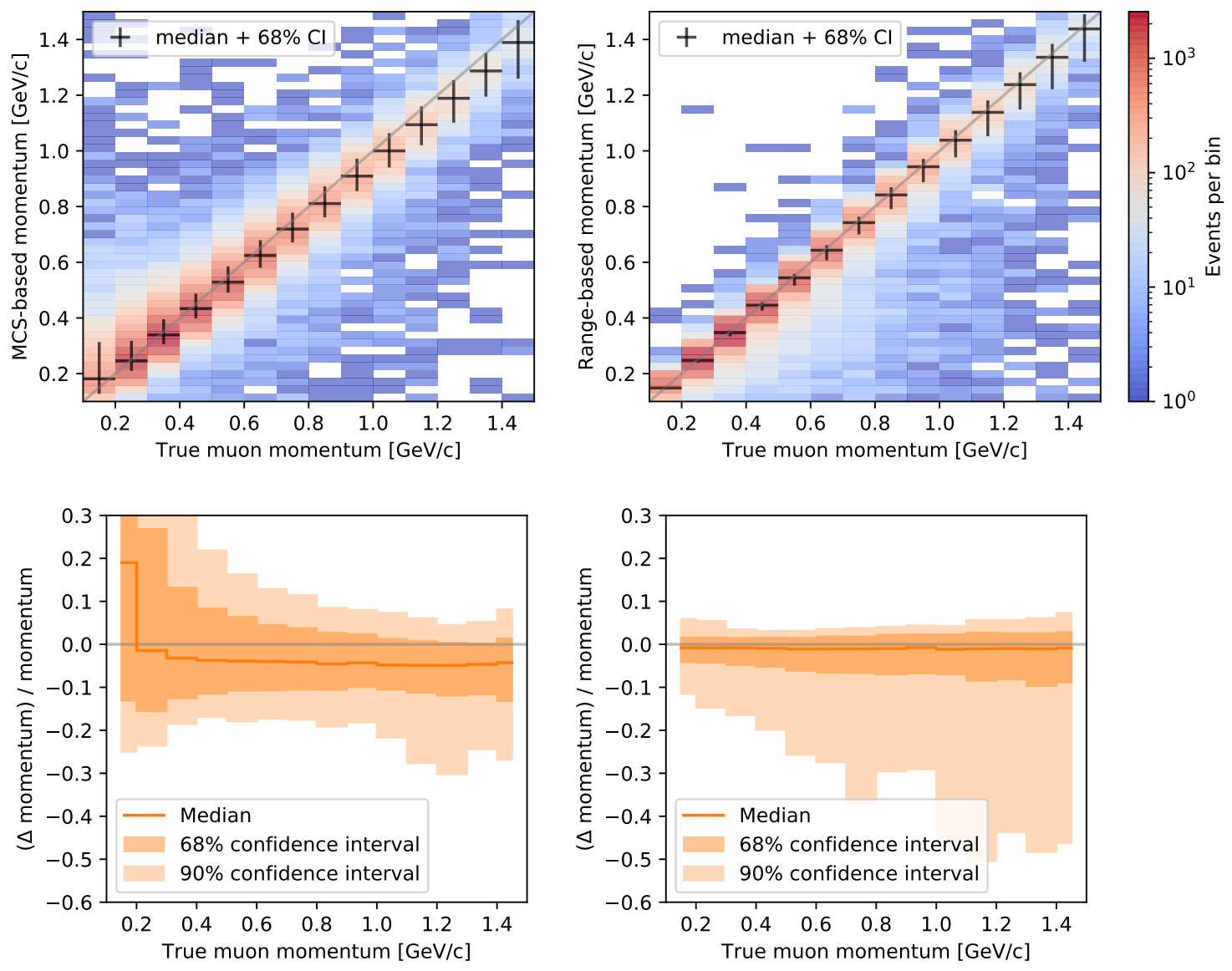

Figure 7.13: Resolution of the muon energy from the MCS-based (left panels) and the range-based (right panels) methods. (Top panels) The colour scale for the $2 D$ histograms is logarithmic. In black, the median and $68 \%$ confidence interval are given. (Bottom panels) The fractional bias, $68.3 \%(1 \sigma)$ and $90 \%$ confidence intervals, binned in the true muon momentum.

where $n$ is the sample size and $\bar{x}=\frac{1}{n} \sum_{i=1}^{n} x_{i}$, the sample mean; and analogously for $\bar{y}$ [92].

The correlation matrix is filled for all $v_{\mu} \mathrm{CC}$ events in the contained selection and is given in Figure 7.14.

A few observations can be made:

- The track length, track score and track PID likelihood are positively correlated. Since the matrix is calculated for selected muon tracks, this re-iterates that we are selecting long, track-like objects with a high muon PID likelihood.

- The range-based momentum and the reconstructed track length are fully correlated 
variables, which stems from the definition.

- The range-based momentum and the true momentum have a higher correlation than the MCS-based momentum and the true momentum. This supports the observations in Figure 7.13, that the range-based method leads to a lower bias and a better accuracy.

- The muon momentum is anti-correlated with the outgoing muon angle w.r.t. the incoming neutrino, $\theta$ (see Figure 3.6). This is expected to be the case for two reasons. First, higher energy neutrino-argon scattering events will be more boosted in the forward direction, and have a smaller $\theta$. Second, higher energy muon will travel a longer distance before stopping. Geometrically, because of the containment requirement, this is more likely to happen in the forward direction.

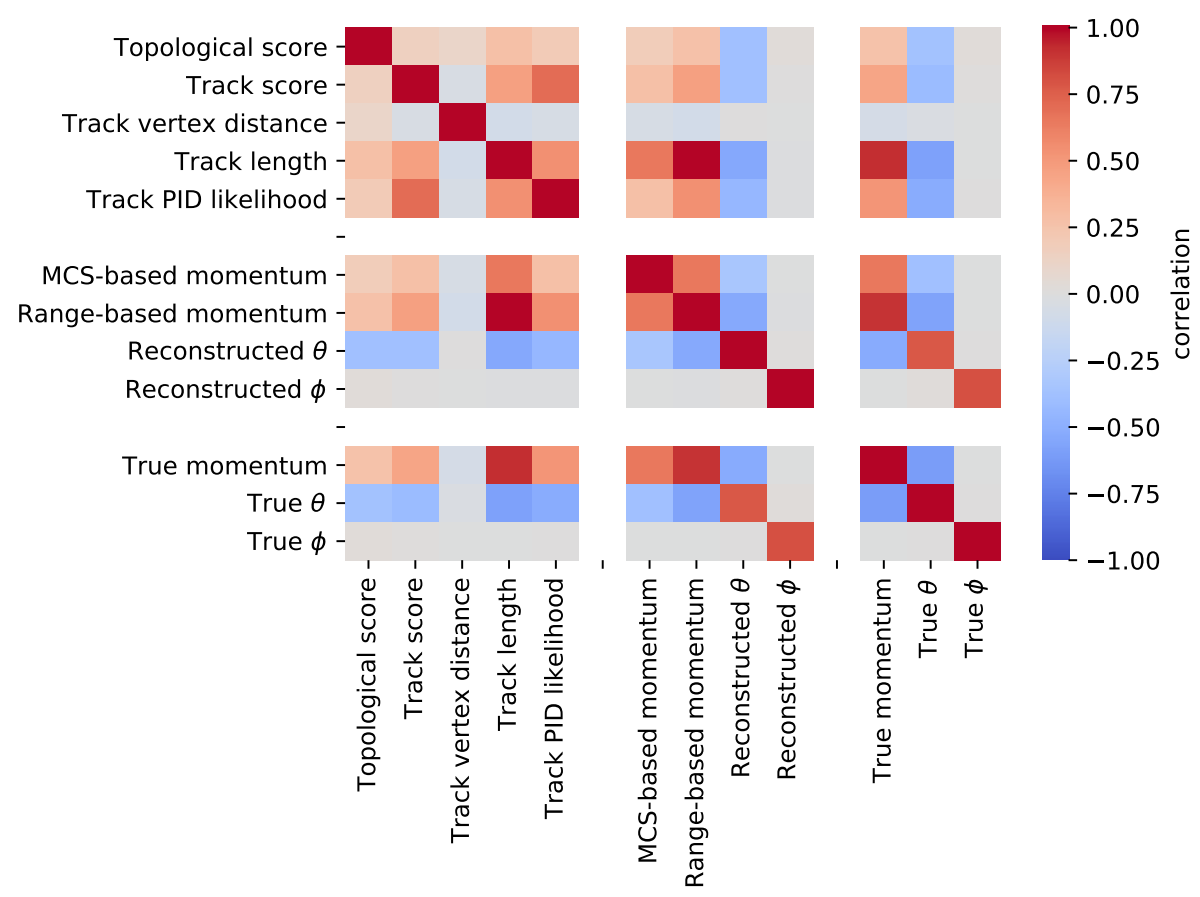

Figure 7.14: Correlation matrix of the variables of interest in the $v_{\mu} \mathrm{CC}$ analysis. The first group (topleft)) contains the variables used in the events selection and muon identification. The second group (middle) are the reconstructed lepton kinematics that can be compared with the backtracked counterparts in the third group (right-bottom). 


\subsubsection{Exclusive $v_{\mu} \mathrm{CC}$ selections}

The value of the inclusive $v_{\mu}$ CC selection, as foundation for exclusive final state topologies, is demonstrated in Figure 7.15. The exclusive selections here are obtained in two steps. First, events with shower-like objects and particles that are not near the reconstructed neutrino interaction vertex are discarded. Second, the tracks - apart of the muon identified track - are classified as proton or charged pion using the track log likelihood PID, which was introduced in Section 7.3.1. Three final state topologies are presented, each of them corresponds to an enriched contribution of an interaction mode described in Section 2.5:

- $v_{\mu}$ CC $0 \pi 1 \mathrm{p}$ : This 2-prong topology is predicted to be the dominant contribution of $v_{\mu} \mathrm{CC}$ interactions at the BNB neutrino energies. The most likely underlying process leading to this topology is QE, as illustrated in Figure 2.10.

- $v_{\mu}$ CC $0 \pi 2 \mathrm{p}$ : The two outgoing protons are an example of a $2 \mathrm{p}-2 \mathrm{~h}$ process. This topology is the preferred topology to isolate MEC interactions, illustrated in Figure 2.11. The contribution of meson exchange current events to the total cross section carries a large uncertainty in liquid argon experiments, making this channel very valuable.

- $v_{\mu}$ CC $1 \pi^{ \pm} 1 \mathrm{p}$ : The combination of an outgoing proton and an outgoing pion is characteristic for the decay of a $\Delta$ resonance (see Figure 2.12). Therefore, this channel is dominated by RES interactions.

Further studies of exclusive channels with high statistics and full systematic uncertainties will be crucial to improve the cross-section modelling, and therefore the energy reconstruction, for the next generation of LArTPC experiments. 

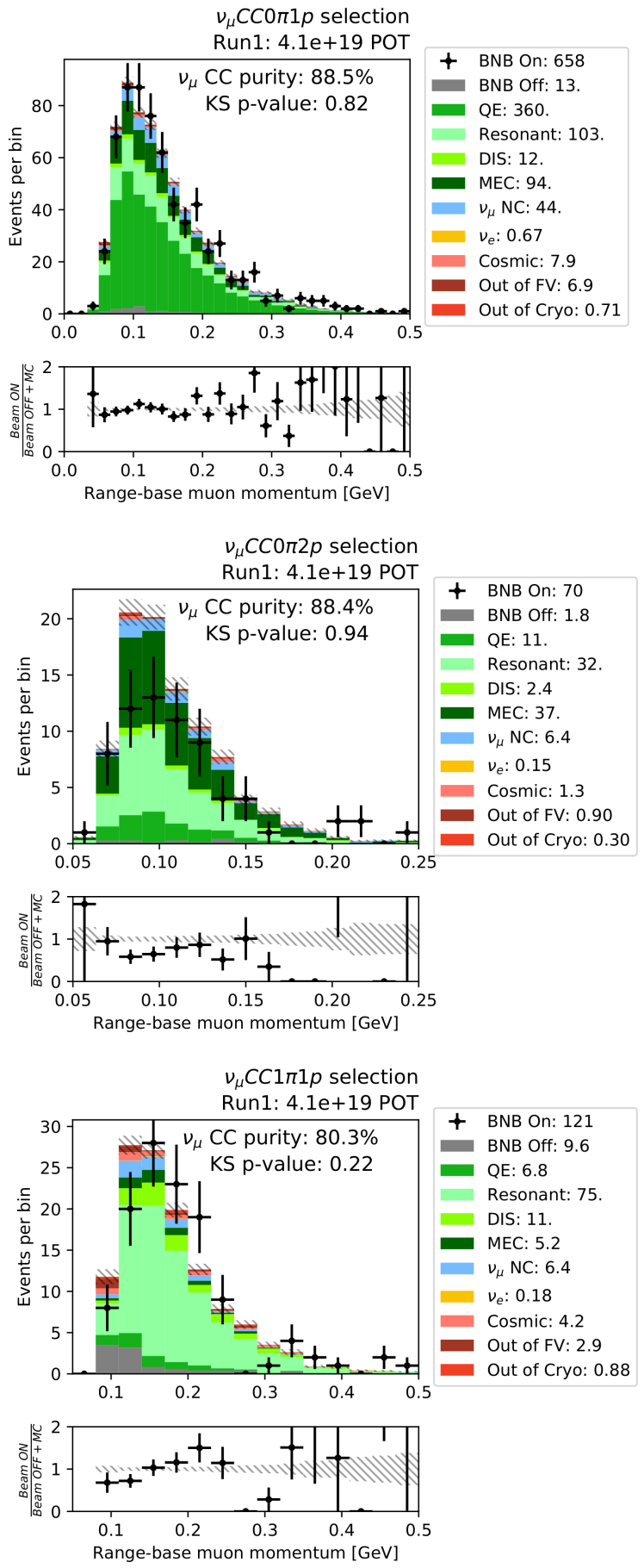

Figure 7.15: Demonstration of three exclusive channels based on the inclusive $v_{\mu}$ CC event selection. Each of the investigated topologies corresponds to a specific interaction mode: $v_{\mu} \mathrm{CC}$ $0 \pi 1 \mathrm{p}$ (top), $v_{\mu}$ CC $0 \pi 2 \mathrm{p}$ (middle), $1 \pi^{ \pm} 1 \mathrm{p}$ (bottom). The horizontal axis is the rangebased momentum estimation for the muon tagged track in the events. The legend for each panels indicates the different interaction mode of the selected $v_{\mu} \mathrm{CC}$ events. 


\section{Charged-Current Electron Neutrino Selection}

MicroBooNE is located in a muon beam and able to look for both the intrinsic $v_{e}$ CC contamination of the beam as well as potential new physics manifesting itself through $v_{\mu} \rightarrow v_{e}$ oscillation at $L / E \approx 2 \mathrm{~km} \mathrm{GeV}^{-1}$. Both rely on a selection of $v_{e} \mathrm{CC}$ events. Section 8.1 introduces the motivations and the strategy of the selection. The aim of the inclusive chargedcurrent electron neutrino selection is to identify electron neutrinos independently of their interaction mode or final state, and cover the full kinematic phase space of the outgoing electron. The ground work of the selection was described in Chapter 6 and the $v_{e}$ CC specific components will be described in Sections 8.2 to 8.5. The performance of the selection together with final distributions are discussed in Section 8.5.1. The evaluation of systematic uncertainties and the $v_{\mu}$ CC constraint will be the topic of Chapter 9. 


\subsection{Motivation}

\subsubsection{Electron neutrino selection philosophy}

Several proposals have been made to explain the nature of the MiniBooNE LEE anomaly (see Sections 2.4.1 and 3.3). A large amount of uncertainty remains in the community regarding what may have generated such an excess of electromagnetic events. This work investigates the $v_{e}$ appearance hypothesis. To best explore the potential new physics in the $v_{e}$ channel, an inclusive measurement of $v_{e} \mathrm{CC}$ interactions is performed. Above all, this analysis aims to measure and characterise the intrinsic $v_{e}$ contribution of $\approx 0.5 \%$ in the BNB (Table 3.1 and Figure 3.3). The understanding of the intrinsic electron neutrino content in MicroBooNE is a crucial step before any interpretation of a potential anomaly can be made. Additionally, the selection is constructed to minimise the reliance on lepton kinematic variables which depend upon neutrino-interaction models.

Analogously as was done for $v_{\mu}$ CC events in Section 7.2, the $v_{e}$ CC events are categorised by interaction modes or final state topologies. The four interaction modes of interest are Quasi-Elastic scattering, Meson Exchange Current, Resonant production and Deep Inelastic Scattering, as introduced in Section 2.5. Their relative contributions are shown in the right panel of Figure 3.16 and the left panel of Figure 8.1. The $v_{e}$ CC signal events as defined in Section 6.1 can be split into three final state topologies:

$-0 \pi 0 p$ : No protons or pions in the final state above $40 \mathrm{MeV}$ kinetic energy. The signature is a single electron shower.

- $0 \pi \mathrm{N} p$ : At least one proton $-\mathrm{N}>0$ - with $E_{k i n}(p)>40 \mathrm{MeV}$ in the final state but no pions above threshold.

- $\mathrm{M} \pi \mathrm{N} p$ : At least one pion $-\mathrm{M}>0$ - with $E_{k i n}(\pi)>40 \mathrm{MeV}$, no restrictions on the number of protons. Also referred to as $v_{e} \mathrm{CC}$ other.

Their contribution as a function of energy is given in the right panel of Figure 8.1. In the energy range of the $\mathrm{BNB}$, the dominant interaction mode is $\mathrm{QE}$, and the dominant final state is $v_{e}$ CC $0 \pi \mathrm{N} p$. 

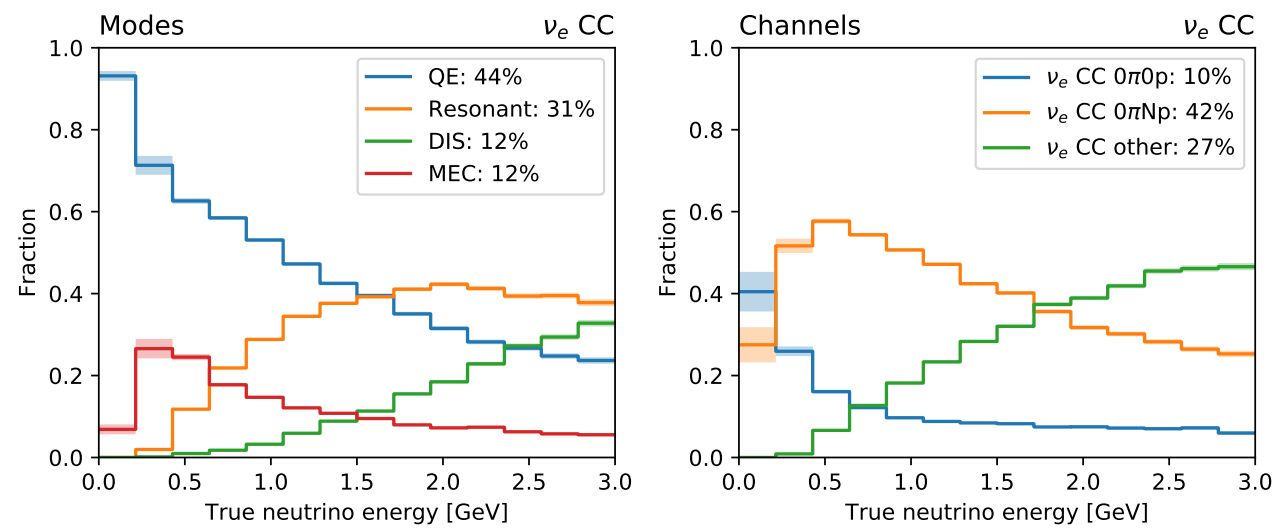

Figure 8.1: Breakdown of $v_{e} \mathrm{CC}$ events in interaction modes (left) and final state topologies (right) as a function of neutrino energy. Note that these are relative fractions and have to be multiplied by the BNB flux to retrieve the interaction rates. The relative contributions to the predicted $v_{e} \mathrm{CC}$ interaction rates from the $\mathrm{BNB}$ is given in the legend.

The selection strategy allows exploration of the kinematics of $v_{e}$ candidate events after their selection, for a full investigation of the origin of a potential anomaly. Implementing this choice requires the ability to fully leverage the information provided by the LArTPC for $v_{\mu}-v_{e}$ and $e-\gamma$ separation. The tools for this goal will be described in subsequent sections.

\subsubsection{Selection strategy}

As previously mentioned, of all MicroBooNE recorded events triggered by the BNB, only one in approximately 600 contains a neutrino interaction in the TPC. Furthermore, every recorded event, with or without neutrino interaction, contains approximately 15 cosmicmuon interactions. Out of all the predicted neutrino interactions - NC and CC, $v_{\mu}$ and $v_{e}-$ in the TPC, only $0.48 \%$ is a $v_{e}$ CC event. The selection has therefore three goals:

- Reject cosmic-induced activity.

- Identify the final state electron and reject events with muons in the final state.

- Distinguish electrons from photons. This is essential to reduce the large fraction of NC $\pi^{0}$ events where the $\pi^{0}$ decays into two photons, and it is crucial to identify the origin of a potential anomaly. 
The flowchart in Figure 8.2 introduces the selection strategy followed in this chapter. Cosmic rejection is obtained by combining the tools introduced in Chapter 6 (the NeutrinoID) with additional cuts to further reject cosmic activity. At the preselection stage, an electron candidate shower is identified. This shower enters the electron PID stage. All other reconstructed objects in the event, both track/shower-like are evaluated independently in the other daughters step. The characterisation of all reconstructed objects is now combined in the final $v_{e} \mathrm{CC}$ event selection.
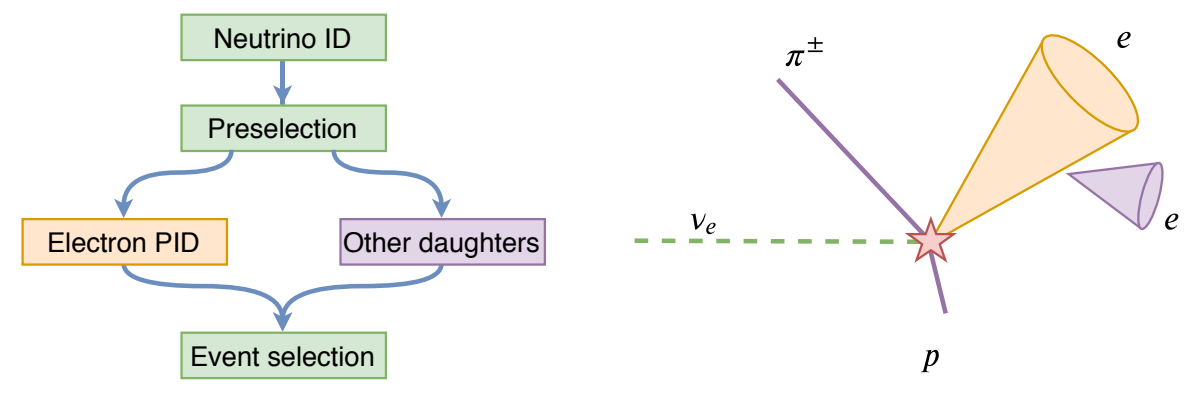

Figure 8.2: (Left) Flowchart indicating the different steps in the $v_{e}$ CC selection. (Right) A hypothetical $v_{e} \mathrm{CC}$ event with multiple reconstructed showers and tracks. At the preselection stage, an electron candidate shower is identified (orange) and electron particle identification is performed on this shower. The other reconstructed objects (purple) are classified to improve background rejection before merging the outputs and perform the final event selection.

\subsubsection{MiniBooNE LEE signal model}

Many models can be devised to explain the MiniBooNE LEE. They can, grosso-modo, be categorised as photon-like and electron-like interpretations of the excess. One photon-like explanation predicts an increased NC resonant $\Delta$ production, with subsequent radiative decay, illustrated in Figure 8.3 and the right panel of Figure 8.4. The photon-like hypothesis is investigated in [93].

This work focuses on the electron-like hypothesis and this section describes the signal model chosen by the MicroBooNE collaboration. It is important to stress that any signal model carries a set of important assumptions and caveats, and that the ultimate goal of the analysis is to measure the rate of $v_{e} \mathrm{CC}$ interactions in the $\mathrm{BNB}$, reporting whether the 

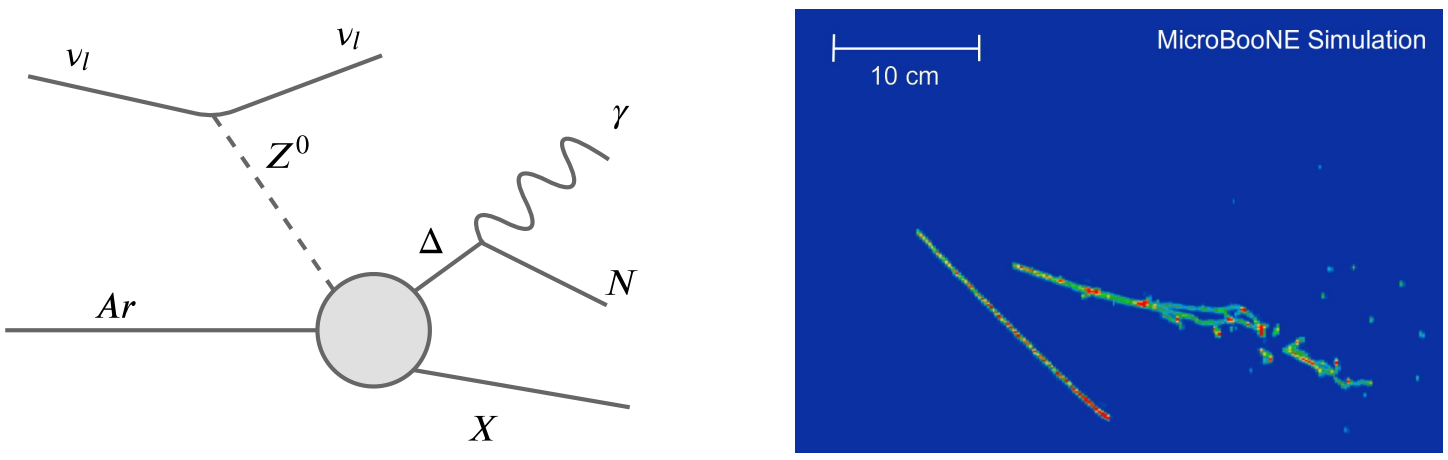

Figure 8.3: Example of a NC $\Delta \rightarrow N \gamma$ decay. (Left) The Feynman-like diagram of the process. (Right) A simulated event of this kind in MicroBooNE where the radiated shower and the $X$ particle - in this case a proton - are visible.

observation is consistent or not with the predicted $v_{e}$ content in the beam.

The signal model used to generate simulated events in MicroBooNE is the MiniBooNEunfolded LEE model [94], referred to as $v_{e}$ LEE in the figures. In this model, all excess LEE events are assumed to be due to $v_{e}$ interactions with a true energy obtained by unfolding from the reconstructed CCQE energy of the MiniBooNE LEE events. This procedure is performed by relying on MiniBooNE's energy smearing matrix. The resulting true neutrino energy distribution is shown in the left panel of Figure 8.4. One limitation of this model, outlined in Section 2.4.1 is that the actual energy of the neutrinos might be underestimated due to the CCQE-like energy calculation approximation (Equation (2.22)). The unfolded model is composed of a binned event distribution of the expected $v_{e}$ spectrum and situated from $200 \mathrm{MeV}$ to $600 \mathrm{MeV}$.

It is especially important to note that the chosen model strongly favours the interpretation of MiniBooNE events as originating from very low neutrino energies. Instead of tailoring the selection to this specific corner of the phase space, it was decided to develop an inclusive and kinematically-unbiased analysis. In addition to the MiniBooNE-unfolded LEE model, a $3+1$ sterile-neutrino oscillation model is explored in Section 9.3.3. 

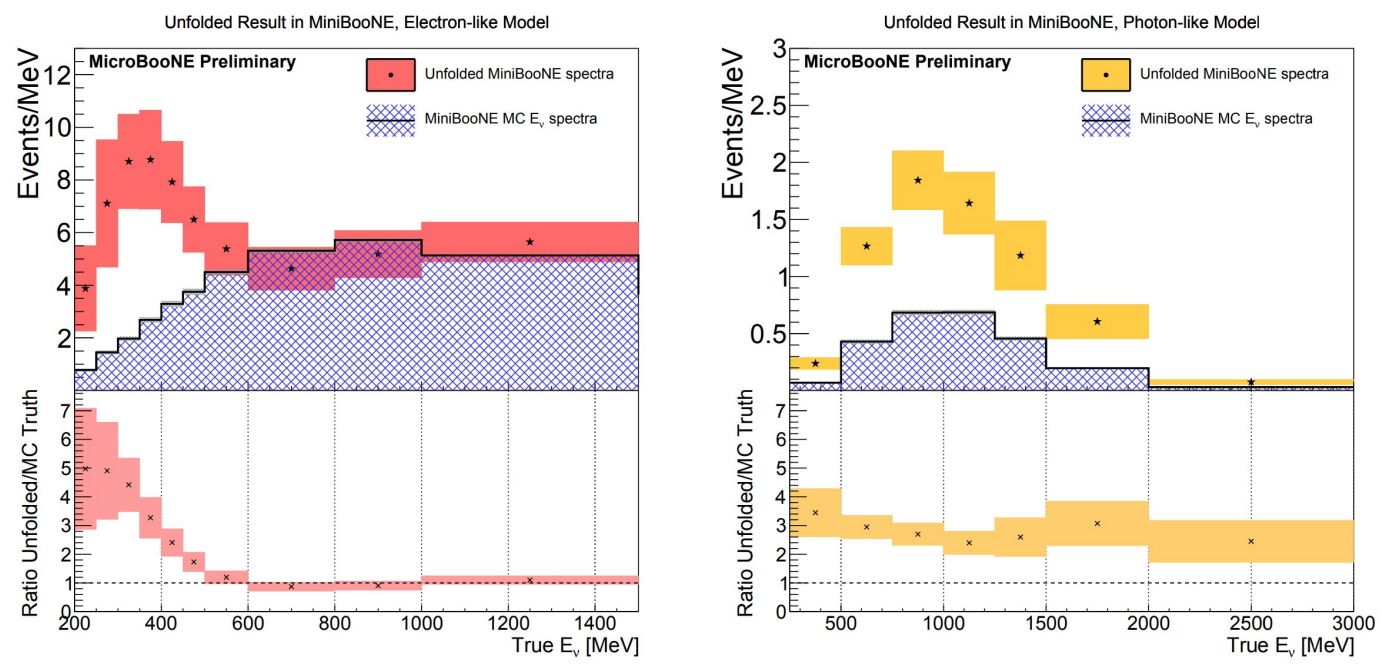

Figure 8.4: Results of unfolding the MiniBooNE LEE under both the electron-like (left) and photonlike (right) hypotheses. Both are obtained using the D'Agostini iterative unfolding algorithm [95]. The unfolded spectra itself, as well as the MiniBooNE Monte Carlo spectrum are plotted, indicating the energy dependent increase necessary to account for the observed LEE, highlighted by the ratio of these which is shown in the bottom panels. Figure from [94]. 


\section{2 $v_{e}$ CC preselection}

The preselection aims, at first, to reject the backgrounds arising from cosmic activity; second, to identify a plausible electron candidate. The figures in this chapter will follow a largely similar categorisation of events as was used in Chapter 7 with a few differences:

- The $v_{e}$ CC events are subdivided into the three categories introduced in Section 8.1, based on their topology: $v_{e} \mathrm{CC} 0 \pi 0 \mathrm{p}, v_{e} \mathrm{CC} 0 \pi \mathrm{Np}(\mathrm{N}>0)$ and $v_{e} \mathrm{CC} \mathrm{M} \pi \mathrm{Np}(\mathrm{M}>0)$.

- The $v_{e}$ CC events are separated into events containing a $\pi^{0}$ in the final state and the ones without a neutral pion.

- The neutral-current neutrino interactions are divided depending on the presence of final state neutral pion.

- The MiniBooNE-unfolded LEE hypothesis is added on top of the MC prediction.

The importance of separating events with final state neutral pions stems from the fact that the $\pi^{0} \rightarrow \gamma \gamma$ can mimic an electron shower if one of the two photons is not reconstructed or the two photons are co-linear. This will further be discussed in the next section.
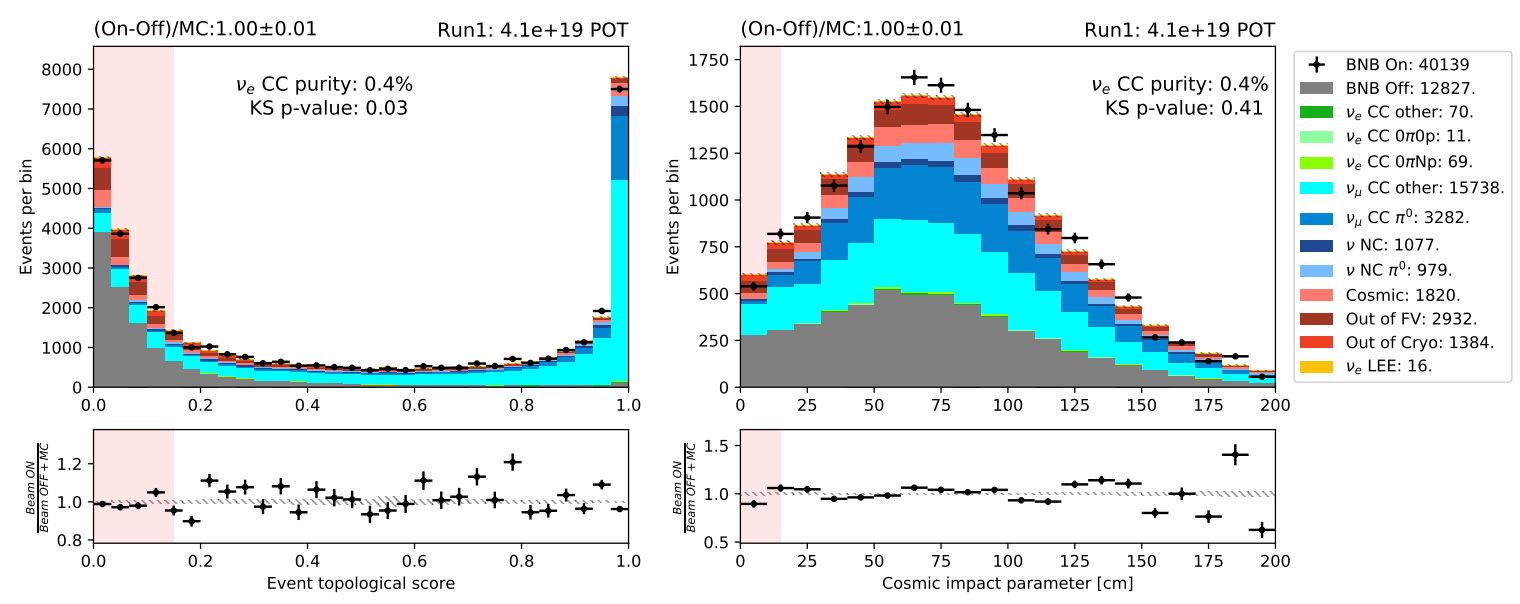

Figure 8.5: The event topological score as described in Section 6.3.1 (left) and the cosmic impact distance as described in Section 7.2 (right). Events in the shaded red region are rejected. At this stage, the signal $v_{e}$ CC events (green) only make up $0.4 \%$ and are not visible. 
The first step in the preselection follows the philosophy of the $v_{\mu} \mathrm{CC}$ selection. The reconstructed vertex is required to be inside the fiducial volume (Figure 6.1). Subsequently, a cut is placed on the topological score and the cosmic impact distance. Figure 8.5 illustrates both cuts, but the $v_{e}$ CC purity of $0.4 \%$ is too low to clearly see the signal events. The topological cut rejects $18 \%$ of signal events, mostly badly reconstructed events at low energy. Despite the significant loss in signal, the effectiveness to remove most Beam Off background ( $84 \%)$ makes it a valuable trade-off. The cosmic impact distance cut removes less than $1.5 \%$ of $v_{e}$ CC events while rejecting $3 \%$ of cosmic background events. On more recent data, run period three and later, the CRT system is used to gain additional Beam Off rejection (see Section 6.5).
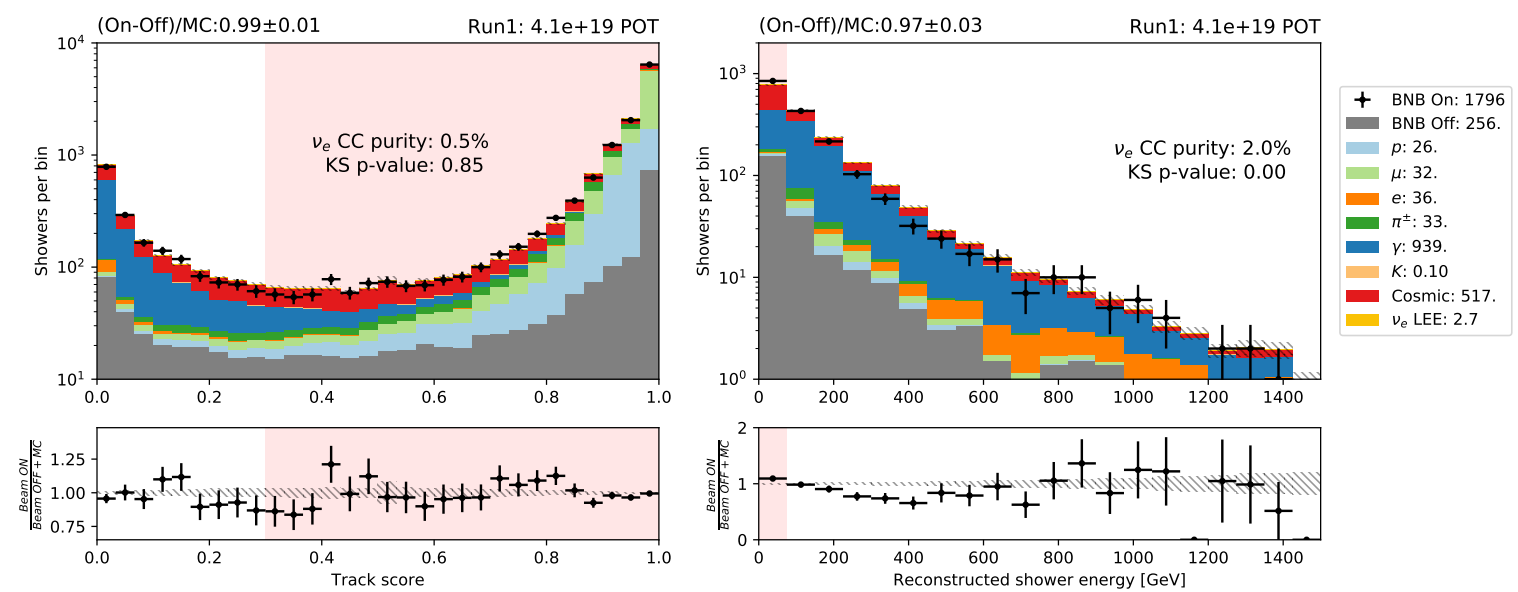

Figure 8.6: (Left) The track score as described in Section 4.2.3 The histogram is filled for all objects passing the reconstruction-based quality requirements (see text for details). Objects with a low track score are more shower-like. (Right) The reconstructed shower energy obtained from the deposited charge on the collection plane. The histogram is filled with all showers passing the topological score cut and previous requirements. The bin size on the horizontal axis is $75 \mathrm{MeV}$. Events in the shaded red region are rejected. Both cuts aim to select electron showers. Note the logarithmic scale on the vertical axis in both panels.

Second, an electron candidate is identified. From inside the collection of daughters, showerlike objects are selected using a cut on the track score of the reconstructed object, illustrated in the left panel of Figure 8.6. From the $v_{e}$ CC events passing the previously described cuts, $81 \%$ fulfil this requirement by having a plausible candidate shower. At the lowest 
electron energies $-\lesssim 300 \mathrm{MeV}$ - the electromagnetic showers start to look more track-like, leading to higher track-scores and causing a decrease in selection efficiency at this stage. Finally, to reject Michel electrons produced in the decays of cosmic-induced muons, peaking at $m_{\mu} / 2=53 \mathrm{MeV}$, at cut on the reconstructed shower energy is placed at $75 \mathrm{MeV}$. The Michel-rejection cut is illustrated in the right panel of Figure 8.6 and leads to a rejection of $65 \%$ of the remaining cosmic background events and a $7 \%$ loss in signal events. In the unlikely case that there are multiple showers qualifying as plausible electron candidate after these cuts, the highest energy one is selected as the electron candidate.

Table 8.1: Preselection requirements for the $v_{e} \mathrm{CC}$ inclusive.

\begin{tabular}{|c|c|}
\hline Cut goal & Cut definition \\
\hline \hline & Selected by the NeutrinoID \\
Cosmic rejection & $\begin{array}{c}\text { Reconstructed neutrino vertex is in FV } \\
\text { Topological score }>0.15 \\
\\
\text { Cosmic impact distance }>15 \mathrm{~cm} \\
\text { If available, CRT tools }\end{array}$ \\
\hline Signal topology & Electron shower candidate \\
\hline Michel rejection & Minimum $75 \mathrm{MeV}$ shower energy \\
\hline
\end{tabular}

The preselection requirements are summarised in Table 8.1. The efficiency of the preselection is $(53.8 \pm 3.0) \%$. Combined with the efficiency of the NeutrinoID of $83.3 \%$, this leads to a $v_{e}$ CC event selection with an energy-integrated efficiency of $44.8 \%$. This is equivalent to $\mathcal{O}(700)$ events per $10.1 \times 10^{20}$ POT, the total collected MicroBooNE data-set between 2015 and 2019 (see Figure 10.1). The number of simulated LEE signal events passing is $\mathcal{O}(50)$ per $10.1 \times 10^{20}$ POT. The purity at this stage is $3.0 \%$.

After the preselection cuts, the $v_{e}$ CC purity is still low (3.0\%). Figure 8.7 shows the angular distributions of the electron candidate shower highlighting the dominant background categories: the main background are photons originating from $\pi^{0}$ in the final state. The next section will specifically discuss tools to achieve $e-\gamma$ separation. 

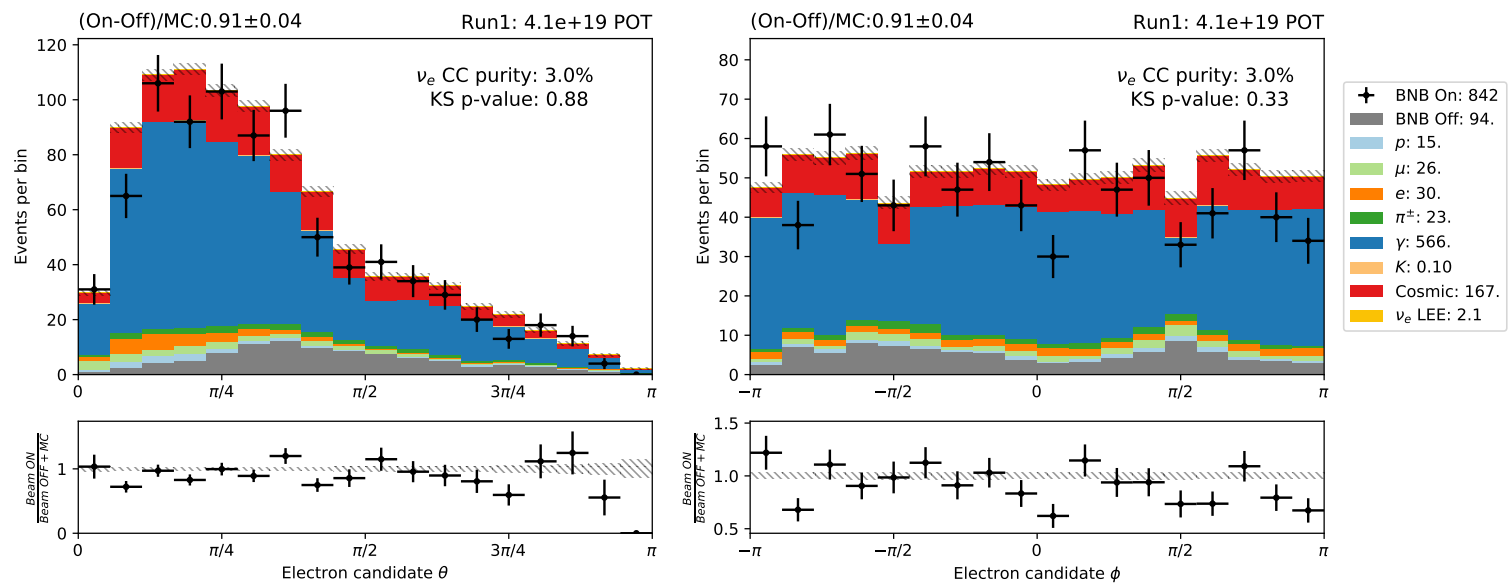

Figure 8.7: Electron shower candidate $\theta$ (left) and $\phi$ (right) after the preselection. The histogram is categorised using the reco-truth matched particle type corresponding to the electron candidate shower. 


\subsection{Electron Identification}

Distinguishing electron from photon electromagnetic showers is one of the crucial steps required to perform a measurement of $v_{e} \mathrm{CC}$ interactions. Photon backgrounds are largely caused by neutrino interactions with $\pi^{0} \rightarrow \gamma \gamma$ in the final state. This topology dominates the $v_{e}$ event rate by approximately an order of magnitude.

The electron identification in this analysis is performed by a gradient boosted decision tree. The variables used to maximise the electron-photon separation are listed in Section 8.3.2. The correlation of the variables and the outcome of the classification process is discussed in Section 8.3.4. The first section gives a general overview of electromagnetic showers in LAr.

\subsubsection{Electromagnetic shower formation}

Electromagnetic shower formation in liquid argon is relevant to both electrons and photons. For electrons, the dominant processes are:

- Ionisation and excitation: The energy loss per unit of distance is described by the BetheBloch function. For an electron, the relativistic rise is weak and it can be treated as a Minimal Ionising Particle (MIP), depositing approximately $2.1 \mathrm{MeV} \mathrm{cm}^{-1}$ in LAr.

- Bremsstrahlung: Bremsstrahlung is electromagnetic radiation produced by the deceleration of a charged particle after passing through the electric and magnetic fields of a nucleus. Above a critical energy $-35 \mathrm{MeV}$ in liquid argon, bremsstrahlung is the dominant process through which electrons lose energy.

For photons, two different interaction processes are important:

- Pair production: When the photons energy exceeds the threshold of two times the electron mass, it can produce an $e^{+} e^{-}$pair. This becomes the dominant process above $\approx 10 \mathrm{MeV}$.

- Compton scattering: A photon can scatter of an atomic electron in argon, thereby liberating the electron while deflecting its own trajectory. The relative cross sections of both photon processes are given in Figure 8.8. 


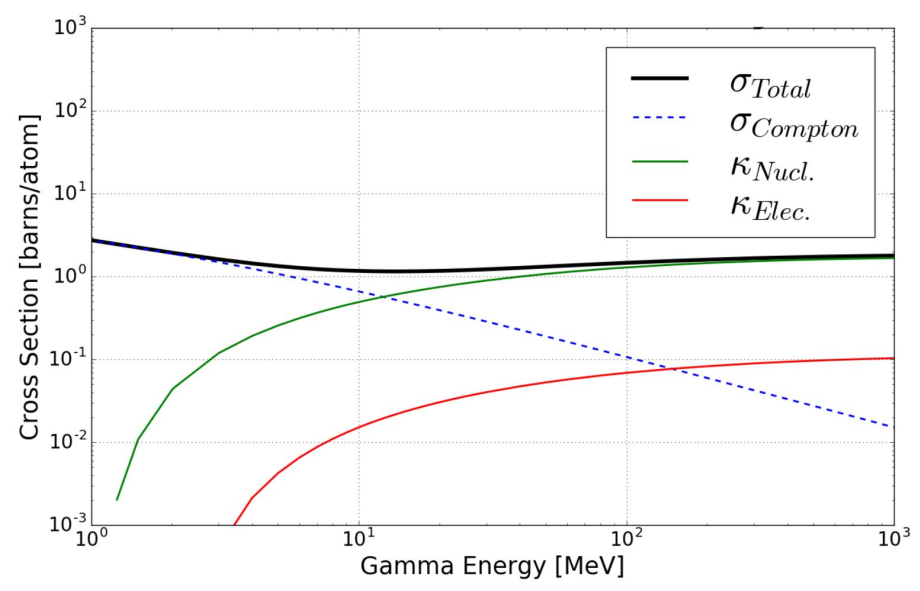

Figure 8.8: Cross section of gammas on argon between $1 \mathrm{MeV}$ and $1 \mathrm{GeV}$, the relevant energies in MicroBooNE. Here, $\kappa$ refers to the pair production cross section for the nuclear field and electron field. Compton scattering is dominant below $10 \mathrm{MeV}$. Figure from [96].

The concept of the electromagnetic shower arises from the interplay between these four processes, leading to an avalanche of electrons and photons in the bulk of the shower, illustrated. In the left panel of Figure 8.9. In every step of the shower formation - through Bremsstrahlung, pair production and Compton scattering - the amount of particles doubles, effectively dividing the average energy per electron/photon by a factor two. For liquid argon, the conversion length $X_{0}$ for the different processes is roughly similar and equal to $\approx 15 \mathrm{~cm}[12]$.

$$
l=X_{0} \cdot t
$$

where $t$ is the number of steps in the shower formation. The total amount of particles at step $t$ is:

$$
N=2^{t}
$$

The shower formation comes to a halt when the electron energy falls below the Bremsstrahlung threshold energy, $E_{c}$ of $35 \mathrm{MeV}$ [12]:

$$
N_{\max }=\frac{E}{E_{c}}
$$



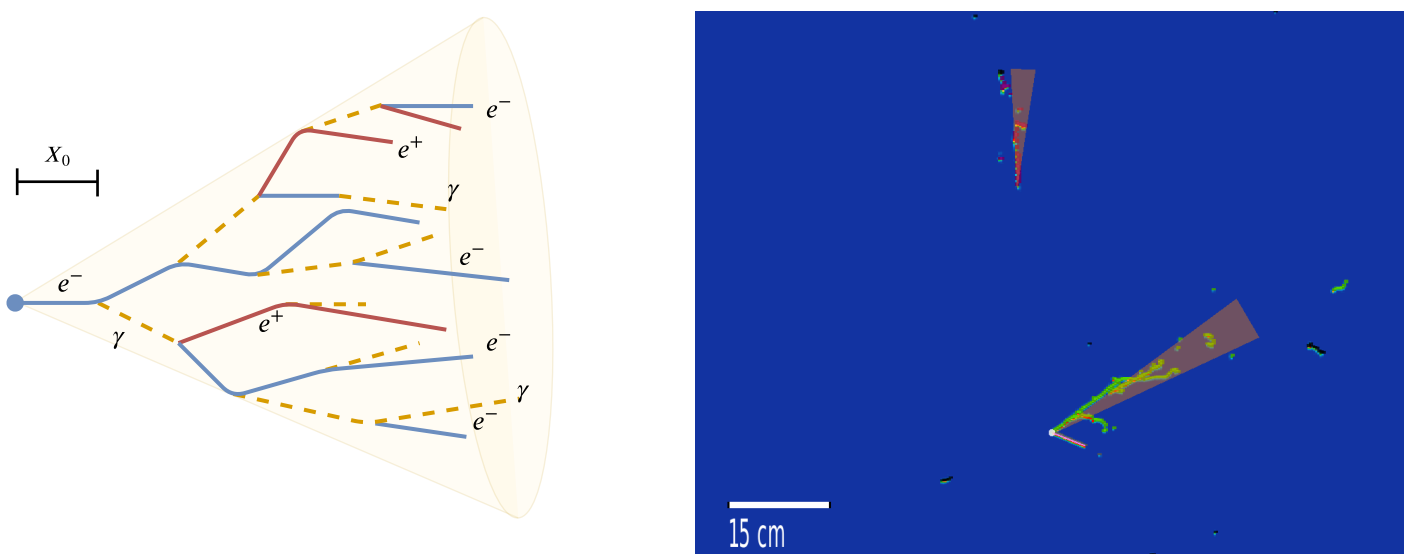

Figure 8.9: (Left) Illustration of shower formation initiated by an electron (blue). The continuous line of the positrons (red) and electrons represent ionisation losses while the dotted yellow line represents photons. The electrons interact through Bremsstrahlung, radiating a photon. The photons most often undergo pair production, but also Compton scattering, liberating an electron; both are included in the diagram. (Right) Simulated NC event with a proton and a $\pi^{0}$ in the final state. The $\pi^{0}$ immediately decays two photons, the conversion distance for photon attached to the proton is exceptionally short. The conversion distance for the detached photon is of the order of $50 \mathrm{~cm}$, exceptionally long. The red cones overlaid on the event indicate the reconstructed shower object.

Therefore, the length of the shower can be approximated by:

$$
l=X_{0} \cdot \frac{\log \left(E / E_{c}\right)}{\log (2)}
$$

For typical electron shower energies at MicroBooNE, the length of the electromagnetic shower is in the range $20 \mathrm{~cm}$ to $100 \mathrm{~cm}$. Due to their lower average energy, for photon showers from $\pi^{0}$ decays the length is a bit shorter.

The transverse development of the shower formation is dominated by Bremsstrahlung, Moliere theory predicts the radius of the cone to be independent of the energy and given by [97]:

$$
R_{m}=\frac{21.2 \mathrm{MeV}}{E_{c}} \cdot X_{0}
$$

For liquid argon, the diameter of the electromagnetic shower is therefore estimated to be $\mathcal{O}(20 \mathrm{~cm})$. This approximation holds for the bulk of the shower. In the tails, the contribution from low energy photons through Compton scattering becomes important, effectively 
widening the shower cone. It is important to note that these estimates - although they correspond well with observations in MicroBooNE- are affected by detector threshold and event reconstruction effects. More information about electromagnetic shower characterisation in liquid argon can be found in references $[98,99,75]$.

As can be deduced immediately from the antecedent discussion, the bulk of the shower is identical for both electrons and photons. Furthermore, the longitudinal shower development has a logarithmic dependence on the energy and the radius is approximately constant. Nonetheless, as can be seen from Figure 8.9, the start of the shower is dependent on the particle type.

Three key features distinguish events with $\pi^{0}$-induced photon showers from $v_{e}$ interactions:

- The presence of a second electromagnetic shower.

- The non-zero conversion distance (gap) separating the neutrino interaction vertex from the shower start point

- The increased calorimetric activity $(\mathrm{d} E / \mathrm{d} x)$ near the start of the shower due to the overlapping ionisation segments of $e^{+} / e^{-}$from the photon pair production.

Variables related to these features will be extracted from the reconstruction objects and described in the next section.

\subsubsection{Shower variables}

This section introduces the variables related to the electron shower identification and $\pi^{0}$ rejection. For every variable discussed here, the data/MC agreement is given at the end of the section in Figure 8.16.

\section{Vertex distance}

A photon-initiated shower will only start when the photon undergoes conversion through pair production (or less frequent, through Compton scattering). This effectively leads to a shower vertex which is displaced from other activity located near the neutrino interaction 
vertex. The conversion is an exponential process with a path length of $\mathcal{O}\left(X_{0}\right)$. Two example photon showers are shown in the right panel of Figure 8.9. The distance between the reconstructed start point of the shower and the reconstructed neutrino interaction vertex is therefore essential in $e-\gamma$ separation. This variable is especially powerful when additional vertex activity enables an accurate neutrino vertex reconstruction. For the channels without additional vertex activity, such as $v_{e} \mathrm{CC} 0 \pi 0 p$ events, the reconstructed vertex and shower start coincide.

\section{Energy loss at shower start}

An electron shower will start with a track-like trunk $-\mathcal{O}(10 \mathrm{~cm})$ - during which it will have a $\mathrm{d} E / \mathrm{d} x$ value of $\approx 2.1 \mathrm{MeV} \mathrm{cm}^{-1}$, before it will undergo Bremsstrahlung. The majority of photons manifest themselves in the TPC through the ionisation released by the $e^{+} / e^{-}$pair produced via pair-conversion. The electron-positron pair is highly aligned and overlaps on the mm-scale, leading to a doubly-ionising charge-segment compared to electron showers. Three different variables, listed below, characterise the $\mathrm{d} E / \mathrm{d} x$ at the start of the shower. All three of them are obtained using a track fit to the trunk of the shower, as was described in Section 4.2.3 and is illustrated in Figures 8.10a and 8.10b.

$-4 \mathrm{~cm}$ range, collection plane only. The $\mathrm{d} E / \mathrm{d} x$ value obtained from the first $4 \mathrm{~cm}$ of the shower start on the collection plane $(Y)$.

$-4 \mathrm{~cm}$ range, plane-averaged. The $\mathrm{d} E / \mathrm{d} x$ is obtained for each of the three planes - two induction planes and the collection plane - independently. The obtained values are then averaged, weighted by the number of reconstructed hits on the respective planes in the first $4 \mathrm{~cm}$.

$-1 \mathrm{~cm}$ to $5 \mathrm{~cm}$ range, collection plane only. Here, hits in the first centimetre of the shower trunk are omitted from the $\mathrm{d} E / \mathrm{d} x$ calculation and the subsequent $4 \mathrm{~cm}$ are used. This is motivated by cases where the first few hits of a shower merge activity from short protons near the interaction vertex, causing a large $\mathrm{d} E / \mathrm{d} x$ which hampers the ability to identify the shower as an electron. 


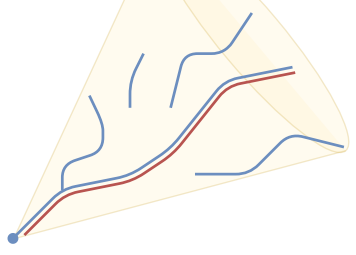

(a) Track fit to shower trunk

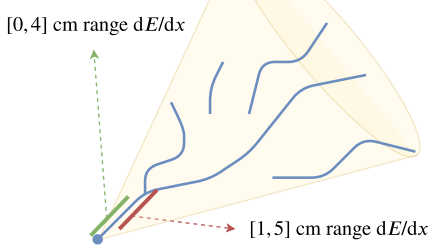

(b) $\mathrm{d} E / \mathrm{d} x$ at the start of the shower

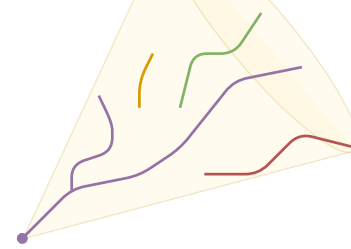

(c) Number of subclusters

Figure 8.10: Subset of variables used for electron identification.

\section{Geometrical variables}

In addition to the $\pi^{0}$-induced photon background, it can be seen from Figure 8.7 that there is still a non-negligible fraction of cosmic-induced backgrounds. These often corresponds to muons decaying into a Michel electron that are reconstructed as a shower object. Examples of such events are given in Figure 8.11. To improve on the track-shower separation performed by Pandora (Section 4.2.3), additional variables which leverage different aspects of shower topologies are devised:
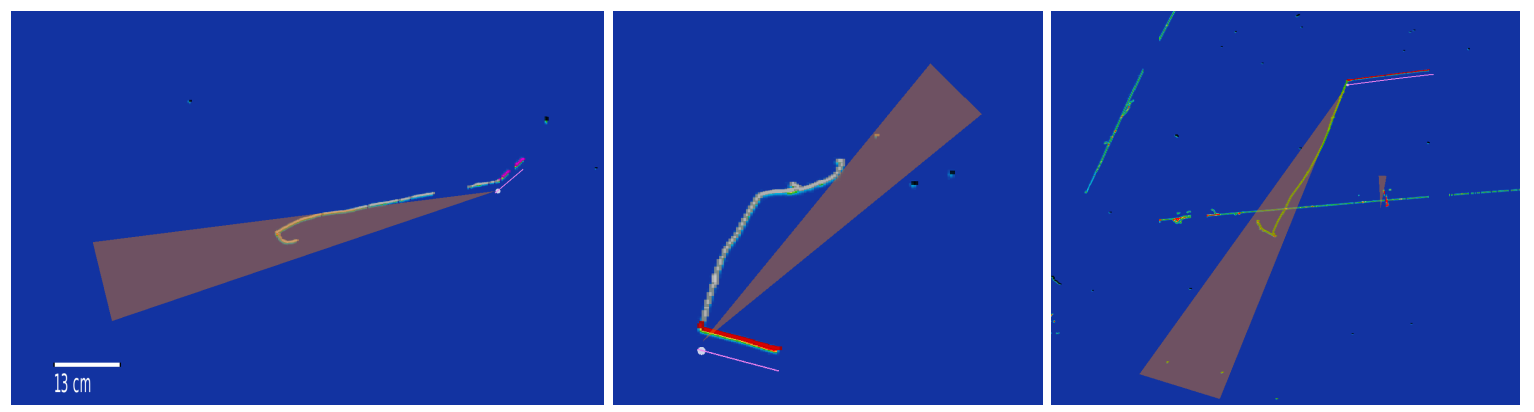

Figure 8.11: Examples of simulated events in which a muon decays into a Michel electron. The shown events were selected to illustrate the reconstruction of the muon and the electron as a single shower object.

- Shower subclusters. (see Figure 8.10c) Electromagnetic showers are often comprised of several branches isolated by gaps caused by photons propagating through the detector medium. This variable leverages this aspect by counting the number of isolated segments of charge associated to reconstructed showers. This quantity is a sum of 
such clusters from all three planes.

- Moliere angle. This quantity characterises the profile of reconstructed electromagnetic showers. It is computed using the $3 D$ charge deposits. For each $3 D$ point, the angle between the shower's direction and the point is calculated. The average of all such angles is defined as the Moliere angle.

\section{Second shower tagging}

Events with a final state $\pi^{0}$ that are reconstructed with a single electromagnetic shower in the final state occur because either the second photon escapes the active volume completely (irreducible) or because the second shower is not reconstructed. The dominant causes of this second case are:

- highly-boosted $\pi^{0}$ decays, in which two aligned photons are merged into one shower.

- photons which go undetected in the event reconstruction chain, often low in energy (below $\mathcal{O}(100 \mathrm{MeV})$ ).

To improve on our $\pi^{0}$ rejection, we try to identify a second shower candidate by looking for a set of clustered hits on the collection plane. For this cluster, the distance to the reconstructed neutrino vertex, along with the number of hits contained in it are used to identify $\pi^{0}$ events. The variables are illustrated in Figure 8.12. In the example event of Figure 8.12a, these quantities are computed for the circled black cluster of charge which represents a missed photon in the reconstruction.

\subsubsection{Classification using a boosted decision tree}

The library used in this thesis to perform the classification task is XGBoost [100]. XGBoost stands for "Extreme Gradient Boosting", where the term "Gradient Boosting" originates from the paper Greedy Function Approximation: A Gradient Boosting Machine, by Fried$\operatorname{man}[101]$.

The classifier is a supervised learning mechanism that performs a mapping between a set 


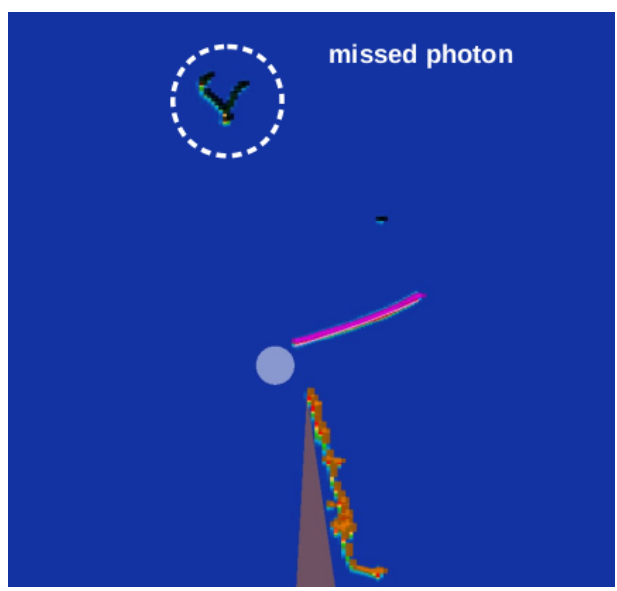

(a) $\pi^{0}$ event with one unreconstructed shower

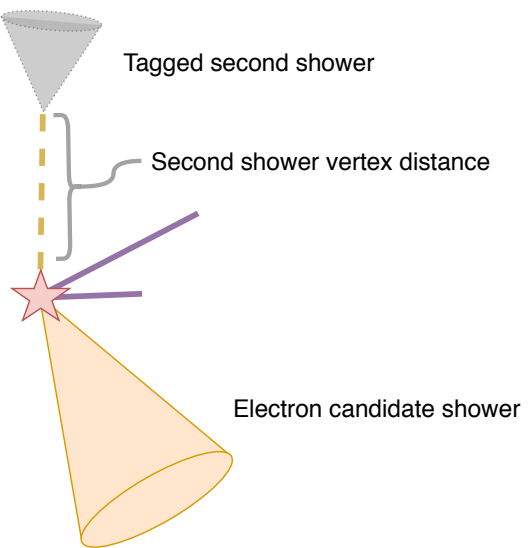

(b) Second shower variables

Figure 8.12: Visual representation of the $\pi^{0}$ rejection variables of the second shower. (Left) Example event where the second shower in a $\pi^{0}$ event is clustered in $2 D$ (black hits) but not fully reconstructed as a shower. (Right) Reconstructed variables associated to the second shower search. The grey cone in the image represents the black cluster on the left image, for which only $2 D$ information is accessible.

of input variables to a binary output, trained using a labelled data-set. In this chapter, the labelled data-set purely consists of simulated Overlay samples, and the label relies on truth information obtained by the backtracking procedure (Section 4.2.4).

We will refer to $y_{i}$ as the binary truth-based label, and $\vec{x}_{i}$ as the collection of input variables, called features, of the model. Here, the subscript $i$ refers to a specific example in the training data-set. The model $\theta$ used to approximate the label $y_{i}$ by the class probability $\hat{y}_{i}$ can be written as:

$$
\hat{y}_{i}=\theta\left(\vec{x}_{i}\right)=\sum_{k=1}^{K} f_{k}\left(\vec{x}_{i}\right), \quad f_{k} \in \mathcal{F}
$$

where $K$ is the number of trees and $f$ is a functional representation of a single tree. $\mathcal{F}$ represents the function space of classification trees up to a certain set of constraints such as the depth and the number of leaves of the tree. 
Table 8.2: Input variables used for electron identification by the BDT. The data/MC distributions corresponding to the variables listed is given in Figure 8.16

\begin{tabular}{|c|c|}
\hline Variable goal & Variable definition \\
\hline \hline$e / \gamma$ separation & $\begin{array}{c}\text { Shower vertex distance } \\
\text { Shower } \mathrm{d} E / \mathrm{d} x \text { at the start of the shower: } \\
- \text { On the collection plane only. } \\
- \text { Weighted mean over the three planes. } \\
- \text { Collection plane, shifted by } 1 \mathrm{~cm} .\end{array}$ \\
\hline$\mu$-rejection & $\begin{array}{r}\text { Shower subclusters } \\
\text { Moliere angle }\end{array}$ \\
\hline$\pi^{0}$ tagging & $\begin{array}{c}\text { Second shower number of hits } \\
\text { Second shower vertex distance }\end{array}$ \\
\hline
\end{tabular}

The objective function used to optimise the model by training consists of two components:

$$
\operatorname{obj}(\theta)=\sum_{i=1}^{N} \mathcal{L}\left(y_{i}, \hat{y}_{i}\right)+\sum_{k=1}^{K} \Omega\left(f_{k}\right)
$$

here, $N$ is the number of elements in the training set.

- $\mathcal{L}\left(y_{i}, \hat{y}_{i}\right)$ is the loss function which measures how predictive our model is with respect to the training data. For the binary classification used in this work, the logistic loss function is used:

$$
\mathcal{L}\left(y_{i}, \hat{y}_{i}\right)=-\left(y_{i} \log \left(\hat{y}_{i}\right)+\left(1-y_{i}\right) \log \left(1-\hat{y}_{i}\right)\right)
$$

The cost function is 0 if the prediction and truth labels are identical, the higher the cost function, the worse the prediction.

$-\Omega\left(f_{k}\right)$ is the regularisation term and controls the complexity of the model. Higher values of $\Omega$ correspond to more degrees of freedom in the model. Including this term in the minimisation of the objective function avoids over-fitting.

The XGBoost training process aggregates an ensemble of weak individual models to obtain a more accurate final model. Gradient boosting involves identifying the shortcomings of weak models and incrementally building a final ensemble model using an objective func- 
tion that is optimised with gradient descent, as illustrated in Figure 8.13.

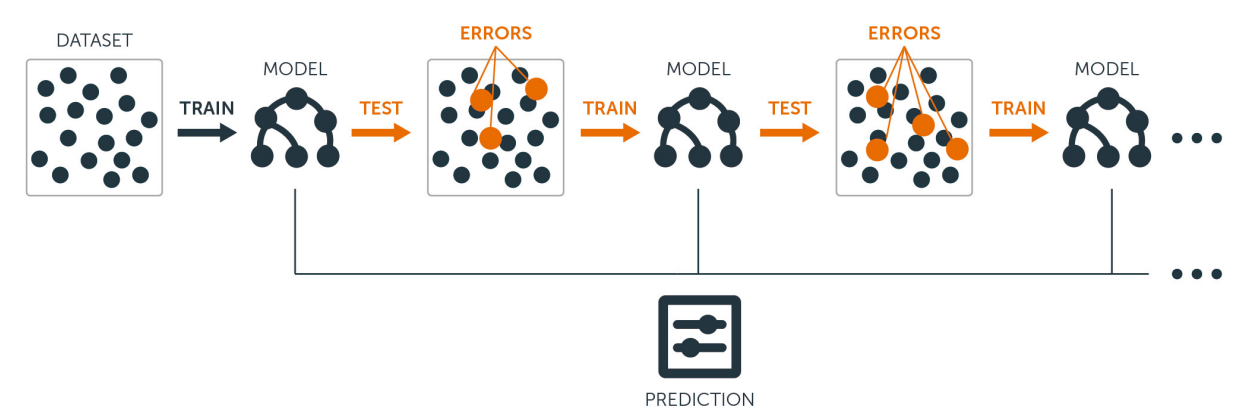

Figure 8.13: Flowchart representing the iterative aspect of the gradient boosting training cycle. Each iteration is called a training epoch. Figure from [102].

\subsubsection{Boosted decision tree performance}

After the preselection, the object that is tagged as the electron candidate is still not a neutrinoinduced electron in most events. The ratio of photons to electrons is approximately 20 to 1 and photon-electron separation is therefore the main objective of electron identification. The identification is done using a boosted decision tree, trained on the variables introduced in Section 8.3.2, summarised in Table 8.2.

The binary classification tree tries to distinguish backtracked electron showers from background showers, dominated by photons. The training and testing is fully performed on a cocktail of simulated Overlay events. This mixture consists mostly of BNB-like neutrino events, enriched with electron neutrino interactions and weighted to compensate the energy dependence of the beam flux.

The outcome of the training process - the BDT response - and validation is given in Figure 8.14. The different panels in the figure from left to right:

- The score of the BDT for the test fraction of the simulated events, divided in the two previously defined categories using truth labelling.

- The Receiver Operating Characteristic (ROC) curve. This metric shows the trade-off between a low false positive rate and a low false negative rate. Each point along the 

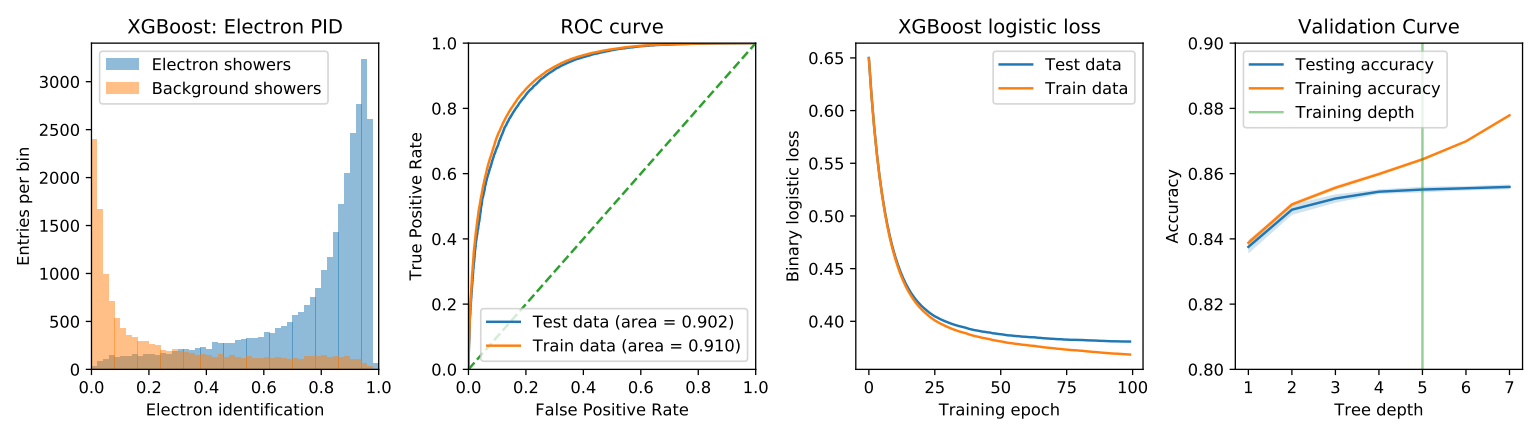

Figure 8.14: Evaluation of training performance for electron shower identification. Different panels explained in the text.

curve corresponds to a certain threshold on the score. The comparison between the test and training set is made, the smaller this difference, the lower the over fitting. The Area Under the ROC Curve (AUC) is a widely accepted metric for the performance of the classifier.

- The binary logistic loss of the classifier on both the training and the test set. The number of epochs is chosen such that the test data has reached an optimal value and stays stable. A large separation between the test and the training loss, or an upwards tendency of the test loss are indications of over-fitting.

- The accuracy as a function of the tree depth. The accuracy is the fraction of correct predictions at a score threshold of 0.5 . The three depth is optimised based on the validation curve to minimise over-fitting while maintaining a high accuracy.

The data/MC comparison of the BDT is shown in the right panel of Figure 8.16c. Although the $p$-value obtained from the KS-test is reasonable, it should be noted that in the signal region, the MC over predicts the data. This is related to the data/MC discrepancies which are observed in the $\mathrm{d} E / \mathrm{d} x$ variables, shown in Figure 8.16a. Currently, a campaign within the MicroBooNE collaboration to re-calibrate the calorimetric properties of reconstructed objects is ongoing. As previously discussed, the $\mathrm{d} E / \mathrm{d} x$ variables are crucial to separate electron and photons and therefore directly impact the main physics goal of MicroBooNE; addressing the origin of the LEE excess as observed by MiniBooNE. Therefore, it was decided to include the variables in this work. Furthermore, the errors shown in the figures in 
Chapters 6 to 8 are statistical only and any discrepancies could be explainable by systematic uncertainties which will be discussed in Chapter 9 .

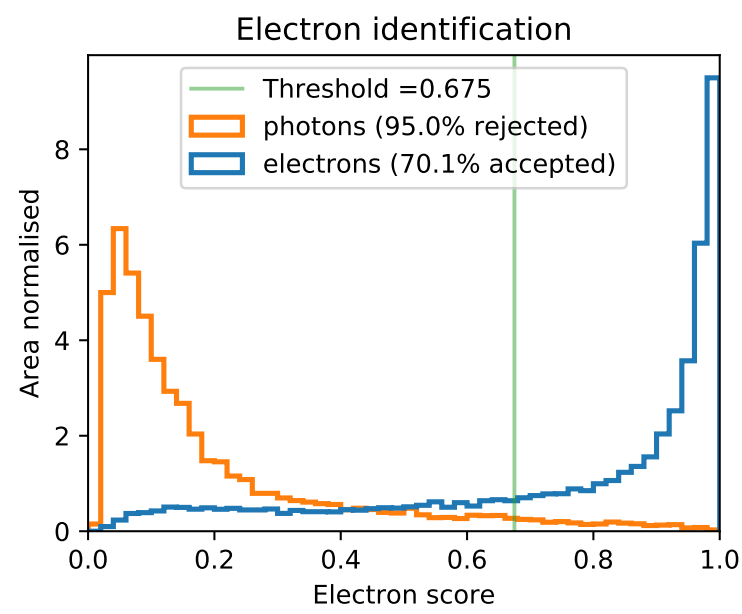

Figure 8.15: $e-\gamma$ separation performance obtained by the electron classifier on simulation. The BDT electron score is shown for true electrons (blue histogram) and for background photons (orange histogram). The green lines indicated the score at which $95 \%$ of the background photons are rejected.

Figure 8.15 addresses the question of how well MicroBooNE can perform $e-\gamma$ separation by using the BDT score. Reco-truth matching is used to subdivide electron candidate showers into showers originating by a $v_{e} \mathrm{CC}$ produced electron and photon showers originating from $\pi^{0}$ decay. It is found that one can reject $95 \%$ of photon showers, while keeping $70 \%$ of electron showers, corresponding to a $15: 1$ increase of signal-to-background ratio.

To conclude this section, in Figure 8.17, the correlation between the variables used to obtain the electron identification score, the score itself, the reconstructed lepton kinematics and the truth-based lepton kinematics are shown for $v_{e} \mathrm{CC}$ electrons, passing the preselection. As expected, the three different $\mathrm{d} E / \mathrm{d} x$ variables are highly correlated. Furthermore, the score strongly prefers showers with a smaller vertex distance, smaller Moliere angle, and larger number of sub-clusters. The correlation with the second shower tagging variables demonstrate that the score prefers events in which no nearby second shower with a substantial amount of hits can be found. The lack of correlation between the electron score and the $\mathrm{d} E / \mathrm{d} x$ variables is expected from the definition of the correlation - Equation (7.8) - since 
electrons have a specific $\mathrm{d} E / \mathrm{d} x$ expectation of $\approx 2.1 \mathrm{MeV} \mathrm{cm}^{-1}$, instead of low / high values.

Finally, it is encouraging to see that the correlation between the electron score and the truthbased kinematics is low. This serves as a further demonstration that the electron identification is kinematically unbiased, covering an as large as possible portion of the lepton kinematics phase space. The lepton kinematics, their resolution and systematic uncertainties will further be discussed in Chapter 9. 

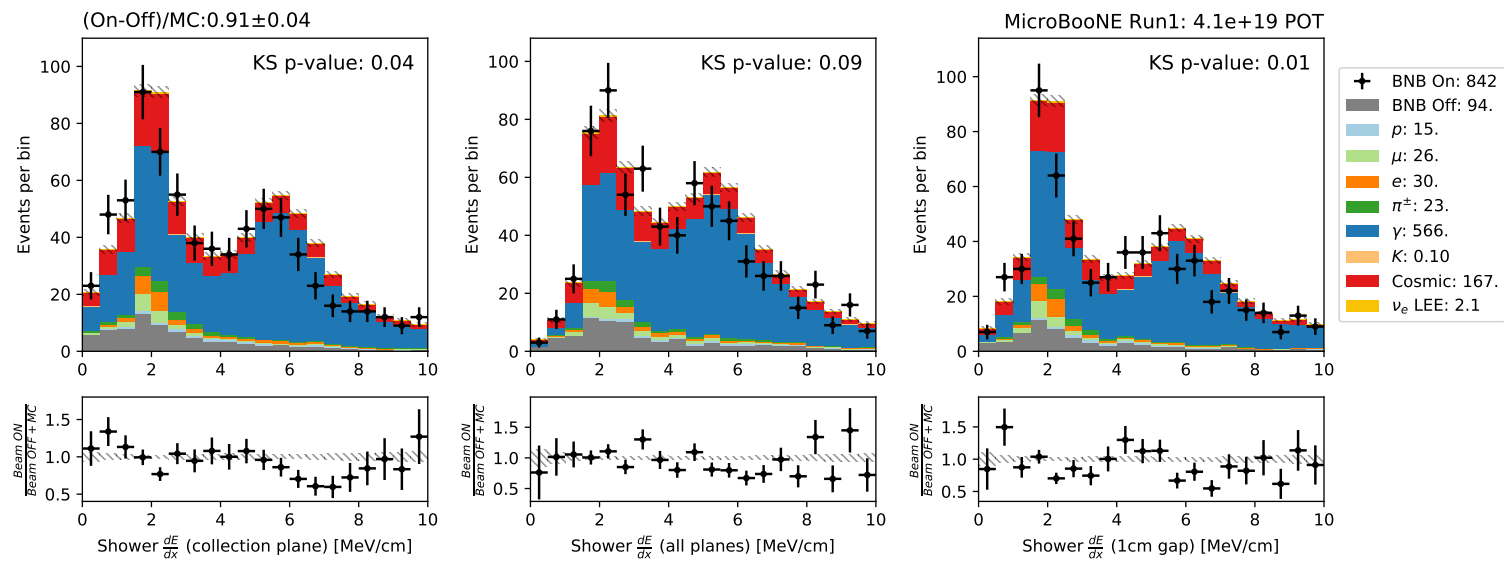

(a) The three variables used to characterise the $\mathrm{d} E / \mathrm{d} x$ at the start of the electron candidate shower.
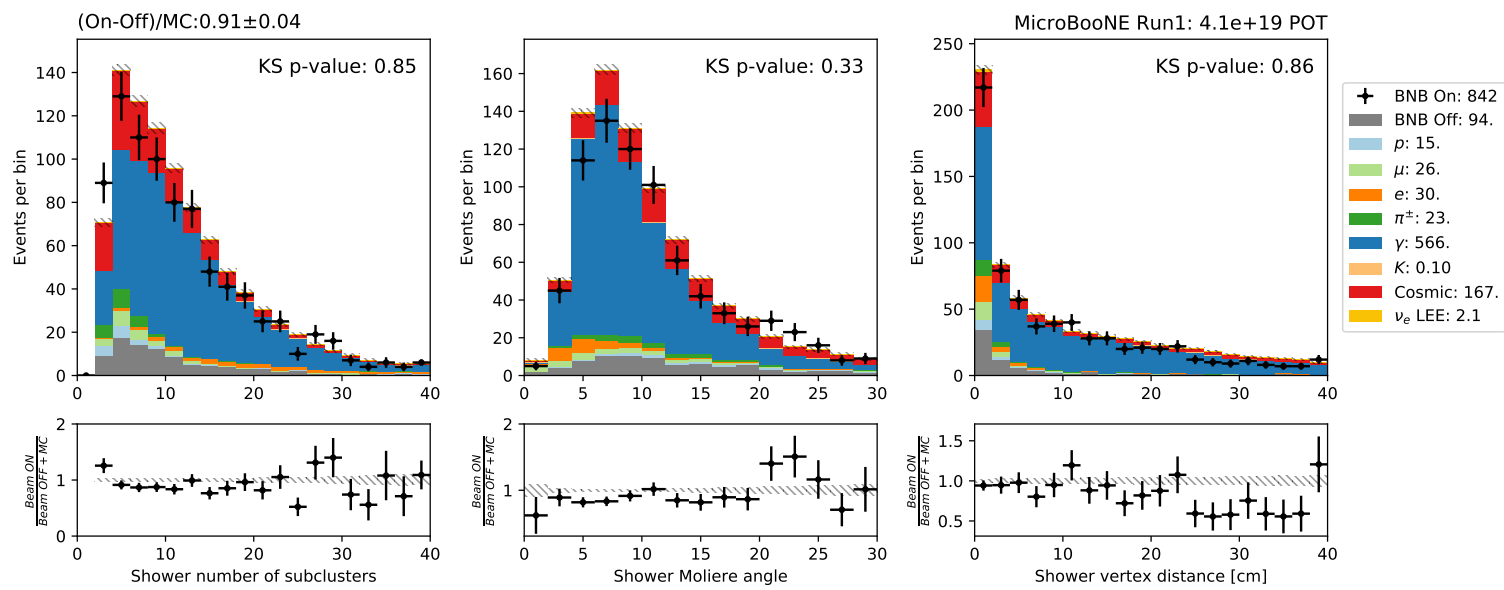

(b) Geometrical properties and the shower vertex distance of the electron candidate shower.
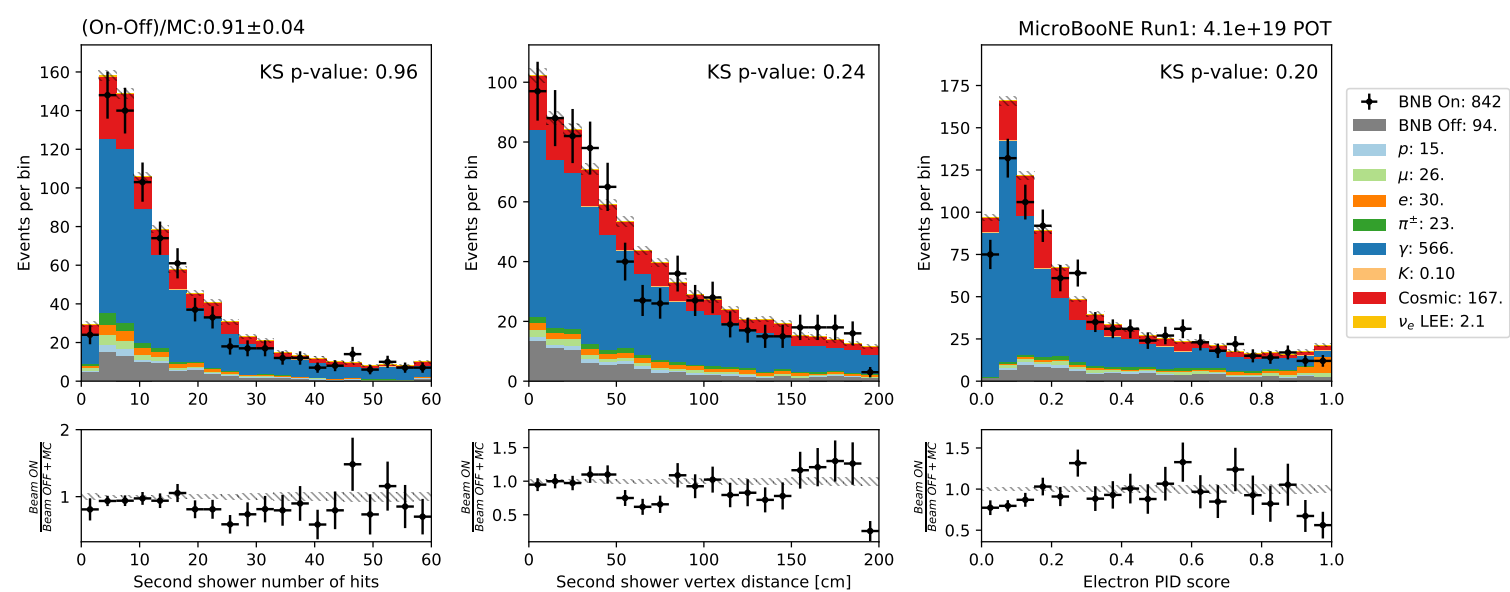

(c) Second shower tagging variables and the electron PID score obtained from the BDT.

Figure 8.16: Data/MC comparisons for the input variables of the electron shower classifier, as listed in Table 8.2 and its response (bottom right panel). 


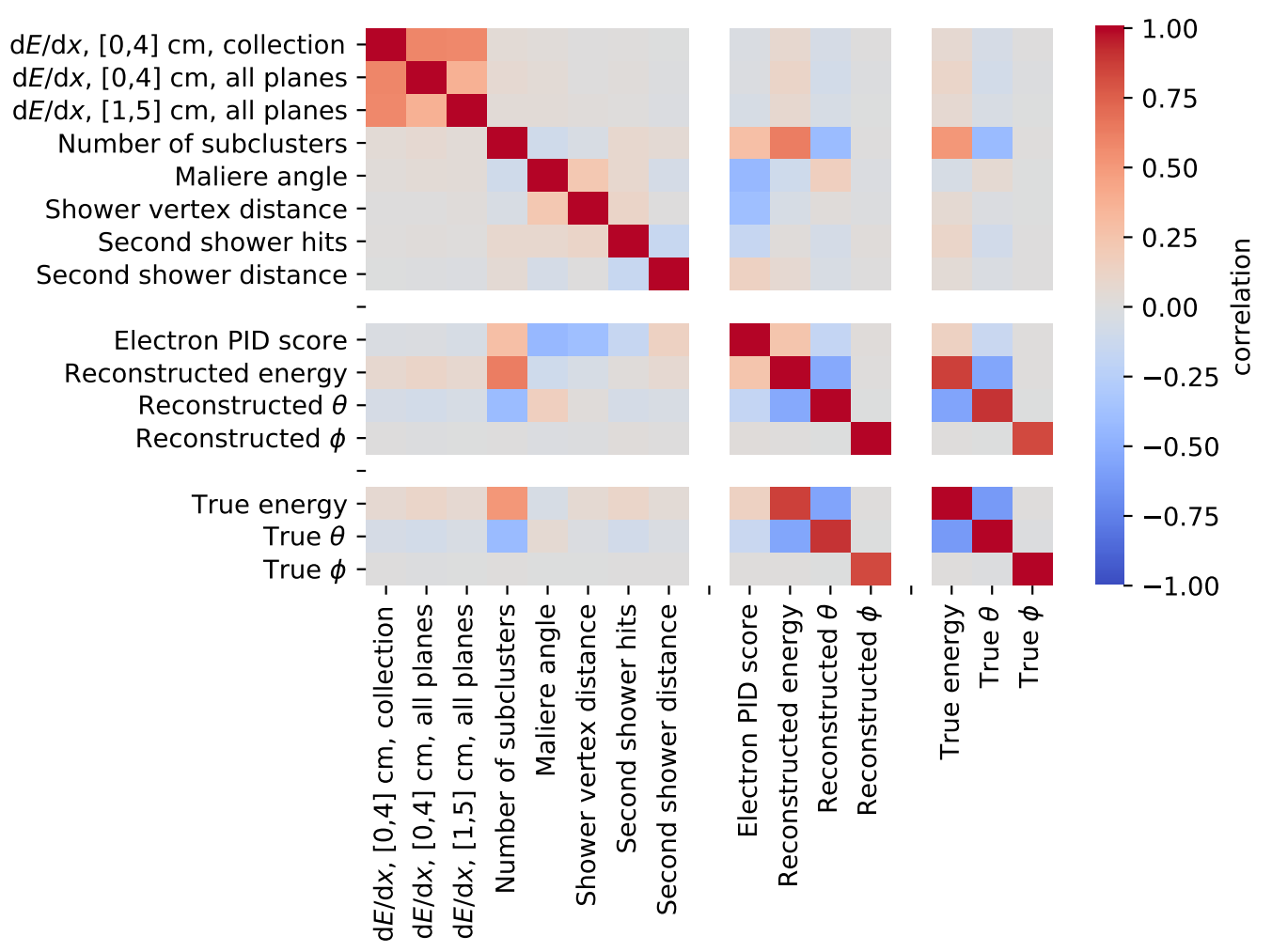

Figure 8.17: Correlation matrix of the variables of interest in the electron identification step. The first group (top-left) contains the variables used in the electron PID. The second group (middle) are the BDT score and the reconstructed lepton kinematics that can be compared with the backtracked counterparts in the third group (bottom-left). 


\subsection{Muon neutrino induced backgrounds}

Besides the electron candidate, the neutrino slice includes a number of particles which can be leveraged to improve background rejection (see Figure 8.2). These particles are referred to as other daughters, stressing they are part of the reconstructed neutrino particle hierarchy and exclude the electron candidate particle, which was discussed in the previous section.

We classify each other daughter using the a separate BDT. This BDT is trained on three labelled categories: signal-like, neutral and background-like.

- The signal-like particles are those neutrino daughters that are backtracked to a proton or a split-off part of the electron. It is clear that these particles might be part of a $v_{e} \mathrm{CC}$ interaction and should not be used to reject events.

- The background-like particles are those neutrino daughters that are backtracked to a simulated muon or cosmic activity. Events that contain any of these should be rejected.

- The "neutral" label accounts for ambiguous cases: this label is given to reconstructed particles backtracked to charged pions or photons. Charged pions should be allowed in a $v_{e}$ CC search, but the mis-identification rate between charged pions and muons is large in LArTPCs due to their similar ionisation profile. Similarly, photons arise from neutral pions can be part of a $v_{e}$ CC interaction, but can help eliminating NC $\pi^{0}$ backgrounds too.

The variables used to train the classifier are listed in Table 8.3 an their data/MC distributions are given in Figures 8.19a and 8.19b. The first variable is the track log likelihood PID which was introduced in Section 7.3.1. In principle, if there is a final state electron, there can be no final state muon in the interaction. ${ }^{1}$ Therefore, one could argue that electron identification would render muon rejection redundant. On the contrary, due to the photon background which dominates the electron contribution by over an order of magnitude,

\footnotetext{
${ }^{1}$ More esoteric processes could produce an electron and a muon in $v_{\mu} \mathrm{CC}$ interactions. However, the rates at which this occurs is negligible in MicroBooNE.
} 
additional muon-rejection is essential to reduce the $v_{\mu} \mathrm{CC} \pi^{0}$ backgrounds. As was demonstrated in Section 7.3, the track log likelihood PID has a very good muon-proton separation.

The next two variables are used to perform additional $\gamma$-tagging. Additional showers apart form the electron candidate shower - can either be parts of the electron shower, or arise from neutral pion decay. These objects are identified using the track score and the distance between the reconstructed neutrino interaction vertex and the start point of the daughter.

The last three variables are related to the Pandora neutrino hierarchy, which was introduced in Section 4.2.3. Reconstructed particles can be direct daughters of the neutrino (second generation) but they can also be daughters of those daughters (third generation). Three boolean variables tailored to specific physics cases are introduced:

- Has shower-like daughter: In combination with a high track score and high track log likelihood PID, this indicates the presence of a Michel electron, reconstructed as a showerlike daughter. These events can be rejected.

- Has track-like daughter: The presence of a track-like particle which has an additional track-like daughter is a signature of a charged pion decaying into a $\mu^{ \pm}$. Charged pions can be part of $v_{e}$ CC events and should therefore not be rejected.

- Daughter generation: There are several plausible explanations for particles in the third generation. Two were mentioned above: Michel electrons and $\mu^{ \pm \prime}$ s from $\pi^{ \pm}$-decay. A third possibility is that the electron shower was in fact split into multiple objects. The BDT aims to attribute a high score to these objects.

In practice, as can be seen from the left panel in Figure 8.16b, third generation particles are quite rare, and therefore of limited importance for the selection. Nevertheless, LArTPC reconstruction is still evolving and these hierarchical structures will become more important for particle identification as reconstruction performance further improves.

Figure 8.18 illustrates the performance and validation of the other daughter classification. Due to the large training sample and the small amount of variables included, the over- 
Table 8.3: Input variables used for other identification by the BDT. The data/MC distributions corresponding to the variables listed is given in Figures 8.19a and 8.19b.

\begin{tabular}{|c|c|}
\hline Variable goal & Variable definition \\
\hline \hline$\mu$-rejection & Track log likelihood PID \\
\hline$\gamma$-identification & $\begin{array}{c}\text { Vertex distance } \\
\text { Track score }\end{array}$ \\
\hline \multirow{2}{*}{ Particle hierarchy } & $\begin{array}{c}\text { Daughter generation } \\
\text { Has a shower-like daughter } \\
\text { Has a track-like daughter }\end{array}$ \\
\hline
\end{tabular}
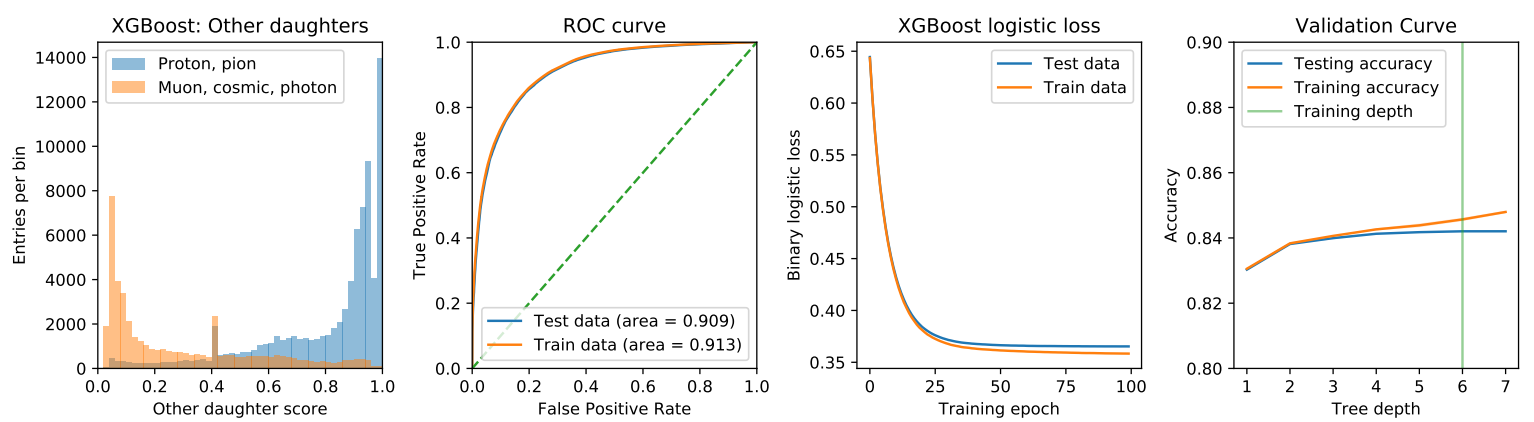

Figure 8.18: Evaluation of training performance for the other daughters in the event. The description of the different panels is given in Section 8.3.4. The spike in the BDT response in the left panel is related to the boolean input variables.

fitting is minimal. The data/MC comparison is given in Figure 8.19c. The left panel shows the score for all daughters. Low scores are given to muons, cosmic-induced particles and photons, higher scores are obtained for protons and electron-shower fragments. In the right panel, the score distribution for the lowest scoring other daughter is given. It can be seen that $v_{e} \mathrm{CC}$ events are located towards the higher end of the distribution, demonstrating the background rejection power of these variables. 

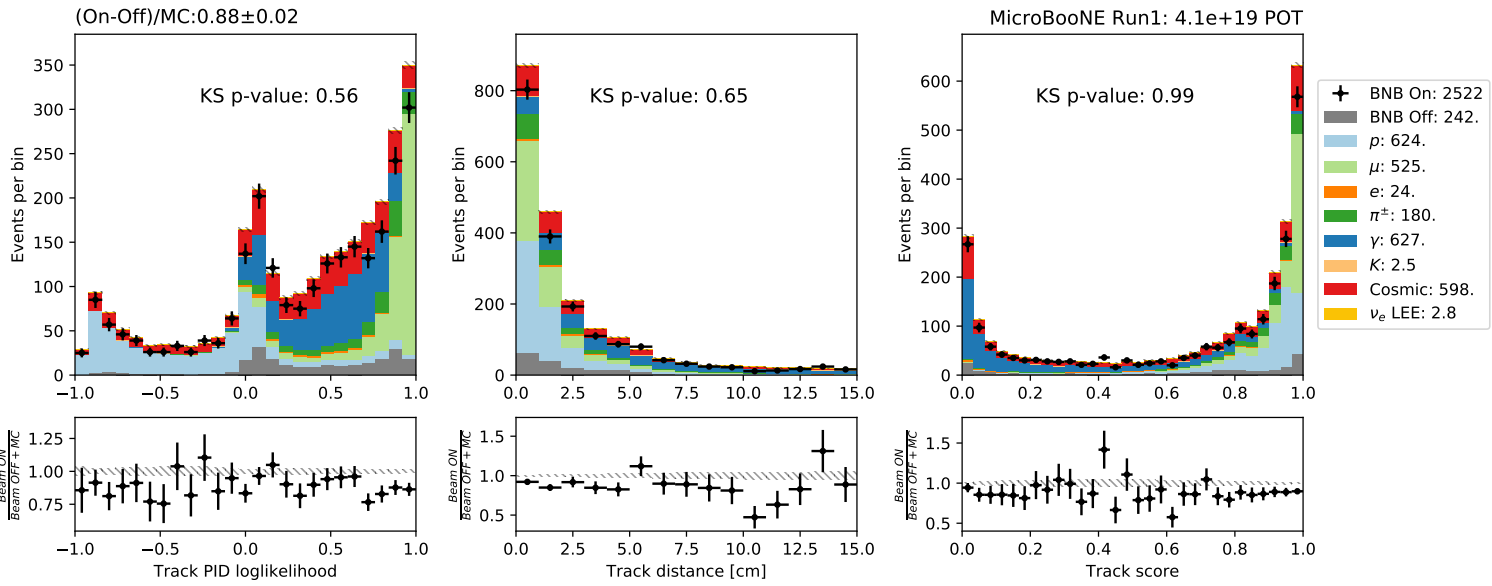

(a) Track related variables: PID log likelihood, vertex distance and track score.
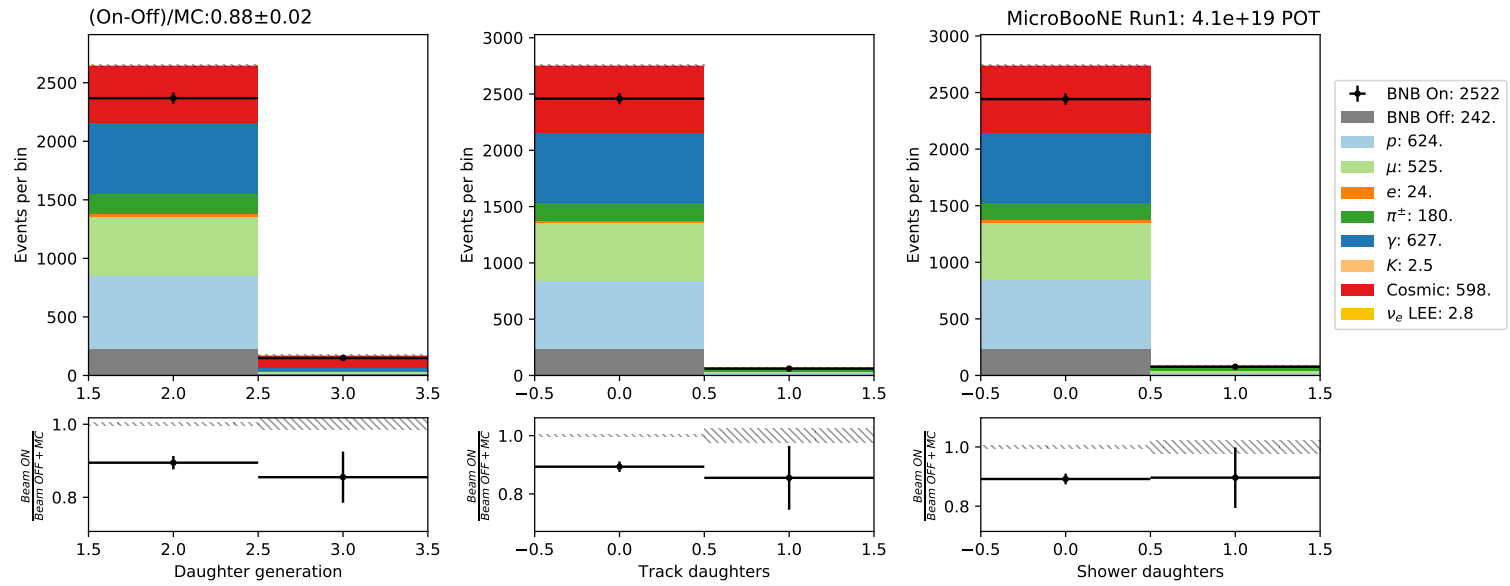

(b) Three boolean variables characterising the hierarchy of the reconstructed neutrino daughters.
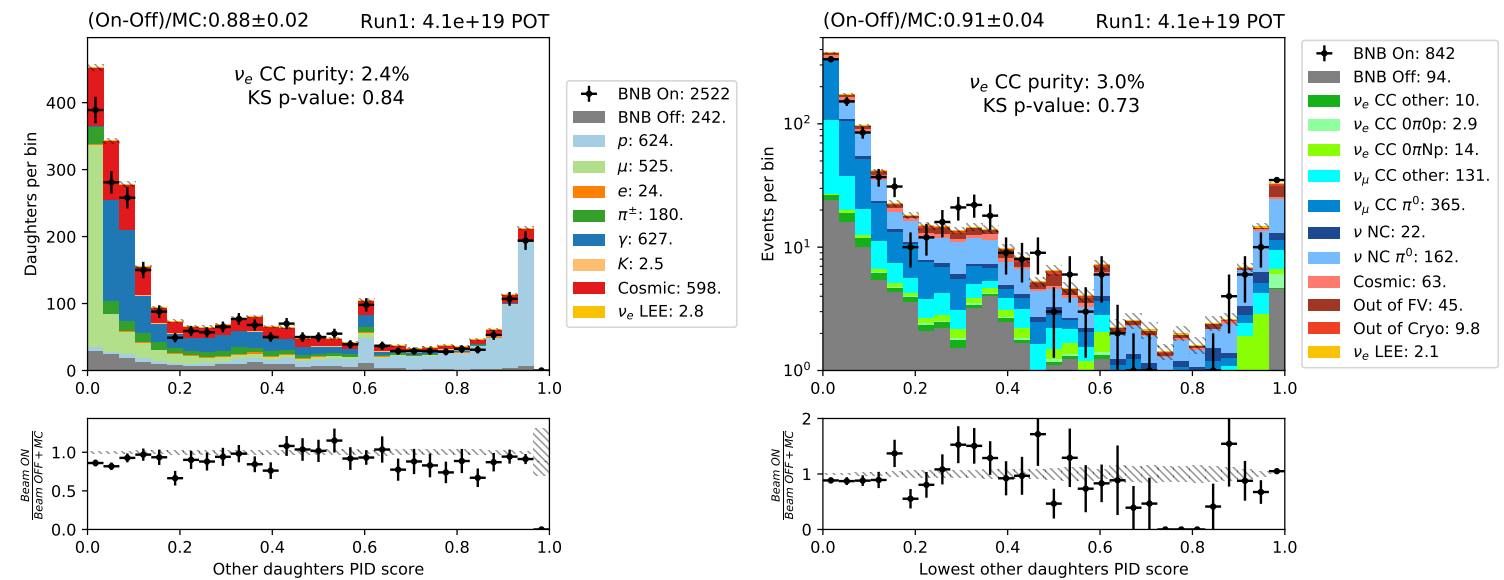

(c) (Left) Response of the classifier for all other daughters in the preselected events. (Right) Lowest score of the other daughters, filled once per event. Note the log scale on the vertical axis.

Figure 8.19: Data/MC comparisons for the input variables of the other daughters classifier. The bottom row shows the response of the BDT 


\subsection{Inclusive $v_{e}$ CC selection}

The final event classification is performed using a BDT building on the particle identification performed in the two previous sections. For this final BDT, the dominant variable is the electron identification response but the full list of variables is, in sequence of importance:

1. Electron shower candidate BDT response.

2. Lowest scoring other daughter score.

3. Number of reconstructed shower-like particles in the event.

4. Ratio of shower-like hits to total hits in the slice. Shower-like hits are defined as the constituent hits of shower-like reconstructed particles (see Section 4.2.3.

5. The mean score of the other daughters classification.

6. Number of reconstructed particles with a vertex distance of $>3 \mathrm{~cm}$.

The distributions of the particle identification related BDT variables were discussed in Sections 8.3.4 and 8.4. The other three distributions are given in Figure 8.21. Completely analogous as to the particle identification BDT, Figure 8.21 illustrates the performance and validation of the event classification.
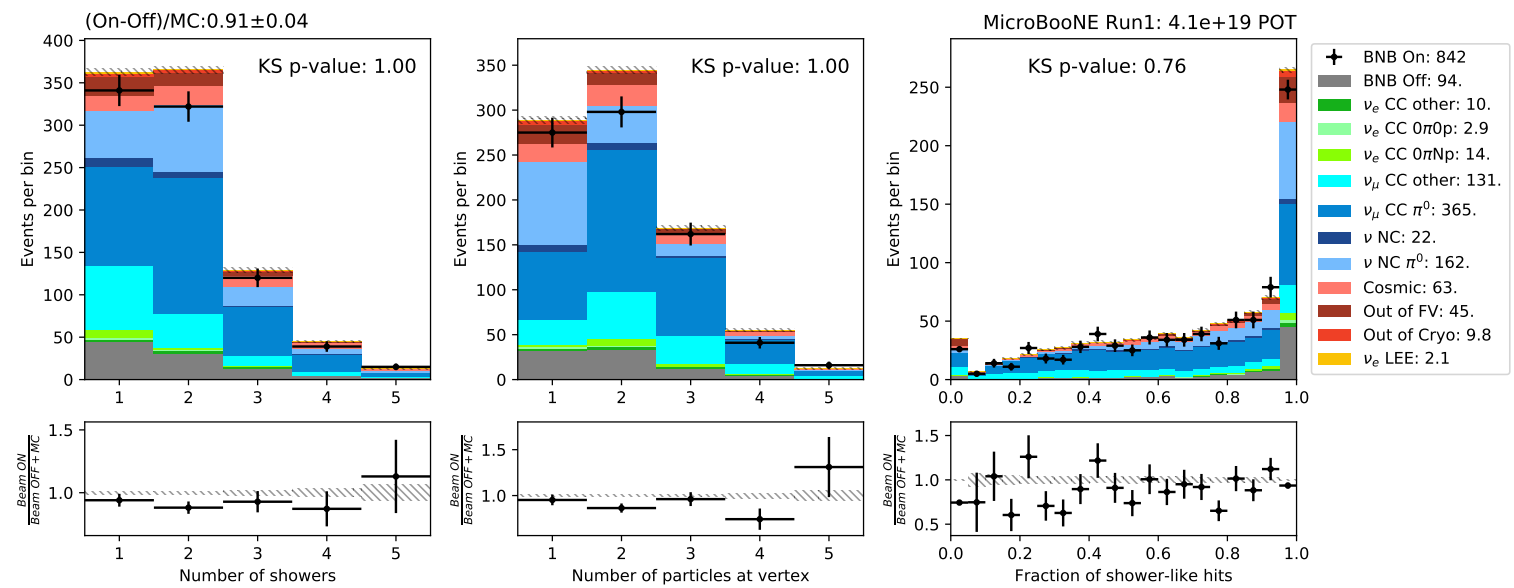

Figure 8.20: Three of the variables used for the final $v_{e} \mathrm{CC}$ event selection classifier. 

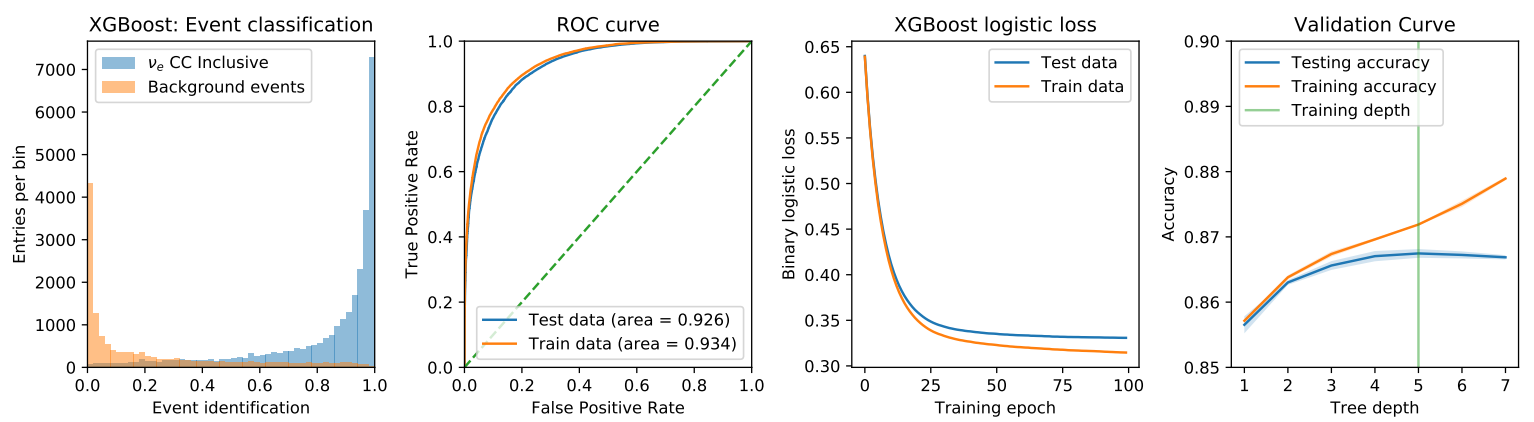

Figure 8.21: Evaluation of training performance for the $v_{e} \mathrm{CC}$ event classification. The description of the different panels is given in Section 8.3.4.

\subsubsection{Purity, efficiency \& remaining backgrounds}

The response of the event classification is given in the left panels of Figure 8.22 for the unblinded data of both data-taking period Run1 (top) and Run3 (bottom). The final $v_{e}$ CC selection includes all events with a score above 0.96 . The $v_{e} \mathrm{CC}$ selection efficiency is $21 \%$, corresponding to approximately 300 electron neutrino's in the full Run 1-4 data-set $\left(1.01 \times 10^{21} \mathrm{POT}\right)$. The distribution of the reconstructed electron shower energy is given in the right panels of Figure 8.22. The relative fraction of Beam Off background is reduced by more than a factor two in Run3 due to the incorporation of CRT tools in the preselection phase (see Sections 6.5 and 8.2). The purity of the selection is $37.6 \%$ without the CRT and goes up to $45.9 \%$ in Run3 when the CRT is included. Furthermore, the dominant background categories after selection are $v_{\mu}$-induced backgrounds, especially those with a $\pi^{0}$ in the final state.

Figure 8.23 documents the efficiency of the NeutrinoID, preselection and final inclusive selection for $v_{e}$ CC interactions. At the end of this chapter, a set of MicroBooNE Beam On events displays is included, Figure 8.25. These events are a subset of the events populating the right panels of Figure 8.22 and aim to demonstrate the variety of final states and extent of the energy range that is covered by the selection. 

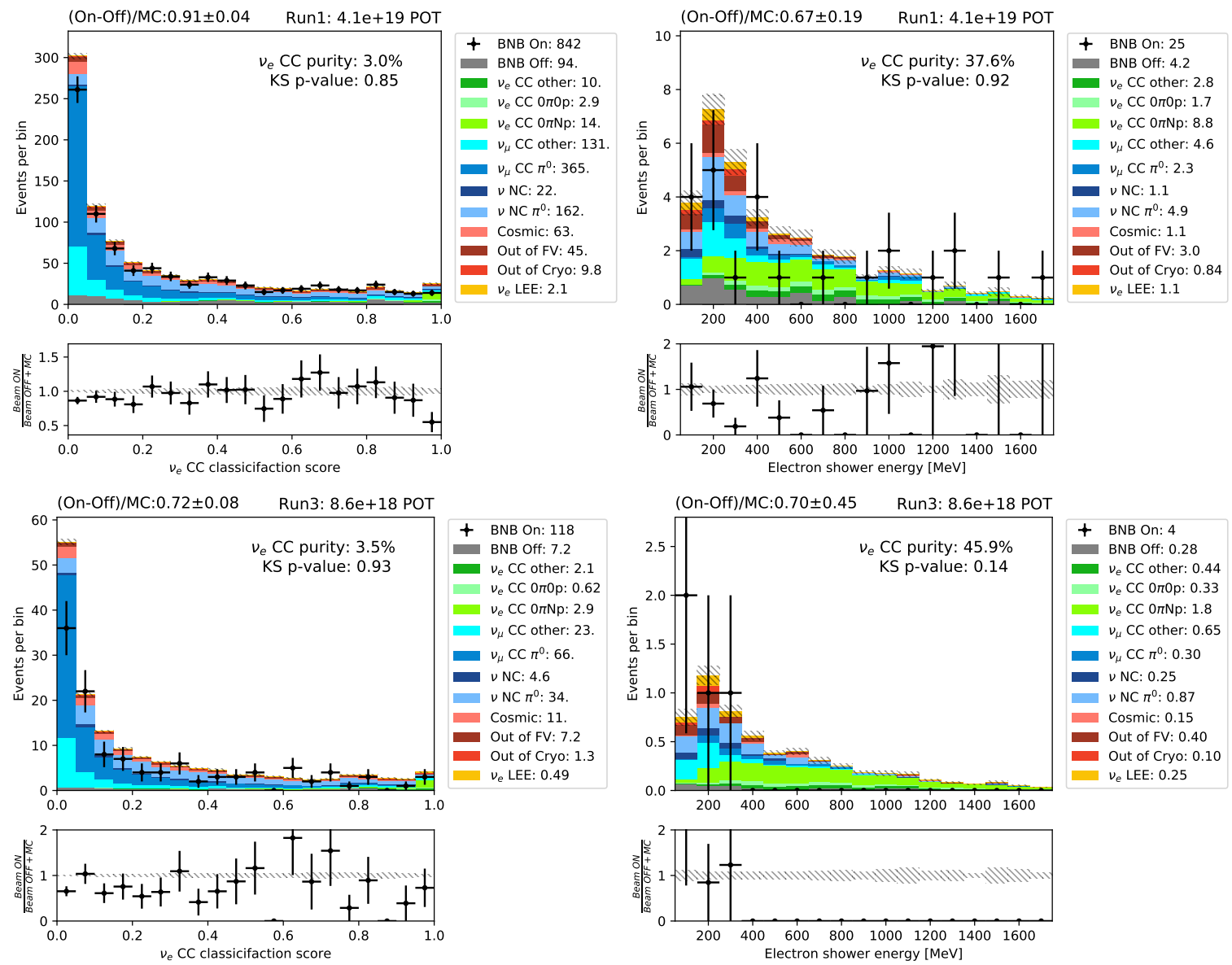

Figure 8.22: (left) BDT response of the $v_{e}$ CC inclusive event classifier. (right) Reconstructed electron shower energy distribution after the selection. The top panels correspond to the unblinded portion of data from Run1, the bottom panel used data collected during the third run period. The relative contribution from cosmic-related and out-of-fiducial volume backgrounds is reduced thanks to the incorporation of the CRT tools.

\subsubsection{Limitations of the reconstruction framework}

The final efficiency - integrated over the lepton kinematics - of the $v_{e}$ CC selection is $21 \%$. In some important corners of the phase space, such as low-energy events and backward going electrons, the efficiency is lower due to reconstruction effects [73]. Nevertheless, the $v_{e}$ CC selection outlined in this thesis has the highest efficiency $\times$ purity in any electron neutrino selection performed in a LArTPC to date. In both Chapter 6 and in this chapter, the inefficiencies in different steps were mentioned. In Figure 8.23 the efficiency in the three stages of the selection - the NeutrinoID, the preselection and the final selection -is 

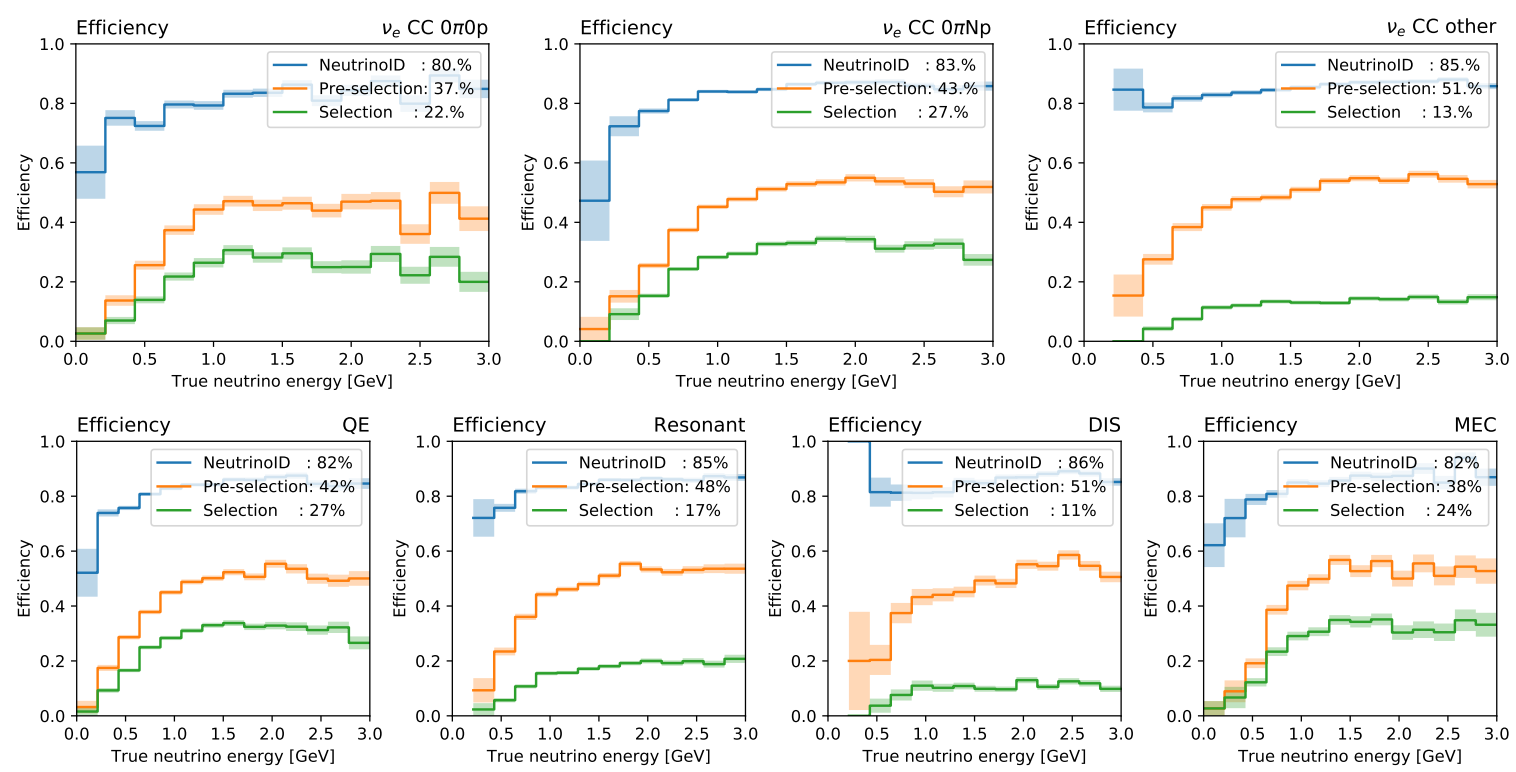

Figure 8.23: Efficiency of the different stages in the $v_{e} \mathrm{CC}$ selection as a function of the true neutrino energy. The three panels in the top row correspond to different final state topologies, the panels in the bottom row show the efficiency for different interaction modes that were introduced in Section 2.5. The uncertainties on the integrated efficiency in the legend is below $1 \%$ for all cases. The NeutrinoID (blue) is flat at neutrino energies above $\mathcal{O}(1 \mathrm{GeV})$ as was shown more clearly in Figure 6.20.

shown. However, by only looking at the loss in efficiency step by step, the overview might be lost. Therefore, in this paragraph, an approximate upper limit of the efficiency that can be archived with the current tool-set is estimated using truth information.

Figure 8.24 introduces a set of reconstruction performance metrics. The events used to evaluate the reconstruction performance are simulated $v_{e}$ CC Overlay events that passed the NeutrinoID. Therefore, the blue curve in Figure 8.23 is taken as the denominator.

Electron shower reconstruction To select $v_{e}$ CC events, a perfect electron tagging algorithm in combination with a Michel electron energy threshold cut would effectively lead to zero backgrounds. Therefore, electron reconstruction is key. The top-left panel in Figure 8.24 shows the electron reconstruction efficiency as a function of energy. An electron is considered reconstructed if it has a purity and completeness of at least $50 \%$ and is reconstructed as a shower-like object. Note that $50 \%$ is a very loose request and that tricks as re-clustering 
and shower merging might be needed to improve the energy resolution. With this reconstruction definition, $\approx 75 \%$ of electrons are reconstructed, dropping to $\approx 50 \%$ at low energy.

The same panel also shows the proton reconstruction efficiency for protons with a kinetic energy above $40 \mathrm{MeV}$ that are part of $v_{e} \mathrm{CC}$ interactions. For the right panel of Figure 8.1, one can see that only for neutrino energies $\lesssim 500 \mathrm{MeV}$ the $v_{e} \mathrm{CC} 0 \pi 0 \mathrm{p}$ becomes important. However, the reconstruction efficiency of protons needs to be taken into account. The observed proton reconstruction efficiency of approximately $60 \%$ moves a significant fraction of $v_{e} \mathrm{CC} 0 \pi 1 \mathrm{p}$ events into the single electron shower category. The lack of reconstructed vertex activity often deteriorates the vertex reconstruction performance and makes the $e-\gamma$ separation more challenging.

Vertex reconstruction The importance of the vertex reconstruction is strongly related to the $e-\gamma$ separation. First, the start of the shower needs to be well-reconstructed to differentiate the $\mathrm{d} E / \mathrm{d} x$ at the start. The double ionising part of the photon shower in the case of pair production only extends for $\approx 4 \mathrm{~cm}$. Second, reconstructing the neutrino vertex and/or the electron shower start point at the wrong position will lead to an effective vertex displacement. This vertex displacement is a characteristic of photons and might therefore lead to a higher rate of mis-identification. The resolution of the reconstructed neutrino vertex and electron start point are given in the top-right panel of Figure 8.24. In approximately $50 \%$ of the electron showers, the displacement is above $3 \mathrm{~cm}$. Note that for simulated $v_{e} \mathrm{CC}$ events, the true shower start point coincides with the neutrino interaction vertex. On the contrary, the reconstructed neutrino vertex and shower vertex do not have to be in the same location.

Slice reconstruction To properly reconstruct the final state particles and estimate the energy, the slice should have a high purity and completeness. This means that most neutrino induced hits are clustered inside the slice and that the amount of hits inside the slice due to unrelated cosmic activity is as low as possible. As is shown in the bottom-left panel of Figure 8.24 , the fraction of events with high hit-purity is reasonable, but only $60 \%$ of the events have a slice with a high completeness. This means that in practice, a lot of the deposited 
energy is not available for downstream analysis. The bottom-right panel shows these slice quality metric as a function of the neutrino energy. It can be concluded that less than $50 \%$ of the low-energy $v_{e} \mathrm{CC}$ events are reconstructed with high purity and efficiency. A more in depth discussion of reconstruction limitations can be found in [73].

One can now combine all the previous truth-based requirements. Requiring that the reconstructed $v_{e} \mathrm{CC}$ event has:

- A reconstructed shower backtracked to the electron.

- A neutrino interaction vertex and shower start point reconstructed within $3 \mathrm{~cm}$ from the simulated neutrino interaction point.

- A slice with a purity and completeness of neutrino-induced reconstructed hits of at least $80 \%$.

These well-motivated requirements are only fulfilled in $26 \%$ of the $v_{e}$ CC events. As stated in the beginning of this section, these studies have been performed after the neutrino slice is identified by the NeutrinoID (Chapter 6). Taking into account the NeutrinoID efficiency, we find that $21.5 \%$ of the events are well-reconstructed. ${ }^{2}$ Inside this sub-sample of events, the selection described in this chapter has an efficiency of approximately $60 \%$, three times higher than on the full $v_{e}$ CC signal sample. Therefore, if can be concluded that the main gains in efficiency need to be sought in the early reconstruction stage.

\footnotetext{
${ }^{2}$ This fraction should be taken as an estimate and depends strongly on how well-reconstructed is defined. This definition depends on the physics goals of a specific selection.
} 

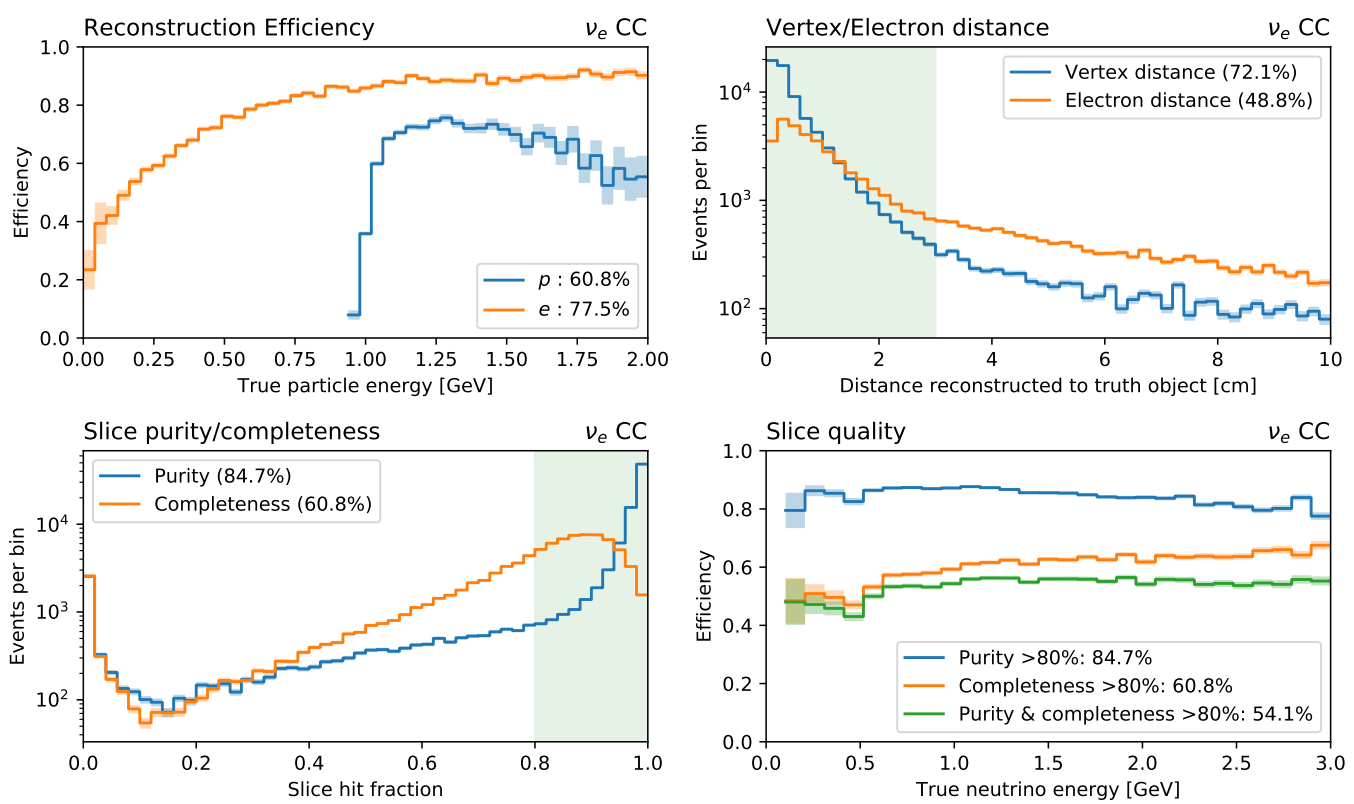

Figure 8.24: Reconstruction quality metrics for $v_{e}$ CC signal events passing the NeutrinoID. The details of the different panels are discussed in the text. 

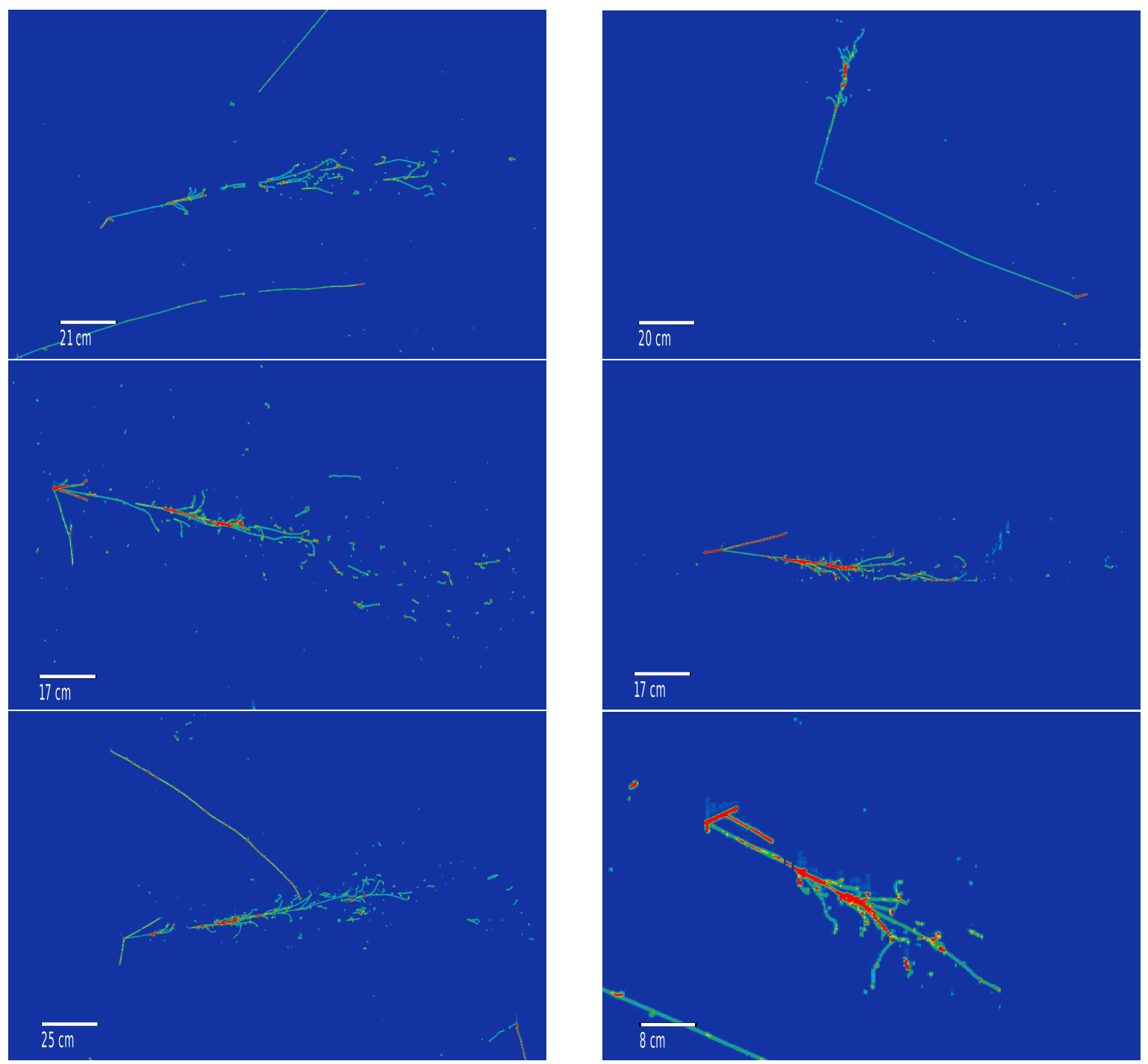

(a) Selected $v_{e}$ CC candidates as seen on the $Y$ plane, Run1 $\left(4 \times 10^{19}\right.$ POT $)$ data-set. Reconstructed shower energies, from left to right, top to bottom: $965 \mathrm{MeV}, 204 \mathrm{MeV}, 1086 \mathrm{MeV}, 989 \mathrm{MeV}, 1444 \mathrm{MeV}$ and $754 \mathrm{MeV}$
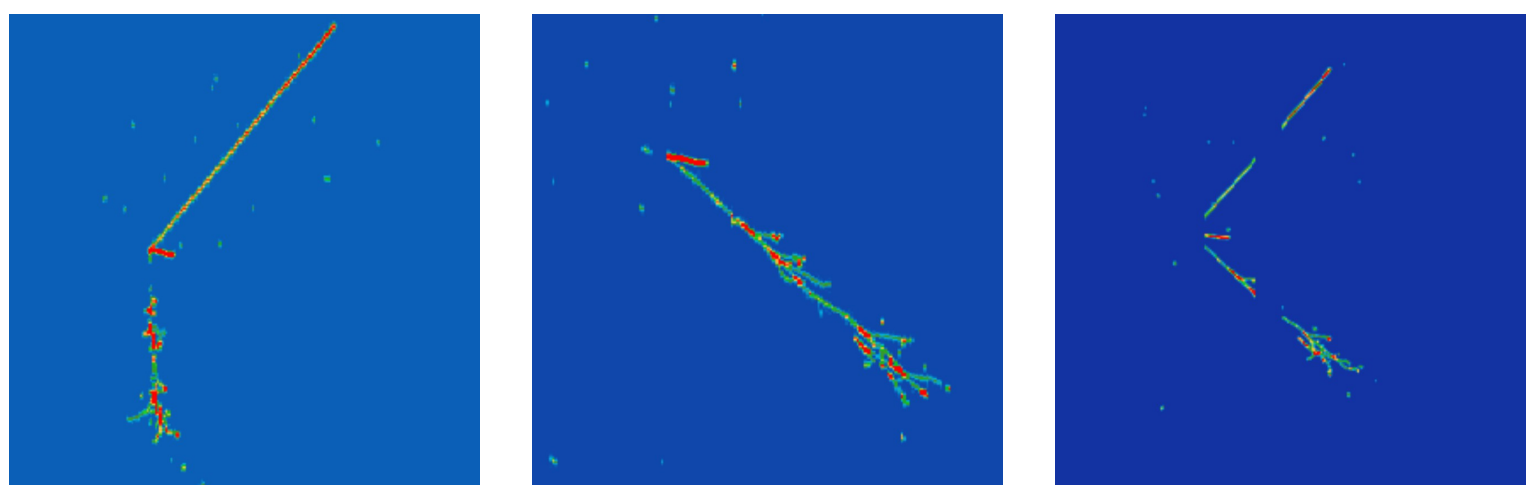

(b) MicroBooNE electron neutrino candidate from the $9 \times 10^{18}$ POT Run3 data-taking period. The reconstructed shower energy is $297 \mathrm{MeV}$. The three planes, $U, V$ and $Y$ are given from left to right.

Figure 8.25: Displays of selected $v_{e} \mathrm{CC}$ candidate events by the selection described in this chapter. 
Chapter 8. Charged-Current Electron Neutrino Selection 


\section{9 \\ Data-Driven $v_{\mu}$ Constraint \& Charged-Current $v_{e}$ Measurement with the BNB}

In order to make statements on whether the observed $v_{e} \mathrm{CC}$ rate and corresponding distributions of the lepton kinematics indicate the presence of potential new physics, a well understood prediction of the BNB intrinsic $v_{e}$ interaction rate in the MicroBooNE TPC is needed.

In this chapter, we describe the main sources of systematic uncertainties which affect the $v_{\mu} \mathrm{CC}$ and $v_{e} \mathrm{CC}$ selections developed in Chapters 7 and 8. Uncertainties in the expected neutrino interaction rates are associated with modelling uncertainties, as well as reconstruction efficiency variations caused by detector effects. The systematic uncertainties related to the modelling are separated into $v_{e}$ flux and neutrino-argon cross-section predictions, 
treated in Sections 9.1.1 and 9.1.2 respectively.

Afterwards, in Section 9.2, it is demonstrated how the $v_{\mu}$ CC measurement can be used as a data-driven constraint to reduce the systematic uncertainties in the $v_{e} \mathrm{CC}$ measurement. Section 9.3.2 introduces statistical techniques to characterise the intrinsic electron neutrino content in the Booster Neutrino Beam. Finally, Section 9.3.3 investigates the statistics-only sensitivity of the selection to possible new physics in a $3+1$ sterile neutrino scenario.

\subsection{Sources \& treatment of systematic uncertainties}

The $v_{\mu} \mathrm{CC}$ and $v_{e} \mathrm{CC}$ selections are subject to some level of uncertainty, both statistical and systematic. The systematic uncertainties are associated to the knowledge of the neutrino beam (flux), the level of precision of the model used in the simulation of neutrino interactions (GENIE) and modelling of the detector response (detector systematics). These systematic uncertainties can be evaluated for many parameters of the models and measured variables that are used in the event reconstruction and selection.

When treating systematic uncertainties, one wishes to take into account correlations between different bins of the distribution of a given reconstructed variable. For each uncertainty, a covariance matrix is constructed, correlating the variation in the measured number of events between two bins. Two approaches are followed to obtain the covariance matrix, depending on the type of uncertainty: unisim or multisim.

Unisim means that a single variation of a given analysis input parameter is performed according to its uncertainty. The difference in the number of selected events between this variation and the central value is taken as the uncertainty on that number of events. The unisim covariance matrix is defined as:

$$
E_{i j}^{u n i s i m}=\sum_{k}^{V}\left(N_{i}^{C V}-N_{i}^{k}\right)\left(N_{j}^{C V}-N_{j}^{k}\right)
$$

where $V$ is the total number of detector variation samples, $N_{i}^{k}$ is the value in the $i$-th bin of 
the $k$-th detector variation and $N_{i}^{C V}$ is that of the central value. The square root of the diagonal elements in the covariance matrix are the standard deviations for the individual bins, i.e. $\sqrt{E_{i i}}=\sigma_{i}$. Note that in practise the variations are realised by re-weighting, modifying Equation (9.1) by replacing $N_{i}$ with $\sum_{l} w_{l i}$. Here, the summation is over the $l$ events in bin $i$ with weights $w_{l i}$.

In the multisim procedure, several variations of a given analysis input parameter are performed simultaneously. These are called universes. These different variations are obtained from a different sampling of the input parameter, which is varied within its uncertainty. This approach allows to preserve correlations in the various bins of the distributions of selected events. The covariance matrix can then be obtained as

$$
E_{i j}^{\text {mulitsim }}=\frac{1}{U} \sum_{k}^{U}\left(N_{i}^{C V}-N_{i}^{k}\right)\left(N_{j}^{C V}-N_{j}^{k}\right),
$$

where $U$ is the total number of universes and $N_{i}$ the weighted number of events in bin $i$. The total covariance matrix $E$ is defined as the sum of the covariance matrices related to different sources of uncertainties:

- Simulation of the BNB neutrino flux.

- Modelling of the neutrino interactions in liquid argon.

- Detector variations.

- Limited statistics in the simulated Overlay samples and the Beam Off sample. This statistical uncertainty is included in the figures in Chapters 6 to 8.

- Hadronic re-interaction Uncertainties.

The first three will be discussed in the next three subsections. The last one is related to hadrons interacting strongly with argon nuclei and can be interpreted as GEANT uncertainties in the cross section of a hadron's interaction with argon nuclei. These hadronic re-scatterings can induce a large momentum transfer in the hadron and thus have an impact in the reconstruction and the selection of events. They can lead to a re-classification 
of the event based on the final-state particles produced in the event. The inclusive nature of the selections presented in this work are not dependent on specific final state topologies and therefore minimally impacted by these variations.

The correlation matrix, using the Pearson linear correlation is defined as:

$$
\rho_{i j}=\frac{E_{i j}}{\sigma_{i} \sigma_{j}}
$$

This matrix provides a measure of the correlation of a variation affecting bin $i$ and bin $j$. The value of $\rho_{i j}$ is between -1 (completely anti-correlated) and +1 (completely correlated). For the elements on the diagonal $\rho_{i i}=1$. Positive correlation is caused by effects which change the overall number of events (e.g. the magnitude of the neutrino flux): in this case the increase of events in bin $i$ will correspond to an increase of events in bin $j$. Negative correlation is instead caused by effects which change the shape of the distribution and keep the number of events constant: in this case the increase of events in bin $i$ corresponds to a decrease of events in bin $j$ and vice-versa.

The fractional covariance matrix is defined as:

$$
F_{i j}=\frac{E_{i j}}{N_{i}^{C V} N_{j}^{C V}}
$$

The systematic uncertainty for each bin, shown in the plots of Section 9.2, corresponds to the square root of the diagonal elements of the covariance matrix, the fractional error on each bin is given by the square root of the diagonal elements of the fractional covariance matrix, i.e. $\sigma_{i} / N_{i}^{C V}$.

Note that by construction, the covariance, correlation and fractional covariance matrix are symmetrical. These matrices are calculated for the previously introduced selections by the SBNfit framework [103]. 


\subsubsection{BNB flux prediction}

Flux uncertainties are taken from the MiniBooNE BNB flux simulation and adapted to the location and dimensions of MicroBooNE [44]. Three main sources of uncertainty are identified:

- Operational uncertainties related to the horn current.

- Uncertainties on the cross section of the protons and pions with the beryllium target and aluminium-alloy horn.

- Uncertainties related to secondary hadron particles: $\pi^{ \pm}, K^{ \pm}, K^{0}$.

The multisim technique is used to estimate flux uncertainties. The various sources of uncertainties are taken as independent and are varied individually around the central value. They are listed in Table D.1, along the number of universes considered for each. The total uncertainty on the $v_{e}$ flux is found to be $\mathcal{O}(10 \%)$.

Figure 9.1 shows the flux correlation for $v_{\mu}$ (bottom-left) and $v_{e}$ (top-right) interactions. The red bins show large correlation, blue bins indicate anti-correlation. The top-left or bottomright quadrants show the strength of correlations between the two flavors. Correlations are strongest between $v_{e}$ and $v_{\mu}$ fluxes at energies below $1 \mathrm{GeV}$. Therefore, the study of contained low-energy $v_{\mu}$ CC interactions in Section 7.4 can lead to a reduction of modelling uncertainties for the $v_{e}$ flux.

\subsubsection{Cross-section modelling}

Cross-section uncertainties are large due to the scarcity of $v$-Ar cross-section measurements, especially at low energy, $\mathcal{O}(\lesssim 1 \mathrm{GeV})$. Additional uncertainty arises from the complexity of heavy targets such as argon, giving rise to a wide variety of nuclear processes, as described in Section 2.5.

Figure 9.2 shows different cross-section predictions for $v_{\mu}$ and $v_{e}$ charged-current interactions. The different lines correspond to different models used inside the MicroBooNE collaboration. The blue line is the current GENIE central value tune - v3 MicroBooNE- and is 


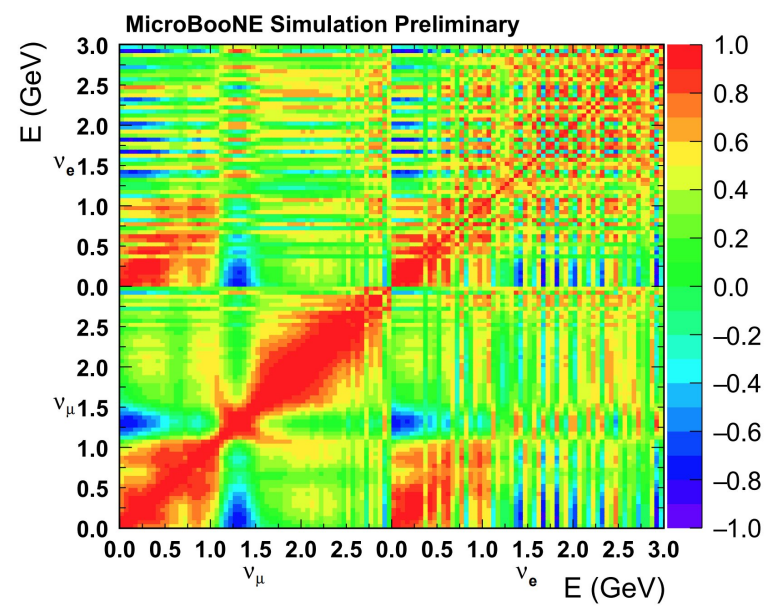

Figure 9.1: $v_{\mu}-v_{e}$ BNB flux correlation matrix. Figure from [44].

guided by fits to the T2K data which increase the CCQE axial mass and scale up the MEC normalisation [42]. The orange line is the default GENIE v3 configuration. The green line represents the GENIE v2 tune used previously by MicroBooNE $[88,89]$. Below $\mathcal{O}(600 \mathrm{MeV})$, the difference in event rates for different models becomes larger than $50 \%$. The large differences between these curves, particularly at low energy, indicate the strong need to constrain cross-section uncertainties with MicroBooNE's own data.

$v$ interaction uncertainties are handled within the GENIE re-weighting package, with the standard re-weighting parameters, also known as "knobs", to model neutrino cross section and FSI uncertainties [58]. A total of 55 sources of uncertainties are considered. They are listed in Tables D.2 and D.3.

\subsubsection{Detector systematic uncertainties}

Detector effects with significant impact on the analysis can be broken up into three main categories:

- Scintillation light modelling

- Wire-Response modelling

- Space-Charge modelling 

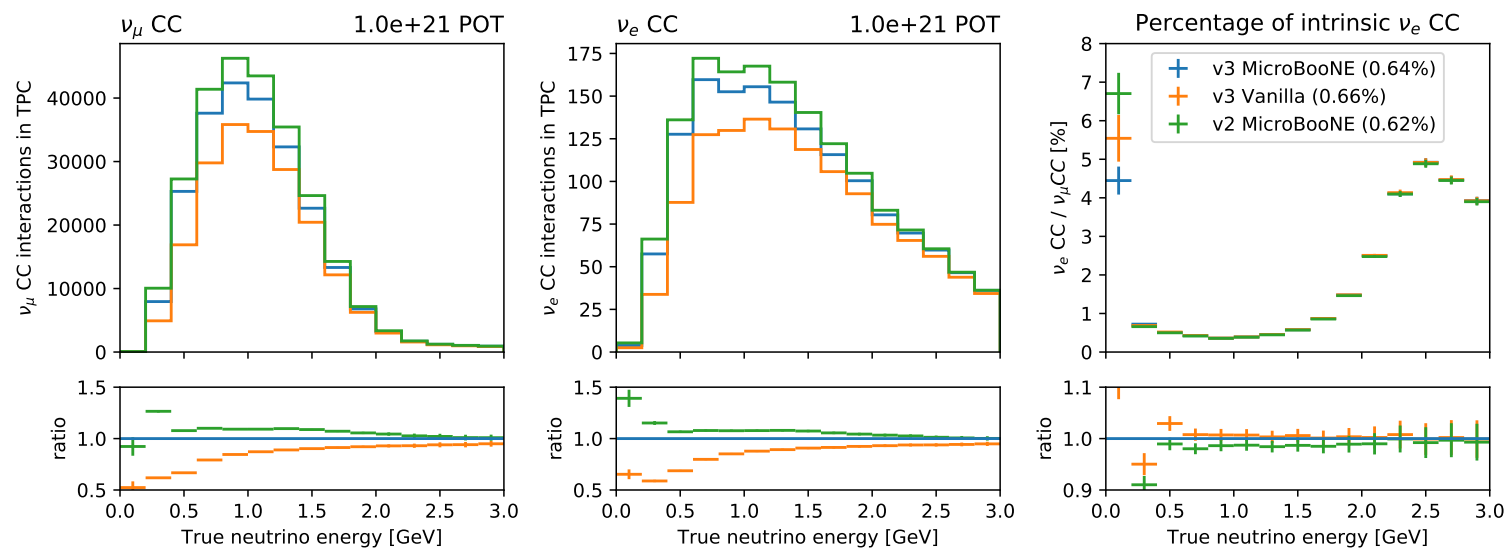

Figure 9.2: Muon and electron charge current interaction rates using different GENIE models. The event rate is scaled to the full MicroBooNE data-set acquired over run period 1-4. (Left) The rates for $v_{\mu}$ CC interactions. (Middle) The rates for $v_{e}$ CC interactions. (Right) The ratio between the charged-current interactions of the different flavours. The legend gives the energy-integrated fraction of $v_{e} \mathrm{CC}$ event to $v_{\mu} \mathrm{CC}$ events. It can be appreciated that the ratio between electron and neutrino interactions is less affected by different interaction models. This is an additional motivation to constrain cross-section uncertainties with MicroBooNE's own data.

Detector systematics samples are generated as unisim variations, meaning that, for each detector effect, a given MC event is re-simulated once with a change to a detector modelling parameter. This produces a new simulation of the same underlying neutrino interactions, which can be used to measure the impact of the detector effect on efficiencies, reconstructed variables and selection rates.

Detector systematics unisims can be used to calculate a covariance matrix for selected event rates in each bin, see Equation (9.1). This covariance is then added to the total covariance matrix. Although computationally favourable, the main drawback of this approach is the fact that the excursion in the number of measured events in a bin computed from a single variation is taken as the $1 \sigma$ excursion for the error estimation. This corresponds to sampling the distribution of variations induced by the detector effect with one draw, which can either over- or underestimate the effect.

In the next sections, comparisons of distributions relevant to the analysis under different 
detector variation samples are shown. However, due to the limited sample availability at the time of writing the thesis, the full inclusion of detector systematics in the analysis has not yet been performed. Some clear differences, such as shifts in energy scale - for example in Figure 9.4a - are visible, though contained to within a few percent. These initial comparisons are promising and suggest a relatively minor impact to the analysis from detector systematics.

Scintillation light modelling MicroBooNE's model of scintillation light production has known limitations. Of particular importance to this analysis, which aims to use a data-set spanning four years, is the significant time-dependent variation of MicroBooNE's light response (see Figure 3.12). This analysis takes several steps to correct for, and mitigate the impact of light decrease that is not modelled in the simulation [63]. However, cosmic rejection and optical triggering on low-energy $v_{e} \mathrm{CC}$ events can be sensitive to scintillation light detector effects.

Two light response variations to estimate the light yield simulation uncertainty are currently available:

$-25 \%$ uniform drop in light yield to account for mis-modelling of the absolute light yield in the detector.

- $120 \mathrm{~cm}$ Rayleigh scattering length, compared to the simulation's default of $60 \mathrm{~cm}$.

Figure 9.3 shows the impact of the earlier described variations on the total amount of light observed in the TPC. Although the variations at low level observables are large, the effect on the selection is restricted to the NeutrinoID (Chapter 6). The NeutrinoID is used to isolate and identify neutrino interactions in each event, relies on scintillation light to identify interactions in-time with the beam and reject cosmic backgrounds. The developed tools reject clusters of TPC activity that are incompatible with the in-time optical flash. This allows the NeutrinoID to rely on light information in a conservative manner and to be impacted minimally by the significant light mis-modelling and time-dependence in scintillation light. 


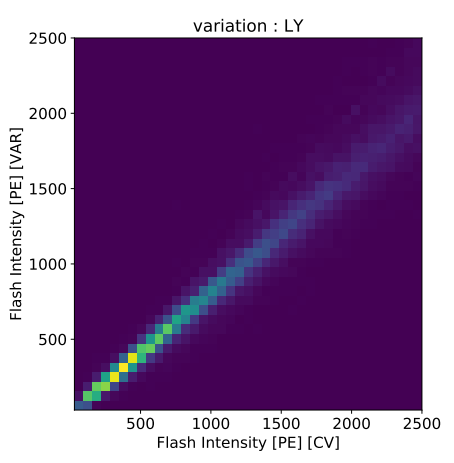

(a) $25 \%$ light yield drop

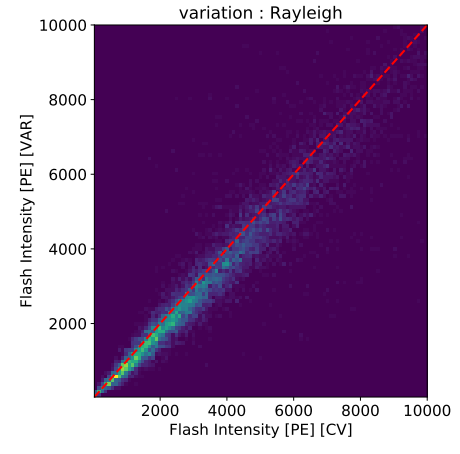

(b) Rayleigh at low $x$

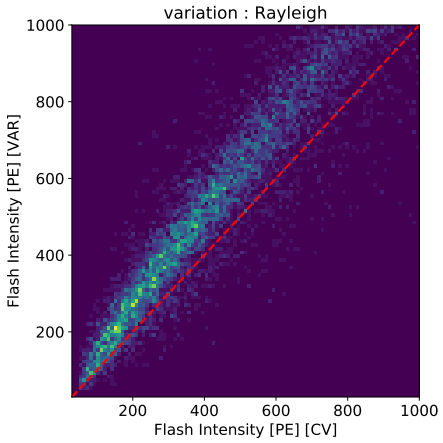

(c) Rayleigh at high $x$

Figure 9.3: Variation in total flash PE recorded for an overall $25 \%$ quenching of light (left) and the impact of an increased Rayleigh scattering length for interactions at low (center) and high (right) $x$, near and far from the PMT rack respectively.

Wire-response modelling The response of MicroBooNE's electronics to drifting charge is a complex subject described in three MicroBooNE publications [57, 72, 71], Section 4.2.2 and Appendix A. The work described in these publications improved the understanding and modelling of noise and field-response effects and has been implemented in the current detector simulation.

The wire modification variations account for changes in wire-response at the local level. Individual waveforms are varied in the $x, y-z, \theta_{X Z}, \theta_{Y Z}$ and $\mathrm{d} E / \mathrm{d} x$ variables. These detector variations are constructed by profiling reconstructed hit variables - charge, amplitude and width - as a function of the mentioned variables with data-driven samples. The hit charge, amplitude and width are then re-scaled in the simulation by the difference between data and simulation. By doing this, the samples cover the difference between data and simulation.

Space charge modelling A data-driven electric-field map is implemented to take into account the electric field distortions, see [104] and Appendix B. Due to the large position distortions, the space charge effect can significantly impact an analysis through the determination of fiducial boundaries, tracking and range-based momentum resolution.

The impact of the wire-response and space charge modelling variation on the $d E / d x$ and 
the track particle log likelihood PID - Section 7.3.1 - are shown in Figures 9.4 and 9.5 respectively. For the latter, the observed smearing introduced by the variations is of the percent level and minimally impacts the clear separation between proton-like (low response) and muon track-like (high response) particles.

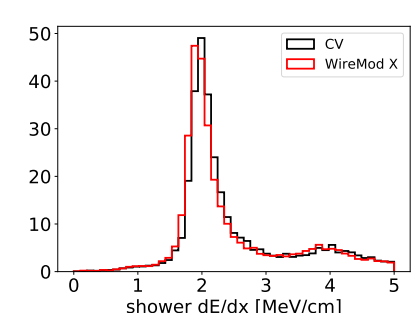

(a) Wire modification $x$

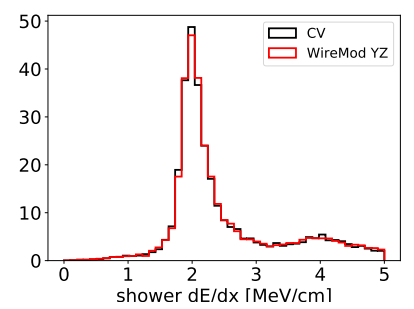

(b) Wire modification $y-z$

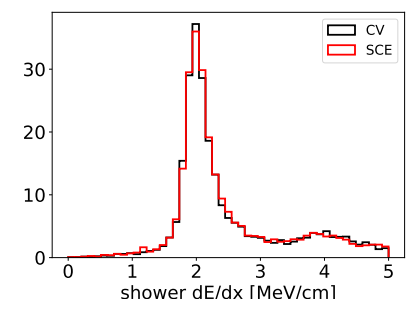

(c) Space charge effect variation

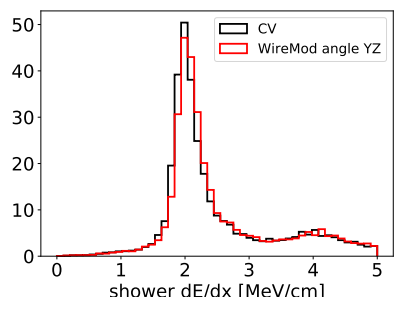

(d) Wire modification $\theta_{Y Z}$

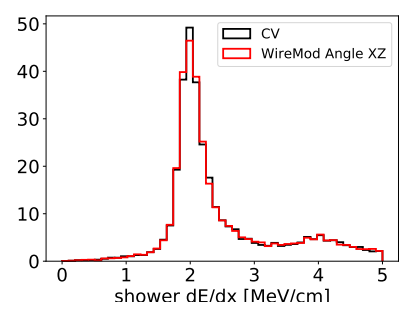

(e) Wire modification $\theta_{X Z}$

Figure 9.4: Impact of TPC waveform variations $(\mathrm{a}, \mathrm{b}, \mathrm{d}, \mathrm{e})$ and the space charge effect variations (c) on the measured electron $\mathrm{d} E / \mathrm{d} x$ after the $v_{e}$ CC preselection.

Recombination of ionisation electrons Recombination accounts for an uncertainty on the conversion from deposited charge to energy, the $\mathrm{d} Q / \mathrm{d} x \rightarrow \mathrm{d} E / \mathrm{d} x$ response of the detector. These samples are currently being developed and are not included in this work. While recombination can impact the total energy resolution, it is expected to be especially important in the particle identification tools developed in Sections 7.3 and 8.3 for muons and electrons respectively. These tools largely depend on the differences in local ionisation density to measure $d E / d x$ accurately. Since recombination can lead to non-linear effects, this systematic uncertainties will be essential in the final analysis. 


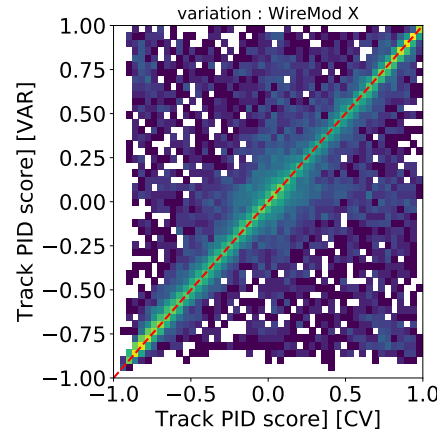

(a) Wire modification $x$

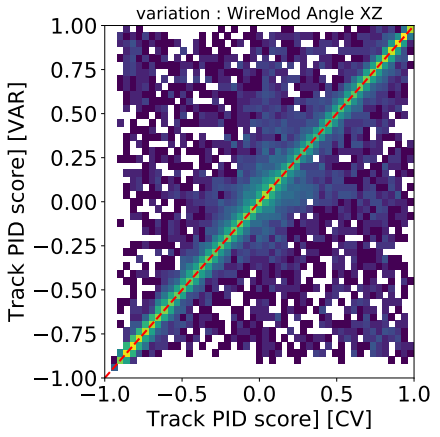

(b) Wire modification $\theta_{Y Z}$

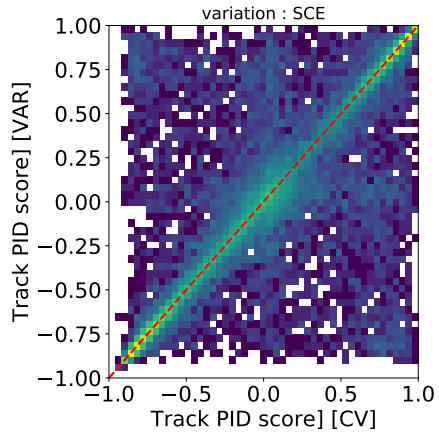

(c) Space charge effect variation

Figure 9.5: Variation in track log likelihood PID, for select TPC response variations. The color scale is logarithmic. 


\subsection{Constraining the systematic uncertainties}

Using the measured muon kinematics in the $v_{\mu} \mathrm{CC}$ channel to constrain the systematic uncertainties of electron kinematics in the $v_{e}$ CC channel is an approach used before in the accelerator-based neutrino oscillation measurements performed by NOvA [105]. This data-driven constraint takes advantage of the high statistics $v_{\mu}$ selection and modelled correlations between electron and muon neutrino fluxes and cross sections. The power of the constraint relies on the fact that the $v_{e}$ and $v_{\mu}$ intrinsic to the beam are both produced by the decay of the same parent $\pi$ and $K$ flux. Similarly, we rely on the charged-current interaction mode, $v_{l}+A r \rightarrow l+X$, common to both $v_{\mu}$ and $v_{e}$ interactions to constrain the uncertainties on the $v_{e}$ interaction modelling.

The richness of $v$-Ar interactions and of hadronic interactions in the beamline offers a number of different handles to constrain different uncertainties using the measurements of $v_{\mu}$ interactions. For example, measurements of CC and NC $\pi^{0}$ production can be used to constrain resonant interactions and thus $\pi^{0}$ backgrounds to the $v_{e}$ selection. Measurements of high-energy $v_{\mu}$ interactions can help constrain the kaon flux in the beam, which contributes substantially to the production of intrinsic $v_{e}$ 's (see Figure 3.4). Likewise, measurements of low-energy $v_{\mu}$ 's can help constrain poorly understood $v$-Ar interaction models in the below $\mathcal{O}(1 \mathrm{GeV})$ energy regime, which is a critical requirement to investigate the MiniBooNE LEE. In this work, the effect of the constraint is demonstrated using the lepton kinematics measurement of contained $v_{\mu} \mathrm{CC}$ interactions as obtained in Chapter 7.

It should be noted, however, that the neutrino identification work described in Chapter 6 results in a highly efficient and topology agnostic selection. A flexible selection, like this one, allows for a number of $v_{\mu}$ measurements and their associated constraints to be implemented in the future if the systematic uncertainties of the $v_{e}$ analysis dominate over the statistical fluctuations and therefore need to be more strongly constrained. Some examples of such exclusive channels were shown in Section 7.4.3.

The procedures described in [106] to include the $v_{\mu}$ CC constraint to the covariance matrix 
are followed. The constraint is performed including the flux+GENIE covariance matrices. In the future, also the covariance matrix arising from detector related systematic uncertainties will be included. It is expected that - although electron shower formation and muon track reconstruction are different - a large portion of the detector effects affect both channels in a similar way and can be constrained.

Here, for this thesis, instead of using the measured data in the $v_{\mu}$ CC lepton kinematics, the Asimov data-set is used [92]. This corresponds to the MC central value, scaled to the total MicroBooNE data-set of $1.01 \times 10^{21}$ POT. When more data is unblinded, the procedure will be repeated using the actual observed data.

The constraint procedure effectively pulls the flux+GENIE universes to the $v_{\mu}$ CC central value, or - in a final stage - to the $v_{\mu}$ CC observed data. Therefore, given the correlation between the $v_{\mu} \mathrm{CC}$ and $v_{e} \mathrm{CC}$ lepton kinematics, this also pulls the flux+GENIE variations in $v_{e}$ CC sample to be closer to the central value. The reduced differences between the central value and the flux+GENIE universes in the $v_{e}$ CC observables lead to constrained systematic uncertainties. The mathematical construction supporting the procedure is described in [107].

The constraint is performed pairwise in the lepton kinematics of the muon and the electron; the muon momentum is used to constrain the electron energy, and the muon $\theta, \phi$ constrain the corresponding electron angles. The $v_{\mu}$ CC muon kinematics are shown in Figure 9.6. Note that the strength of the constraint is based on the accuracy of the reconstruction of the muon and electron kinematic variables. With the current event reconstruction and calibration performances, the resolution on these variables is roughly a factor four better than the reconstructed neutrino energy resolution. A logical next step is to use the combination of the momentum/energy and the $\theta$ angle to constrain the CCQE-like energy as defined in by Equation (2.22).

From Figures 9.6b and 9.8b, it can be seen that the flux+GENIE combined systematic uncertainties before the constraint range between $25 \%$ and $40 \%$. Figure 9.7 illustrates the 
corresponding properties for the $v_{e} \mathrm{CC}$ electron variables. The resolution of the electron energy, which is purely based on the deposited charge on the collection plane is approximately $30 \%$ at the very lowest energies and $20 \%$ at higher energies. The most probably value shows no bias, however, due to the long tail of the distribution, the median has a bias of approximately $10 \%$. This bias stays fairly constant over all energies. The angular distributions do not have a significant bias and the resolution for the $\theta$ and $\phi$ angular variables are $3 \%$ and $5 \%$ respectively.

Figures 9.7b and 9.7c shows the effects of the $v_{\mu} \mathrm{CC}$ constraint on the flux+GENIE systematic uncertainties of the $v_{e}$ CC lepton kinematics. The uncertainties are drastically reduced by a factor three or more in almost the full phase-space. This effect can also be seen by looking at the corresponding fractional covariance matrices before and after the constrain given in Figures 9.8b and 9.8c. Note that by construction of the Asimov data-set and the method the constraint is performed, the covariances in the muon channels vanish since the observed values become the new central values. The main reason - besides the good reconstruction resolution of the lepton kinematics - for the constraining strength is the high truth-level cross-channel correlation, shown in Figure 9.8a.

The number of observed events in Figure 9.7c are scaled to $8.6 \times 10^{18}$ POT. The full Run 1-4 data-set is 117 times bigger. Therefore, the expected number of events - maintaining the chosen binning - will range from 10 to 150 events per bin, leading to statistical fluctuations of $8 \%$ to $30 \%$. This demonstrates the importance of the data-driven $v_{\mu} \mathrm{CC}$ constraint to keep the $v_{e}$ CC result statistics limited. 

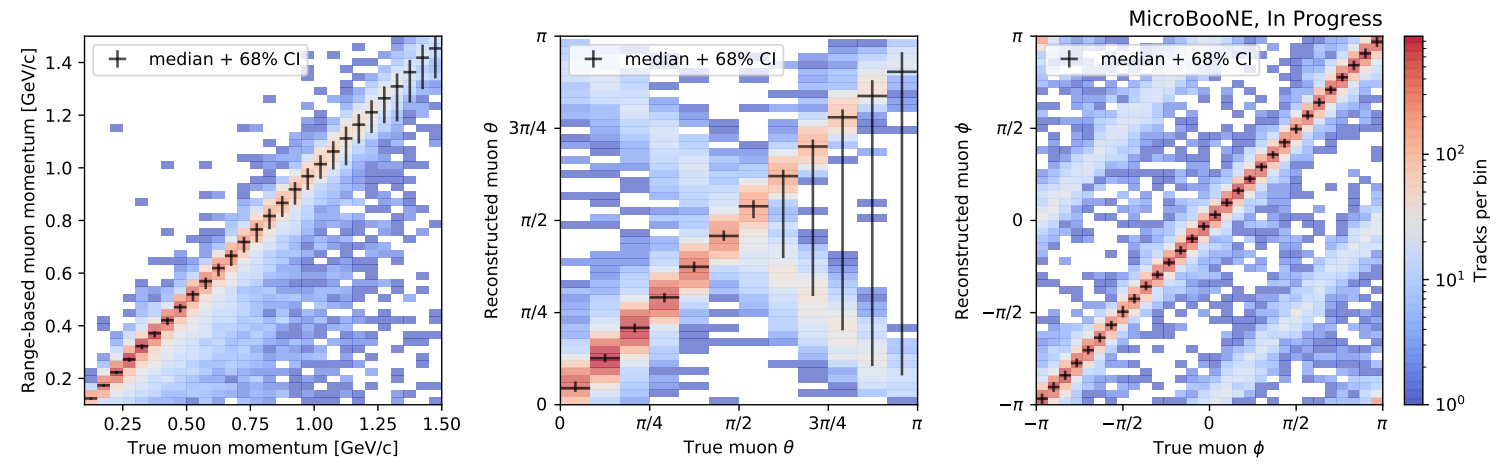

(a) Reconstructed resolution in the contained $v_{\mu}$ CC selection for the muon momentum (left), muon $\theta$ (middle) and muon $\phi$ (right). The colour scale for the $2 D$ histograms is logarithmic. In black, the median and $68 \%$ confidence interval are given, binned in the true muon kinematics. For all three muon kinematics, the most probable value has as bias at the $\mathcal{O}(1 \%)$ level. The muon momentum resolution is equivalent to the track length reconstruction resolution. For short tracks, this is below $3 \%$, for longer tracks, that have a higher probability to enter unresponsive regions of the detector, this increases to $\mathcal{O}(5 \%)$. The middle and right plot show off-diagonal components related to reverse reconstructed muon tracks. When excluding these spurious cases the resolution in $\theta$ is $\mathcal{O}(2 \%)$ and $\mathcal{O}(3 \%)$ for $\phi$.
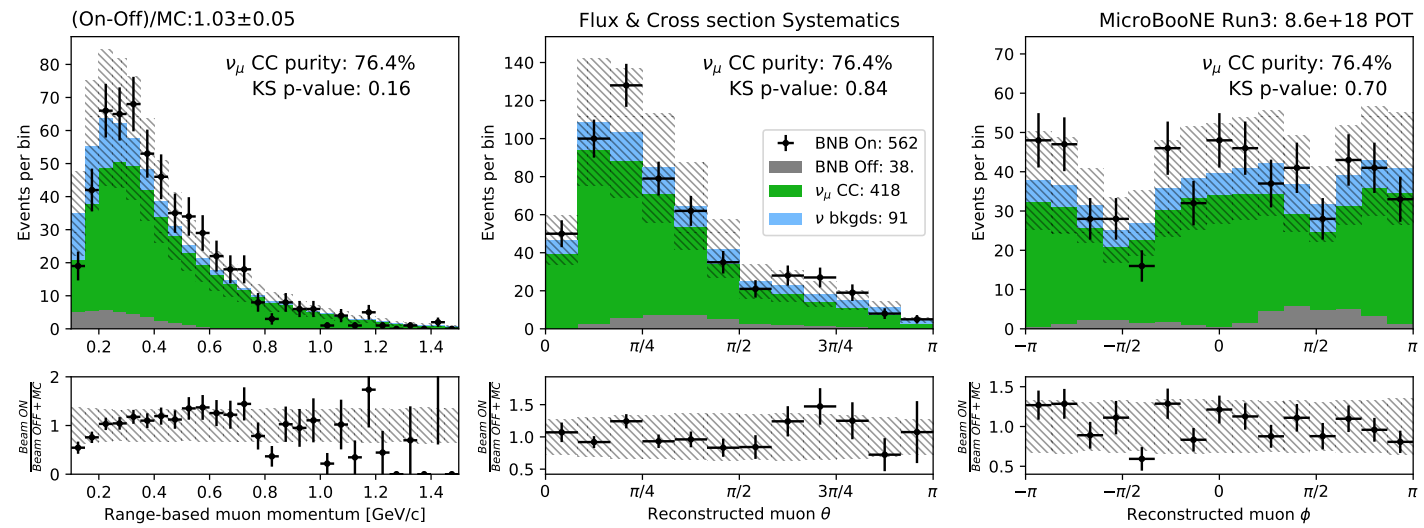

(b) Momentum (left) and directional angles $\theta$ (middle) and $\phi$ (right) of the $v_{\mu}$ CC muon candidate. In blue, the neutrino related backgrounds are grouped into one category. The error bars on the data are Poissonian. The errors on the prediction are the flux+GENIE combined systematic uncertainties as described in Sections 9.1.1 and 9.1.2. The fractional magnitude of the systematic uncertainties ranges between $25 \%$ and $40 \%$.

Figure 9.6: Lepton kinematics for the $v_{\mu} \mathrm{CC}$ channel. Both resolution (a) and data/MC comparisons (b) are shown. 

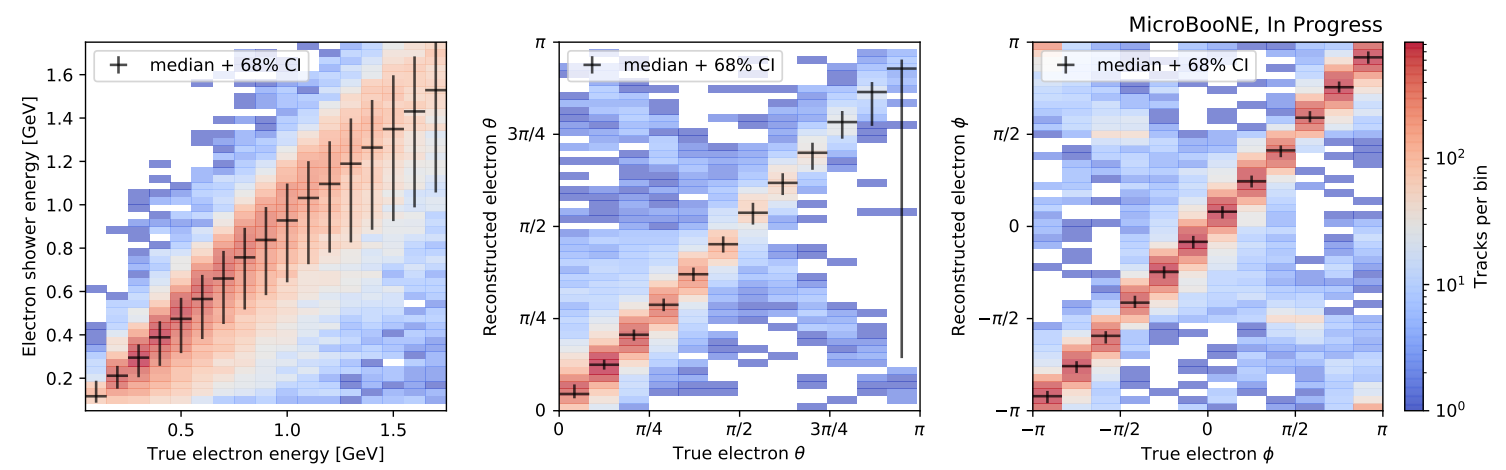

(a) Resolution for the electron variables after the $v_{e}$ CC selection. The colour scale is logarithmic. In black, the median and $68 \%$ confidence interval are given, binned in the true electron kinematics.
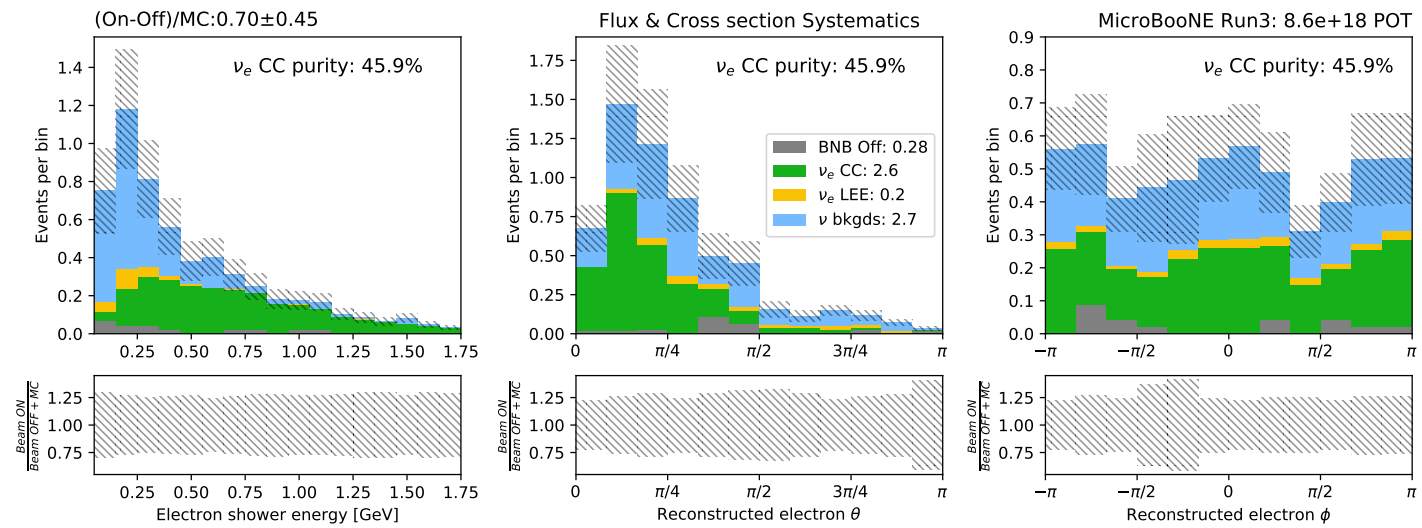

(b) Electron shower energy (left) and directional angles $\theta$ (middle) and $\phi$ (right) of the $v_{e}$ CC electron candidate. In blue, the neutrino related backgrounds are grouped into one category. The error bars on the data are Poissonian. The errors on the prediction are the flux+GENIE combined systematic uncertainties before constraint. The fractional magnitude of the systematic uncertainties ranges between $25 \%$ to $40 \%$.
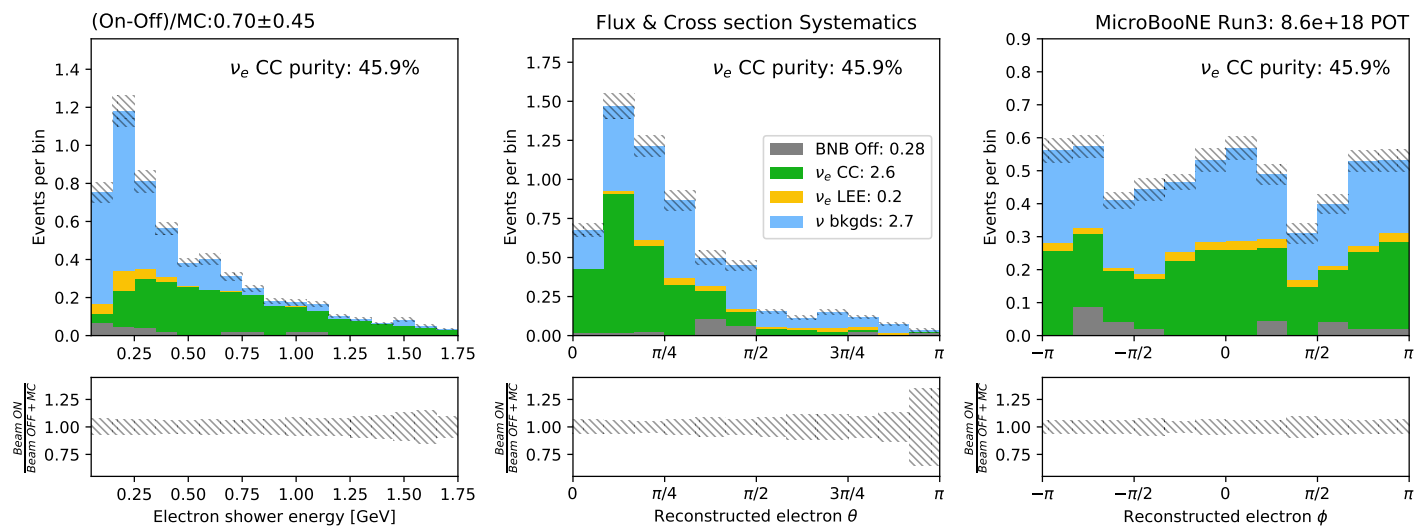

(c) Analogous to (b). The systematic uncertainties after constraint are now shown. The size of the systematic uncertainties is considerably smaller and ranges between $5 \%$ to $15 \%$.

Figure 9.7: $v_{e}$ CC electron kinematics. Both the resolution (a) and parameter distributions (b) are shown. The four selected data events in the Run3 $9 \times 10^{19}$ POT are omitted for clarity. 

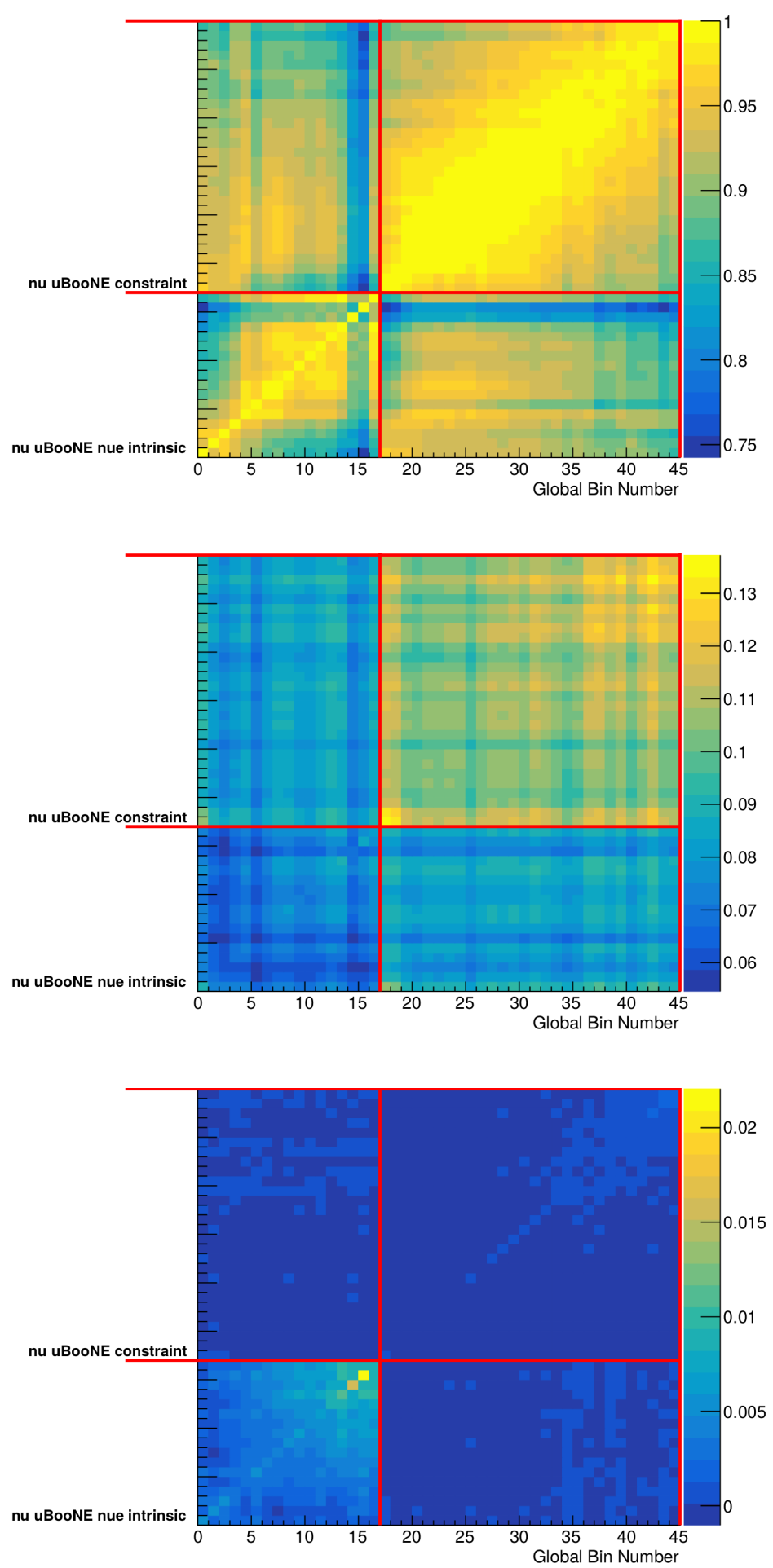

(a) Correlation matrix between the two selections. The correlation both within and between the channels is very high, emphasising the power to constrain each other.

(b) The fractional covariance matrix before the constraint. The diagonal elements correspond to the square of the fractional error in that bin and are of the order of $30 \%$.

(c) The fractional covariance matrix after the constraint. By construction of the constraint using the $v_{\mu}$ CC Asimov data-set, the covariance disappears in the $v_{\mu} \mathrm{CC}$ channels. The fractional errors in the $v_{e} \mathrm{CC}$ channels is greatly reduced and are of the order of $10 \%$.

Figure 9.8: Covariance and derived matrices produced by SBNFit to perform the data-driven constraint of the $v_{e} \mathrm{CC}$ flux+GENIE systematic uncertainties with the $v_{\mu} \mathrm{CC}$ selection. The bottom-left sub-matrix represent the electron shower energy part and the top-right contains the muon momentum bins. The off-diagonal elements connect both channels. 


\subsection{Electron neutrino measurement \& sensitivity to new physics}

In this section the $v_{e} \mathrm{CC}$ selection as obtained in Chapter 8 and shown in the bottom-right panel of Figure 8.22 will be used to gauge the potential to characterise the intrinsic $v_{e}$ component in the BNB and observe new physics. Furthermore, the sensitivity to new physics will be studied by testing a $3+1$ sterile neutrino model.

Due to the limited availability of the samples to include systematic uncertainties, the procedures described here account for statistical fluctuations only. However, as demonstrated previously in Section 9.2, after constraining the systematic uncertainties with a $v_{\mu}$ CC dataset, one expects their magnitude to be of the same order of importance as the statistical fluctuations for the full MicroBooNE data-set of $1.01 \times 10^{21}$ POT. For any smaller data-set, the result is expected to be statistically limited. The inclusion of systematic uncertainties on the results obtained in this section can be approximated as a widening of the uncertainties and, therefore, will be of limited impact on the narrative of the following paragraphs.

For any measurement or sensitivity study, an observable needs to be chosen. While technically the number of events works, including the shape of the distribution enhances the power of the measurement. This becomes clear from Figure 9.7c, where the $v_{e}$ LEE signal, the $v_{e} \mathrm{CC}$ intrinsic and the background distributions have different shapes. For clarity and simplicity, the reconstructed electron shower energy is chosen as the observable used in this section. However, it is expected that a combination of the electron energy and shower direction - such as the CCQE-like energy (Equation (2.22)) - will increase the significance of the results. This is true if the underlying physics causing a hypothetical anomaly is dependent on the neutrino energy rather than the lepton kinematics, which is the case for a sterile neutrino oscillation.

\subsubsection{Statistical-only likelihood construction and sensitivity estimation}

The basic ingredient of statistical inference is the likelihood. We parametrise the expected distribution in terms of the signal strength $\mu$. This is a scale factor of the expected signal. In the case of the measurement of the electron neutrinos in the beam, $\mu=1$ corresponds to the 
expected number of electron neutrinos, whereas $\mu=0$ corresponds to no electron neutrinos in the beam. Any other value of $\mu$ indicates an anomalous number of electron neutrinos. Therefore, the expected number of events observe in a bin is:

$$
b_{i}+\mu \cdot s_{i}
$$

Here, $i$ is the bin index, $s$ and $b$ are the predicted events corresponding to the signal and background model respectively. The probability, therefore, to observe an actual number of $n_{i}$ events in the bin is assumed to be Poisson distributed:

$$
P\left(n_{i} \mid \mu\right)=P_{\text {Poisson }}\left(n_{i} \mid b_{i}+\mu s_{i}\right)=\frac{\left(b_{i}+\mu s_{i}\right)^{n_{i}} e^{-b_{i}-\mu s_{i}}}{n_{i} !}
$$

The likelihood to observe a certain distribution, assuming the observation in each bin is independent, becomes:

$$
\mathcal{L}(\mu)=\prod_{i=1}^{N} P\left(n_{i} \mid \mu\right)
$$

For computational reasons, instead of maximising the likelihood, it is preferable the minimise the negative log likelihood:

$$
-\log (\mathcal{L}(\mu))=-\sum_{i=1}^{N} \log \left(P\left(n_{i} \mid \mu\right)\right) .
$$

Plugging in the Poisson distribution, Equation (9.6), and dropping the factorial term in the denominator that is independent of $\mu$, we obtain:

$$
-\log (\mathcal{L}(\mu))=-\sum_{i=1}^{N}\left(n_{i} \log \left(b_{i}+\mu s_{i}\right)-b_{i}-\mu s_{i}\right)
$$

In the next paragraphs, the likelihood will be evaluated using the Asimov data-set for special values of $\mu$ :

- $\mu=0$, the $H_{0}$ or null hypothesis, i.e. only background events are observed: $n_{i}=b_{i}$.

- $\mu=1$, the $H_{1}$ hypothesis, i.e. the observation coincides with the signal model: $n_{i}=$ $b_{i}+s_{i}$. 
Note that the likelihood can be evaluated for non-integer values of $n_{i}$.

In the approximation of many events per bin $n_{i} \gtrsim 10$, the central limit theorem states that the likelihood becomes approximately Gaussian in function of $\mu$ around its maximum. If we define $\mu_{0}$ as the value for which the likelihood is maximal,

$$
\mathcal{L}(\mu) \approx \mathcal{L}\left(\mu_{0}\right) e^{-\frac{1}{2}\left(\frac{\mu-\mu_{0}}{\sigma}\right)^{2}}
$$

The difference in the log likelihood around the maximum value at $\mu=\mu_{0}$ is:

$$
-\left.\Delta \log (\mathcal{L}(\mu))\right|_{\mu_{0}}=-\log \left(\frac{\mathcal{L}(\mu)}{\mathcal{L}\left(\mu_{0}\right)}\right) \approx \frac{1}{2}\left(\frac{\mu-\mu_{0}}{\sigma}\right)^{2}
$$

where it is assumed that $\sigma$ is constant in the region around $\mu_{0}$ in which $\mu$ is varied. One can now obtain the difference in the log likelihood corresponding to $k$ units of the standard deviation by taking $\mu=\mu_{0}+k \sigma$ :

$$
-\Delta \log (\mathcal{L}(k)) \approx \frac{k^{2}}{2}
$$

Therefore - in the Gaussian approximation - the one $\sigma$ interval is determined by $-\Delta \log (\mathcal{L})=$ $1 / 2$, the two $\sigma$ interval by $-\Delta \log (\mathcal{L})=2$ and so forth.

Statistical hypothesis testing The prescription above suffices to determine the signal strength from the maximum likelihood fit and its corresponding statistical-only uncertainty from the shape of the negative log likelihood around the minimum. To determine the sensitivity to a hypothesis $H_{1}$, such as the presence of new physics, we need a prescription to perform a hypothesis test:

- Choice of the test statistic. This is a function of the observables only and condenses all information of the observables into one number.

- Definition of the two hypotheses. $H_{0}$ is the null hypothesis, whereas $H_{1}$ is the hypothesis where we do observe a pre-defined signal model. 
- Toy-experiments or pseudo-experiments. The expected distributions of the test statistic under the two hypotheses are built by randomly sampling the observables from their expected distributions. In case of the statistical-only sensitivities, the bins of the histograms are treated as independent Poisson variables as described above. In case systematic uncertainties are considered, the bins of the histograms are treated as a multi-variate Gaussian distribution, determined by the mean values and by a covariance matrix, which is then convoluted with a Poisson sampling that accounts for the statistical fluctuations.

- Once each toy-experiment is performed, and a distribution for the bin contents of the histograms is observed, the discovery significance, i.e. reject $H_{0}$, can be computed. This is done by computing the value of the test statistic associated to the observation. The $p$-value is obtained from the expected distribution of the test statistic under $H_{0}$. (See the right panel of Figure 9.10 as an example.)

- To compute the expected significance, one can consider the median value, and an interval which contains $68 \%$ or $95 \%$ of the distribution of the test statistic under $H_{1}$, and compute the $p$-values with respect to $H_{0}$.

- The $p$-value is typically converted to the number of sigma of a standard Gaussian that produces the same $p$-value, as outlined in [108].

In contrast to the maximisation of the likelihood to obtain the signal strength, in this hypothesis test prescription, no parameter is extracted from the observed data.

The hypothesis of background-only is tested against the hypothesis of background-plussignal. In this work, the ratio of the likelihoods under the two hypotheses is chosen as a test statistic. In fact, it can be proven to be the most powerful test, yielding the highest significance given the expected observable distribution, a null hypothesis and a signal hypothesis [92]. From Equation (9.9), we obtain:

$$
T_{\mathrm{LLR}}=-\log \left(\frac{\mathcal{L}\left(\mu_{H_{1}}\right)}{\mathcal{L}\left(\mu_{H_{0}}\right)}\right)=\sum_{i=1}^{N}\left(\mu_{H_{1}}^{i}-\mu_{H_{0}}^{i}-n^{i} \log \left(\frac{\mu_{H_{1}}^{i}}{\mu_{H_{0}}^{i}}\right)\right)
$$


where LLR stands for log-likelihood-ratio of the Poisson likelihoods and $\mu_{i}$ is the expected bin content.

\subsubsection{The intrinsic $v_{e}$ BNB component}

As a first step, the background only hypothesis of a pure muon beam content will be assumed. The $H_{1}$ hypothesis corresponds in this scenario to the observation of an electron neutrino contribution in the BNB flux. The sensitivity to "discover" $v_{e}$ 's in the BNB is shown in the left panel of Figure 9.9 in function of the collected data size. The shown sensitivities are obtained using Equation (9.13).

As can be observed from the figure, the current open data-sets $-9 \times 10^{18}$ POT and $4 \times 10^{19}$ POT - provide us with an expected sensitivity to $v_{e}$ observation of approximately $1 \sigma$ and $3 \sigma$ respectively. These estimates are in agreement with the analysis of selected data events, of which some examples were shown in Figure 8.25.

The actual Run 1-4 data-set collected by MicroBooNE is 20 times larger, $1.01 \times 10^{21}$ POT, and will enable us to measure the electron contribution in the BNB. In the right panel of Figure 9.9, the negative log likelihood is plotted in function of $\mu$ using Equation (9.9). For this exercise, the Asimov data-set corresponding to the simulated MicroBooNE intrinsic $v_{e}$ flux and cross-section - as illustrated in Figure 9.2 - is used. From the red line, representing the full data-set, it can be concluded that the developed $v_{e}$ CC selection leads to a statisticalonly measurement of the BNB $v_{e}$ component with a $1 \sigma$ interval corresponding to $\mathcal{O}(10 \%)$.

While "discovering" the intrinsic $v_{e}$ component in the BNB may seem a futile exercise, it is important to stress that currently the MicroBooNE full data-set is still blinded. Therefore, the best metric to assess the strength of this analysis is its sensitivity to the intrinsic $v_{e}$ CC events. It is demonstrated from the left panel in Figure 9.9 that even with the limited data-set, a median statistical-only sensitivity of $\mathcal{O}(4 \sigma)$ can be obtained. Furthermore, the extraction of the signal strength $\mu$ of the intrinsic $v_{e}$ component, in combination with the observed distributions of the three lepton kinematic variables, is a crucial step towards our understanding of electron neutrino modelling and the observation of a potential anomaly 

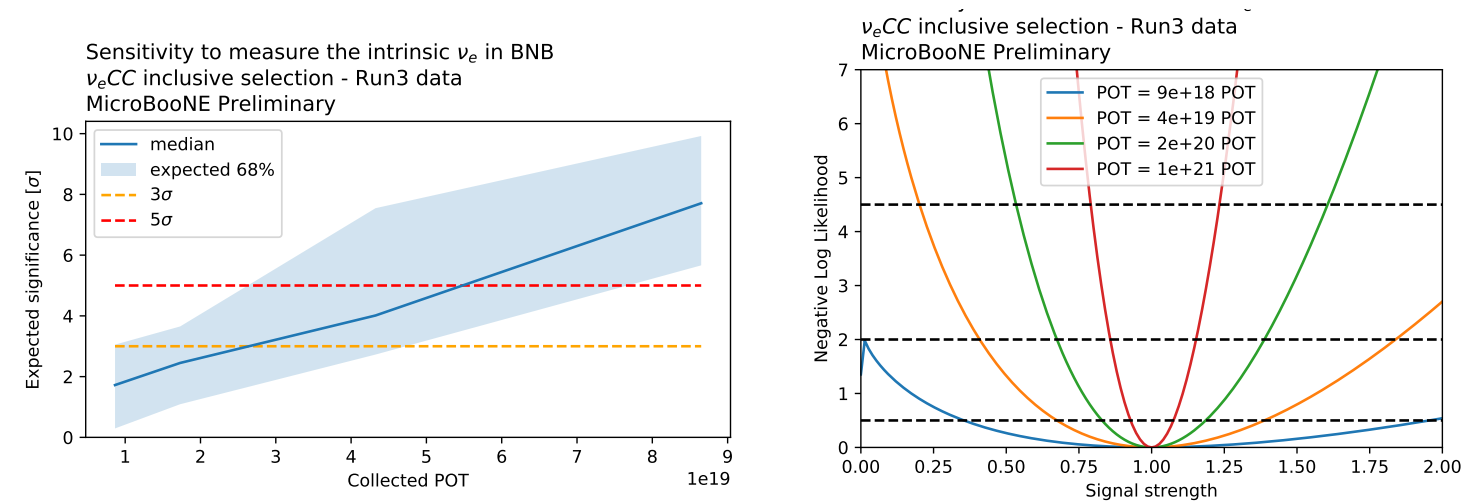

Figure 9.9: (Left) Sensitivity to the observation of $v_{e}$ CC interaction from the BNB flux. (Right) Estimation of the uncertainty on the $v_{e}$ signal strength. The $1 \sigma, 2 \sigma$ and $3 \sigma$ intervals correspond to a $-\Delta \log (\mathcal{L})$ of $1 / 2,2$ and $9 / 2$ respectively. Both results are obtained by using the Asimov data-set corresponding the MicroBooNE simulation. Figures produced in collaboration with Nicolò Foppiani.

in MicroBooNE.

\subsubsection{Sensitivity to a $3+1 v_{S}$ oscillation signal}

In addition to quoting the sensitivity to observe the intrinsic $v_{e}$ contribution in the BNB, the sensitivity to a $3+1 \mathcal{O}(\mathrm{eV})$ sterile-neutrino oscillation signal is studied. The nature of this analysis, and the strong connection of past short-baseline anomalies to sterile neutrino models, makes the investigation of such a hypothesis interesting and relevant. While strong tension exists in global fits to $3+1$ signals, this model is less dependent on MiniBooNE's detector and neutrino interaction modelling compared tot the unfolded MiniBooNE LEE signal. The model under study is a simple extension of the Standard Model with one sterile neutrino as introduced in Section 2.3.1.

The effective oscillation probability $v_{\mu} \rightarrow v_{e}$ is:

$$
P^{\text {osc, short baseline }}\left(v_{\mu} \rightarrow v_{e}\right)=\sin ^{2}\left(2 \theta_{e \mu}\right) \sin ^{2}\left(1.27 \Delta m_{14}^{2} \frac{L}{E}\right)
$$

which is characterised by an effective mixing parameter $\sin ^{2}\left(2 \theta_{e \mu}\right)$ and oscillation frequency $\Delta m_{14}^{2}$. The same framework as introduced in Section 9.3.2 is used to perform a simple 
hypothesis test and compute expected statistical-only sensitivities. This means that no fit or extraction of these parameters is performed at this stage. The parameter values of the best fit to the appearance experiments is chosen, as taken from [109]. The parameters are $\sin ^{2}\left(2 \theta_{e \mu}\right)=0.00697$ and $\Delta m_{14}^{2}=0.573 \mathrm{eV}^{2}$. The contribution from the oscillation to the expected $v_{e}$ CC flux is shown in Figure 9.10. For this specific set of parameters, the signal appears at $E\left(v_{e}\right)$ of $\mathcal{O}(500 \mathrm{MeV})$. At these energies, the $v_{e}$-argon cross section is significantly lower, therefore suppressing the signal interaction rate.

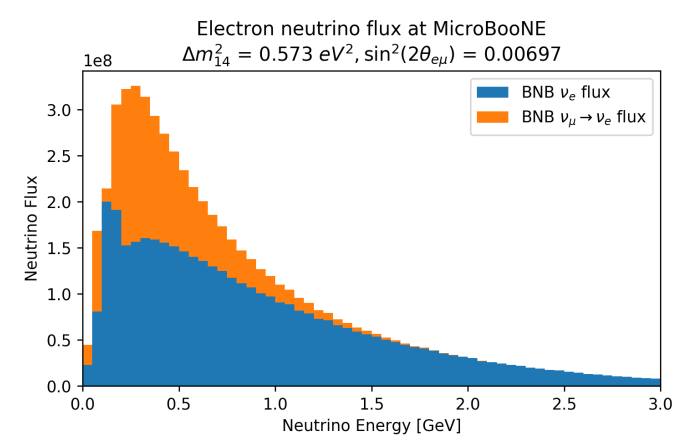

Figure 9.10: An additional sterile neutrino with a mass much larger than the ordinary neutrinos would induce an effective muon neutrino to electron neutrino oscillation probability. The contribution to the intrinsic $v_{e}$ flux are shown for the oscillation parameters values $\sin ^{2}\left(2 \theta_{e \mu}\right)=0.00697$ and $\Delta m_{14}^{2}=0.573 \mathrm{eV}^{2}$. Figure produced in collaboration with Nicolò Foppiani.

The sensitivity is estimated using the $v_{e}$ CC inclusive selection as developed in Chapter 8 . The left plot in Figure 9.11 shows the expected reconstructed shower energy spectrum in the presence of the signal. The blue distribution represents the $H_{0}$ hypothesis and includes both selected $v_{e}$ CC events as well as background events. The individual contributions can be seen in the bottom-right panel of Figure 8.22. The right plot shows the distribution of the Poisson log-likelihood-ratio test-statistic under the null hypothesis (blue) and oscillationsignal hypothesis (orange), with the expected significance to this signal.

For the chosen sterile neutrino parametrisation, the expected median sensitivity of observation, $1.8 \sigma$, is fairly limited due to the low efficiency and large background contribution at low energy, see Figure 8.23. Nevertheless, recently, experiments like IceCube, Neutrino4 
and DANSS have observed indications of sterile neutrinos with slightly higher masses and stronger mixing parameters [110, 111, 112]. These signals would pop up at higher electron neutrino energies in MicroBooNE and would be observed by this analysis.

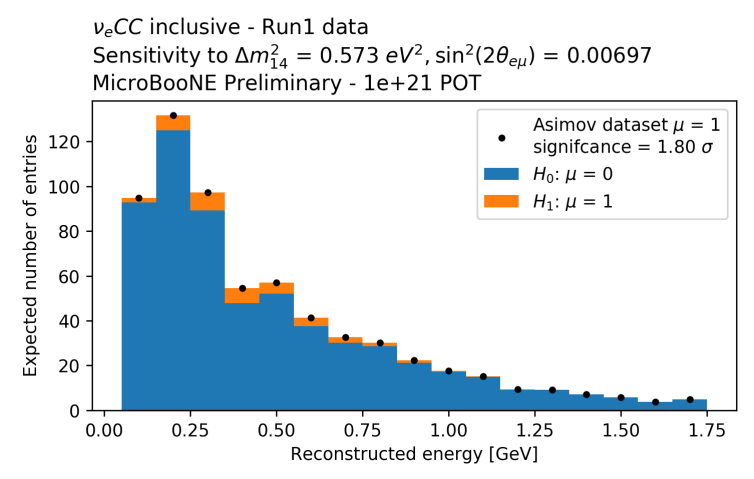

(a) $E_{\text {reco }}$ of the electron candidate shower

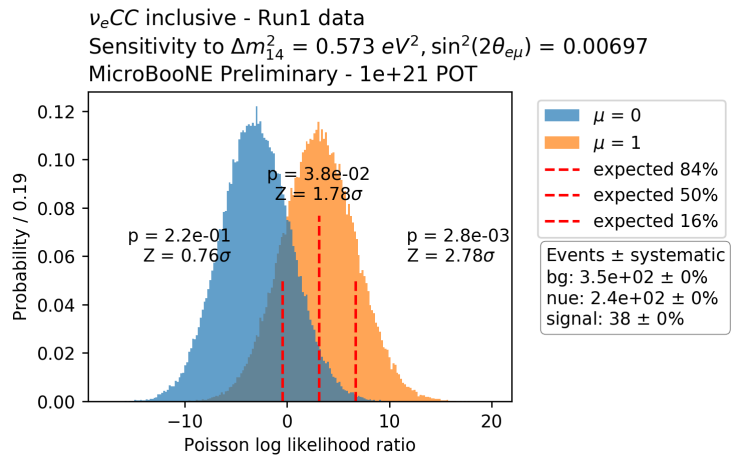

(b) Poisson LLR test statistic distributions

Figure 9.11: (Left) The contribution caused by the presence of a sterile neutrino to the spectrum of the reconstructed electron candidate shower. The contribution is much smaller than the background and $v_{e} \mathrm{CC}$ contribution. This is explained by the low efficiency at low energies $-\mathcal{O}(500 \mathrm{MeV})$ where the signal is located. (Right) The distribution of the test statistic for the null and alternative hypothesis shows a median expected significance around $2 \sigma$ for these values of the oscillation parameters. Figures produced in collaboration with Nicolò Foppiani.

\subsubsection{Sensitivity to the MiniBooNE low-energy excess}

In Section 8.1.3, a signal model was constructed to predict - under a sizeable amount of caveats - how the signal observed by MiniBooNE would look like in MicroBooNE if the underlying cause of the anomaly are electron neutrinos. Completely analogue to Section 9.3.3, the unfolded MiniBooNE LEE signal plus the nominal expected events is taken as $H_{1}$ hypothesis. The obtained sensitivity median sensitivity is $\approx 1.5 \sigma$ and is shown in Figure 9.12. 


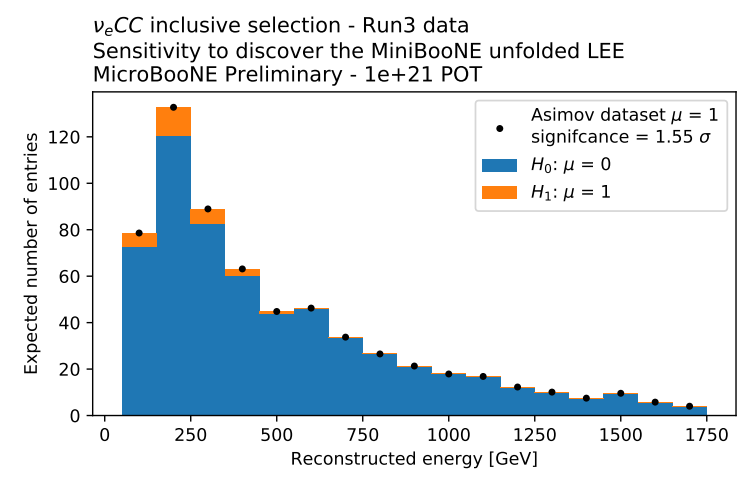

(a) $E_{\text {reco }}$ of the electron candidate shower

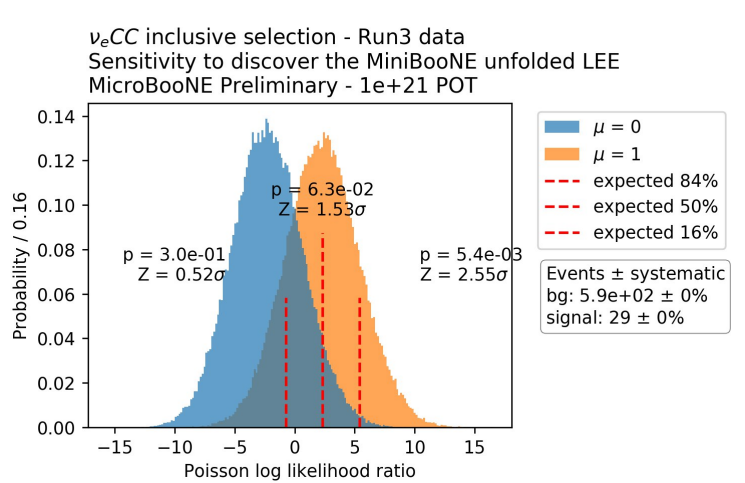

(b) Poisson LLR test statistic distributions

Figure 9.12: (Left) The contribution caused by the LEE signal to the spectrum of the reconstructed electron candidate shower. (Right) The distribution of the test statistic for the null and alternative hypothesis shows a median expected significance around $1.5 \sigma$. Figures produced in collaboration with Nicolò Foppiani. 
"Estragon - On trouve toujours quelque chose, hein, Didi, pour nous donner l'impression d'exister? Vladimir (impatiemment) - Mais oui, mais oui, on est des magiciens."

Samuel Beckett, En attendant Godot

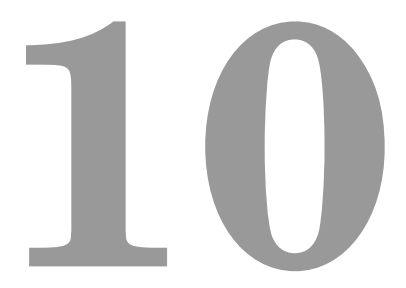

\section{Conclusion \& Outlook}

The MicroBooNE experiment is a crucial step in the understanding of the observed shortbaseline anomalies. Furthermore, being the first LArTPC in a neutrino beam with automated event reconstruction and selection, a wide range of $v$-argon physics can be explored. The work presented in this thesis gave a comprehensive overview of what results can be expected from the collaboration in the next year.

A cosmic-ray measurement was performed. For the first time, the three MicroBooNE subdetector systems were validated while obtaining a physics result: the characterisation and rate of cosmic muons.

The understanding and tools developed to study cosmic activity were further exploited to eliminate cosmic activity and isolate neutrino interaction in the TPC. This flavour agnos- 
tic neutrino selection is now fully incorporated in a wide range of MicroBooNE analyses. Among which is a plethora of cross-section measurements and new physics searches, both on neutrinos from the BNB and NuMI beams.

The neutrino selection was extended with muon identification tools to obtain a $70 \%$ pure charged-current neutrino sample. For the first time, the full MicroBooNE data-set is opened and filtered with this selection. This enables the collaboration to look at $\mathcal{O}\left(1 \times 10^{5}\right) v_{\mu} \mathrm{CC}$ events, and investigate a set of high-statistics cross-section results in exclusive muon neutrino channels, aimed to be released by Summer 2020 .

Chapter 8 focuses on the identification of $v_{e}$ CC events. A machine-leaning-based electron classifier was developed, comprising a set of physics-guided electromagnetic shower features and trained with simulated data. It was demonstrated that an $e-\gamma$ enhancement of a factor 15:1 can be achieved, effectively reducing $\pi^{0}$ related backgrounds with $95 \%$. Furthermore, this supports the claim that MicroBooNE is able to pin down to origin of an excess in electromagnetic events, if observed. A charged-current electron selection with an efficiency of $20 \%$ was shown, together with the distributions of the lepton kinematics. This is the first time that an efficiency this high is combined with a high purity, leading to an expected total of $300 v_{e}$ CC events in the full data-set.

The developed selections are combined in Chapter 9, demonstrating the power of a datadriven constrain of the systematic uncertainties in the electron neutrino kinematic measurement. Additionally, statistical-only procedures are outlined to measure the signal strength of the electron neutrino beam content and investigate the rejection sensitivity concerning new physics models such as the sterile neutrino.

The near future This work paved the path towards a $v_{e} \mathrm{CC}$ measurement with the BNB. However, the finalisation of the data-driven systematic uncertainties constraint procedure and its incorporation in the final measurement is still to be completed. This will be carried out in the next months. In parallel, the opening of the box strategy and intermediate steps are currently being decided by the collaboration. The data shown in the final results of this 
thesis is a fraction - less than $5 \%$ - of the available collected data by the experiment over the past four years, illustrated in Figure 10.1.

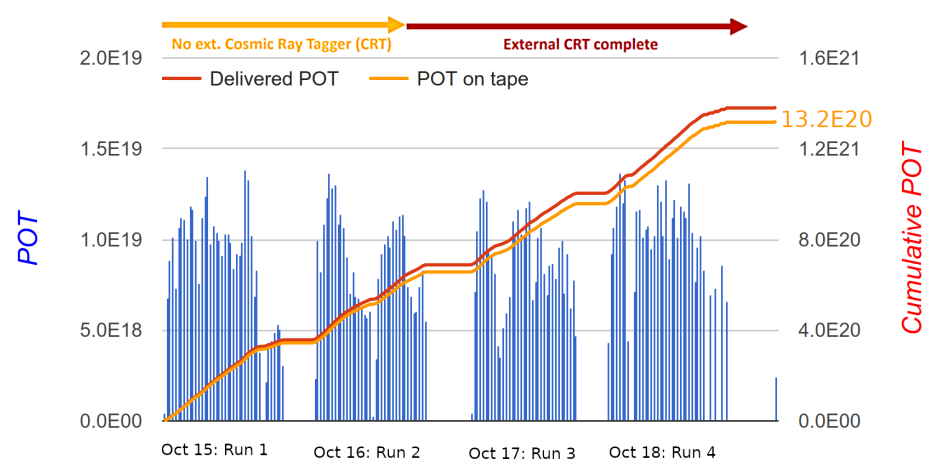

Figure 10.1: MicroBooNE total Protons on Target collected with the Booster Neutrino Beam during the first four run periods. Throughout this thesis, parts of the first three run periods were used.

Although a valuable characterisation of electron neutrinos was performed, the inclusive $v_{e}$ CC selection is not sensitive to the unfolded MiniBooNE low-energy excess at the $3 œ$ level. Therefore, within the collaboration, different approaches are devised. A tailored $v_{e}$ CC Np low-energy selection with an overall efficiency below $10 \%$, but high-purity at the lowest energies was developed simultaneously with the one shown in this work [113]. Together, both selections will inform the community about the electron neutrino interactions observed by MicroBooNE.

The slightly-less-near future It is likely, that even with the combination of multiple selections and data-driven cosntraints, MicroBooNE will not be able to fully resolve the shortbaseline anomaly. However, even if that is the case, the Short-Baseline Neutrino Program at Fermilab, introduced in Section 3.4, will turn on soon and perform an oscillation search. Within the next five years, it aims to cover the phase space of the short-baseline anomalies by studying both $v_{e}$ appearance and $v_{\mu}$ disappearance in the BNB. The progress of SBN, sharing the same technology and beam as MicroBooNE, will benefit hugely from the LArTPC expertise developed by the collaboration, emphasising the relevancy of this thesis. 


\section{A Unresponsive Wires \& Charge Calibration}

Unresponsive wires in the MicroBooNE TPC A study of the status of the TPC readout channels in MicroBooNE was conducted after installation and filling of the cryostat with liquid argon. The pedestal distribution during regular data taking and the ASIC impulse response using calibration data taken with the external pulser are used to identify both operational and non-functioning channels. It was fount that about $10 \%$ of the wire readout channels are nonfunctional for various reasons [57]. Figure A.1 shows the area of the nonfunctional wires on the anode plane where $30 \%$ area is unresponsive. In reality, two active planes can often be sufficient for reasonable event reconstruction [73]. Requiring at least two active wire planes results in a significantly improved volume efficiency about $97 \%$.

Charge Calibration Throughout this thesis, the $\mathrm{d} E / \mathrm{d} x$ of reconstructed particles, alongside with their calorimetric deposited energy, is used. The first step of energy reconstruction in 


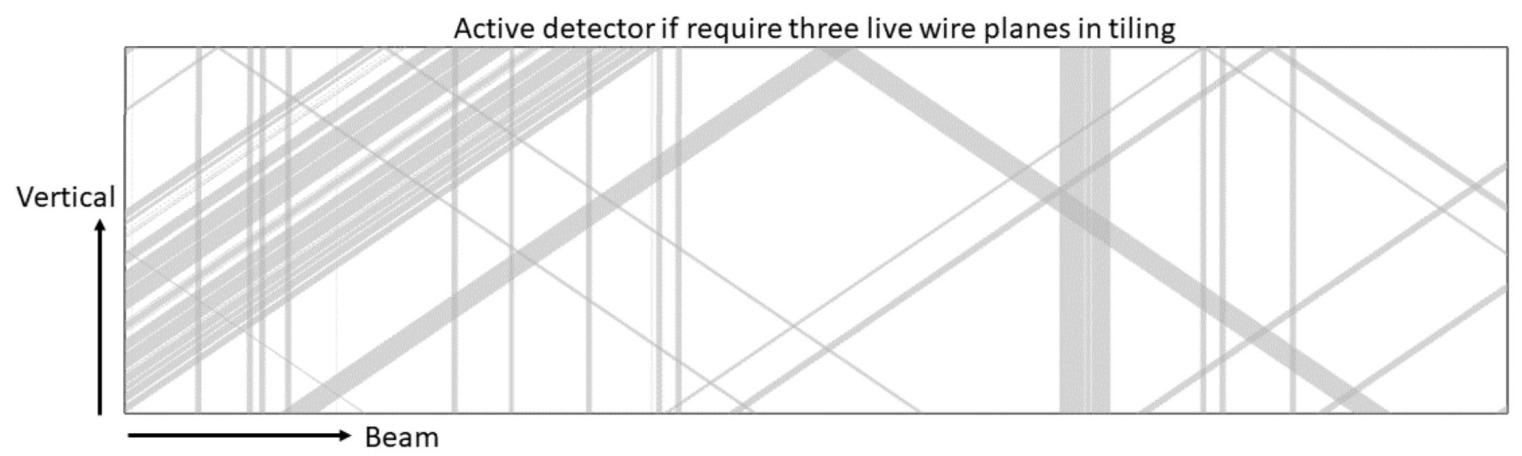

Figure A.1: The nonfunctional regions (gray) in the $\mathrm{Y}-\mathrm{Z}$ plane (anode plane) requiring three active (top) or two active (bottom) wire planes.

LArTPC detectors involves the extraction of charge information from the waveforms on the anode plane wires, as descrivbed in Section 4.2.2. However, the total charge extracted in this way normally does not equal the total charge produced from ionisation for a number of reasons [91].

- Distortions in detector response due to nonfunctional TPC channels.

- Space charge effects (see Appendix B).

- Electron attachment to impurities, diffusion and recombination.

To trace back the exact amount of charge released from the original interaction, we correct for each of these effects starting from the ionisation charge, as reconstructed from the signal collected on the TPC wires.

The first step in the calibration aims to correct the position- and time-dependence of the detector response to ionisation charge using data from ACPT cosmic ray muons. This is done in $5 \mathrm{~cm} \times 5 \mathrm{~cm} \times 10 \mathrm{~cm}$ voxels. Two examples are shown in Figure A.2. The charge calibration correction factors demonstrate the shadowing effects of large continuous regions of malfunction wires on the other planes. See [91] for a detailed treatment of the calibration procedure. 

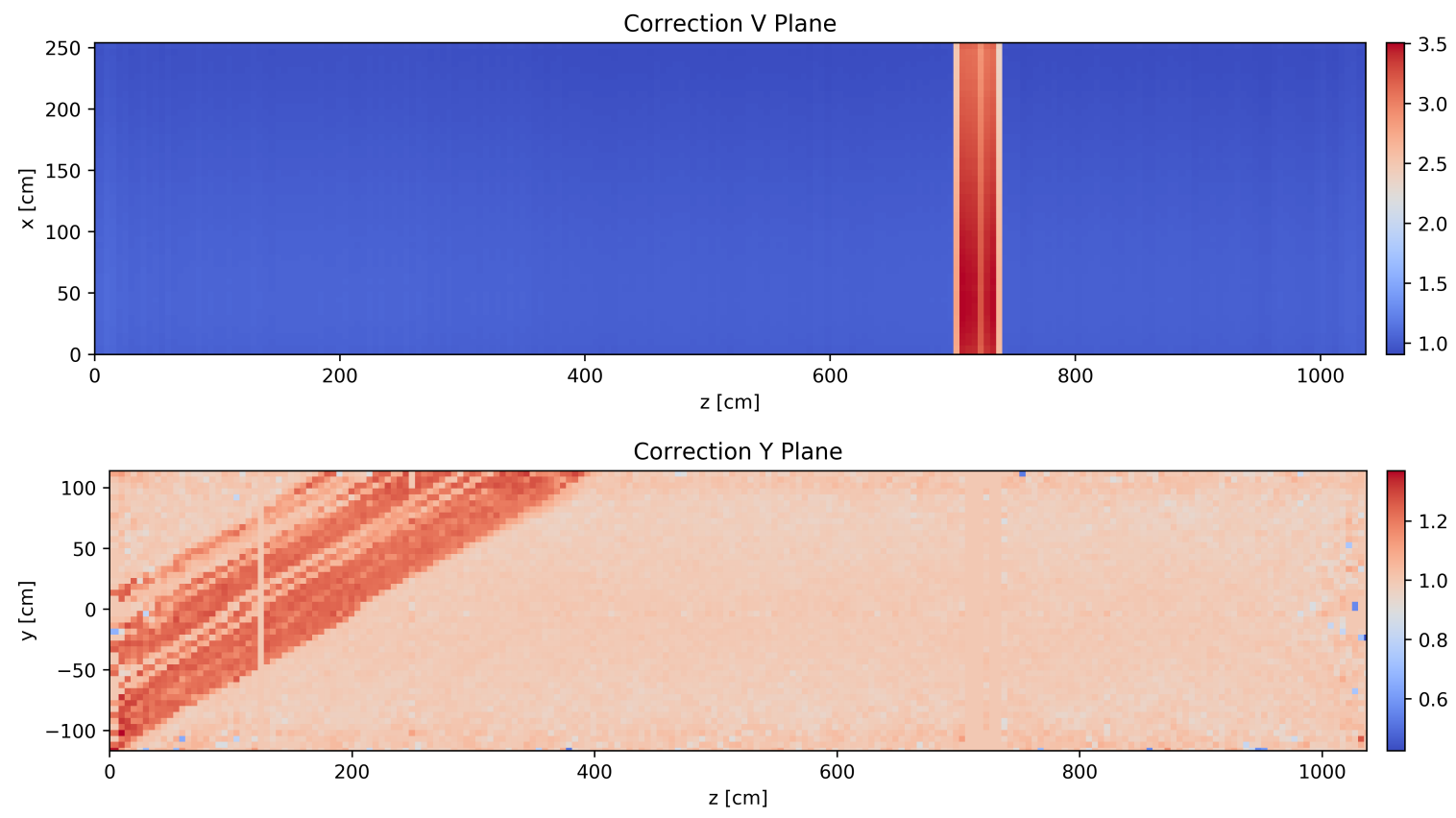

Figure A.2: Position dependent charge calibration factors in simulation for the $Y Z$ projection. Given for the second induction (top) and collection (bottom) wire planes. 


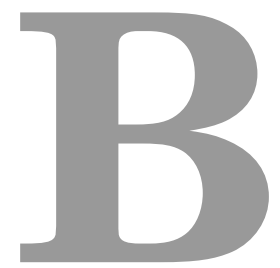

\section{The Space-Charge Effect}

Space charge effect is the build-up of slow-moving positive ions in the TPC. This causes a distortion of the electric field within the detector and mimics a displacement in the reconstructed position of signal ionisation electrons. Additionally, the field distortion causes variations in the amount of charge quenching experienced by ionisation throughout the volume of the TPC [104]. Imagine an interaction at the top (high y) of the active TPC volume. The ionisation electrons will see a build-up of positive charged ions at lower $y$, resulting in an attractive force towards lower $y$. While the electrons are drifting under the influence of the homogeneous electric field towards the anode $(x \approx 0)$, they will be going slightly downwards. Therefore, the charge deposit will be reconstructed at a $y$-position slightly lower than its actual position. Reversing this reasoning explains why a charge deposit at the bottom of the TPC will appear slightly higher after reconstruction. In conclusion, space 
charge effects will appear to compactify the $Y Z$ projection, perpendicular on the drift direction of the active TPC. The effect is stronger at the edges, of the order of $10 \mathrm{~cm}$, and is largely cancelled out in the center.

Figure B.1 shows this effect in the top and bottom of the detector for CORSIKA generated particles and data. The majority of tracks, correspond to downwards through-going muons that are entering from the top $(y=117 \mathrm{~cm})$ and exiting at the bottom $(y=-115 \mathrm{~cm})$. Due to space charge, we see that the region of TPC entrance is smeared out over $\approx 15 \mathrm{~cm}$. A similar effect can be seen at the bottom in the lower two panels of Figure B.1.

It can be seen that the theory-based space charge simulation is not perfect and slightly overestimated, especially at the top of the TPC. The data-driven space charge does a better job in reproducing the asymmetries observed in data but under predicts the strength at the TPC boundaries. 

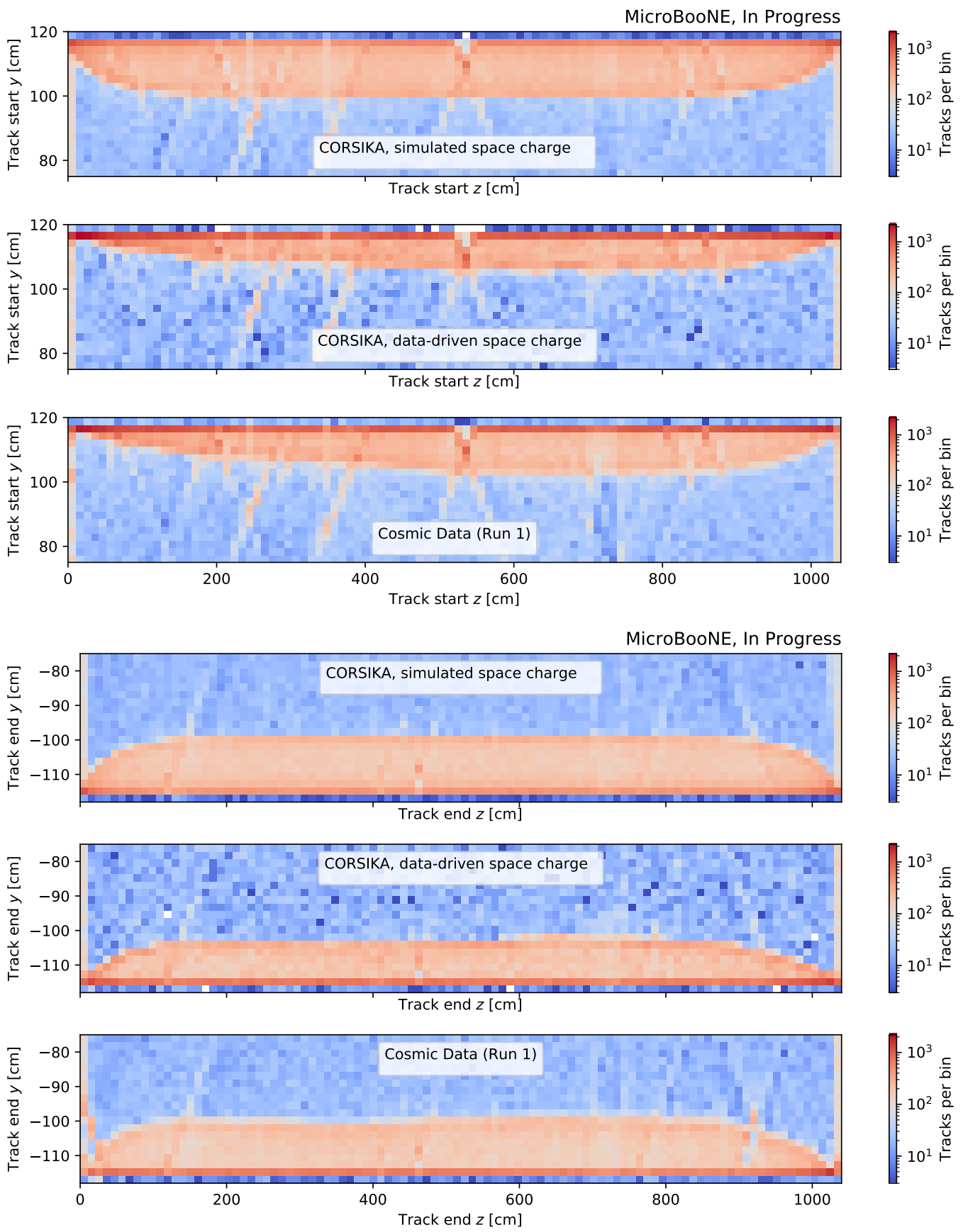

Figure B.1: (Upper three panels) $Y Z$ projection of the reconstructed track start points in the top part of the active TPC for Monte Carlo (first two panels) and data (third panel). (Lower three panels) $Y Z$ projection of the reconstructed track end points in the bottom part of the active TPC for Monte Carlo (panel four and five) and data (sixth panel). A minimum reconstructed track length of $5 \mathrm{~cm}$ is imposed and the $\mathrm{MC}$ is scaled to the data (22k events). 
Appendix B. The Space-Charge Effect 


\section{Data-sets \& Energy Threshold Determination in the Cosmic-ray Measurement}

This appendix serves as a clarification to Chapter 5. Appendix C.1 documents the samples that are used for the cosmic ray measurements. The normalisation between the simulation and data is different compared to the normalisation employed when neutrinos are studied and is explained in Appendix C.2. The last section of this appendix describes the effective energy ranges that are covered by the results shown in Chapter 5.

\section{C.1 Samples}

All Monte Carlo samples are simulated using CORSIKA. The samples have an unbiased trigger and contain only cosmic activity. Different samples are used to estimate systematic 
uncertainties:

- Default LArTF geometry, theory-based space charge, proton-like CORSIKA model (30k events)

- Default LArTF geometry, data-driven space charge, proton-like CORSIKA model (8k events)

- Default LArTF geometry, no simulated space charge, proton-like CORSIKA model (6k events)

- Default LArTF geometry, theory-based space charge, CMC CORSIKA model (8k events)

- Reduced LArTF geometry, data-driven space charge, proton-like CORSIKA model (10k events)

- Increased LArTF geometry, data-driven space charge, proton-like CORSIKA model (10k events)

For the PMT and TPC based rate measurement, a Run 1 sample is used, collected between February and May 2016. This avoids dealing with the effects from a broken PMT in run 3. This sample is not triggered and is guaranteed to be free from any beam neutrino induced activity.

In Section 5.4, a sample of Run 3 data is used to ensure the availability of the CRT information, this sample contains $49 \mathrm{k}$ events collected between the 27th June and the 10th of July in 2018:

\section{C.2 Normalisation}

The cosmic contribution inside the data samples used is unbiased, therefore normalisation is performed based on the total time, summed over the events. In most cases this means that the normalisation factor between data and Monte Carlo corresponds to the number of events in the respective samples. For the optical reconstruction, the reconstruction time 
window is slightly different in data and $\mathrm{MC}$, this is taken into account as was described in Section 5.3. 

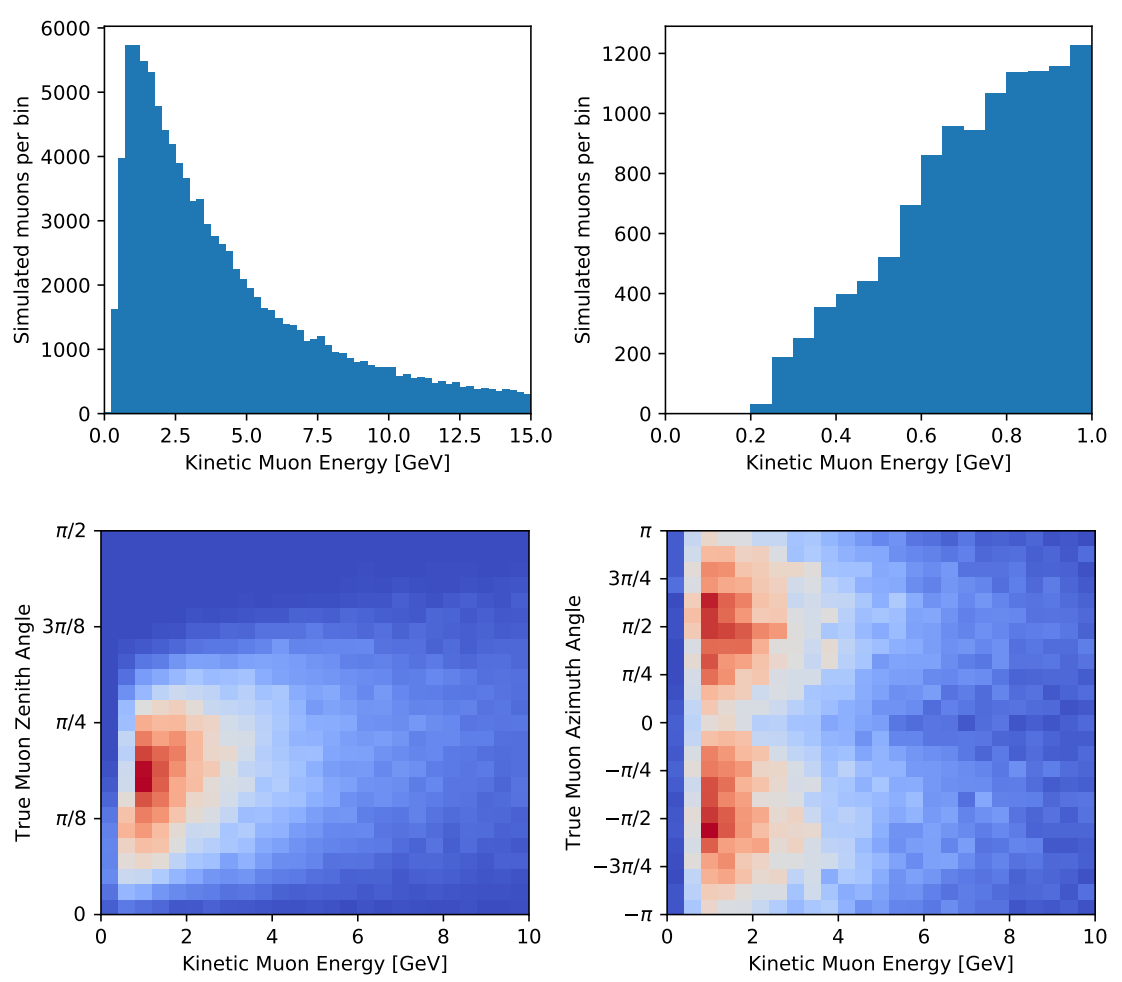

Figure C.1: Energy dependence of cosmic muon track selection. The upper panels show the true energy of simulated muons that are selected ( $25 \mathrm{~cm}$ reconstructed length inside the TPC). The upper right panel is a zoomed in version, it can be seen that there is a minimum kinetic muon energy of $200 \mathrm{MeV}$. Note that the cut-off is not sharp and levels out at $\approx 1 \mathrm{GeV}$. The bottom panels show the relation between the angles of the simulated muon and the energy. Muons coming in at high zenith angle have to traverse dirt around LArTF and therefore have a higher energy threshold.

\section{C.3 Energy threshold and dependence}

The rate measurement presented here is integrated over all angles and energies. As mentioned before, the angular coverage is approximately 0 to 75 degrees in the zenith angle and the full 360 degrees in the azimuthal angle. The limited coverage for horizontal tracks originates from the finite extend of the plane at $y=18 \mathrm{~m}$ where the particles are created. Concerning the energy, the lowest energy per galactic proton is set at $1 \mathrm{GeV}$. Below $1 \mathrm{GeV}$ the resulting shower particles have a low probability of reaching the surface. In $1 \times 10^{6}$ showers, no proton showers that had particles reaching the surface with a primary kinetic energy below $1.4 \mathrm{GeV}$ were found. Nevertheless, due to the TPC being shielded by 

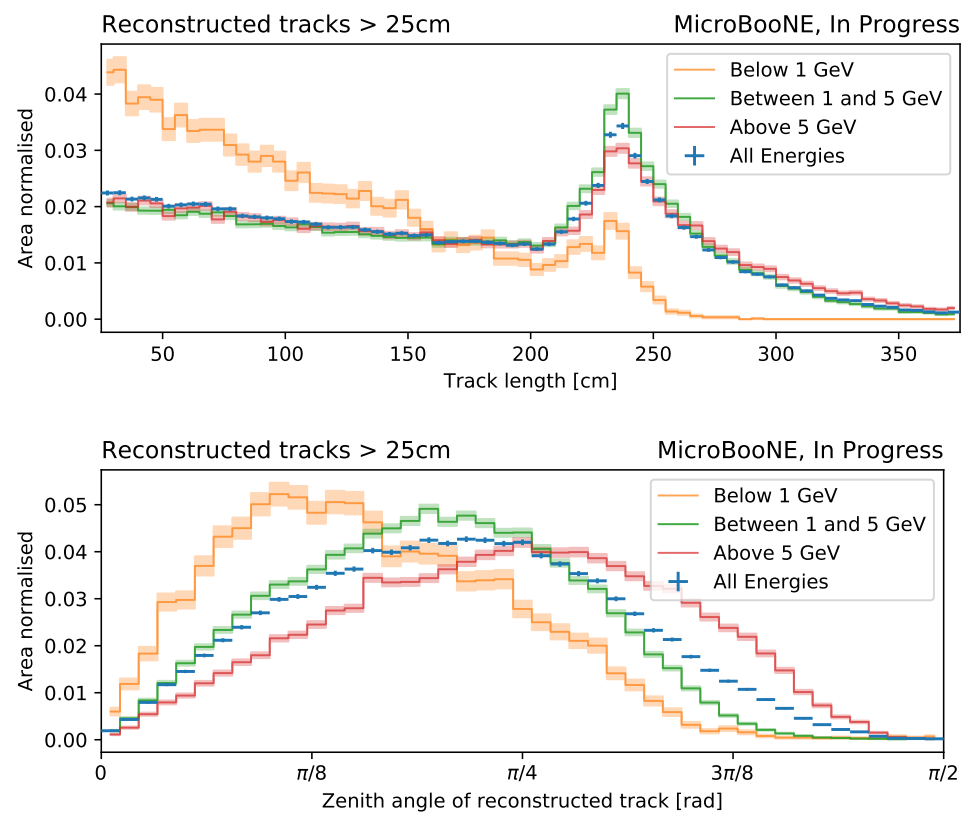

Figure C.2: The distributions of the length inside the TPC (top) and the zenith angle (bottom) of the selected muons depend on the simulated muon energy. Higher energies are required for more horizontal tracks due to the dirt around the LArTF building. To travel longer distances in the TPC, tracks need to be more horizontal due to the TPC geometry. Furthermore, muons are minimising ionising particles and therefore need at least $210 \mathrm{MeV}$ for every meter travelled inside the LAr-filled cryostat.

parts of the cryostat and the environment, the selected muons do have an energy threshold/dependence. This is studied in Figures C.1 and C.2 for the TPC based measurement. It can be seen that the lower threshold for a simulated muon to create a $25 \mathrm{~cm}$ reconstructed track is $\approx 200 \mathrm{MeV}$ kinetic energy.

Figures C.3 and C.4 document the inefficiency and energy threshold effects in flash creation. Although the main threshold effects are coming from geometrical inefficiencies, it can be seen that the minimal muon energy is $\approx 300 \mathrm{MeV}$, corresponding to $\approx 200 \mathrm{MeV}$ kinetic energy.

it can be concluded that the results in this note are valid in a range:

- Zenith angle from 0 to 75 degrees.

- Azimuth angle from -180 to 180 degrees.

- Muon kinetic energy above $\approx 200 \mathrm{MeV}$. 

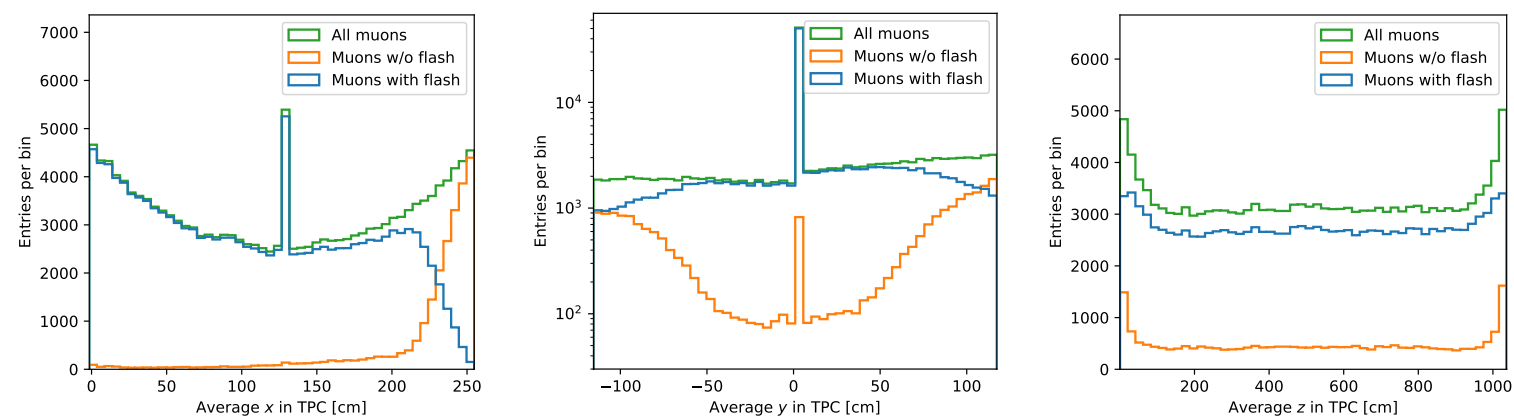

Figure C.3: Threshold effect of flash creation due to the geometrical position of the traversing muon track in the TPC. The location of the mean between the track start and end point are used, leading to central peaks for horizontally and vertically crossing tracks in the $x$ and $y$ direction.
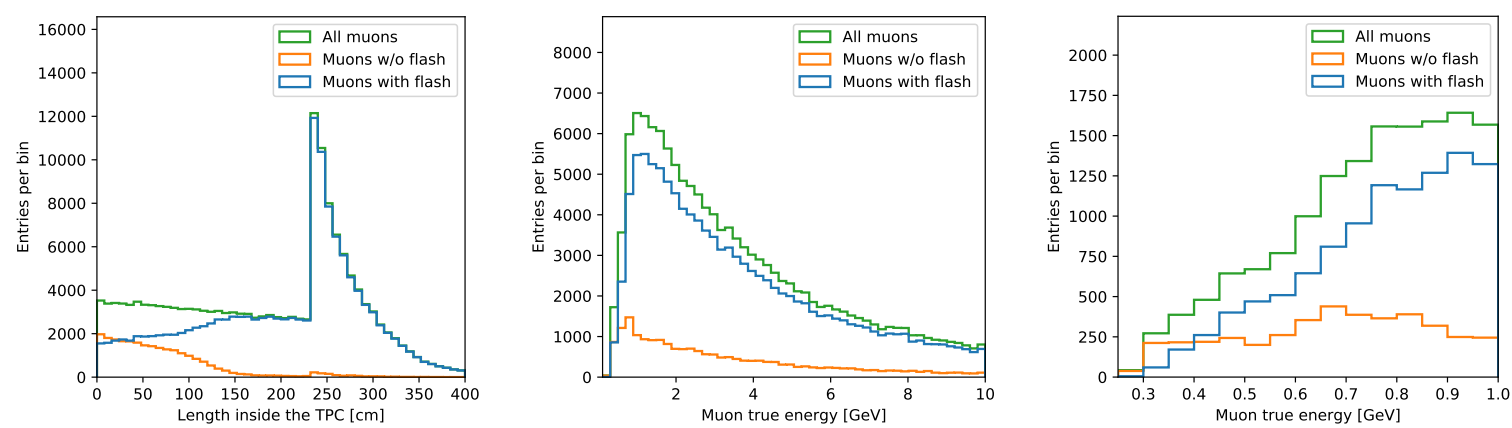

Figure C.4: Threshold effect of flash creation due to the length of the track inside the TPC and the initial muon energy. The panel on the right is a zoomed-in version of the central panel. 


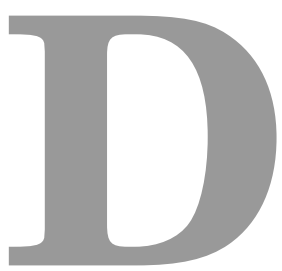

\section{Flux \& Cross-Section Variations}

At the time of writing, the MicroBooNE collaboration has no publicly available documentation supporting the configuration of the flux and cross-section simulation. The flux simulation itself is described in [44] and the neutrino interaction generator, GENIE, is documented in [42]. This appendix lists all the parameters that are varied to estimate the systematic uncertainties on the electron and muon kinematic measurements in Chapter 9.

Variations in the BNB flux simulation The various sources of flux uncertainties are taken as independent and are varied individually around the central value. All sources included in the variation performed in this thesis are listed in Table D.1 along with the number of universes considered for each. 
Table D.1: List of flux variations used in Chapter 9 to estimate the systematic uncertainties on the lepton kinematics.

\begin{tabular}{|c|c|c|}
\hline Parameter Name & Description & Universes \\
\hline expskin_FluxUnisim & $\begin{array}{l}\text { skin-depth electric currents } \\
\text { penetrate conductor }\end{array}$ & 50 \\
\hline horncurrent_FluxUnisim & $\begin{array}{l}\text { horn current in magnetic fo- } \\
\text { cusing horns }\end{array}$ & 50 \\
\hline kminus_PrimaryHadronNormalization & $\begin{array}{l}\text { Primary hadron normaliza- } \\
\text { tion }\end{array}$ & 50 \\
\hline kplus_PrimaryHadronFeynmanScaling & $\begin{array}{l}\text { Primary Hadron Feynman } \\
\text { Scaling }\end{array}$ & 50 \\
\hline kzero_PrimaryHadronSanfordWang & $\begin{array}{l}\text { Primary Hadron Sanford } \\
\text { Wang }\end{array}$ & 50 \\
\hline nucleoninexsec_FluxUnisim & $\begin{array}{l}\text { nucleon total inelastic cross } \\
\text { section on Be }\end{array}$ & 50 \\
\hline nucleonqexsec_FluxUnisim & $\begin{array}{l}\text { nucleon total quasi-elastic } \\
\text { cross section on Be }\end{array}$ & 50 \\
\hline nucleontotxsec_FluxUnisim & $\begin{array}{l}\text { nucleon total cross section on } \\
\text { Be }\end{array}$ & 50 \\
\hline piminus_PrimaryHadronSWCentralSplineVariation & $\begin{array}{l}\text { Primary Hadron Sanford } \\
\text { Wang Central Spline Varia- } \\
\text { tion }\end{array}$ & 50 \\
\hline pioninexsec_FluxUnisim & $\begin{array}{l}\text { pion total inelastic cross sec- } \\
\text { tion on Be }\end{array}$ & 50 \\
\hline pionqexsec_FluxUnisim & $\begin{array}{l}\text { pion total quasi-elastic cross } \\
\text { section on Be }\end{array}$ & 50 \\
\hline piontotxsec_FluxUnisim & pion total cross section on Be & 50 \\
\hline piplus_PrimaryHadronSWCentralSplineVariation & $\begin{array}{l}\text { Primary Hadron Sanford } \\
\text { Wang Central Spline Varia- } \\
\text { tion }\end{array}$ & 50 \\
\hline
\end{tabular}

Variations in the neutrino interaction modelling A total of 55 sources of uncertainties are considered. The majority of them, 49 , are varied in a multisim way around the updated GENIE central value tune. Their variations are simultaneous to take into account their correlations. This simultaneous variation is given the name All_Genie. They are listed in Table D.3. The variations of the remaining six sources cover the full range of the parameter values: they are varied from 0 to 1 . Table D.2 lists the uncertainties that are used in the analysis and the number of universes investigated. 
Table D.2: List of GENIE systematic variations used used in Chapter 9 to estimate the systematic uncertainties on the lepton kinematics.

\begin{tabular}{llc}
\hline \multicolumn{1}{c}{ GENIE knobs } & \multicolumn{1}{c}{ Description } & Universes \\
\hline All_Genie & GENIE knobs listed in Table D.3 & 100 \\
RPA_CCQE_Genie & Strength of the RPA correction & 2 \\
XSecShape_CCMEC_Genie & Changes shape of CCMEC differential cross section & 2 \\
AxFFCCQEShape_Genie & CCQE Axial form factor model & 2 \\
VecFFCCQEshape_Genie & CCQE vector form factor model & 2 \\
DecayAngMEC_Genie & Changes angular distribution of nucleon cluster & 2 \\
Theta_Delta2Npi_Genie & Interpolates angular distribution for $\Delta \rightarrow N+\pi$ be- & 2 \\
& tween Rein-Sehgal model $(0)$ and isotropic $(1)$ & \\
\hline
\end{tabular}


Table D.3: Uncertainties included in All_Genie, see Table D.2

\begin{tabular}{|c|c|}
\hline GENIE uncertainties & Description \\
\hline MaCCQE & CCQE axial mass \\
\hline NormCCMEC & Energy-independent normalization for CCMEC \\
\hline MaNCEL & Axial mass for NCEL \\
\hline EtaNCEL & $\begin{array}{l}\text { Empirical parameter used to account for sea quark contribution to NCEL form } \\
\text { factor }\end{array}$ \\
\hline NormNCMEC & Energy-independent normalization for NCMEC \\
\hline FracPN_CCMEC & Varies fraction of initial nucleon pairs that are pn \\
\hline FracDelta_CCMEC & Varies relative contribution of $\Delta$ diagrams to total MEC cross section \\
\hline NormCCRES & Energy-independent normalization for CCRES \\
\hline MaCCRESshape & Shape-only CCRES axial mass \\
\hline MvCCRESshape & Shape-only CCRES vector mass \\
\hline NormNCRES & Energy-independent normalization for NCRES \\
\hline MaNCRESshape & Shape-only NCRES axial mass \\
\hline MvNCRESshape & Shape-only NCRES vector mass \\
\hline MaCOHpi & Axial mass for $\mathrm{COH} \pi$ production \\
\hline R0COHpi & Nuclear radius parameter for $\mathrm{COH} \pi$ production \\
\hline NonRESBGvpCC1pi & Non-resonant background normalization for $v p \mathrm{CC} 1 \pi$ \\
\hline NonRESBGvpCC2pi & Non-resonant background normalization for $v \mathrm{p} C \mathrm{C} 2 \pi$ \\
\hline NonRESBGvpNC1pi & Non-resonant background normalization for $v p \mathrm{NC} 1 \pi$ \\
\hline NonRESBGvpNC2pi & Non-resonant background normalization for $v \mathrm{p} N C 2 \pi$ \\
\hline NonRESBGvnCC1pi & Non-resonant background normalization for $\nu$ n CC $1 \pi$ \\
\hline NonRESBGvnCC2pi & Non-resonant background normalization for $v$ n CC $2 \pi$ \\
\hline NonRESBGvnNC1pi & Non-resonant background normalization for $\nu \mathrm{n} \mathrm{NC} 1 \pi$ \\
\hline NonRESBGvnNC2pi & Non-resonant background normalization for $\nu \mathrm{n} \mathrm{NC} 2 \pi$ \\
\hline NonRESBGvbarpCC1pi & Non-resonant background normalization for $\bar{v} \mathrm{p} C \mathrm{C} 1 \pi$ \\
\hline NonRESBGvbarpCC2pi & Non-resonant background normalization for $\bar{\nu} \mathrm{p} C \mathrm{C} 2 \pi$ \\
\hline NonRESBGvbarpNC1pi & Non-resonant background normalization for $\bar{v} \mathrm{p} N C 1 \pi$ \\
\hline NonRESBGvbarpNC2pi & Non-resonant background normalization for $\bar{v} \mathrm{p} N C 2 \pi$ \\
\hline NonRESBGvbarnCC1pi & Non-resonant background normalization for $\bar{\nu} \mathrm{n} C \mathrm{C} 1 \pi$ \\
\hline NonRESBGvbarnCC2pi & Non-resonant background normalization for $\bar{\nu} \mathrm{n} C \mathrm{C} 2 \pi$ \\
\hline NonRESBGvbarnNC1pi & Non-resonant background normalization for $\bar{\nu} \mathrm{n} N C 1 \pi$ \\
\hline NonRESBGvbarnNC2pi & Non-resonant background normalization for $\bar{\nu} \mathrm{n} \mathrm{NC} 2 \pi$ \\
\hline AhtBY & A_HT higher-twist parameter in the Bodek-Yang model scaling variable xi_w \\
\hline BhtBY & BHT higher-twist parameter in the Bodek-Yang model scaling variable xi_w \\
\hline CV1uBY & CV1u valence GRV98 PDF correction parameter in the Bodek-Yang model \\
\hline CV2uBY & CV2u valence GRV98 PDF correction parameter in the Bodek-Yang model \\
\hline AGKYxF1pi & Hadronization parameter, applicable to true DIS interactions only \\
\hline AGKYpT1pi & Hadronization parameter, applicable to true DIS interactions only \\
\hline MFP_pi & $\pi$ mean free path \\
\hline MFP_N & Nucleon mean free path \\
\hline FrCEx_pi & Fractional cross section for $\pi$ charge exchange \\
\hline FrInel_pi & Fractional cross section for $\pi$ inelastic scattering \\
\hline FrAbs_pi & Fractional cross section for $\pi$ absorption \\
\hline FrCEx_N & Fractional cross section for nucleon charge exchange \\
\hline FrInel_N & Fractional cross section for nucleon inelastic scattering \\
\hline FrAbs_N & Fractional cross section for nucleon absorption \\
\hline RDecBR1gamma & Normalization for $\Delta \rightarrow \gamma$ decays \\
\hline RDecBR1eta & Normalization for $\Delta \rightarrow \eta$ decays \\
\hline FrPiProd_pi & Fractional cross section for $\pi^{-}$induced $\pi$ production \\
\hline FrPiProd_N & Fractional cross section for nucleon-induced $\pi$ production \\
\hline
\end{tabular}




\section{Acronyms}

ACPT Anode or Cathode Piercing Tracks. 110, 116-118, 232, 254

ADC Analogue-to-Digital Converter. 62, 63, 65, 115, 124

ASIC Application-Specific Integrated Circuit. 44, 66, 231

AUC Area Under the ROC Curve. 183

BDT Booster Decision Tree. 181-184, 186-192, 194, 256, 259

Beam Off Data acquired with beam off but with the same run configuration and trigger as Beam On. 60, 61, 106, 107, 110, 111, 113, 115, 121, 128, 129, 133, 134, 137, 138, 143, 147, $151,170,193,203,253,255$

Beam On Data acquired with beam on and triggered by the light system. 60, 107, 108, 110, $111,129,133,134,137,193,249,253,255$

BNB Booster Neutrino Beam. 19, 36-39, 42, 50, 53, 57, 58, 60-62, 64, 107, 110, 111, 113, 122, 137, 142-145, 155, 161, 164-166, 182, 201-203, 205, 206, 218, 222, 223, 228, 229, 245, 253, $254,256,259$

CC Charged Current. 13, 22, 30-33, 165, 167, 206, 212, 213, 218

CMC Constant Mass Composition. 76, 77, 101, 102

CORSIKA COsmic Ray SImulations for KAscade. 51, 70, 75-78, 82, 83, 87-89, 91-94, 97, 98, 101-103, 236

CRT Cosmic Ray Tagger. 43, 51, 52, 73, 98-101, 105, 137, 138, 141, 143, 144, 155, 170, 193, 194, 240, 253

CRY Cosmic-RaY shower library. 75-77

DAQ Data Acquisition. 41, 62, 132, 133, 135, 136

DIS Deep Inelastic Scattering. 32, 33, 153, 164

DUNE Deep Underground Neutrino Experiment. 14, 36, 54

event A MicroBooNE data-event is defined as a stored set of data-products corresponding to a trigger $(t=0)$. An event compromises TPC readout from -0.4 to $2.8 \mathrm{~ms}$ and PMT information covering the TPC readout window. For more recent data, an event also includes information recorded by the CRT system. 45, 74, 82, 107, 112 
Fermilab Fermi National Accelerator Laboratory. 14, 19, 35-37, 39, 55, 56, 74, 75, 77, 101, 253

flash Collection of coincidental optical hits over the 32 PMT's in MicroBooNE. The light is integrated over 8 microseconds and the intensity of all hits is summed. A reconstructed flash object aims to represent the light coming from a single interaction in the TPC. $64,92,110,111,137,208$

FSI Final State Interactions. 29, 31-33, 143, 206, 253

FV Fiducial Volume. 106, 107, 114, 118, 119, 122, 133, 145, 147, 171, 254

GALLEX Gallium Experiment. 24, 26

GEANT GEometry ANd Tracking: A platform for the simulation of the passage of particles through matter. 51, 70, 71, 77, 83, 101, 116, 203

GENIE The neutrino event generator. 70, 202, 205-207, 213-217, 245-248, 259

ICARUS Son of Daedalus who dared to fly too near the sun on wings of feathers and wax. 57

KARMEN KArlsruhe Rutherford Medium Energy Neutrino experiment. 18, 19

KATRIN KArlsruhe TRItium Neutrino experiment. 7

KS-test Kolmogorov-Smirnov test. 158, 183

LAr Liquid Argon. 24, 35, 41, 43, 47, 49, 50, 53-56, 118, 119, 137, 141, 142, 161, 165, 173, 189, $194,227,229,232,253,256$

LArTF Liquid Argon Test Facility. 41, 74, 75, 78, 90, 91, 95, 96, 101, 102, 253, 254

LEE Low Energy Excess. 23, 35, 53, 130, 134, 143, 164, 166-169, 171, 183, 212, 223, 225, 226, 255,256

LLR Log-Likelihood Ratio. 222, 225, 226

LSND Liquid Scintillator Neutrino Detector. 18-20, 22, 25, 26, 57

MC Monte Carlo. 73, 75, 82, 83, 85-91, 95, 96, 99, 100, 102, 107, 108, 113-116, 118, 121, 125, $128,130,132,145,151,157,168,169,176,181,183,188,190,207,213,215,237,241,254$, 256,259

MCS Multiple Coulomb Scattering. 118-120, 157-160, 254, 255

MEC Meson Exchange Current. 30, 32, 161, 164, 206

MiniBooNE Mini Booster Neutrino Experiment. 1, 2, 18-23, 25, 26, 32, 35, 40, 53, 164, $166-169,183,205,212,223,225,229,253,255$

MIP Minimal Ionising Particle. 45, 79, 173 
MSW Mikheyev-Smirnov-Wolfenstein. 11, 13, 14

NC Neutral Current. 23, 30, 31, 145-147, 165-167, 175, 188, 212, 255

NeutrinoID Set of tools to identify neutrino interactions and reject cosmic activity by combining information from the different MicroBooNE sub-detectors. 105, 106, 109, 111, 123, 127, 129-136, 142, 144, 146, 147, 154, 166, 171, 193-195, 197, 198, 208, 254-256, 259

NuMI Neutrinos from the Main Injector. 36, 37, 39, 40, 51, 60, 228

Overlay Monte Carlo sample generated with GENIE simulated $v$ and cosmic unbiased data as background. 107, 108, 180, 182, 195, 203

PCA Principal Components Analysis. 123

PDG Particle Data Group. 119

PE Photo-Electrons. 62-64, 92, 93, 96, 110-112, 115, 124, 125, 130, 209

PID Particle Identification. 148, 151, 152, 159, 161, 187-189, 191, 210, 211, 255, 256

PMT Photo-Multiplier Tube. 20, 21, 41-43, 49-51, 59, 61-64, 73, 92, 94, 96, 97, 101-103, 112, $116,124-126,130,209,240,253$

PNMS Pontecorvo-Maki-Nakagawa-Sakata. 13, 15

POT Protons on Target. 22, 38, 57, 60, 107, 132-136, 141, 155, 193, 199, 213, 214, 216, 218, $222,229,256$

QE Quasi-Elastic scattering. 30, 31, 153, 161, 164, 167, 206, 213, 218

RAA Reactor Antineutrino Anomaly. 24, 26

RES Resonant production. 33, 161, 164

ROC Receiver Operating Characteristic. 182, 183, 249

ROI Region of Interest. 66

SAGE Soviet-American Gallium Experiment. 24, 26

SBN Short-Baseline Neutrino Program at Fermilab. 56, 57, 229, 253

SBND Short-Baseline Near Detector. 56

slice A collection of distinct reconstructed Pandora particles which are believed to belong to the same interaction in the TPC. $67,109-112,120,123,188,192,196,197$

SoLid Short baseline Oscillation search with Lithium-6 Detector. 25

SVM Support Vector Machine. 68, 69, 110, 122, 123 
TPB Tetra-Phenyl Butadiene. 50, 51, 124

TPC Time Projection Chamber. 24, 35, 41-46, 49-51, 53-56, 59-67, 73-75, 77-83, 86, 87, 90, 92-94, 96-98, 101-103, 106-110, 116, 118, 119, 124, 137, 138, 141, 142, 144-146, 161, 165, $177,189,194,201,208,210,211,227,229,231,232,235-237,240,253,254,256$

VUV Vacuum Ultra-Violet (10 to 200 nanometer). 48, 51 


\section{List of Figures}

2.1 The Standard Model of elementary particles . . . . . . . . . . . . . . . 6

2.2 Neutrino helicity in the Standard Model . . . . . . . . . . . . . . . . . . 7

2.3 Electron mass term Feynman representation . . . . . . . . . . . . . . . 8

2.4 LSND neutrino production . . . . . . . . . . . . . . . . . . . 19

2.5 Signature of a neutrino event in LSND . . . . . . . . . . . . . . . . 20

2.6 Particle Identification in MiniBooNE . . . . . . . . . . . . . . . . . . . . . 21

2.7 MiniBooNE's low energy excess . . . . . . . . . . . . . . . . . . 22

2.8 Short-baseline reactor antineutrino anomaly $\ldots \ldots \ldots \ldots$

2.9 Final State Interactions (FSI) . . . . . . . . . . . . . . . . . . . . . . . . . . . . . 29

2.10 Charged current quasielastic scattering on argon . . . . . . . . . . . . . . . 31

2.11 Charged current MEC scattering on argon . . . . . . . . . . . . . . . . . . . 32

2.12 Charged current RES scattering on argon . . . . . . . . . . . . . . 33

2.13 Charged current DIS scattering on argon . . . . . . . . . . . . 33

3.1 The Fermilab accelerator complex . . . . . . . . . . . . . . . . . . . 37

3.2 The Booster Neutrino Beam at Fermilab . . . . . . . . . . . . . . . . 37

3.3 BNB energy spectrum in neutrino mode . . . . . . . . . . . . . . . . . . 39

3.4 BNB breakdown of hadronic contributions . . . . . . . . . . . . . . . 39

3.5 Picture of MicroBooNE during installation and model of the MicroBooNE detector in LArTF . . . . . . . . . . . . . . . . . . . . 41

3.6 Drawing of the MicroBooNE cryostat and coordinate system . . . . . . . . . 42

3.7 LArTPC technology . . . . . . . . . . . . . . . . . . . . . . . 43

3.8 Picture of the MicroBooNE TPC \& signals on the wire planes . . . . . . . . . . 45

3.9 MicroBooNE example data neutrino event on the collection plane . . . . . . . 46

3.10 Scintillation light production in Liquid Argon . . . . . . . . . . . . . . . . . . 47

3.11 Excimer state energies as a function of nuclear separation . . . . . . . . . . . 48

3.12 Decrease of light yield in MicroBooNE, interpreted as a decrease in the absorption length by impurities . . . . . . . . . . . . . . . . . . . 49

3.13 Schematic and picture of the MicroBooNE PMT system . . . . . . . . . . . . . 50

3.14 Emission and absorption spectra in Liquid Argon . . . . . . . . . . . . . . 51

3.15 The design of the Cosmic Ray Tagger system . . . . . . . . . . . . . . . . . 52

3.16 Breakdown of neutrino interactions in MicroBooNE by different nuclear interaction types . . . . . . . . . . . . . . . . 54

3.17 Map of the Short-Baseline Neutrino Program at Fermilab . . . . . . . . . . . 56

3.18 Sensitivity of SBN to the sterile neutrino hypothesis . . . . . . . . . . . 57

3.19 Sterile neutrino signal example in SBN . . . . . . . . . . . . . . . 58

4.1 Beam On and Beam Off type of events. . . . . . . . . . . . . . . . . . 61 
4.2 Example of a flash consisting of multiple PMT hits combined . . . . . . . . . 64

4.3 Signal processing and $2 D$ deconvolution $\ldots \ldots \ldots \ldots \ldots$

4.4 The Pandora output data products . . . . . . . . . . . . . . . 67

4.5 Reconstructed electron neutrino in Monte Carlo . . . . . . . . . . . . . . 68

5.1 Simulation of cosmic particles in MicroBooNE and their end points . . . . . 75

5.2 Comparisons of the energy distributions for different cosmic generators . . . 77

5.3 Concrete elements in the LArTF simulated geometry . . . . . . . . . . . 78

5.4 Simulated muon kinetic energy and stopping muon fraction . . . . . . . . . 80

5.5 Coordinate frame and angle definitions for muon studies in the TPC . . . . . 83

5.6 Reconstruction resolution for the track length, zenith angle and azimuthal angle .......................... 84

5.7 Muon reconstruction resolution example . . . . . . . . . . . . . . 85

5.8 Fractional resolution and confidence intervals for reconstructed muons . . . . 86

5.9 Reconstructed cosmic muon track length . . . . . . . . . . . . . . 87

5.10 Reconstructed cosmic muon azimuth angle . . . . . . . . . . . . . 88

5.11 Reconstructed cosmic muon zenith angle . . . . . . . . . . . . . . . . 89

5.12 Effect of systematic uncertainties on the TPC-based cosmic muon measurement 91

5.13 The connection between CORSIKA simulated particles and reconstructed flashes . . . . . . . . . . . . . . . . . . . 93

5.14 Flash reconstruction efficiency for comic muon as a function of $x \ldots \ldots 4$

5.15 Centre of the reconstructed flash for cosmogenic muons . . . . . . . . . . . 97

5.16 Location and layout of the bottom cosmic ray tagging panel . . . . . . . . . 98

5.17 CRT bottom panel simulated and data hit densities . . . . . . . . . . . . . 99

5.18 Exponential fit to time intervals of sequential CRT hits in simulation and data 100

5.19 Comparison of the muon flux measurement in this work with previous work 103

5.20 The magnitude of the seasonal flux variations at different detector depths . . 103

6.1 Fiducial Volume (FV) used in this thesis. The yellow cylinder represents the cryostat, the orange structure is the TPC and the green area is the FV. Both the $Y Z$ (left) and $Y Z$ (right) projections are shown. . . . . . . . . . . . 107

6.2 Schematic of a typical event and the succession of steps to reject cosmic activity109

6.3 BNB spill flash times . . . . . . . . . . . . . . . . . . . . 111

6.4 Schematic defining the slice purity and completeness . . . . . . . . . . . 112

6.5 Requirement of the position of the beam flash and the centre of the charge in $z 113$

6.6 Requirement of the position of the beam flash and the centre of the charge in $y 114$

6.7 $2 D$ scatter and density plots connecting the $x$-position and the collection charge of the slice with the flash intensity. . . . . . . . . . . . . . 115

6.8 Rectangular cut combining the flash intensity with the collected charge and $x$-position for the slice. . . . . . . . . . . . . . . 116

6.9 Rectangular cut aimed at removing Anode or Cathode Piercing Tracks events 117

6.10 Rectangular cut aimed at removing Anode or Cathode Piercing Tracks . . . . 118

6.11 Illustrative description of Multiple Coulomb Scattering . . . . . . . . . . . . 119

6.12 Multiple Coulomb Scattering likelihood to identify stopping muon background. . . . . . . . . . . . . . . . . . . . . 120

6.13 Number of neutrino candidates before and after the slice rejection steps . . 121 6.14 Pandora topological score of the slices passing the NeutrinoID . . . . . . . . 123 
6.15 Illustration of the steps in the flash-matching process . . . . . . . . . . . 125

6.16 Data/MC comparison of the Flash-match $\chi^{2}$-like metric . . . . . . . . . . . 125

6.17 Example of the flash-matching procedure in Monte Carlo . . . . . . . . . . . 126

6.18 Relation between the topological score, the flash-match $\chi^{2}$ and the neutrino energy . . . . . . . . . . . . . . . . . . 127

6.19 Beam Off rejection using the obvious cosmic flash-matching ratio . . . . . . . 128

6.20 NeutrinoID efficiency as a function of neutrino energy for sub channels of $v_{\mu}$ CC and $v_{e}$ CC events. . . . . . . . . . . . . . . . . 129

6.21 Distributions of the charge centre after the NeutrinoID . . . . . . . . . . . 131

6.22 NeutrinID efficiency as a function of neutrino energy and vertex $x$ location . . 132

6.23 Run numbers in Beam On and Beam Off samples used to validate the NeutrinoID . . . . . . . . . . . . . . . . . . . . 133

6.24 Performance and stability of the NeutrinoID ove different data-sets . . . . . 135

6.25 Ratio of passing rates in verious steps of the NeutrinoID over the data-set . . 136

6.26 The CRT veto: flash-CRT matching and the impact on simulated neutrinos . . 137

6.27 CRT intersections as used by the veto to reject neutrino interactions outside the cryostat . . . . . . . . . . . . . . . . . 138

6.28 Example event rejected by the CRT distance tagger . . . . . . . . . . . . . . . 139

7.1 Flowchart indicating the different steps in the $v_{\mu}$ CC selection . . . . . . . 144

7.2 Fiducial volume applied on the reconstructed vertices . . . . . . . . . . . . 146

7.3 Additional cosmic rejection in the $v_{\mu}$ CC selection . . . . . . . . . . . . . 147

7.4 Muon identification: track score \& vertex distance . . . . . . . . . . . . . . . 148

7.5 The expected $\mathrm{d} E / \mathrm{d} x$ distribution for track-lie particles . . . . . . . . . . . . 150

7.6 Muon identification: track length and PID loglikelihood . . . . . . . . . . . . 151

7.7 Relation between the muon range and its kinetic energy / momentum . . . . 152

7.8 Efficiency of the inclusive $v_{\mu} \mathrm{CC}$ selection for different interaction modes and channels . . . . . . . . . . . . . . . . . . . . 153

7.9 Track multiplicity (left)) and muon candidate angle w.r.t the beam direction (right) for the inclusive $v_{\mu}$ CC selection . . . . . . . . . . . . . . . . . 154

7.10 Efficiency of the contained $v_{\mu}$ CC selection for different interaction modes and channels . . . . . . . . . . . . . . . . . . . . . . 156

7.11 Efficiency of both $v_{\mu}$ CC selections, as a function of the muon energy $\ldots . . .157$

7.12 Comparison of MCS- and range-based momentum estimations of the muon candidate in the contained charged-current muon selection . . . . . . . . 158

7.13 Muon resolution comparison in the contained $v_{\mu}$ CC selection . . . . . . . 159

7.14 Correlation matrix of the variables of interest in the $v_{\mu}$ CC analysis . . . . 160

7.15 Demonstration of exclusive $v_{\mu}$ CC selections for three different final states . . 162

8.1 Breakdown of $v_{e} \mathrm{CC}$ events by interaction modes and final state topologies as a function of neutrino energy . . . . . . . . . . . . . 165

8.2 Flowchart indicating the different steps in the $v_{e}$ CC selection . . . . . . 166

8.3 Example of a NC $\Delta \rightarrow N \gamma$ decay $\ldots \ldots \ldots \ldots$. . . . . . . . . 167

8.4 Unfolding of the MiniBooNE LEE under the electron-like and photon-like hypothesis . . . . . . . . . . . . . . . . . . . 168

8.5 Additional cosmic rejection in the $v_{e}$ CC selection . . . . . . . . . . . . 169

8.6 Electron shower candidate identification in the $v_{e}$ CC selection . . . . . . . 170 
8.7 Electron shower candidate $\theta$ and $\phi$ after the preselection $\ldots \ldots \ldots$. . . . . 172

8.8 Cross section of gammas on argon between $1 \mathrm{MeV}$ and $1 \mathrm{GeV}$. . . . . . . . 174

8.9 Electromagnetic shower formation and $\pi^{0}$ xample in the MicroBooNE LArTPC175

8.10 Subset of variables used for electron identification. . . . . . . . . . . . . . 178

8.11 Simulated examples of muons decaying to Michel electrons, reconstructed as shower objects . . . . . . . . . . . . . . . . . . . 178

8.12 The second shower based $\pi^{0}$ rejection variables. . . . . . . . . . . . . . 180

8.13 Flowchart representing the iterative aspect of the gradient boosting training cycle. Each iteration is called a training epoch. Figure from [102]. . . . . . . 182

8.14 Evaluation of training performance for electron shower identification . . . . . 183

$8.15 e-\gamma$ separation performance obtained by the electron classifier on simulation 184

8.16 Data/MC comparisons for the input variables of the electron shower classifier, as listed in Table 8.2 and its response (bottom right panel). . . . . . . . . 186

8.17 Correlation matrix of the variables of interest in the electron identification step 187

8.18 Evaluation of training performance for the other daughters in the event . . . 190

8.19 Data/MC comparisons for the input variables of the other daughters classifier. The bottom row shows the response of the BDT . . . . . . . . . . . . 191

8.20 Variables used for the final $v_{e}$ CC event selection classifier . . . . . . . . . 192

8.21 Evaluation of training performance for the $v_{e}$ CC event classification . . . . . 193

8.22 Final $v_{e}$ CC event classifier and reconstructed electron energy spectrum . . . . 194

8.23 Efficiency of the different stages in the $v_{e}$ CC selection as a function of the true neutrino energy . . . . . . . . . . . . . . . . . . 195

8.24 Reconstruction quality metrics for $v_{e}$ CC signal events passing the NeutrinoID 198

8.25 Displays of selected $v_{e}$ CC candidate events by the selection described in this chapter. . . . . . . . . . . . . . . . . . . . . . . . . 199

$9.1 v_{\mu}-v_{e}$ BNB flux correlation matrix. Figure from [44]. . . . . . . . . . . . 206

9.2 Muon and electron charge-current interaction rates using different GENIE models . . . . . . . . . . . . . . . . . . . 207

9.3 Effect of light modelling variations . . . . . . . . . . . . . . . . . . . 209

9.4 Impact of TPC waveform variations $(\mathrm{a}, \mathrm{b}, \mathrm{d}, \mathrm{e})$ and the space charge effect variations (c) on the measured electron $\mathrm{d} E / \mathrm{d} x$ after the $v_{e}$ CC preselection. . . . . 210

9.5 Variation in track log likelihood PID, for select TPC response variations. The color scale is logarithmic. . . . . . . . . . . . . . . . . . . . 211

9.6 Lepton kinematics for the $v_{\mu} \mathrm{CC}$ channel. Both resolution and data/MC comparisons are shown . . . . . . . . . . . . . . . . . . . . 215

9.7 Lepton kinematics for the $v_{e} \mathrm{CC}$ channel. Both resolution and data/MC comparisons are shown . . . . . . . . . . . . . . . . 216

9.8 Covariance and derived matrices to perform the data-driven constraint of the systematic uncertainties . . . . . . . . . . . . . . . 217

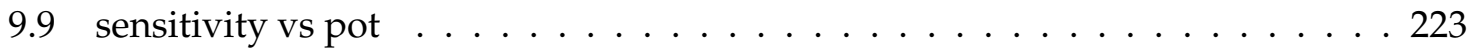

9.10 Effect on the electron neutrino flux in the case of an additional sterile neutrino 224

9.11 Example of the sensitivity calculation to a 3+1 sterile neutrino hypothesis . . 225

9.12 The statistical-only sensitivity calculation to the MiniBooNE unfolded LEE signal . . . . . . . . . . . . . . . . . . . . . 226

10.1 MicroBooNE total Protons on Target collected with the Booster Neutrino Beam229 
A.1 The nonfunctional regions in the $Y Z$ projection requiring three active wire planes . . . . . . . . . . . . . . . . . . . . 232

A.2 Position dependent charge calibration factors in simulation. . . . . . . . . 233

B.1 Visualisation of the space charge effect using the start/end of cosmic muon

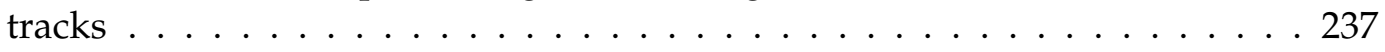

C.1 Energy dependence of cosmic muon track selection . . . . . . . . . . . . . . 242

C.2 Effect of simulated muon energy on the reconstructed zenith angle and track length . . . . . . . . . . . . . . . . . . . . . . 243

C.3 Cosmic muon flash creation efficiency for the geometrical position . . . . . 244

C.4 Flash creation efficiency for the muon track length and simulated energy . . . 244 
List of Figures 


\section{List of Tables}

2.1 $3 v$ oscillation parameters obtained from [13]. All five parameters are separately given with $1 \sigma$ intervals for both the case normal ordering or inverse ordering is assumed. . . . . . . . . . . . . . 14

2.2 Significance of experimental anomalies from neutrino experiments . . . . . 26

3.1 Neutrino flux at MicroBooNE from the BNB . . . . . . . . . . . . . . 38

5.1 Simulated integrated cosmic muon fluxes at MicroBooNE using different generators . . . . . . . . . . . . . . . . 77

5.2 Comparison of the measured and simulated cosmic muon rates in MicroBooNE102

6.1 Summary of the NeutrinoID passing rates for different samples of interest and different stages in the selection. The statistical error are $\mathcal{O}(0.1 \%) . \quad \ldots 129$

7.1 Event selection requirements for the $v_{\mu}$ CC selection . . . . . . . . . . . . 147

7.2 Muon identification requirements for the $v_{\mu}$ CC selection . . . . . . . . . . 152

8.1 Preselection requirements for the $v_{e}$ CC inclusive. . . . . . . . . . . . . 171

8.2 Input variables used for electron identification by the BDT. The data/MC distributions corresponding to the variables listed is given in Figure 8.16 . . . 181

8.3 Input variables used for other identification by the BDT. The data/MC distributions corresponding to the variables listed is given in Figures 8.19a and 8.19b.190

D.1 List of flux variations used in Chapter 9 to estimate the systematic uncertainties on the lepton kinematics. . . . . . . . . . . . . . . . . . . 246

D.2 List of GENIE systematic variations used used in Chapter 9 to estimate the systematic uncertainties on the lepton kinematics. . . . . . . . . . . . 247

D.3 Uncertainties included in All_Genie, see Table D.2 . . . . . . . . . . . . . . . 248 


\section{Bibliography}

[1] M. Thomson. Modern Particle Physics. Cambridge University Press, 2013. ISBN: 1107034264 .

[2] M. Ahmed et al. "Search for the lepton family number nonconserving decay $\mu \rightarrow$ $e+\gamma^{\prime \prime}$. In: Phys. Rev. D65 (2002), p. 112002. DOI: 10.1103/PhysRevD .65 . 112002. arXiv: hep-ex/0111030 [hep-ex].

[3] M. Aker et al. "Improved Upper Limit on the Neutrino Mass from a Direct Kinematic Method by KATRIN". In: Phys. Rev. Lett. 123.22 (2019), p. 221802. DOI: 10.1103 / PhysRevLett.123.221802. arXiv: 1909.06048 [hep-ex].

[4] Ettore Majorana. "Teoria simmetrica dell elettrone e del positrone. (Italian) [Symmetrical theory of the electron and the positron]". Italian. In: j-NUOVO-CIMENTO814.4 (Apr. 1937). In this paper, Majorana predicted the existence of a new type of particle, now called a Majorana fermion, which is its own antiparticle, and whose existence may have finally been confirmed by experiment seventy years later, pp. 171184. ISSN: 0029-6341 (print), 1827-6121 (electronic). DOI: https ://doi . org/10.1007/ BF02961314. URL: http:// en.wikipedia.org/wiki / Majorana_mass; \%20http: //nl.wikipedia.org/wiki/Majorana-deeltje.

[5] M. Goldhaber, L. Grodzins, and A. W. Sunyar. "Helicity of Neutrinos". In: Phys. Rev. 109 (3 Feb. 1958), pp. 1015-1017. DOI: 10.1103/PhysRev . 109 . 1015. URL: https: //Iink. aps.org/doi/10.1103/PhysRev.109.1015.

[6] Evgeny K. Akhmedov, G. C. Branco, and M. N. Rebelo. “Seesaw mechanism and structure of neutrino mass matrix". In: Phys. Lett. B478 (2000), pp. 215-223. DOI: 10. 1016/S0370-2693(00) 00282-3. arXiv: hep-ph/9911364 [hep-ph].

[7] M. Lindner, T. Ohlsson, and G. Seidl. "Seesaw mechanisms for Dirac and Majorana neutrino masses". In: Phys. Rev. D65 (2002), p. 053014.

[8] Yat-Long Chan et al. "Wave-packet treatment of reactor neutrino oscillation experiments and its implications on determining the neutrino mass hierarchy". In: The European Physical Journal C 76.6 (June 2016). ISSN: 1434-6052. DOI: 10.1140 / epjc / s10052-016-4143-4. URL: http://dx.doi.org/10.1140/epjc/s10052-016-4143-4.

[9] A. Yu. Smirnov. "The MSW effect and matter effects in neutrino oscillations". In: Phys. Scripta T121 (2005), pp. 57-64. DOI: 10 . 1088 / 0031 - 8949 / 2005/T121 / 008. arXiv: hep-ph/0412391 [hep-ph].

[10] C. Giunti and C. W. Kim. Fundamentals of Neutrino Physics and Astrophysics. Oxford University Press, 2007. ISBN: 0198508719. 
[11] P. Hernandez. "Neutrino physics". In: High-energy physics. Proceedings, 5th CERNLatin-American School, Recinto Quirama, Colombia, March 15-28, 2009. 2009. arXiv: 1010.4131 [hep-ph]. URL: http://inspirehep.net/record/873524/files/arXiv: 1010.4131.pdf.

[12] M. Tanabashi et al. "Review of Particle Physics". In: Phys. Rev. D 98 (3 Aug. 2018), p. 030001. DOI: 10.1103/PhysRevD.98.030001. URL: https://link. aps . org/doi/ 10.1103/PhysRevD.98.030001.

[13] P.F. de Salas et al. "Status of neutrino oscillations 2018: $3 \sigma$ hint for normal mass ordering and improved CP sensitivity". In: Physics Letters B 782 (July 2018), pp. 633640. ISSN: 0370-2693. DOI: 10.1016/j .physletb.2018.06.019. URL: http://dx. doi. org/10.1016/j.physletb.2018.06.019.

[14] Pablo F. de Salas et al. “Neutrino Mass Ordering from Oscillations and Beyond: 2018 Status and Future Prospects". In: Frontiers in Astronomy and Space Sciences 5 (2018), p. 36. ISSN: 2296-987X. DOI: 10 . 3389 / fspas . 2018 . 00036. URL: https : / / www . frontiersin.org/article/10.3389/fspas.2018.00036.

[15] S. Gariazzo et al. "Light sterile neutrinos". In: J. Phys. G43 (2016), p. 033001. DOI: 10.1088/0954-3899/43/3/033001. arXiv: 1507.08204 [hep-ph].

[16] G. H. Collin et al. "Sterile Neutrino Fits to Short Baseline Data". In: ArXiv e-prints (Feb. 2016). arXiv: 1602.00671 [hep-ph].

[17] A. Aguilar et al. "Evidence for neutrino oscillations from the observation of $\bar{v}_{e}$ appearance in a $\bar{v}_{\mu}$ beam". In: Phys. Rev. D 64 (11 Nov. 2001), p. 112007. DOI: $10.1103 /$ PhysRevD .64.112007. URL: http://Iink. aps .org/doi/10 .1103/PhysRevD .64. 112007.

[18] B. Armbruster et al. "Upper limits for neutrino oscillations $\bar{v}_{\mu} \rightarrow \bar{v}_{e}$ from muon decay at rest". In: Physical Review D 65.11 (June 2002). ISSN: 1089-4918. DOI: 10.1103/ physrevd.65.112001. URL: http://dx.doi.org/10.1103/PhysRevD .65.112001.

[19] James E. Hill. "An Alternative Analysis of the LSND Neutrino Oscillation Search Data on $\bar{v}_{\mu} \rightarrow \bar{v}_{e}{ }^{\prime \prime}$. In: Phys. Rev. Lett. 75 (14 Oct. 1995), pp. 2654-2657. DOI: 10.1103/ PhysRevLett. 75 . 2654. URL: https: // link.aps .org/doi/10 .1103/PhysRevLett. 75.2654.

[20] Boursin Louis et al. “A THOUSAND EYES : THE STORY OF LSND”. In: 1997.

[21] M Abbes et al. "The Bugey-3 neutrino detector". In: Nucl. Instrum. Meth. A 374 (1996), pp. 164-187. DOI: 10.1016/0168-9002 (96)00220-3.

[22] I. Stancu et al. "The MiniBooNE detector technical design report". In: (2001). DOI: 10.2172/809967.

[23] A. A. Aguilar-Arevalo et al. "Significant Excess of Electronlike Events in the MiniBooNE Short-Baseline Neutrino Experiment". In: Phys. Rev. Lett. 121 (22 Nov. 2018), p. 221801. DOI: 10.1103/PhysRevLett.121.221801. URL: https : / Iink . aps .org/ doi/10.1103/PhysRevLett.121.221801.

[24] A. A. Aguilar-Arevalo et al. "Measurement of $v_{\mu}$ and $\bar{v}_{\mu}$ induced neutral current single $\pi^{0}$ production cross sections on mineral oil at $E_{v} \sim \mathcal{O}(1 \mathrm{GeV})^{\prime \prime}$. In: Phys. Rev. D 81 (1 Jan. 2010), p. 013005. DOI: 10.1103/PhysRevD . 81.013005 . URL: https: //link.aps.org/doi/10.1103/PhysRevD.81.013005. 
[25] W. Hampel et al. "Final results of the 51Cr neutrino source experiments in GALLEX". In: Physics Letters B 420.1-2 (1998), pp. 114-126.

[26] J. N. Abdurashitov et al. "Measurement of the response of the Russian-American gallium experiment to neutrinos from a Cr-51 source". In: Phys. Rev. C59 (1999), pp. 2246-2263. DOI: 10.1103/PhysRevC. 59.2246. arXiv: hep-ph/9803418 [hep-ph].

[27] C. Giunti and M. Laveder. "Statistical Significance of the Gallium Anomaly". In: Phys. Rev. C83 (2011), p. 065504. DOI: 10.1103/PhysRevC . 83.065504. arXiv: 1006. 3244 [hep-ph].

[28] J. Kostensalo et al. "The gallium anomaly revisited". In: Physics Letters B 795 (Aug. 2019), pp. 542-547. ISSN: 0370-2693. DOI: 10 . 1016/ j . physletb . 2019 . 06 . 057. URL: http://dx.doi.org/10.1016/j.physletb.2019.06.057.

[29] A. Mueller et al. "Improved predictions of reactor antineutrino spectra". In: Phys. Rev. C 83 (5 May 2011), p. 054615. DOI: 10.1103/PhysRevC . 83.054615. URL: http: //Iink.aps.org/doi/10.1103/PhysRevC.83.054615.

[30] P. Huber. "Determination of antineutrino spectra from nuclear reactors". In: Phys. Rev. C 84 (2 Aug. 2011), p. 024617. DOI: 10.1103/PhysRevC. 84.024617. URL: http: //link.aps.org/doi/10.1103/PhysRevC.84.024617.

[31] J. Kopp et al. "Sterile neutrino oscillations: the global picture". In: Journal of High Energy Physics 2013.5 (2013), pp. 1-52.

[32] G. Mention et al. "Reactor antineutrino anomaly". In: Phys. Rev. D 83 (7 Apr. 2011), p. 073006. DOI: 10.1103/PhysRevD .83.073006. URL: http://link.aps .org/doi/10. 1103/PhysRevD . 83.073006.

[33] A. Gando et al. "White paper: CeLAND - Investigation of the reactor antineutrino anomaly with an intense ${ }^{144} \mathrm{Ce}-{ }^{144} \mathrm{Pr}$ antineutrino source in KamLAND". In: (2013). arXiv: 1309.6805 [hep-ex].

[34] M. Cribier. "Reactors antineutrino anomalies and searches for sterile neutrinos in Europe". In: Proceedings of the 15th International Workshop on Neutrino Telescopes. 2013.

[35] M. Archidiacono et al. "Sterile neutrinos: Cosmology versus short-baseline experiments". In: Phys. Rev. D87.12 (2013), p. 125034. DOI: 10 .1103/PhysRevD . 87.125034. arXiv: 1302.6720 [astro-ph.CO].

[36] R. A. Battye and A. Moss. "Evidence for Massive Neutrinos from Cosmic Microwave Background and Lensing Observations". In: Phys. Rev. Lett. 112 (5 Feb. 2014), p. 051303. DOI: 10.1103/PhysRevLett.112.051303. URL: http://Iink. aps . org/doi/10.1103/PhysRevLett.112.051303.

[37] C. Pena-Garay and A. Serenelli. "Solar neutrinos and the solar composition problem". In: (2008). arXiv: 0811.2424 [astro-ph].

[38] S. Gollapinni. "Accelerator-based Short-baseline Neutrino Oscillation Experiments". In: 12th Conference on the Intersections of Particle and Nuclear Physics. 2015. arXiv: 1510. 04412 [hep-ex]. URL: https://inspirehep. net/record/1398031/files/arXiv: 1510.04412.pdf.

[39] A. Diaz et al. "Where Are We With Light Sterile Neutrinos?" In: (2019). arXiv: 1906. 00045 [hep-ex]. 
[40] Artur M. Ankowski and Jan T. Sobczyk. "Argon spectral function and neutrino interactions". In: Phys. Rev. C74 (2006), p. 054316. DOI: 10.1103/PhysRevC . 74.054316. arXiv: nucl-th/0512004 [nucl-th].

[41] Lars Bathe-Peters. "Studies of Single Transverse Kinematic Variables for Neutrino Interactions on Argon". MA thesis. Harvard University, 2020.

[42] G. D. Megias, S. Dolan, and S. Bolognesi. "Modeling Neutrino-Nucleus Interactions for Neutrino Oscillation Experiments". In: Basic Concepts in Nuclear Physics: Theory, Experiments and Applications (2019), pp. 149-151. ISSN: 1867-4941. DOI: 10.1007/9783-030-22204-8_12. URL: http://dx.doi.org/10.1007/978-3-030-22204-8_12.

[43] A. A. Aguilar-Arevalo et al. "Neutrino flux prediction at MiniBooNE". In: Physical Review D 79.7 (Apr. 2009). ISSN: 1550-2368. DOI: 10.1103/physrevd. 79. 072002. URL: http://dx.doi.org/10.1103/PhysRevD.79.072002.

[44] The MicroBooNE Collaboration. "Booster Neutrino Flux Predition at MicroBooNE". In: MICROBOONE-NOTE-1031-PUB (2018). URL: http: / / microboone . fnal . gov / public-notes/.

[45] P. Adamson et al. "The NuMI neutrino beam". In: Nuclear Instruments and Methods in Physics Research Section A: Accelerators, Spectrometers, Detectors and Associated Equipment 806 (Jan. 2016), pp. 279-306. ISSN: 0168-9002. DOI: 10 . 1016/ j . nima. 2015.08. 063. URL: http://dx.doi.org/10.1016/j .nima.2015.08.063.

[46] Justin Evans. "The MINOS Experiment: Results and Prospects". In: Adv. High Energy Phys. 2013 (2013), p. 182537. DOI: 10.1155/2013/182537. arXiv: 1307. 0721 [hep-ex].

[47] Tomasz Golan, Leonidas Aliaga, and Mike Kordosky. "MINERvA's Flux Prediction". In: JPS Conf. Proc. 12 (2016), p. 010006. DOI: 10.7566/JPSCP. 12. 010006.

[48] R. Acciarri et al. "Demonstration of MeV-scale physics in liquid argon time projection chambers using ArgoNeuT". In: Phys. Rev. D 99 (1 Jan. 2019), p. 012002. DOI: 10 . 1103 / PhysRevD . 99 .012002. URL: https : / / link . aps .org/doi / 10 .1103/ PhysRevD . 99.012002.

[49] M. A. Acero et al. "First measurement of neutrino oscillation parameters using neutrinos and antineutrinos by NOvA". In: Phys. Rev. Lett. 123 (15 Oct. 2019), p. 151803. DOI: 10.1103/PhysRevLett.123.151803. URL: https://link. aps .org/doi/10 . 1103/PhysRevLett.123.151803.

[50] P. Adamson et al. "Search for Sterile Neutrinos in MINOS and MINOS+ Using a Two-Detector Fit". In: Phys. Rev. Lett. 122 (9 Mar. 2019), p. 091803. DOI: 10 . 1103 / PhysRevLett. 122.091803. URL: https : / / link . aps .org/doi/10.1103/ PhysRevLett.122.091803.

[51] G. Karagiorgi et al. "Viability of $\Delta m^{2} \sim 1 \mathrm{eV}^{2}$ sterile neutrino mixing models in light of MiniBooNE electron neutrino and antineutrino data from the Booster and NuMI beamlines". In: Phys. Rev. D 80 (7 Oct. 2009), p. 073001. DOI: 10.1103/PhysRevD . 80. 073001. URL: https://link. aps.org/doi/10.1103/PhysRevD . 80 .073001.

[52] MicroBooNE Collaboration. The MicroBooNE Technical Design Report. Tech. rep. 2012.

[53] The MicroBooNE Collaboration. "Design and construction of the MicroBooNE Cosmic Ray Tagger system". In: (2019). arXiv: 1901.02862 [physics.ins-det]. 
[54] Jay N. Marx and David R. Nygren. "The Time Projection Chamber". In: Phys. Today 31N10 (1978), pp. 46-53. DOI: 10.1063/1.2994775.

[55] C. Rubbia. "The Liquid Argon Time Projection Chamber: A New Concept for Neutrino Detectors". In: (1977).

[56] O. Bunemann, T. E. Cranshaw, and J. A. Harvey. “DESIGN OF GRID IONIZATION CHAMBERS". In: Canadian Journal of Research 27a.5 (1949), pp. 191-206. DOI: 10 . 1139/cjr49a-019. eprint: https : / / doi org/10.1139/cjr49a-019. URL: https: //doi.org/10.1139/cjr49a-019.

[57] R. Acciarri et al. "Noise Characterization and Filtering in the MicroBooNE Liquid Argon TPC". In: Journal of Instrumentation 12.08 (Aug. 2017), P08003-P08003. ISSN: 1748-0221. DOI: 10.1088/1748-0221/12/08/p08003. URL: http://dx. doi .org/10 . 1088/1748-0221/12/08/P08003.

[58] M. Del Tutto. "First measurements of inclusive muon neutrino charged current differential cross sections on argon at $0.8 \mathrm{GeV}$ average neutrino energy with the MicroBooNE detector". PhD thesis. University of Oxford, 2019.

[59] Robert S. Mulliken. "Potential Curves of Diatomic Rare-Gas Molecules and Their Ions, with Particular Reference to Xe2". In: The Journal of Chemical Physics 52.10 (1970), pp. 5170-5180. DOI: 10.1063/1.1672756. eprint: https://doi.org/10.1063/ 1.1672756. URL: https://doi.org/10.1063/1.1672756.

[60] T Katori. "The MicroBooNE light collection system". In: Journal of Instrumentation 8.10 (Oct. 2013), pp. C10011-C10011. ISSN: 1748-0221. DOI: 10 . 1088/1748-0221/8/ 10/c10011. URL: http://dx.doi.org/10.1088/1748-0221/8/10/C10011.

[61] Benjamin J. P. Jones. "Sterile Neutrinos in Cold Climates". PhD thesis. MIT, 2015. DOI: 10 . 2172 / 1221354. URL: http: / / Iss . fnal gov/archive/thesis / 2000 / fermilab-thesis-2015-17.pdf.

[62] V.M. Gehman et al. "Fluorescence efficiency and visible re-emission spectrum of tetraphenyl butadiene films at extreme ultraviolet wavelengths". In: Nuclear Instruments and Methods in Physics Research Section A: Accelerators, Spectrometers, Detectors and Associated Equipment 654.1 (2011), pp. 116-121. ISSN: 0168-9002. DOI: https : // doi.org/10.1016/j.nima.2011.06.088. URL: http://www.sciencedirect.com/ science/article/pii/S0168900211013271.

[63] The MicroBooNE Collaboration. "PMT Gain Calibration In MicroBooNE". In: MICROBOONE-NOTE-1064-TECH (2019). URL: https : / / microboone . fnal . gov / wp-content/uploads/MICROBOONE-NOTE-1064-TECH. pdf.

[64] D. Heck et al. "CORSIKA: A Monte Carlo Code to Simulate Extensive Air Showers". In: Forschungszentrum Karlsruhe Report FZKA (1998). URL: https : / /www . ikp . kit . edu/corsika/70.php.

[65] S. Agostinelli et al. In: Nucl. Instrum. Methods Phys. Res. 506.3 (2003), pp. 250-303.

[66] P. Abratenko et al. "Search for heavy neutral leptons decaying into muon-pion pairs in the MicroBooNE detector". In: Phys. Rev. D 101 (5 Mar. 2020), p. 052001. DOI: 10.1103/PhysRevD . 101.052001. URL: https : / / link . aps .org/doi/10 .1103/ PhysRevD .101.052001. 
[67] J I Crespo-Anadón. "The MicroBooNE continuous readout stream for detection of supernova neutrinos". In: Journal of Physics: Conference Series 1312 (Sept. 2019), p. 012006. ISSN: 1742-6596. DOI: 10 . 1088/1742-6596/1312/1/012006. URL: http: //dx.doi.org/10.1088/1742-6596/1312/1/012006.

[68] The MicroBooNE Collaboration. "Study of Reconstructed ${ }^{39} \mathrm{Ar}$ Beta Decays at the MicroBooNE Detector". In: MICROBOONE-NOTE-1050-PUB (2018). URL: http : / / microboone.fnal.gov/wp-content/uploads/MICROBOONE-NOTE-1050-PUB . pdf.

[69] Pedro A.N. Machado, Ornella Palamara, and David W. Schmitz. "The Short-Baseline Neutrino Program at Fermilab". In: Annual Review of Nuclear and Particle Science 69.1 (Oct. 2019), pp. 363-387. ISSN: 1545-4134. DOI: 10 . 1146 / annurev-nucl- 101917 020949. URL: http://dx.doi.org/10.1146/annurev-nucl-101917-020949.

[70] MicroBooNE Collaboration. "Design and Construction of the MicroBooNE Detector". In: JINST 12.P02017 (2017). URL: https: / / arxiv . org/abs/1612. 05824.

[71] C. Adams et al. "Ionization electron signal processing in single phase LArTPCs. Part II. Data/simulation comparison and performance in MicroBooNE". In: JINST 13.07 (2018), P07007. DOI: $10.1088 / 1748$ - 0221/13/07/P07007. arXiv: 1804.02583 [physics.ins-det].

[72] C. Adams et al. "Ionization electron signal processing in single phase LArTPCs. Part I. Algorithm Description and quantitative evaluation with MicroBooNE simulation". In: Journal of Instrumentation 13.07 (July 2018), P07006-P07006. ISSN: 1748-0221. DOI: 10 .1088/1748-0221/13/07/p07006. URL: http://dx.doi .org/10.1088/17480221/13/07/P07006.

[73] MicroBooNE Collaboration. "The Pandora multi-algorithm approach to automated pattern recognition of cosmic-ray muon and neutrino events in the MicroBooNE detector". In: Eur. Phys. J. C.78 (2018), p. 1. URL: https : / / arxiv . org/abs / 1708 . 03135.

[74] R Acciarri et al. "A study of electron recombination using highly ionizing particles in the ArgoNeuT Liquid Argon TPC". In: Journal of Instrumentation 8.08 (Aug. 2013), P08005-P08005. ISSN: 1748-0221. DOI: 10 . 1088 / 1748 - 0221 / 8 / 08 / p08005. URL: http://dx.doi.org/10.1088/1748-0221/8/08/P08005.

[75] MicroBooNE collaboration et al. Reconstruction and Measurement of $\mathcal{O}(100) \mathrm{MeV}$ Energy Electromagnetic Activity from $\pi^{0} \rightarrow \gamma \gamma$ Decays in the MicroBooNE LArTPC. 2019. arXiv: 1910.02166 [hep-ex].

[76] The MicroBooNE Collaboration. "Reconstruction Performance Studies with MicroBooNE Data". In: MICROBOONE-NOTE-1049-PUB (2018). URL: https : / / microboone. fnal gov/wp-content/uploads/MICROBOONE-NOTE-1049-PUB . pdf.

[77] The MicroBooNE Collaboration. "Cosmic Shielding Studies at MicroBooNE". In: MICROBOONE-NOTE-1005-PUB (2016). URL: http: / / microboone . fnal . gov/wpcontent/uploads/MICROBOONE-NOTE-1005-PUB . pdf.

[78] Chris Hagmann, David Lange, and Douglas Wright. “Cosmic-ray shower generator (CRY) for Monte Carlo transport codes". In: vol. 2. Jan. 2007, pp. 1143-1146. ISBN: 978-1-4244-0922-8. DOI: 10 .1109/NSSMIC . 2007 . 4437209. 
[79] C. Forti et al. "Simulation of atmospheric cascades and deep-underground muons". In: Phys. Rev. D 42 (11 Dec. 1990), pp. 3668-3689. DOI: 10 .1103/PhysRevD . 42 . 3668. URL: https://link. aps.org/doi/10.1103/PhysRevD.42.3668.

[80] S. Agostinelli et al. "GEANT4: A Simulation toolkit". In: Nucl. Instrum. Meth. A506 (2003), pp. 250-303. DOI: 10.1016/S0168-9002(03)01368-8.

[81] R. M. Sternheimer. "The Density Effect for the Ionization Loss in Various Materials". In: Phys. Rev. 88 (4 Nov. 1952), pp. 851-859. DOI: 10.1103/PhysRev . 88 . 851. URL: https://link.aps.org/doi/10.1103/PhysRev.88.851.

[82] The MicroBooNE Collaboration. "Measurement of cosmic-ray reconstruction efficiencies in the MicroBooNE LArTPC using a small external cosmic-ray counter". In: JINST 12.12 (2017), P12030. DOI: 10 . 1088/1748-0221/12/12/P12030. arXiv: 1707.09903 [hep-ex].

[83] Bogdan Mitrica et al. "A mobile detector for measurements of the atmospheric muon flux in underground sites". In: Nuclear Instruments and Methods in Physics Research Section A: Accelerators, Spectrometers, Detectors and Associated Equipment 654.1 (2011), pp. 176-183. ISSN: 0168-9002. DOI: https://doi .org/10.1016/ j.nima.2011.07.006. URL: http://www.sciencedirect.com/science/article/pii/S0168900211014082.

[84] D Stanca et al. "Measurements of the atmospheric muon flux using a mobile detector based on plastic scintillators read-out by optical fibers and PMTs". In: Journal of Physics Conference Series 409 (Feb. 2013), pp. 2136-. DOI: 10.1088/1742-6596/409/1/ 012136.

[85] S. Cecchini and M. Spurio. "Atmospheric muons: experimental aspects". In: Geoscientific Instrumentation, Methods and Data Systems 1 (Nov. 2012), pp. 185-196. DOI: 10.5194/gi-1-185-2012.

[86] The MicroBooNE Collaboration. "First Muon-Neutrino Charged-Current Inclusive Differential Cross Section Measurement for MicroBooNE Run 1 Data". In: MICROBOONE-NOTE-1045-PUB (2018). URL: http : / / microboone . fnal . gov/wpcontent/uploads/MICROBOONE-NOTE-1045-PUB . pdf.

[87] Polina Abratenko and MicroBooNE Collaboration. "Muon Momentum Determination with Multiple Coulomb Scattering for the MicroBooNE Experiment". In: APS April Meeting Abstracts. Vol. 2017. APS Meeting Abstracts. Jan. 2017, Y9.002.

[88] P. Abratenko et al. "First Measurement of Inclusive Muon Neutrino Charged Current Differential Cross Sections on Argon at $E_{v} \sim 0.8 \mathrm{GeV}$ with the MicroBooNE Detector". In: Phys. Rev. Lett. 123 (13 Sept. 2019), p. 131801. DOI: 10.1103/PhysRevLett. 123.131801. URL: https://link.aps.org/doi/10.1103/PhysRevLett.123.131801.

[89] C. Adams et al. "First measurement of $v_{\mu}$ charged-current $\pi^{0}$ production on argon with the MicroBooNE detector". In: Phys. Rev. D 99 (9 May 2019), p. 091102. DOI: 10. 1103 / PhysRevD . 99 .091102. URL: https : / / link . aps . org/doi / 10 . 1103/ PhysRevD . 99.091102.

[90] S. Dolan. "Exploring Nuclear Effects in Neutrino-Nucleus Interactions Using Measurements of Transverse Kinematic Imbalance from T2K and MINERvA". In: (2018). arXiv: 1810.06043 [hep-ex]. 
[91] C. Adams et al. "Calibration of the charge and energy loss per unit length of the MicroBooNE liquid argon time projection chamber using muons and protons". In: (2019). arXiv: 1907.11736 [physics.ins-det].

[92] Glen Cowan et al. "Asymptotic formulae for likelihood-based tests of new physics (vol 71, pg 1554, 2011)”. In: European Physical Journal C 73 (July 2013). DOI: 10.1140/ epjc/s10052-013-2501-z.

[93] The MicroBooNE Collaboration. "The MicroBooNE Search for Single Photon Events". In: MICROBOONE-NOTE-1041-PUB (2018). URL: http : / / microboone . fnal . gov / wp-content/uploads/MICROBOONE-NOTE-1041-PUB.pdf.

[94] The MicroBooNE Collaboration. "MicroBooNE low-energy excess signal prediction from unfolding MiniBooNE Monte-Carlo and data". In: MICROBOONE-NOTE1043-PUB (2018). URL: http : / / microboone. fnal . gov / wp - content / uploads / MICROBOONE-NOTE-1043-PUB.pdf.

[95] G. D'Agostini. "A Multidimensional unfolding method based on Bayes' theorem". In: Nucl. Instrum. Meth. A362 (1995), pp. 487-498. DOI: 10 . 1016/0168-9002(95) 00274-X.

[96] R. Acciarri et al. "First observation of low energy electron neutrinos in a liquid argon time projection chamber". In: Phys. Rev. D 95 (7 Apr. 2017), p. 072005. DOI: 10.1103/ PhysRevD . 95 .072005. URL: https : / / link. aps .org/doi/10 .1103/PhysRevD . 95. 072005.

[97] H. A. Bethe. "Molière's Theory of Multiple Scattering". In: Phys. Rev. 89 (6 Mar. 1953), pp. 1256-1266. DOI: 10.1103/PhysRev.89.1256. URL: https://link.aps.org/doi/ 10.1103/PhysRev. 89.1256.

[98] C. Adams. "First Detection of Low Energy Electron Neutrinos in Liquid Argon Time Projection Chambers". PhD thesis. Yale University, 2017. URL: https : // Iss . fnal. gov/archive/thesis/2000/fermilab-thesis-2016-42.pdf.

[99] D. Caratelli. "Study of Electromagnetic Interactions in the MicroBooNE Liquid Argon Time Projection Chamber". PhD thesis. Columbia University, 2018. URL: http: //inspirehep.net/record/1654724.

[100] Tianqi Chen and Carlos Guestrin. "XGBoost: A Scalable Tree Boosting System". In: Proceedings of the 22nd ACM SIGKDD International Conference on Knowledge Discovery and Data Mining. KDD '16. San Francisco, California, USA: ACM, 2016, pp. 785-794. ISBN: 978-1-4503-4232-2. DOI: 10 . 1145/2939672 . 2939785. URL: http : / / doi . acm . org/10.1145/2939672.2939785.

[101] Jerome H. Friedman. "Greedy Function Approximation: A Gradient Boosting Machine". In: The Annals of Statistics 29.5 (2001), pp. 1189-1232. ISSN: 00905364. URL: http://www.jstor.org/stable/2699986.

[102] Brad Boehmke. Hands-on machine learning with R. Boca Raton, FL: CRC Press, 2020. ISBN: 9781138495685.

[103] D. Cianci et al. "Prospects of light sterile neutrino oscillation and CP violation searches at the Fermilab Short Baseline Neutrino Facility". In: Physical Review D 96.5 (Sept. 2017). ISSN: 2470-0029. DOI: 10 .1103/physrevd. 96.055001 . URL: http: //dx.doi.org/10.1103/PhysRevD.96.055001. 
[104] The MicroBooNE Collaboration. "Study of Space Charge Effects in MicroBooNE". In: MICROBOONE-NOTE-1018-PUB (2016). URL: http : / / microboone f fnal . gov / wp-content/uploads/MICROBOONE-NOTE-1018-PUB.pdf.

[105] E Catano-Mur and. "Electron neutrino appearance in the NOvA experiment". In: Journal of Physics: Conference Series 888 (Sept. 2017), p. 012062. DOI: 10.1088/17426596/888/1/012062. URL: https : // doi . org/10 . 1088\%2F1742-6596\%2F888\%2F1\% $2 \mathrm{~F} 012062$.

[106] Adam Lister. "Constraint of systematic uncertainties in an electron neutrino search using muon neutrinos at MicroBooNE". English. PhD thesis. Lancaster University, 2019. DOI: 10.17635/lancaster/thesis/632.

[107] M. H. Shaevitz. Constraining the $v_{e}$ Background Uncertainties with the Observed $v_{\mu}$ Events. Tech. rep. Columbia University, 2007.

[108] G. Cowan. Statistical Data Analysis. Oxford science publications. Clarendon Press, 1998. ISBN: 9780198501558. URL: https://books . google.com/books? id=ff8ZyWOnlJAC.

[109] Mona Dentler et al. "Updated Global Analysis of Neutrino Oscillations in the Presence of eV-Scale Sterile Neutrinos". In: JHEP 08 (2018), p. 010. DOI: 10.1007 / JHEP08 (2018) 010. arXiv: 1803.10661 [hep-ph] .

[110] Spencer Nicholas Gaelan Axani. "Sterile Neutrino Searches at the IceCube Neutrino Observatory". PhD thesis. MIT, 2019. arXiv: 2003.02796 [hep-ex].

[111] A.P. Serebrov et al. "First Observation of the Oscillation Effect in the Neutrino-4 Experiment on the Search for the Sterile Neutrino". In: Pisma Zh. Eksp. Teor. Fiz. 109.4 (2019), pp. 209-218. DOI: 10.1134/S0021364019040040. arXiv: 1809.10561 [hep-ex].

[112] Mikhail Danilov. "Recent results of the DANSS experiment". In: 2019 European Physical Society Conference on High Energy Physics. Nov. 2019. arXiv: 1911.10140 [hep-ex] .

[113] S. R. Soleti. "Search for a low-energy excess of electron neutrinos in MicroBooNE". PhD thesis. University of Oxford, 2019. 